\title{
Optische Eigenschaften von verdünnten magnetischen Halbleitern auf GaN-Basis
}

\author{
Dissertation \\ zur Erlangung des Doktorgrades \\ der Mathematisch-Naturwissenschaftlichen Fakultäten \\ der Georg-August-Universität zu Göttingen
}

vorgelegt von

Jan Zenneck

aus Marburg

Göttingen, 2007 
D7

Referent: Frau Prof. Dr. A. Rizzi

Korreferent: Herr Prof. Dr. H. Hofsäss

Tag der mündlichen Prüfung: 18.07.2007 


\section{Inhaltsverzeichnis}

1 Einleitung 1

2 Physikalische Grundlagen 3

2.1 Physikalische Eigenschaften von GaN . . . . . . . . . . . . . . . 3

2.1.1 Strukturelle Eigenschaften . . . . . . . . . . . . . . 4

2.1 .2 Verspannungen in GaN . . . . . . . . . . . . . . . . 5

2.1.3 Elektronische Eigenschaften _. . . . . . . . . . . . 6

2.2 Kristallfeldtheorie . . . . . . . . . . . . . . . . . . . . . . . 9 9

2.2.1 Übergangsmetalle in tetraedrischer und oktaedrischer Umgebung . . 9

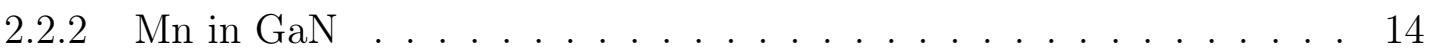

2.2 .3 Optische Übergänge und Magneto-Optik . . . . . . . . . . . . . . 19

2.3 Verdünnte magnetische Halbleiter (DMS) . . . . . . . . . . . . . . 23

2.3 .1 Magnetische Kopplung . . . . . . . . . . . . . . . . . . . . . . . . 23

2.3 .2 DMS auf GaN-Basis . . . . . . . . . . . . . . . 27

3 Wachstum von GaN und GaMnN 31

3.1 Molekularstrahl-Epitaxie (MBE) . . . . . . . . . . . . . . . . 31

3.2 Parameter-Raum des MBE-Wachstums von GaN . . . . . . . . . . 32

$3.3 \mathrm{GaN}$ auf verschiedenen Substraten . . . . . . . . . . . . . . . . 35

4 Experimentelle Methoden 37

4.1 Photolumineszenz $(\mathrm{PL}) \ldots \ldots \ldots$

4.1 .1 Lumineszenz in Halbleitern _. . . . . . . . . . . . . . 37

4.1 .2 Exzitonen . . . . . . . . . . . . . . . . . . . 40

4.1 .3 Lumineszenz von $\mathrm{GaN} \ldots \ldots$. . . . . . . . . . . . . . . . 41

4.1 .4 Experimenteller Aufbau . . . . . . . . . . . . . . . . . . 44

4.2 Raman-Streuung . . . . . . . . . . . . . . . . . . . . . . 47

4.3 Weitere Messmethoden . . . . . . . . . . . . . . . . . . . 53

4.3 .1 Absorption . . . . . . . . . . . . . . . . 53

4.3.2 Rutherford-Rückstreuung (RBS) und Implantation . . . . . . . . 53

4.3.3 Sekundär-Ionen Massenspektroskopie (SIMS) . . . . . . . . . 55

4.3.4 Röntgen-Diffraktometrie (XRD) . . . . . . . . . . . . . . 57 
5 Struktur und Phasen bei verdünnten GaMnN-Schichten 59

5.1 Bildung einer zweiten Phase . . . . . . . . . . . . . . . . . . . . 59

5.1 .1 Nachweis $\operatorname{im}$ XRD . . . . . . . . . . . . . . . . . . . . 59

5.1 .2 Identifikation im TEM . . . . . . . . . . . . . . . . . . 63

5.1 .3 Wachstum ohne Ausscheidungen . . . . . . . . . . . . . . . 64

5.2 Strukturelle Eigenschaften von GaMnN . . . . . . . . . . . . . . . . 67

5.2 .1 Veränderung mit der Wachstumstemperatur . . . . . . . . . . 67

5.2 .2 Einfluss von Ga- und Mn-Angebot . . . . . . . . . . . . . . . 69

5.2 .3 Einfluss des Substrates . . . . . . . . . . . . . . . . 72

5.3 RBS-Messungen und Gitterplatzbestimmung von Mn . . . . . . . . 74

6 Optische Eigenschaften von GaMnN 77

6.1 PL vom UV bis sichtbaren Bereich . . . . . . . . . . . . . . . . . . 77

6.1.1 Abhängigkeit von den Wachstumsparametern _. . . . . . . . . 79

6.1 .2 Abhängigkeit vom Substrattyp . . . . . . . . . . . . . . . . . 82

6.1.3 Kompensierende Wirkung von $\mathrm{Mn}$ in GaMnN . . . . . . . . . . . . 84

6.2 PL im Infraroten . . . . . . . . . . . . . . . . . . . . . . . 87

6.2.1 Identifikation des optischen Übergangs bei $1.4 \mathrm{eV} \ldots \ldots$. . . . . 87

6.2.2 Abhängigkeit von den Wachstumsparametern . . . . . . . . . . . 91

6.2 .3 Verbesserung durch Saphir-Substrat . . . . . . . . . . . . . . . 93

6.2 .4 Anregungsmechanismus . . . . . . . . . . . . . . . . . . . . . . . 99

6.3 Magneto-optische Messungen _. . . . . . . . . . . . . . . . . . . . 101

6.3.1 Nahe der Bandkante . . . . . . . . . . . . . . . . . . . . . 101

$6.3 .2 \quad$ Bei $1.4 \mathrm{eV} \ldots \ldots \ldots \ldots \ldots \ldots$

7 Weitere GaN-basierte DMS 113

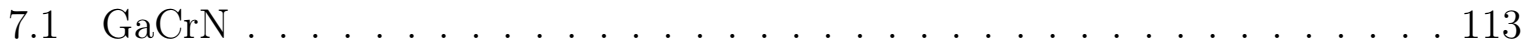

7.1.1 Wachstumsbedingungen . . . . . . . . . . . . . 113

7.1.2 PL vom UV bis sichtbaren Bereich . . . . . . . . . . . . . . . 114

7.1 .3 PL im Infraroten . . . . . . . . . . . . . . . . . . . . . . . 119

7.2 GaGdN . . . . . . . . . . . . . . . . . . . . . . . . . . . . 122

7.2 .1 Wachstumsbedingungen . . . . . . . . . . . . . . 122

7.2 .2 Bandkantennahe PL . . . . . . . . . . . . . . . . . . . . . . . 122

8 Zusammenfassung und Ausblick 127

Anhang A: Korrektur der temperaturabhängigen Messungen 130

Anhang B: Überblick über die GaMnN-Proben 132

$\begin{array}{ll}\text { Abbildungsverzeichnis } & 133\end{array}$

$\begin{array}{ll}\text { Tabellenverzeichnis } & 137\end{array}$

$\begin{array}{ll}\text { Literaturverzeichnis } & 138\end{array}$ 


\section{Verzeichnis der Abkürzungen}

\begin{tabular}{|c|c|}
\hline $\mathrm{ABX}$ & Akzeptor-gebundenes Exziton (acceptor bound exciton) \\
\hline AFM & Rasterkraftmikroskopie (atomic force microscopy) \\
\hline ALCHEMI & (atom localisation by channeling enhanced microanalysis) \\
\hline AlN & Aluminium-Nitrid \\
\hline BEP & (beam equivalent pressure) \\
\hline BX & gebundenes Exziton \\
\hline CL & Kathodolumineszenz (cathodoluminescence) \\
\hline DARS & $\begin{array}{l}\text { Unordnungs-aktivierte Raman-Streuung (disorder-activated Raman scatte- } \\
\text { ring) }\end{array}$ \\
\hline DAP & Donator-Akzeptor-Paar-Übergang \\
\hline DBX & Donator-gebundenes Exziton (donor bound exciton) \\
\hline DFT & Dichtefunktional-Theorie (density functional theory) \\
\hline DMS & verdünnter magnetischer Halbleiter (diluted magnetic semiconductor) \\
\hline DRS & (direct recoil spectroscopy) \\
\hline EDX & energiedispersive Röntgenspektroskopie (energy dispersive x-ray) \\
\hline EXAFS & (extended x-ray absorption fine structure) \\
\hline FWHM & Halbwertsbreite (full width at half maximum) \\
\hline $\mathrm{GaN}$ & Gallium-Nitrid \\
\hline GL & grüne Lumineszenz von GaN \\
\hline HVPE & Hydrid-Gasphasen-Epitaxie (hydride vapor phase epitaxy) \\
\hline $\operatorname{InN}$ & Indium-Nitrid \\
\hline IR & Infrarot \\
\hline $\mathrm{LO}$ & longitudinal-optische Mode \\
\hline LVM & lokale Schwingungsmode (local vibrational mode) \\
\hline MBE & Molekularstrahl-Epitaxie (molecular beam epitaxy) \\
\hline MOCVD & $\begin{array}{l}\text { Metall-organische Gasphasen-Epitaxie (metal-organic chemical vapor depo- } \\
\text { sition) }\end{array}$ \\
\hline PL & Photolumineszenz \\
\hline RBS & Rutherford-Rückstreuung (Rutherford backscattering) \\
\hline $\mathrm{RBS} / \mathrm{C}$ & RBS channeling \\
\hline RL & rote Lumineszenz von GaN \\
\hline SEM & Raster-Elektronenmikroskopie (scanning electron microscopy) \\
\hline SIMS & Sekundärionen-Massenspektroskopie \\
\hline TEM & Transmissions-Elektronenmikroskopie \\
\hline $\mathrm{TO}$ & transversal-optische Mode \\
\hline TOF & Flugzeit (time of flight) \\
\hline UV & Ultraviolett \\
\hline $\mathrm{XRD}$ & Röntgenbeugung (x-ray diffraction) \\
\hline YL & gelbe Lumineszenz von GaN (yellow luminescence) \\
\hline ZPL & Null-Phononen-Linie (zero phonon line) \\
\hline
\end{tabular}





\section{Einleitung}

Die Nitride im Allgemeinen und Gallium-Nitrid (GaN) im Speziellen haben im Laufe der letzten Jahre große Forschungstätigkeit erfahren und sich in immer mehr Anwendungen durchgesetzt. In der Optoelektronik waren die ersten blauen LEDs (light emitting diodes) auf GaN-Basis eine Revolution, da sie im Vergleich zu den bis dato konkurrenzlosen SiCbasierten LEDs eine um Größenordnungen höhere Quanteneffizienz besaßen. Obwohl die entsprechenden Laborresultate erst 1994 präsentiert wurden [1], werden sie mittlerweile für viele Anwendungen eingesetzt. So bilden sie beispielsweise die Basis für die weißen LEDs, die gerade im Begriff sind, sämtliche Bereiche der Beleuchtungstechnik zu erobern. Durch Einlegieren verschieden großer Indium-Anteile sind auch violette [2], grüne [3] und gelbe [4] LEDs konstruiert worden. Der nächste Schritt, die Konstruktion einer blauen Laserdiode, gelang Nakamura et al. schließlich 1996 [5].

Auch die besonderen elektronischen Eigenschaften von GaN werden beispielsweise für Transistoren mit hoher Leistung und hoher Frequenz ausgenutzt. Ansonsten ist für fast alle elektronischen Bauteile nach wie vor die Silizium-Technologie Standard.

Eine ganz neue Art von Elektronik ergibt sich bei Nutzung des Spins der Ladungsträger in den Bauteilen. Man spricht dann von so genannter Spin-Elektronik oder kurz Spintronik. Dieses Gebiet ist bisher hauptsächlich theoretisch ergründet, so schlugen z. B. Datta und Das einen Spin-Transistor vor [6], bei dem magnetische Kontakte spinpolarisierte Ströme injizieren bzw. detektieren, während im dazwischen liegenden Kanal eine Manipulation der Spinrichtung über den Rashba-Effekt durch eine Gate-Elektrode erfolgt. Eine große Herausforderung liegt dabei in der Konstruktion der magnetischen Elektroden, da klassische (metallische) Ferromagneten durch den großen Leitfähigkeitsunterschied keine effektive Spininjektion zeigen [7]. Als Alternative wurden daher verdünnte magnetische Halbleiter (DMS) ins Spiel gebracht. Im Laufe der 90er Jahre des letzten Jahrhunderts konnten durch Fortschritte bei der Präparation der III-V-Halbleiter tatsächlich magnetische Halbleiter auf Basis von GaMnAs realisiert werden. Allerdings waren die erreichbaren Curie-Temperaturen dabei auf den Bereich bis etwa $100 \mathrm{~K}$ begrenzt. Durch Verbesserung des Materials und der Nachbehandlung ist inzwischen die maximal erreichte Curie Temperatur von GaMnAs auf $170 \mathrm{~K}$ gestiegen (siehe z. B. [8] und dortige Referenzen).

Im Jahr 2000 schließlich veröffentlichten Dietl et al. [9] ein theoretisches Modell, nach dem unter bestimmten Vorraussetzungen GaMnN eine $\mathrm{T}_{C}$ oberhalb Raumtemperatur aufweist. Der wesentliche Unterschied zum GaMnAs ist im Rahmen dieses Modells die kleinere Gitterkonstante, welche eine stärkere Wechselwirkung zwischen den Mn-Atomen erlaubt. Diese Theorie regte vielfältige experimentelle und theoretische Arbeiten an. Auf experimenteller Seite wurde GaMnN mal als Antiferromagnet [10], Paramagnet [11] oder Ferromagnet mit einer Curie-Temperatur bis $940^{\circ} \mathrm{C}$ [12] identifiziert, während verschiedene weiterführende Theorien $\mathrm{T}_{C}$ mal oberhalb [13] und mal weit unterhalb Raumtempera- 
tur [14] vorhersagten. Auch andere Übergangsmetalle wie Chrom und Vanadium wurden als Kandidaten für einen DMS bei Raumtemperatur ins Spiel gebracht [13], ebenso Seltene Erden wie Gadolinium [15].

Zum aktuellen Zeitpunkt ist weder der Mechanismus der magnetischen Kopplung noch die prinzipielle Eignung von GaMnN oder anderer GaN-basierter DMS bei Raumtemperatur geklärt. Eine Verbesserung dieses Zustands kann nur erreicht werden, wenn die elektronische Struktur von Mn in GaN genau bekannt ist. Einen direkten Zugang dazu bieten optische Messungen, da auf Grund der vielen optischen Anwendungen das System GaN relativ gut untersucht ist. Beispielsweise konnte mittels Absorption ein intra3d-Übergang von $\mathrm{Mn}^{3+}$ beobachtet werden [16], der gleichsam als Fingerabdruck diesen Ladungszustand in den entsprechenden GaMnN-Proben anzeigt. Andererseits können magneto-optische Messungen an den bandkantennahen Exzitonen direkt eine Kopplung der GaN-Ladungsträger mit den Mn-Spins anzeigen [17]. Noch ist es allerdings nicht gelungen (bis auf einen Fall bei hohen Magnetfeldern [18]), mittels einer p-i-n-Diode in diesem System polarisierte Lichtemission und damit Spininjektion nachzuweisen [19]. Dies wäre der finale Machbarkeitsbeweis für GaMnN als Basismaterial der Spintronik.

Um diesem Ziel näher zu kommen, sollen im Rahmen dieser Arbeit die optischen Eigenschaften von GaN-basierten DMS untersucht werden, mit Schwerpunkt auf GaMnN. Nur ein vollständiges Verständnis der Auswirkungen des Mn-Einbaus auf die Lumineszenz von GaN ermöglicht die volle Anwendbarkeit dieser Methode zur Charakterisierung verdünnter magnetischer Halbleiter. Es müssen dabei vor allem Effekte getrennt werden, die durch die unterschiedlichen Wachstumsbedingungen einerseits und die unterschiedlichen Mn-Konzentrationen andererseits verursacht werden.

Die Arbeit gliedert sich daher wie folgt: In Kapitel 2 werden die grundlegenden physikalischen Sachverhalte geklärt. Dies beinhaltet die strukturellen und elektronischen Eigenschaften von GaN, die Grundlagen der Kristallfeldtheorie, der intra-3d-Übergänge und ein Überblick über den Stand der Forschung auf dem Gebiet der GaN-basierten DMS. Kapitel 3 beschreibt das epitaktische Wachstum von GaN und die wichtigsten Parameter, die Einfluss auf das Wachstum haben. Die im Rahmen dieser Arbeit verwendeten experimentellen Methoden sind in Kapitel 4 beschrieben. Das wichtigste Werkzeug war dabei die Photolumineszenz, es kamen aber auch andere, vor allem strukturelle, Charakterisierungsmethoden zum Einsatz. Die Ergebnisse dieser strukturellen Charakterisierung sind in Kapitel 5 dargelegt. An erster Stelle steht dabei das Verhindern von Ausscheidungen beim Wachstum und die Definition von Wachstumsbedingungen, bei denen möglichst hochqualitatives GaMnN gewachsen werden kann. Auf Basis dieser Ergebnisse werden in Kapitel 6 die optischen Eigenschaften von GaMnN untersucht. Speziell die exzitonische Lumineszenz und ein intra-3d-Übergang im Infraroten stehen dabei im Vordergrund. Deren Eigenschaften werden umfassend charakterisiert. Zusätzlich werden dabei magneto-optische Experimente durchgeführt. In Kapitel 7 schließlich werden mit $\mathrm{GaCrN}$ und GaGdN zwei weitere Kandidaten für Ferromagnetismus bei Raumtemperatur untersucht. GaCrN zeigt dabei einige Ähnlichkeiten zu den GaMnN-Proben, während in GaGdN zwar keine internen Übergänge detektierbar sind, dafür aber Gitterverspannungen eine große Rolle spielen. 


\section{Physikalische Grundlagen}

In diesem Kapitel sollen grundlegende physikalische Gegebenheiten erörtert werden, die für die durchgeführten experimentellen Untersuchungen von Belang sind. Dies bezieht sich auf die Materialeigenschaften von GaN, dem Halbleiter, mit dem sich diese Arbeit beschäftigt und seine Eignung als verdünnter magnetischer Halbleiter. Die den magnetischen Halbleitern zu Grunde liegende Theorie soll ebenso kurz geklärt werden wie die Auswirkungen des so genannten Kristallfeldes auf die elektronischen Zustände von Übergangsmetallen.

\subsection{Physikalische Eigenschaften von GaN}

\begin{tabular}{|c||c|l|}
\hline Kenngröße & Wert & Kommentar \\
\hline Atomdichte $\left[\mathrm{cm}^{-3}\right]$ & $8.8 \times 10^{22}$ & \\
Dichte $\rho\left[\mathrm{g} / \mathrm{cm}^{3}\right]$ & 6.15 & \\
Gitterkonstante a $[\AA]$ & 3.189 & \\
Gitterkonstante c $[\AA]$ & 5.186 & \\
Bandlücke $E_{g}[\mathrm{eV}]$ & 3.44 & aus PL-Messungen \\
Statische Dielektrizitätskonstante & 8.9 & \\
Effektive Elektronenmasse $\left[\mathrm{m}_{0}\right]$ & 0.2 & \\
Effektive Lochmasse $\left[\mathrm{m}_{0}\right]$ & $1.0-1.6$ & z. T. widersprüchliche Angaben \\
Brechungsindex (infrarot) & 2.3 & \\
Schmelztemperatur $[\mathrm{K}]$ & 2791 & bei $60 \mathrm{kbar}$ \\
Wärmeleitfähigkeit $[W / \mathrm{cm} \cdot \mathrm{K}]$ & 1.3 & \\
Durchbruchfeldstärke $[\mathrm{V} / \mathrm{cm}]$ & $3.3 \times 10^{6}$ & \\
Sättigungsgeschwindigkeit $[\mathrm{m} / \mathrm{s}]$ & $2.7 \times 10^{5}$ & \\
\hline
\end{tabular}

Tabelle 2.1: Wichtige physikalische Kenngrößen von GaN bei $300 K$ (siehe $[20,21,22]$ und dortige Referenzen)

GaN weist einige besondere Eigenschaften auf, die es für bestimmte Anwendungen sehr interessant machen. Wichtige physikalische Größen sind in Tabelle 2.1 zusammengefasst. Seine große Bandlücke beispielsweise ermöglicht es, durch Legieren optoelektronische Bauelemente im gesamten sichtbaren Spektralbereich zu konstruieren. So wurden mit GaN die ersten weißen LEDs hergestellt [23], die mittlerweile auch kommerziell überall erhältlich sind. Außerdem gehört GaN zu den superharten Materialien, was seine Handhabung sehr einfach macht. Auch chemisch und thermisch ist es sehr stabil, erst bei hohen Temperaturen (über $800^{\circ} \mathrm{C}$ ) zersetzt es sich. Seine thermische Leitfähigkeit ist sehr gut und vor 
allem die hohe Durchbruchfeldstärke und hohe Sättigungsgeschwindigkeit macht es sehr geeignet für Einsätze im Bereich hoher Leistungen und hoher Frequenz.

\subsubsection{Strukturelle Eigenschaften}

Wie die meisten Gruppe-III-Nitride kann auch GaN im Wesentlichen in zwei unterschiedlichen Kristallstrukturen existieren, der (metastabilen) kubischen Zinkblende-Struktur oder der hexagonalen Wurtzit-Struktur. In beiden Fällen ist das Kation (Gruppe III) von jeweils vier Anionen (Gruppe V) tetraedrisch umgeben und umgekehrt. Die tetraedrische Anordnung ist Folge der chemischen Bindung, die von $\mathrm{sp}^{3}$-Hybridorbitalen gebildet wird. Die beiden Kristalltypen unterscheiden sich nur in der Schichtfolge, die bei der hexagonalen Phase ABABAB, bei der kubischen aber ABCABC ist, wie in Abbildung 2.1 gezeigt. Bei GaN ist die Wurtzit-Struktur die thermodynamisch stabile Phase, allerdings kann wegen des geringen Energieunterschiedes auch die Zinkblende-Struktur während des Wachstums beispielsweise durch geeignete Substrate stabilisiert werden. Unter extrem hohen Drücken existiert schließlich eine Phase in Kochsalz-Struktur. Die im Rahmen dieser Arbeit untersuchten Proben liegen alle in der Wurtzit-Struktur vor.

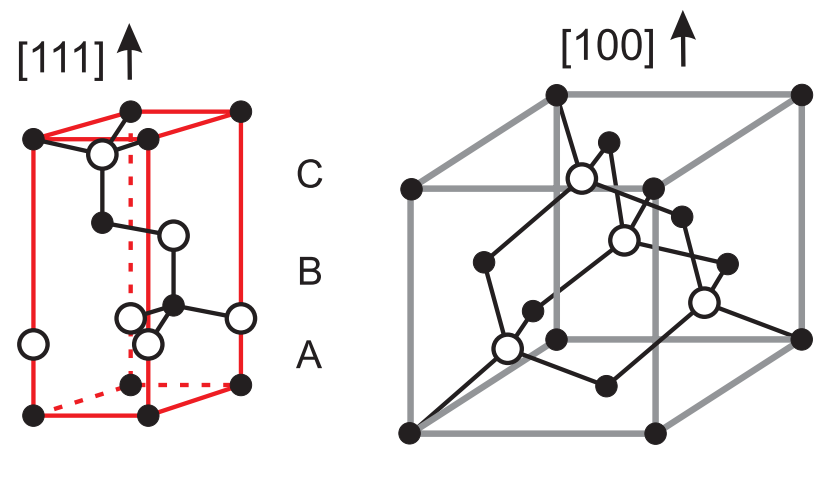

Zinkblende

O N

- $\mathrm{Ga}$

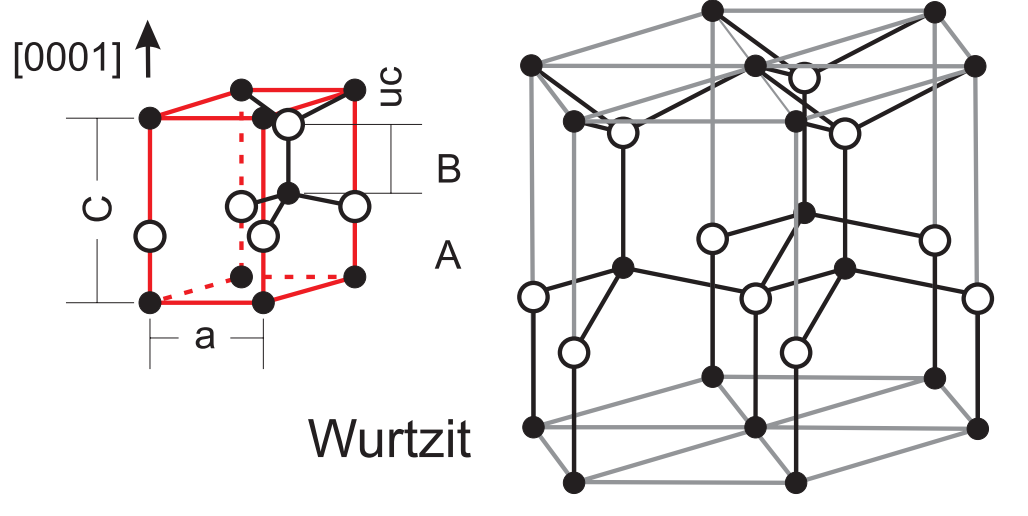

Wurtzit

[0001] $\uparrow$

Abbildung 2.1: Zinkblende und Wurtzit: Kristallstruktur.

In Wurtzit-Kristallen sind die [0001]- und [0001]]-Richtungen nicht äquivalent, wie man 
in Abbildung 2.1 erkennen kann. Jede „Monolage“ besteht in Wirklichkeit aus einem GaN-Dimer, die aufeinander folgenden Ga- und N-Schichten besitzen aber verschiedene Abstände. Die (0001)-Ebene (oben liegend in Abbildung 2.1) wird dabei als Ga-polar bezeichnet, (0001) als N-polar. Diese Bezeichnung muss klar unterschieden werden von Gabzw. N-terminierten Flächen, was sich auf die tatsächlichen endständigen Atome bezieht.

Ein wesentlicher Unterschied zwischen den beiden Kristalltypen ist die im Wurtzit vorhandene spontane Polarisation. Sowohl im kubischen als auch im hexagonalen Fall kann man sich den Kristall aus Atomebenen ((111) für Zinkblende, (0001) für Wurtzit) jeweils einer Sorte aufgebaut vorstellen. Auf Grund der teilweise ionischen Natur der Bindung zwischen Gallium und Stickstoff gibt es relativ starke Dipolmomente, die sich zwar im Volumen aufheben (sofern der Kristall unverspannt ist), an den Grenzflächen aber zu Polarisationsladungen führen. In der kubischen Phase existieren vier äquivalente Rich-

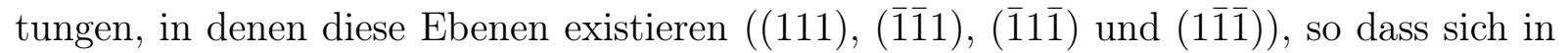
der Addition keine spontane Polarisation ergibt. In der hexagonalen Phase mit ihrer niedrigeren Symmetrie fehlen weitere polare Richtungen und die Polarisation in c-Richtung tritt schon im unverspannten Kristall spontan auf. Die experimentelle Bestimmung der spontanen Polarisation ist schwierig, da sie durch andere Effekte überlagert wird. Allerdings existieren ab initio-Berechnungen von Bernardini et al. [24], die einen Wert von $0.029 \mathrm{C} / \mathrm{m}^{2}$ für GaN ergaben.

Wird ein Kristall verspannt, wie es zum Beispiel beim Wachstum auf einem Substrat mit anderer Gitterkonstante der Fall ist (siehe Kapitel 3.3), so wird die Symmetrie erniedrigt. Die Polarisationen, die sich im kubischen Zinkblendekristall zu Null addierten, gleichen sich nun nicht mehr exakt aus und es kommt zu einer so genannten ,piezoelektrischen Polarisation“. Der gleiche Effekt tritt auch bei Wurtzit-Kristallen zusätzlich zur spontanen Polarisation auf. Die Gesamtpolarisation in GaN berechnet sich also nach

$$
P_{g e s}=P_{s p}+P_{p z}
$$

wobei $P_{s p}$ die spontane Polarisation bezeichnet und $P_{p z}$ die piezoelektrische, die verschiedene Vorzeichen haben kann, je nach Art der Verspannung.

\subsubsection{Verspannungen in GaN}

Es ist extrem schwierig und aufwändig, GaN als Volumenmaterial herzustellen, daher wird es normalerweise mittels Epitaxie auf Heterosubstraten hergestellt (siehe auch Kapitel 3). Da kein Substrat mit perfekt angepasster Gitterkonstante zur Verfügung steht, existiert immer eine Gitterfehlanpassung zwischen Schicht und Substrat, die die Schicht beim Wachstum ausgleichen muss. Das Substrat (Dicke ca. $300-500 \mu m$ ) gibt dabei die Gitterkonstante $a$ in der Basalebene vor, während die Schicht in $c$-Richtung frei ist. Die Fehlanpassung kann bei dünnen Schichten durch eine Verformung ausgeglichen werden, wie es in Abbildung 2.2a) für den Fall asubstrat $>$ a schicht gezeigt wird. Diese Art des verspannten Wachstums nennt man pseudomorph. Entsprechend der gezeigten Dehnung in $a$-Richtung ist die Schicht in $c$-Richtung gestaucht.

Die Verformungsenergie, die zur Aufrechterhaltung des pseudomorphen Wachstums notwendig ist, steigt linear mit der Schichtdicke. Ab einer gewissen kritischen Dicke ist 


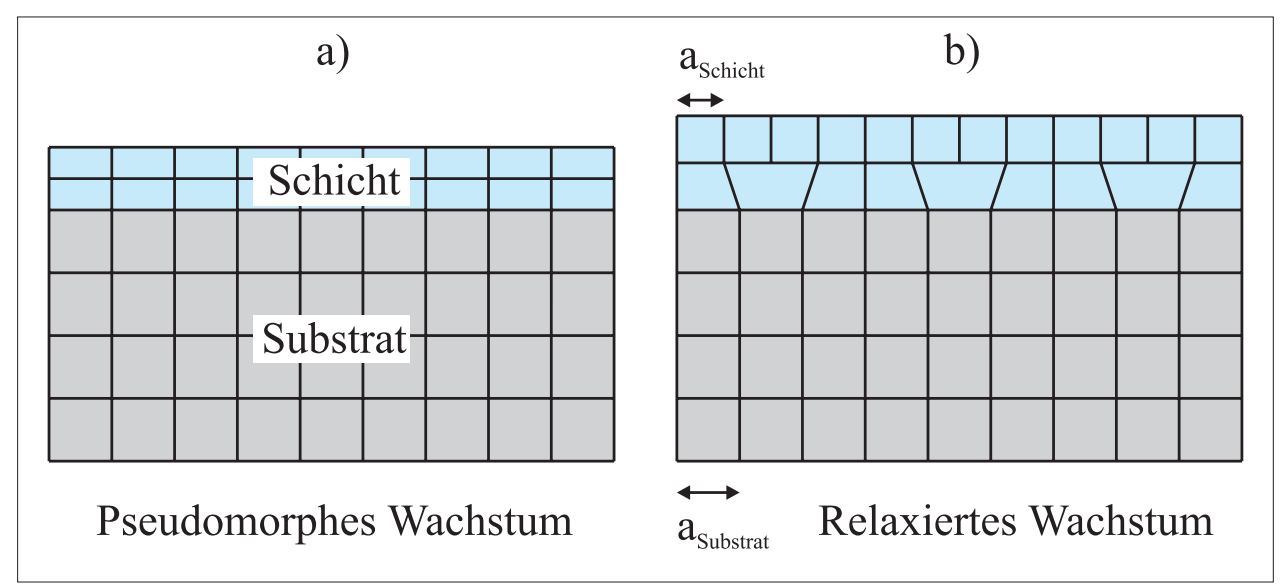

Abbildung 2.2: Ausgleich der Gitterfehlanpassung durch Verformung.

es daher günstiger, die Fehlanpassung durch Versetzungen auszugleichen, deren Bildung eine konstante Energie abhängig von der Gitterfehlanpassung kostet. Dies ist in Abbildung $2.2 \mathrm{~b})$ gezeigt. Ab einer gewissen Entfernung von der Grenzfläche wächst die Schicht dann mit ihrer eigenen Gitterkonstante, man nennt diese Schicht dann relaxiert.

Da das Wachstum grundsätzlich bei hohen Temperaturen stattfindet und die meisten Experimente bei Raum- oder noch niedrigeren Temperaturen durchgeführt werden, spielt auch die thermische Ausdehnung eine wichtige Rolle. Wieder gibt das Substrat durch seine große Dicke hier vor, welche thermische Ausdehnung (bzw. Stauchung) beim Abkühlen der Probe auftreten wird. Die Differenz der thermischen Ausdehnungen von Substrat und Schicht werden also als zusätzliche Verspannungen in der Probe auftreten, die im Fall der typischen Substrate für GaN relativ groß werden können (siehe Kapitel 3). Man kann also davon ausgehen, dass in jeder epitaktisch gewachsenen Schicht eine Restverspannung vorhanden ist, die je nach Substrat kompressiv (gestaucht) oder tensil (gedehnt) in der Basalebene ist.

\subsubsection{Elektronische Eigenschaften}

In Abbildung 2.3 ist die erste Brillouin-Zone von GaN nach [21] abgebildet. $\Gamma$ bezeichnet das Zentrum der Brillouin-Zone, andere wichtige Punkte hoher Symmetrie sind ebenfalls vermerkt.

Abbildung 2.4 zeigt die elektronische Bandstruktur von GaN entlang wichtiger Symmetrierichtungen (siehe Abbildung 2.3). Man erkennt die direkte Bandlücke am $\Gamma$-Punkt, die bei Raumtemperatur etwa $3.44 \mathrm{eV}$ beträgt und nahe dem absoluten Nullpunkt auf etwa $3.50 \mathrm{eV}$ anwächst. Das Leitungsbandminimum wird im Wesentlichen aus den s-Orbitalen des Galliums gebildet, man nennt es daher auch $s$-artig. Im Gegensatz dazu wird das Valenzbandmaximum als $p$-artig beschrieben, da sein Charakter hauptsächlich dem der $p$-Orbitale des Stickstoffs entspricht. In einem vereinfachten Bild sind die drei $p$-Orbitale äquivalent und damit energetisch entartet. Bei genauerer Betrachtung allerdings müssen Effekte berücksichtigt werden, die diese Entartung aufheben. 


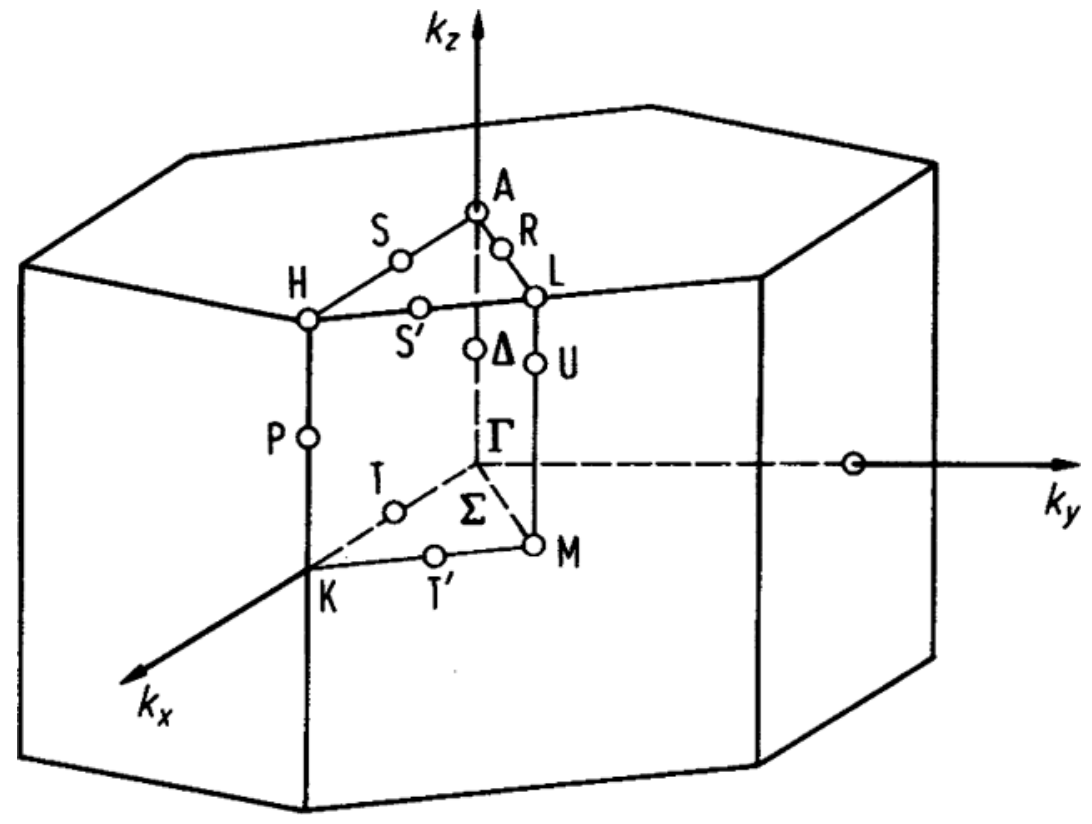

Abbildung 2.3: Erste Brillouin-Zone eines Wurtzit-Kristalls (aus [21])

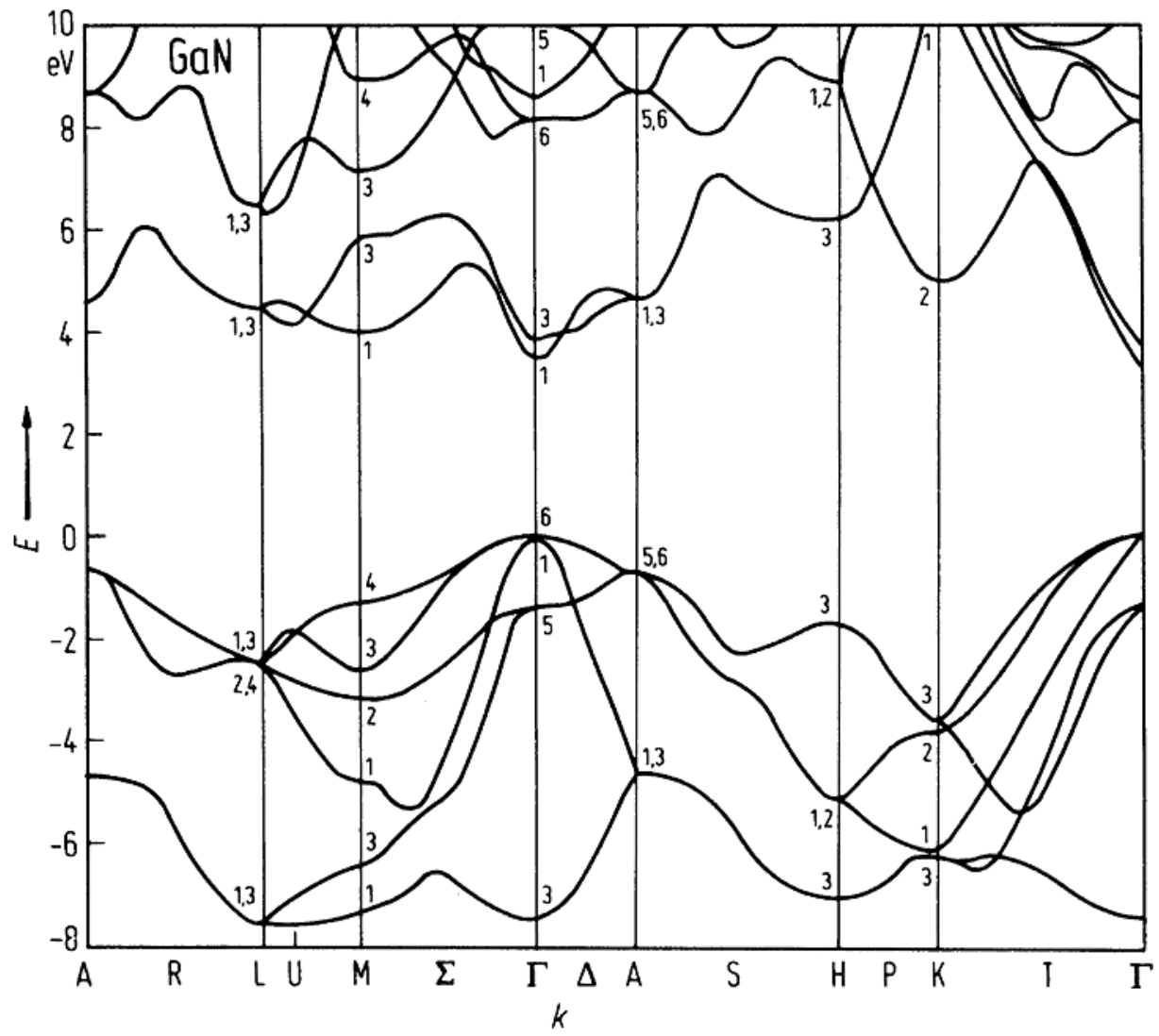

Abbildung 2.4: Elektronische Bandstruktur von GaN ohne Spin-Bahn-Wechselwirkung nach [21] 
In Abbildung 2.5 wird deutlich, welche Effekte für diese Aufspaltung verantwortlich sind. Zum Einen sorgt das Kristallfeld im Wurtzit für die Abspaltung eines Valenzbandes, da in der hexagonalen Symmetrie die c-Achse nicht mehr identisch mit der Basalebene ist. Das nach wie vor zweifach entartete Band mit Symmetrie $\Gamma_{6}$ wird von den $p_{x^{-}}$und $p_{y}$-Orbitalen gebildet, das Band mit Symmetrie $\Gamma_{1}$ von den $p_{z}$-Orbitalen. Des Weiteren führt die Spin-Bahn-Wechselwirkung zu einer weiteren Aufhebung der Entartung. Dieser zweite Effekt ist umso stärker ausgeprägt, je höher die Ordnungszahl eines Atoms ist. Daher ist er bei den Nitriden sehr klein im Vergleich zu anderen III-V-Halbleitern, da die Valenzbandzustände überwiegend am Anion lokalisiert sind.

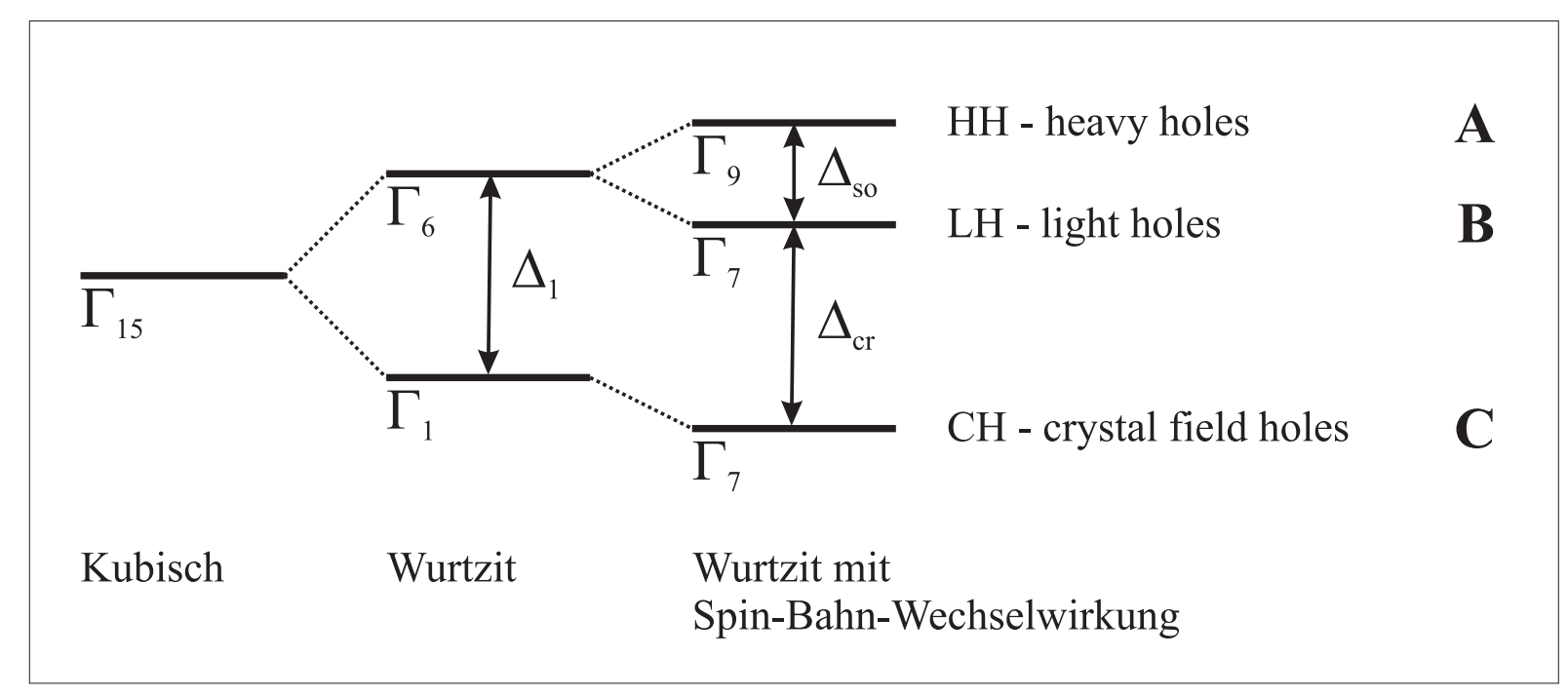

Abbildung 2.5: Aufspaltung der Valenzbänder in GaN durch Spin-Bahn-Wechselwirkung und das Kristallfeld

Es ergeben sich also drei Valenzbänder, die in der Notation der Doppelgruppe (die den Orts- und Spinanteil der Wellenfunktionen berücksichtigt) die Symmetrien $\Gamma_{9}, \Gamma_{7}$ und $\Gamma_{7}$ besitzen, wobei das $\Gamma_{9}$-Band in GaN energetisch am höchsten liegt. Nach ihrer Krümmung im $k$-Raum und der daraus folgenden effektiven Masse werden die Bänder auch als Schwerloch-(HH-), Leichtloch-(LH-) und Kristallfeldloch-(CH-) Bänder bezeichnet. In der Optik werden sie üblicherweise mit den Buchstaben A, B und C abgekürzt, in der Reihenfolge ihrer energetischen Lage. 


\subsection{Kristallfeldtheorie}

Im Rahmen dieser Arbeit wurden die optischen Eigenschaften von Mn in GaN untersucht. Ersetzt ein Mn-Atom ein Ga-Atom, so ist es einem so genannten Kristallfeld der umgebenden Gitteratome ausgesetzt, das im Wesentlichen ein Coulomb-Feld der Valenzelektronen und Kerne ist. Dessen Einfluss auf die elektronischen Eigenschaften von Mn soll in diesem Kapitel genauer geklärt werden.

\subsection{1 Übergangsmetalle in tetraedrischer und oktaedrischer Umgebung}

Das freie Ion Übergangsmetalle besitzen teilweise gefüllte $d$-Orbitale, die innerhalb der jeweiligen Periode aufgefüllt werden. Die $d$-Orbitale sind charakterisiert durch einen Bahndrehimpuls $l=2$ und entsprechender $z$-Komponente $m_{l}=-2 \ldots 2$. Unter Berücksichtigung des Elektronenspins mit $s=1 / 2$ und $m_{s}= \pm 1 / 2$ kann man die neuen Größen Gesamtdrehimpuls $\vec{j}=\vec{l}+\vec{s}$ und seine $z$-Komponente $m_{j}=-j \ldots j$ einführen. Das Auffüllen der $d$-Orbitale mit Elektronen folgt nun dem Pauli-Prinzip und den Hundschen Regeln, die letztendlich Ausdruck der Spin-Bahn- und Coulomb-Wechselwirkung sind. Der Hamilton-Operator solch eines Mehr-Elektronen-Systems lässt sich wie folgt schreiben:

$$
\begin{aligned}
\hat{H}_{F I}=\hat{H}_{0} & +\hat{H}^{\prime}+\hat{H}_{s o} \\
\hat{H}_{0} & =\sum_{i} \frac{\vec{p}_{i}^{2}}{2 m}+V^{\prime}\left(r_{i}\right) \\
\hat{H}^{\prime} & =\sum_{i>j} \frac{e^{2}}{4 \pi \epsilon_{0} r_{i j}} \\
\hat{H}_{s o} & =\sum_{i} \zeta\left(r_{i}\right) \vec{l}_{i} \cdot \vec{s}_{i} .
\end{aligned}
$$

Dabei ist das Potential $V^{\prime}$ in $\hat{H}_{0}$ das Zentralpotential, dass vom Atomkern plus den inneren Elektronen auf alle äußeren Elektronen wirkt, die hier ausschließlich betrachtet werden. Die Summationen werden genau über diese äußeren $(d-)$ Elektronen durchgeführt.

$\hat{H}^{\prime}$ ist der Energiebeitrag der Coulomb-Wechselwirkung zwischen den $d$-Elektronen. Durch diesen Term sind die $l_{i}$ keine guten Quantenzahlen mehr und man muss für eine quantenmechanische Beschreibung zu $\vec{L}=\sum_{i} \vec{l}_{i}$ übergehen. Entsprechend wird $\vec{S}=\sum_{i} \overrightarrow{s_{i}}$ definiert und analog dem Ein-Elektronen-Fall der Gesamtdrehimpuls $\vec{J}$. Auch hier werden wieder die jeweiligen Eigenzustände der $z$-Komponenten mit $M_{L}, M_{S}$ und $M_{J}$ bezeichnet. Die Energie eines Zustandes $\left|L S M_{L} M_{S}\right\rangle$ hängt nur von $L$ und $S$ ab, weswegen auch eine Linearkombination von $M_{L}$ und $M_{S}$ benutzt werden kann, die Eigenzuständen von $\hat{\vec{J}}^{2}$ und $\hat{J}_{z}$ entspricht. Die Koeffizienten dieser Linearkombinationen werden als Clebsch-Gordan Koeffizienten bezeichnet, die sich aus der Projektion $\left\langle L S M_{L} M_{S} \mid L S J M_{J}\right\rangle$ ergeben. Unter Berücksichtigung der Coulomb-Wechselwirkung besitzt also jeder so genannte $L S$-Zustand eine bestimmte Energie, ist aber noch $(2 L+1)(2 S+1)$-fach entartet. 
Der letzte Term in Gleichung 2.2, $\hat{H}_{\text {so }}$, beschreibt die Spin-Orbit-Wechselwirkung mit dem Kopplungsparameter $\zeta$. Er wird um so größer, je höher die Ordnungszahl des betrachteten Atoms ist. Der Fall der so genannten $j$ - $j$-Kopplung wird für sehr schwere Atome verwendet, hierbei koppeln $\overrightarrow{l_{i}}$ und $\overrightarrow{s_{i}}$ zuerst zu den $\overrightarrow{j_{i}}=\overrightarrow{l_{i}}+\overrightarrow{s_{i}}$ und daraus wird dann $\vec{J}=\sum_{i} \overrightarrow{j_{i}}$ gebildet. Dies soll hier nicht weiter betrachtet werden, da $\hat{H}_{s o}$ für die Übergangsmetalle der vierten Periode als klein gegenüber den anderen Termen betrachtet werden kann. In diesem Fall kann das Problem in der so genannten $L S$-Kopplung betrachtet werden, wo $\overrightarrow{l_{i}}$ und $\overrightarrow{s_{i}}$ zuerst zu $\vec{L}$ und $\vec{S}$ koppeln, bevor diese beiden zu $\vec{J}=\vec{L}+\vec{S}$ gekoppelt werden. In der so genannten Russel-Saunders-Näherung wird das Mischen unterschiedlicher $L S$-Terme zu den gleichen $J M_{J}$-Zuständen vernachlässigt, so dass $\left|L S J M_{J}\right\rangle$ als Eigenzustand zu $\hat{H}_{s o}$ und damit zu $\hat{H}_{F I}$ verwendet werden kann. Es ergibt sich damit ein Energiespektrum, das in die unterschiedlichen $L S$-Niveaus aufgespalten ist, die wiederum nach $J$ aufspalten. Die Zustände werden in der Spektroskopie häufig mit der Schreibweise ${ }^{2 S+1} L_{J}$, den Termsymbolen, beschrieben. Für $L=0,1,2, \ldots$ werden dabei die Buchstaben $S, P, D, \ldots$ verwendet.

Kristallfeld Wird ein Atom in einen Festkörper eingebaut, so ist er in einer ganz bestimmten Symmetrie von anderen Atomen umgeben. Das gleiche gilt für chemische Komplexe, in denen ein Zentralkation von mehreren Liganden umgeben ist. Um die Auswirkung dieser Umgebung auf das zu betrachtende Atom zu beschreiben, wurde die Kristallfeldbzw. Ligandenfeld-Theorie entwickelt, die nun kurz vorgestellt werden soll. Der HamiltonOperator des freien Ions (Gleichung 2.2) wird folgendermaßen erweitert:

$$
\begin{aligned}
\hat{H} & =\hat{H}_{F I}+\hat{H}_{c} \\
& =\hat{H}_{0}+\hat{H}^{\prime}+\hat{H}_{s o}+\hat{H}_{c} .
\end{aligned}
$$

Dabei sind $\hat{H}_{0}, \hat{H}^{\prime}$ und $\hat{H}_{s o}$ wie in den Gleichungen $2.3-2.5$ definiert. $\hat{H}_{c}$ beschreibt genau das elektrostatische Kristallfeld, dass auf die äußeren Elektronen wirkt. In der einfachsten Näherung werden die nächsten Nachbarn durch Punktladungen charakterisiert und weiter entfernte Nachbarn vernachlässigt. Dann lässt sich der Hamilton-Operator des Kristallfeldes wie

$$
\hat{H}_{c}=\frac{1}{4 \pi \epsilon_{0}} \sum_{i} \sum_{l} \frac{Z_{l} e^{2}}{\left|\vec{R}_{l}-\vec{r}_{i}\right|}
$$

schreiben. Dabei ist $\vec{R}_{l}$ die Position des $l$-ten Nachbarions mit effektiver Ladung $q_{l}=$ $-Z_{l} e$ und $i$ die Zahl der Elektronen. Es ist nun möglich, den Term $\left|\vec{R}_{l}-\vec{r}_{i}\right|^{-1}$ durch die Kugelfunktionen $Y_{k}^{t}(\theta, \phi)$ auszudrücken, so dass man

$$
\hat{H}_{c}\left(\vec{r}_{i}\right)=\frac{1}{4 \pi \epsilon_{0}} \sum_{l} \sum_{k=0}^{\infty} \sum_{t=-k}^{k}\left(\frac{4 \pi}{2 k+1}\right)^{\frac{1}{2}} \frac{Z_{l} e^{2}}{a_{l}^{k+1}} r_{i}^{k} C_{t}^{(k)}\left(\theta_{i}, \phi_{i}\right) Y_{k}^{t}\left(\theta_{l}, \phi_{l}\right)
$$

für das $i$-te Elektron erhält [25]. Dabei gilt

$$
C_{t}^{(k)}(\theta, \phi)=\left(\frac{4 \pi}{2 k+1}\right)^{\frac{1}{2}} Y_{k}^{t}(\theta, \phi)
$$


Bei der Berechnung der Effekte des Kristallfeldes werden also Matrixelemente der Form

$$
\left\langle n^{\prime} l^{\prime} m_{l}^{\prime}\left|r^{k} C_{t}^{(k)}\right| n l m_{l}\right\rangle=\left\langle R_{n^{\prime} l^{\prime}}^{\prime}\left|r^{k}\right| R_{n l}^{\prime}\right\rangle\left\langle Y_{l^{\prime}}^{m_{l}^{\prime}}\left|C_{t}^{(k)}\right| Y_{l}^{m_{l}}\right\rangle
$$

auftreten, wobei $R_{n l}^{\prime}$ die Radialfunktionen des modifizierten Wasserstoff-Problems sind. Die Werte dieser Integrale sind bekannt und können beispielsweise bei [26] (und dortige Referenzen) nachgeschlagen werden. Wie dort dargelegt, ist der Winkelteil dabei nur unter bestimmten Bedingungen ungleich Null, so dass sich deutliche Vereinfachungen ergeben können.

a)

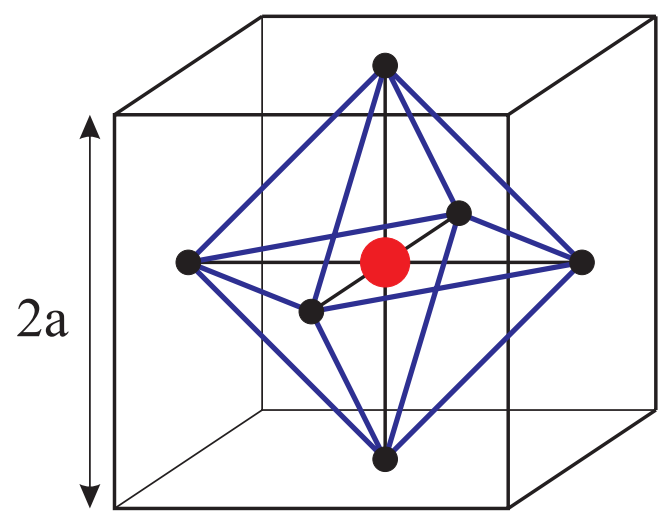

b)

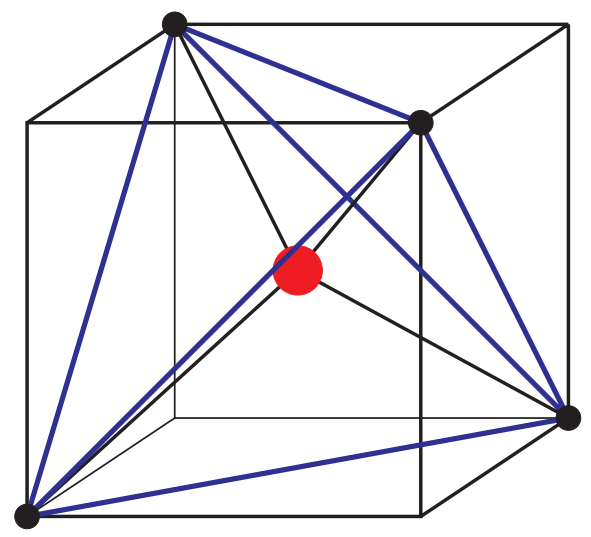

Abbildung 2.6: Anordnung der Ionen bei oktaedrischer (links) und tetraedrischer (rechts) Koordination relativ zum kartesischen Koordinatensystem. In Rot ist das zentrale Kation gezeichnet, die umgebenden Anionen in Schwarz. Die Bindungslänge beträgt $a$ im Oktaeder- und $\sqrt{3} a$ im Tetraeder.

Diese Überlegungen können nun auf ein einfaches Problem, ein einzelnes $d$-Elektron in einem oktaedrischen Feld, angewandt werden. Eine entsprechende Kristallstruktur ist schematisch in Abbildung 2.6a) zu sehen. Das Ion im Mittelpunkt wird dabei von sechs anderen Ionen im gleichen Abstand $a$ jeweils entlang der Koordinatenachsen umgeben. Da das Zentrum in der Form eines regelmäßigen Oktaeders umgeben ist, spricht man von oktaedrischer Symmetrie, kurz $O_{h}$. Das Kristallfeld lässt sich in diesem Fall wie

$$
V(x, y, z)=V_{x}+V_{y}+V_{z}
$$

ausdrücken, wobei

$$
V_{x}=-\frac{Z e}{4 \pi \epsilon_{0}}\left[\frac{1}{\left(r^{2}+a^{2}-2 a x\right)^{\frac{1}{2}}}+\frac{1}{\left(r^{2}+a^{2}+2 a x\right)^{\frac{1}{2}}}\right]
$$

ist und $V_{y}$ und $V_{z}$ analog dargestellt werden können. Damit erhält man den HamiltonOperator $\hat{H}_{c}^{O_{h}}=-e V$ des oktaedrischen Kristallfeldes. Er kann nun durch eine TaylorEntwicklung und das Benutzen von Kugelfunktionen in die entsprechende Form von Gleichung 2.8 gebracht werden, so dass sich

$$
\hat{H}_{c}^{O_{h}}=\frac{Z e^{2}}{4 \pi \epsilon_{0}}\left[\frac{6}{a}+\frac{7 r^{4}}{2 a^{5}}\left\{C_{0}^{(4)}(\theta, \phi)+\left(\frac{5}{14}\right)^{\frac{1}{2}}\left(C_{4}^{(4)}(\theta, \phi)+C_{-4}^{(4)}(\theta, \phi)\right)\right\}\right]+O\left(r^{6}\right)
$$


ergibt. Der erste Term ergibt nur eine Verschiebung der Energieachse und wird im Weiteren vernachlässigt. Die höheren Ordnungen in $r$ tragen auf Grund der bei Gleichung 2.10 angesprochenen Bedingungen (siehe [26]) im Falle von $d$-Elektronen nicht bei, so dass nun die Matrixelemente der Zustände $\left|3 \mathrm{~d} m_{l}\right\rangle$ mit dem relevanten Teil von $\hat{H}_{c}^{O_{h}}$ berechnet werden kann. So ergibt sich beispielsweise für den Zustand $|3 \mathrm{~d} 0\rangle$ :

$$
\left\langle 3 \mathrm{~d} 0\left|\hat{H}_{c}^{O_{h}}\right| 3 \mathrm{~d} 0\right\rangle=6 D q
$$

mit

$$
D=\frac{1}{4 \pi \epsilon_{0}} \frac{35 Z e^{2}}{4 a^{5}} \quad, \quad q=\frac{2}{105}\left\langle R_{3 d}^{\prime}\left|r^{4}\right| R_{3 d}^{\prime}\right\rangle .
$$

$D$ und $q$ treten grundsätzlich als Produkt auf, welches gleichzeitig als Maß für die Stärke des Kristallfeldes benutzt werden kann. Man kann nun alle Matrixelemente der Zustände $\left|3 \mathrm{~d} m_{l}\right\rangle$ berechnen und damit den Hamilton-Operator $\hat{H}_{c}^{O_{h}}$ als Matrix schreiben:

$$
\begin{aligned}
& 3 \mathrm{~d} 2 \\
& 3 \mathrm{~d} 2 \\
& 3 \mathrm{~d} 1 \\
& 3 \mathrm{~d} 0 \\
& 3 \mathrm{~d}-1 \\
& 3 \mathrm{~d}-2
\end{aligned}\left(\begin{array}{ccccc}
D q & 0 & 0 & 0 & 5 D q \\
0 & -4 D q & 0 & 0 & 0 \\
0 & 0 & 6 D q & 0 & 0 \\
0 & 0 & 0 & -4 D q & 0 \\
5 D q & 0 & 0 & 0 & D q
\end{array}\right) .
$$

Man sieht sofort, dass $|3 \mathrm{~d} 0\rangle$ und $|3 \mathrm{~d} \pm 1\rangle$ bereits Eigenzustände des Operators $\hat{H}_{c}^{O_{h}}$ sind, während $|3 \mathrm{~d} \pm 2\rangle$ gemischt werden. Nach der Diagonalisierung ergeben sich genau zwei Eigenzustände zum Eigenwert $6 D q$ und drei Eigenzustände zum Eigenwert $-4 D q$. Üblicherweise wählt man folgende Darstellung:

$$
\begin{aligned}
\phi_{e u} & =|3 \mathrm{~d} 0\rangle=R_{3 d}^{\prime}(r)\left(\frac{5}{4 \pi}\right)^{\frac{1}{2}}\left(\frac{3 z^{2}-r^{2}}{2 r^{2}}\right) \\
\phi_{e v} & =\frac{1}{\sqrt{2}}(|3 \mathrm{~d} 2\rangle+|3 \mathrm{~d}-2\rangle)=R_{3 d}^{\prime}(r)\left(\frac{15}{4 \pi}\right)^{\frac{1}{2}}\left(\frac{x^{2}-y^{2}}{2 r^{2}}\right) \\
\phi_{t_{2} \xi} & =\frac{i}{\sqrt{2}}(|3 \mathrm{~d} 1\rangle+|3 \mathrm{~d}-1\rangle)=R_{3 d}^{\prime}(r)\left(\frac{15}{4 \pi}\right)^{\frac{1}{2}}\left(\frac{y z}{r^{2}}\right) \\
\phi_{t_{2} \eta} & =-\frac{1}{\sqrt{2}}(|3 \mathrm{~d} 1\rangle-|3 \mathrm{~d}-1\rangle)=R_{3 d}^{\prime}(r)\left(\frac{15}{4 \pi}\right)^{\frac{1}{2}}\left(\frac{x z}{r^{2}}\right) \\
\phi_{t_{2} \zeta} & =-\frac{i}{\sqrt{2}}(|3 \mathrm{~d} 2\rangle-|3 \mathrm{~d}-2\rangle)=R_{3 d}^{\prime}(r)\left(\frac{15}{4 \pi}\right)^{\frac{1}{2}}\left(\frac{x y}{r^{2}}\right)
\end{aligned}
$$

Die beiden Wellenfunktionen in 2.17 werden als die $e_{g}$-Orbitale des Kristallfeldes bezeichnet. Sie gehören zum Eigenwert $6 D q$. Die drei Wellenfunktionen in 2.18 dagegen werden als $t_{2 g}$-Orbitale bezeichnet, die den Eigenwert $-4 D q$ besitzen. Damit werden die fünffach entarteten $d$-Niveaus durch das oktaedrische Kristallfeld in ein dreifach entartetes 


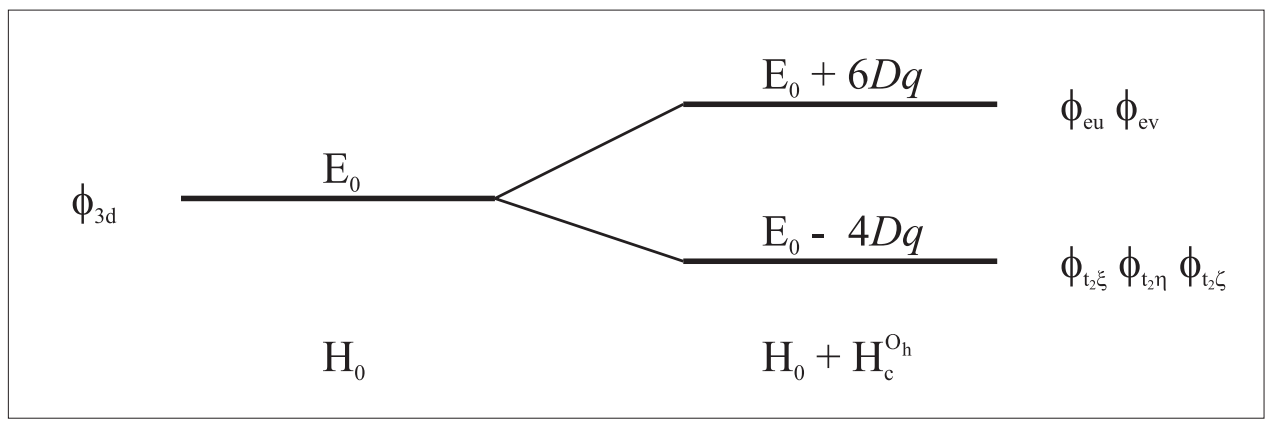

Abbildung 2.7: Aufspaltung der $d$-Orbitale im oktaedrischen Kristallfeld. Ein $d$-Elektron hat die Energie $E_{0}$ im Zentralfeld von Kern und inneren Elektronen, bei Anwesenheit des Kristallfeldes gibt es zwei Möglichkeiten, die sich um $10 D q$ unterscheiden.

$t_{2 g}$-Niveau und ein zweifach entartetes $e_{g}$-Niveau aufgespalten, wie in Abbildung $2.7 \mathrm{zu}$ sehen.

Dieses einfache Modell beschreibt die experimentellen Befunde qualitativ sehr gut, da es die Symmetrie korrekt wiedergibt. So sind die Winkelabhängigkeiten in Gleichung 2.13 auch bei einer exakten Beschreibung identisch. Quantitativ ist die Näherung der Nachbarionen als Punktladungen allerdings unhaltbar, so dass die theoretischen Werte für $D$ und $q$ in den Gleichungen 2.15 nicht mit der Realität übereinstimmen. Eine etwas bessere Näherung ergibt sich durch Berücksichtigung des kovalenten Charakters der Bindungen. $10 D q$ kann aber auch einfach durch spektroskopische Messungen ermittelt werden und damit das Modell in sehr gute Übereinstimmung mit den Experimenten gebracht werden.

Es soll an dieser Stelle angemerkt werden, dass die Eigenzustände im oktaedrischen Kristallfeld wie sie in den Gleichungen 2.17 und 2.18 definiert wurden, genau den üblichen Bildern der $d$-Orbitale entsprechen, die man in den meisten Physik- und ChemieLehrbüchern findet und wie sie in Abbildung 2.8 gezeigt werden. Die beiden $e_{g}$-Orbitale $\phi_{e u}$ und $\phi_{e v}$ sind dabei genau in Richtung der Koordinatenachsen ausgedehnt und erfahren daher eine Energieerhöhung, da genau dort die elektronenreichen Anionen liegen. Die $t_{2 g^{-}}$ Orbitale $\phi_{t_{2} \xi}, \phi_{t_{2} \eta}$ und $\phi_{t_{2} \zeta}$ liegen in den Flächendiagonalen, wo keine direkten Nachbarn sind, daher ist ihre Energie im Oktaederfeld erniedrigt.

Betrachtet man nun ein Kristallfeld mit Tetraedersymmetrie $T_{d}$, wie es in Abbildung 2.6b) gezeigt wird und wie es für einen substitutionellen Gitterplatz im Wurtzit typisch ist, so kann man es wieder in den kartesischen Koordinaten schreiben. Der Abstand der Nachbarn vom Zentrum ist dabei $\sqrt{3} a$. Nach entsprechender Rechnung erhält man analog Gleichung 2.13 folgenden Ausdruck:

$$
\begin{aligned}
\hat{H}_{c}^{T_{d}}= & \frac{Z e^{2}}{4 \pi \epsilon_{0}}\left[\frac{4}{\sqrt{3} a}-\frac{i \sqrt{40} r^{3}}{3(\sqrt{3} a)^{4}}\left(C_{2}^{(3)}(\theta, \phi)-C_{-2}^{(3)}(\theta, \phi)\right)\right] \\
& -\frac{Z e^{2}}{4 \pi \epsilon_{0}}\left[\frac{14 r^{4}}{9(\sqrt{3} a)^{5}}\left\{C_{0}^{(4)}(\theta, \phi)+\left(\frac{5}{14}\right)^{\frac{1}{2}}\left(C_{4}^{(4)}(\theta, \phi)+C_{-4}^{(4)}(\theta, \phi)\right)\right\}\right] \\
& + \text { Terme höherer Ordnung ... }
\end{aligned}
$$

Der konstante Term kann wie im oktaedrischen Fall vernachlässigt werden. Der Term in 


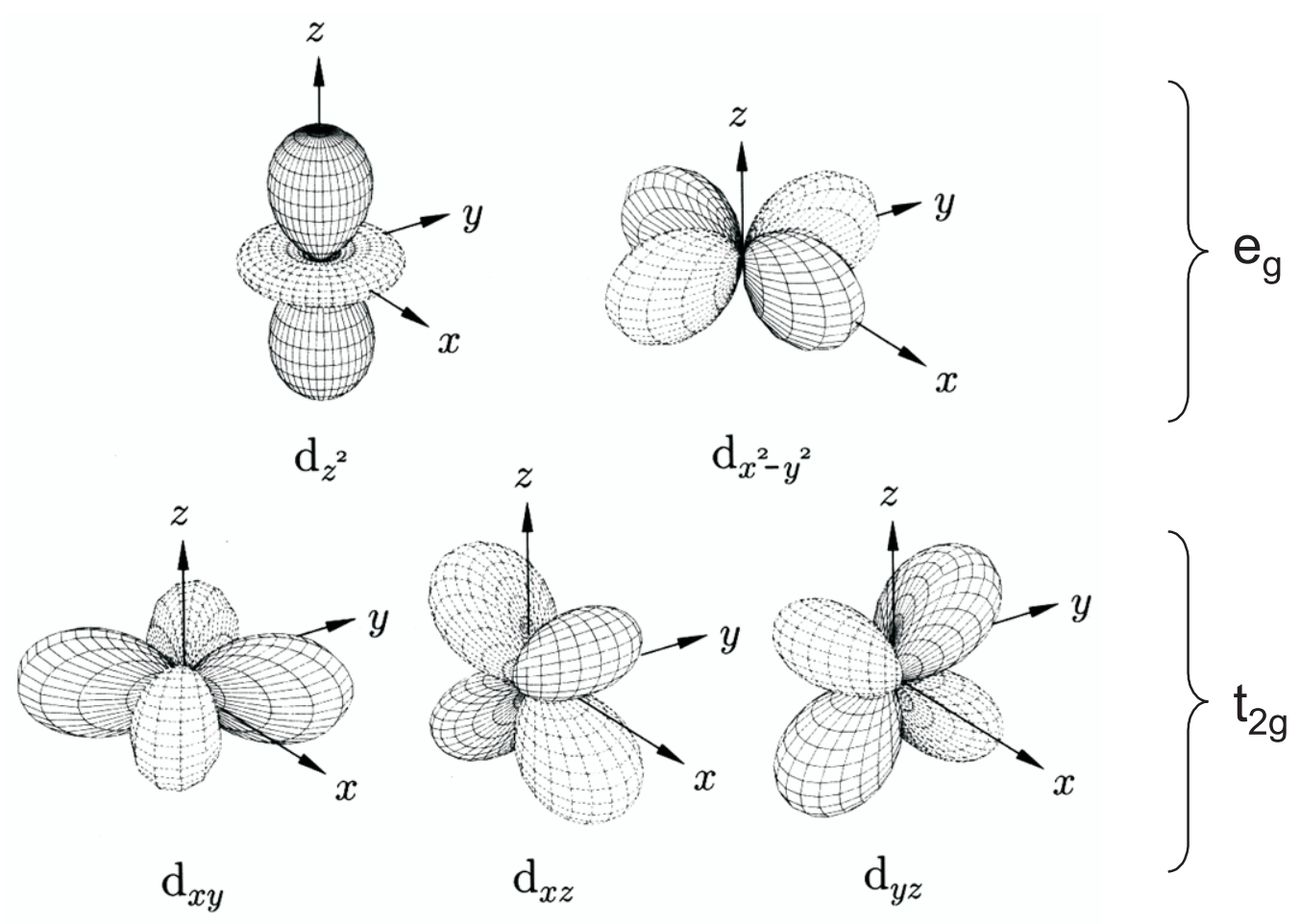

Abbildung 2.8: d-Orbitale im Kristallfeld.

$r^{3}$ tritt auf Grund der fehlenden Inversionssymmetrie im Tetraederfeld auf. Allerdings liefert er keine Beiträge für Matrixelemente zwischen Zuständen mit gleichen Haupt- und Nebenquantenzahlen, so dass er nicht zur Kristallfeldaufspaltung der $d$-Elektronen beiträgt. Durch sie Symmetrieerniedrigung fällt der Index $g$ (für „gerade“, es existiert auch $u$ für ungerade Funktionen) weg und man spricht nur noch von $e$ - und $t_{2}$-Orbitalen. Wie im Oktaederfeld ist also auch im Tetraederfeld nur der $r^{4}$-Term relevant, der noch dazu exakt die gleichen Abhängigkeiten zeigt. Damit sind alle Aussagen bezüglich Symmetrie und Wellenfunktionen bis auf einen konstanten Faktor vom oktaedrischen auf den tetraedrischen Fall übertragbar. Durch das unterschiedliche Vorzeichen sind allerdings die $e$-Orbitale die energetisch niedrigst-liegenden, da sie entlang der Koordinatenachsen liegen und damit den größten Abstand zu den Nachbarionen auf den Raumdiagonalen haben. Die $t_{2}$-Orbitale zeigen entlang der Flächendiagonalen und kommen den Nachbarn daher erheblich näher, so dass sie energetisch höher liegen. Bei gleichen Abständen ist die Kristallfeldaufspaltung im Tetraeder allerdings um den Faktor $\frac{4}{9}$ schwächer als das Oktaederfeld. Es gibt keine Eigenzustände, die genau in Richtung der nächsten Nachbarn oder der größten Lücken des Gitters zeigen.

\subsection{2 $\mathrm{Mn}$ in $\mathrm{GaN}$}

Mangan kann in GaN verschiedene Ladungszustände annehmen, je nach Lage des FermiNiveaus. So wurden von $\mathrm{Mn}^{2+}[27,16]$ bis $\mathrm{Mn}^{4+}[28]$ alle Zustände gefunden, meist aber 
$\mathrm{Mn}^{3+}[16] . \mathrm{Mn}^{4+}$ konnte ausschließlich durch Kodotierung mit $\mathrm{Mg}$ erreicht werden, während $\mathrm{Mn}^{2+}$ zumindest anteilig auch in reinem Material gefunden wurde, das Verhältnis zu $\mathrm{Mn}^{3+}$ durch Si-Kodotierung allerdings erheblich erhöht werden konnte [29].

Der neutrale Ladungszustand für $\mathrm{Mn}_{\text {Ga }}$ ist $\mathrm{Mn}^{3+}$, weshalb er auch für intrinsisches GaMnN erwartet wird. Da allerdings GaN ohne Dotierung durch Rest-Verunreinigungen und elektrisch aktive strukturelle Defekte immer n-leitend ist, erklärt sich der teilweise vorhandene $\mathrm{Mn}^{2+}$-Zustand. Schematisch sind die Umladungsniveaus für $\mathrm{Mn}$ in $\mathrm{GaN}$ in Abbildung 2.9 gezeigt.
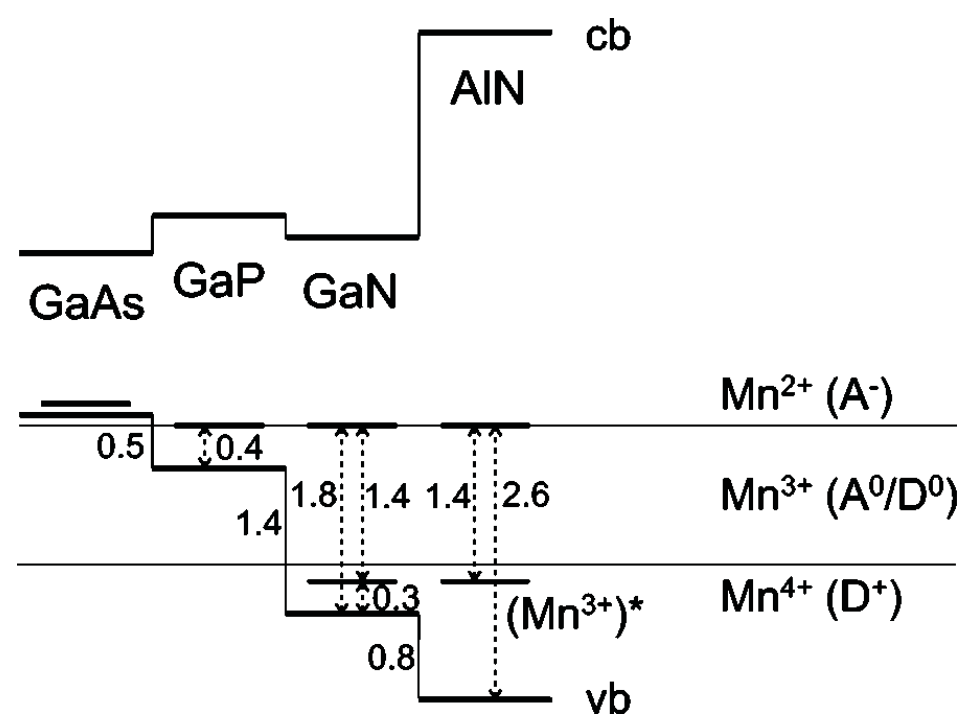

Abbildung 2.9: Umladungsniveaus von $\mathrm{Mn}$ in verschiedenen III-V-Halbleitern nach [16]. Das $\mathrm{Mn}^{4+} / \mathrm{Mn}^{3+}$-Niveau befindet sich $0.3 \mathrm{eV}$, das $\mathrm{Mn}^{3+} / \mathrm{Mn}^{2+}$-Niveau $1.8 \mathrm{eV}$ über dem Valenzbandmaximum. Die durchgezogenen Linien zeigen den universellen Trend der so genannten LangerHeinrich-Regel, nach der es einen festen Zusammenhang zwischen Umladungsniveaus und BandDiskontinuitäten gibt.

Im Folgenden soll der Ladungszustand $\mathrm{Mn}^{3+}$ und die Auswirkungen des Kristallfeldes darauf genauer studiert werden. In diesem Zustand besitzt Mn vier $d$-Elektronen, kurz $d^{4}$. Der Hamilton-Operator wie er in Gleichung 2.6 definiert wurde, enthält jetzt also sowohl einen Coulomb-Term für die Wechselwirkung zwischen den $d$-Elektronen als auch das Kristallfeld mit jeweils vergleichbarer Stärke. Die Spin-Bahn-Wechselwirkung ist deutlich schwächer und kann im ersten Schritt vernachlässigt werden. Zur Lösung dieses Problems ist eine vollständige gruppentheoretische Behandlung sinnvoll, wie sie von Tanabe und Sugano $[30,31,32]$ durchgeführt wurde. Dabei werden die Ein-Elektronen-Wellenfunktionen benutzt (siehe Gleichungen 2.17 und 2.18), die man aus der Diagonalisierung von $\hat{H}_{0}+\hat{H}_{c}$ erhalten hat. Aus diesen werden Kombinationen berechnet und die Matrixelemente mit $\hat{H}^{\prime}$ bestimmt, aus denen sich schließlich die Mehr-Elektronen-Zustände ergeben. Deren Symmetrie entspricht nach wie vor grundsätzlich der des Kristallfeldes, sie werden in der Schreibweise ${ }^{2 S+1} \Gamma$ dargestellt, wobei $\Gamma$ der entsprechenden irreduziblen Repräsentation der Symmetriegruppe des Mehr-Elektronen-Zustands im Kristallfeld entspricht. Der Grundzustand eines $d^{4}$-Systems ohne Kristallfeld wird durch das Termsymbol ${ }^{5} D$ beschrieben. Durch ein tetraedrisches Kristallfeld spaltet er nun in den dreifach entarteten ${ }^{5} T_{2^{-}}$ 
und den doppelt entarteten ${ }^{5} E$-Zustand auf, wobei ${ }^{5} T_{2}$ der neue Grundzustand ist. Man kann sich diese Zustände in diesem Fall mit einem Ein-Elektronen-Bild wie in Abbildung 2.10 gezeigt veranschaulichen.

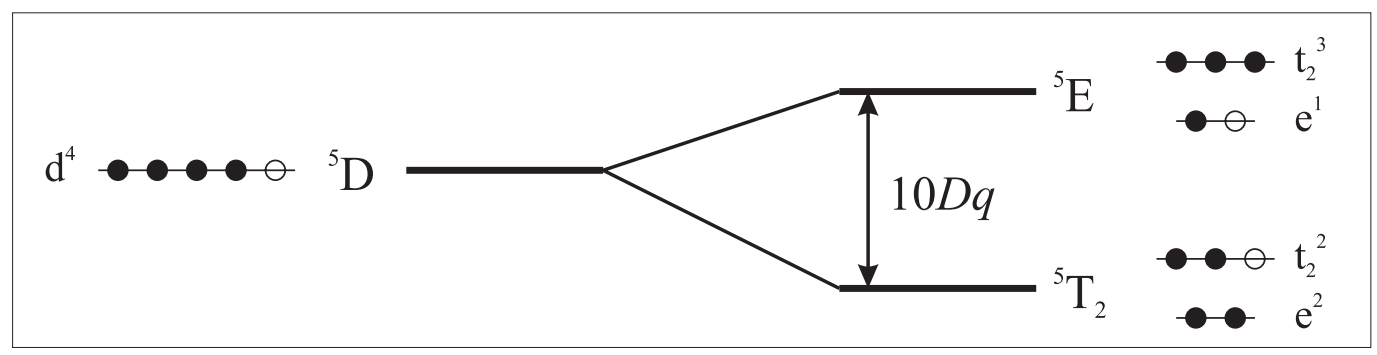

Abbildung 2.10: Aufspaltung des Grundzustandes eines $d^{4}$-Elektronensystems im Tetraederfeld. Rechts die Anordnung der einzelnen Elektronen in den $e$ - und $t_{2}$-Orbitalen.

Der energetische Abstand zwischen ${ }^{5} T_{2}$ und ${ }^{5} E$ entspricht dabei genau $10 D q$, also dem Wert, den man auch im Ein-Elektronen-Bild erwartet hätte. Höhere elektronische Zustände wie ${ }^{1} I$ spalten ebenfalls im Kristallfeld auf, in diesem Fall in die fünf Zustände ${ }^{1} A_{1},{ }^{1} T_{1}$, ${ }^{1} T_{2},{ }^{1} E$ und ${ }^{1} A_{2}$. Deren Energieniveaus zeigen verschiedene Abhängigkeiten von der Kristallfeldstärke $10 D q$ und bei starken Kristallfeldern wird ${ }^{1} A_{1}$ sogar energetisch günstiger als ${ }^{5} T_{2}$ und somit der neue Grundzustand. Dies entspricht dem aus der Chemie bekannten Wechsel von high-spin- zu low-spin-Systemen. Die Aufspaltungen und deren Abhängigkeiten von der Kristallfeldstärke werden in den so genannten Tanabe-Sugano-Diagrammen zusammengefasst, wie es in Abbildung $2.11 \mathrm{zu}$ sehen ist. Die Energie des Grundzustandes wird dabei auf Null gesetzt und alle angeregten Zustände relativ dazu betrachtet. Die senkrechte gestrichelte Linie markiert den Übergang zum neuen Grundzustand ${ }^{1} A_{1}$.

Man sieht außerdem in diesen Diagrammen, dass bei hohen Kristallfeldstärken die Zustände mit der gleichen Verteilung der Elektronen auf $e$ - und $t_{2}$-Orbitale parallel laufen, während bei schwachen Feldern die Coulomb-Wechselwirkung dominiert. Im Grenzfall $D q=0$ erhält man die Zustände des freien Ions.

Gitterverzerrungen und Spin-Bahn-Kopplung GaN in der Wurtzit-Struktur weist auf den Ga-Gitterplätzen keine ideale tetraedrische Umgebung auf; es ist eine uniaxiale Verzerrung vorhanden. Durch diese Verzerrung des Tetraeders in [0001]-Richtung ergibt sich eine kleine trigonale Feldkomponente, die die Symmetrie des Kristallfeldes von $T_{d}$ auf $C_{3 v}$ erniedrigt. Zusätzliche Verspannungen durch das epitaktische Wachstum in [0001]Richtung haben ebenfalls diese Symmetrie und werden damit durch den gleichen Term beschrieben.

Eine weitere Symmetrieerniedrigung ergibt sich aus der Möglichkeit eines so genannten Jahn-Teller-Effektes. Das Jahn-Teller-Theorem [33, 34] sagt aus, dass (außer in linearen Molekülen) durch eine Verzerrung jeder entartete Mehr-Elektronen-Zustand aufgespaltet werden kann. Dies bedeutet gleichzeitig, dass durch diese Verzerrung die Energie minimiert werden kann, da mindestens einer der neuen Zustände energetisch unter dem Ausgangszustand liegen muss. Entartete Zustände sind also in allen nicht-linearen Molekülen und 


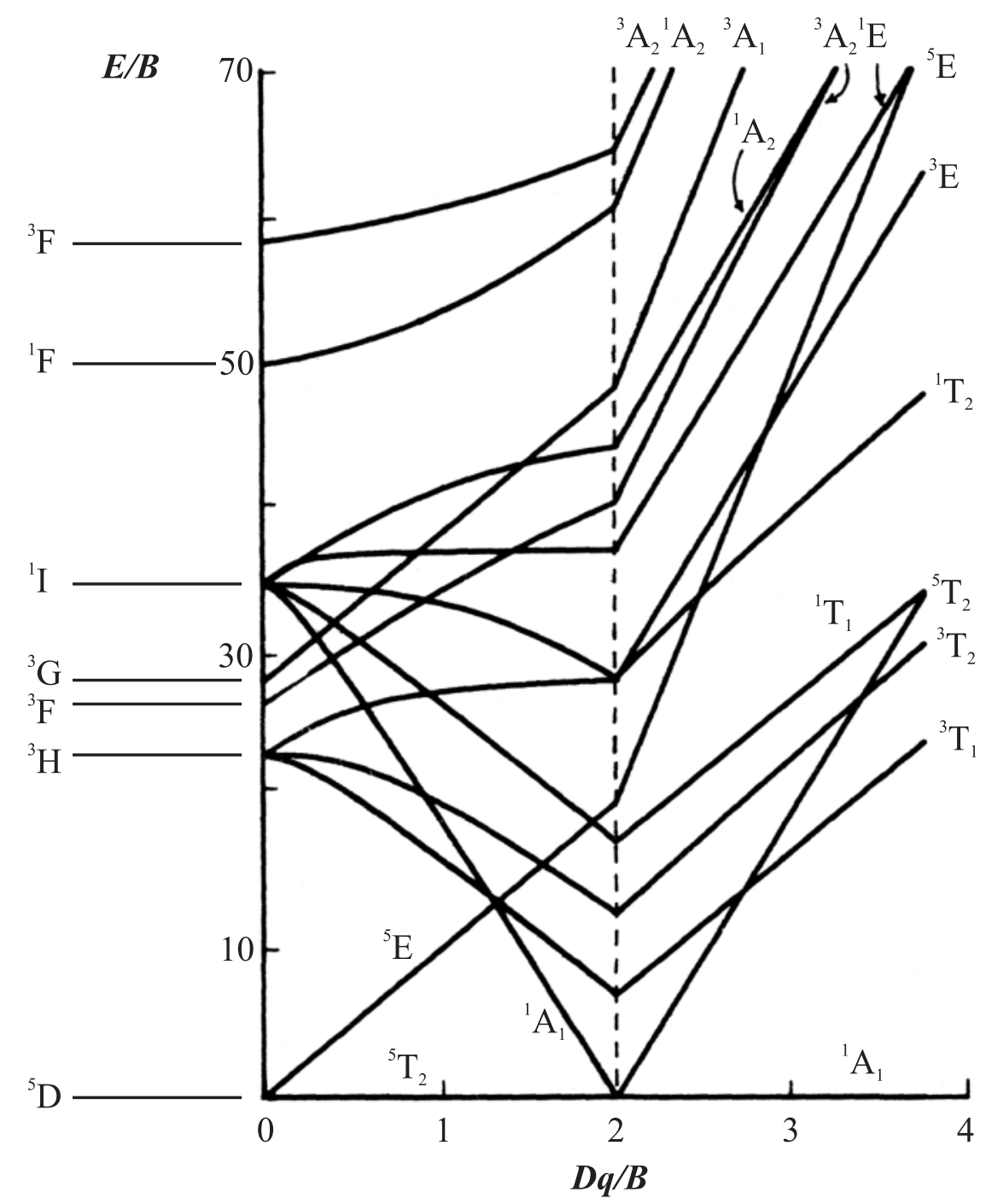

Abbildung 2.11: Tanabe-Sugano Diagramm eines $d^{4}$-Systems im tetraedrischen Kristallfeld (nach [26]). Es werden die Energieniveaus in Abhängigkeit von der Kristallfeldstärke aufgetragen, jeweils normiert auf den Racah-Parameter $B$. Links sind die Niveaus des freien Ions angegeben.

insbesondere Festkörpern instabil. Die Verzerrungen hängen direkt mit den Phononenmoden zusammen, die aus Symmetriegründen die gleichen Normalkoordinaten besitzen. Wolos et al. [35] haben die Absorptionsspektren von $\mathrm{Mn}^{3+}$ in GaN durch Berücksichtigung eines statischen Jahn-Teller-Effektes erklärt, der die Symmetrie von $T_{d}$ auf $D_{2 d}$ erniedrigt (siehe Abbildung 2.12). In dieser Beschreibung koppelt der Grundzustand ${ }^{5} T_{2}$ mit einer Phononenmode in $E$-Symmetrie, was zu Verzerrungen führt, die in den Normalkoordinaten $Q_{\epsilon}$ und $Q_{\theta}$ beschrieben werden. Wie in $D_{2 d}$-Symmetrie erwartet, ergeben sich drei Potentialminima, in denen der Kristall „einfrieren“ kann (siehe Abbildung 2.13).

Das Potential verläuft in der Nähe der Minima parabelförmig, wie es einem harmonischen Oszillator entspricht. Die Potentialdifferenz zwischen den Minima und dem Schnittpunkt bei $Q_{\epsilon}=Q_{\theta}=0$ entspricht der Stabilisierungsenergie dieses Effektes, der so genannten Jahn-Teller-Energie $E_{J T}$. Diese Energie hängt über den Huang-Rhys-Faktor $S$ 

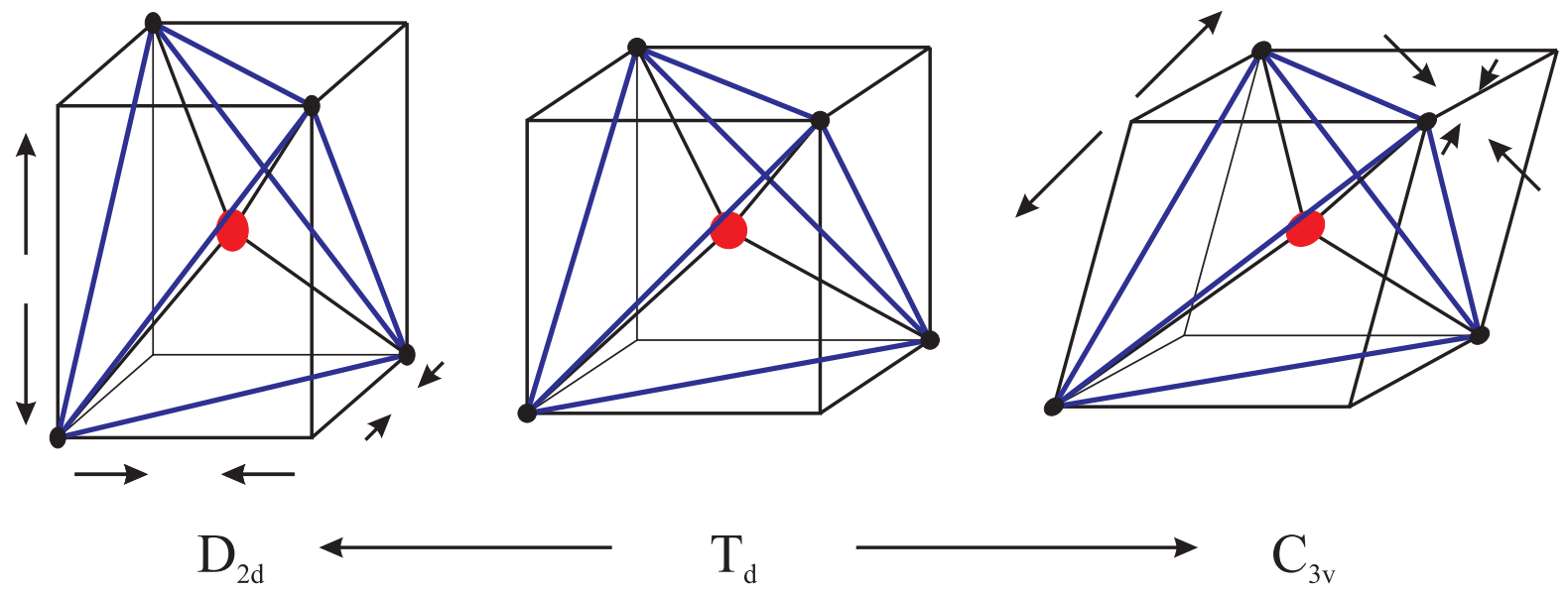

Abbildung 2.12: Abweichungen der Kristallstruktur vom idealen Tetraeder. Links die tetragonale Verzerrung durch einen Jahn-Teller-Effekt nach [35], es gibt dabei drei äquivalente Verzerrungen entlang der kubischen Achsen [001], [010] oder [100]. Rechts die trigonale Verzerrung entlang der kubischen [111]-Achse.

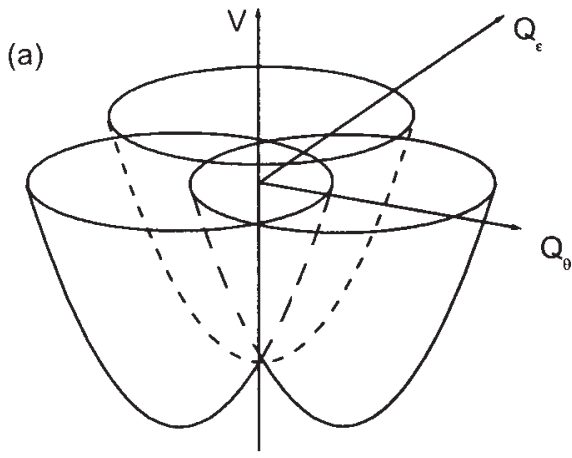

(b)

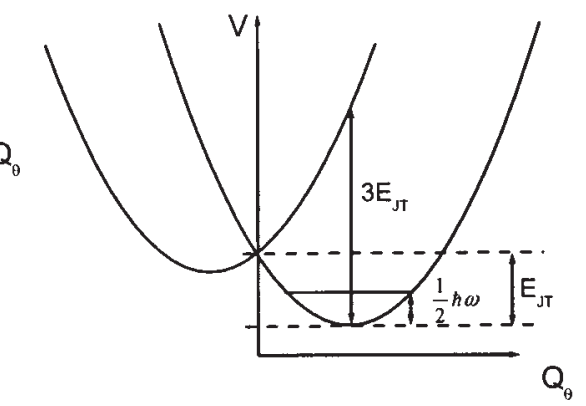

Abbildung 2.13: Potentielle Energie eines $T$-Zustandes gekoppelt mit einer E-Mode aus [35]. $Q_{\epsilon}$ und $Q_{\theta}$ sind die Normalkoordinaten der $E$-Mode mit Energie $\hbar \omega, E_{J T}$ ist die Jahn-Teller-Energie.

mit der Phononenenergie $\hbar \omega$ der gekoppelten Mode zusammen:

$$
E_{J T}=S \hbar \omega
$$

Der Huang-Rhys-Faktor $S$ ist anschaulich betrachtet damit ein Maß für die Verzerrung des Kristalls. Man sieht auch in Abbildung 2.13, dass das orbitale Triplett durch ein vibronisches Triplett ersetzt wurde. Ham [36] hat gezeigt, dass die Nicht-Diagonalelemente des Hamilton-Operators, die nun auf dieses vibronische Triplett wirken, in erster Ordnung abgeschwächt werden. Dies wird durch den Ham-Faktor $\kappa=e^{-3 S / 2}$ beschrieben. Effektiv wird damit der Bahndrehimpuls für diese Elemente abgeschwächt: $L_{e f f}=\kappa L$.

Sowohl tetragonale als auch trigonale Verzerrungen sind in Abbildung 2.12 bildlich dargestellt. Solange diese Verzerrungen klein gegenüber der tetraedrischen Komponente des Kristallfeldes ist, können sie störungstheoretisch berücksichtigt werden. Alleine aus der Symmetrie und der entsprechenden gruppentheoretischen Behandlung folgt aber bereits, welche Entartungen aufgehoben werden. So wird bei tetragonaler Verzerrung der Grundzustand ${ }^{5} T_{2}$ in zwei Niveaus der Symmetrien ${ }^{5} B_{2}$ (der neue Grundzustand) und ${ }^{5} E$ 
aufspalten. Der angeregte Zustand ${ }^{5} E$ des unverzerrten Falles wird $\mathrm{zu}^{5} A_{1}$ und ${ }^{5} B_{1}$. Im trigonalen Feld bleibt die Entartung des ${ }^{5} E$-Zustandes erhalten, nur der Grundzustand spaltet $\mathrm{zu}{ }^{5} E$ und ${ }^{5} A_{1}$ auf.

Ein Effekt, der bisher unberücksichtigt blieb, ist die Spin-Bahn-Wechselwirkung. Sie ist typischerweise wesentlich schwächer als das Kristallfeld und wird deshalb zunächst vernachlässigt. Hier spielt nun zum ersten Mal der Spin der Elektronen eine Rolle. Im freien Ion ergibt sich die bekannte Aufspaltung nach $J$, wie bereits beschrieben. In Verbindung mit dem Kristallfeld wird auch hier wieder eine gruppentheoretische Beschreibung benutzt und aus den einfachen Symmetriegruppen der Bahndrehimpuls-Zustände werden die so genannten Doppelgruppen. Letztendlich werden damit weitere Entartungen aufgehoben und die Feinstruktur der experimentell beobachteten Linien wird erklärbar.

dynamischer Jahn-Teller-Effekt Die obige Beschreibung des Jahn-Teller-Effektes beruht auf dem statischen Fall, was bedeutet, dass die lokale Umgebung des $\mathrm{Mn}^{3+}$ statisch verzerrt wird und dann in einem der Potentialminima aus Abbildung 2.13 als neuer Gleichgewichtsposition zur Ruhe kommt. Diese Beschreibung ist gerechtfertigt, wenn der Huang-Rhys-Faktor sehr groß ist $S \gg 1$ und gleichzeitig die Temperatur sehr niedrig ist, so dass nie genügend Phononen angeregt sind, um die Konfiguration zu verändern. Allerdings wurden von mehreren Gruppen [37, 38] Huang-Rhys-Faktoren in der Nähe von 1 gefunden, was diese Beschreibung zweifelhaft macht. Im dynamischen Fall des Jahn-TellerEffektes kann das System von einer äquivalenten Konfiguration in die andere tunneln. Zur Beschreibung verwendet man eine Superposition der verschiedenen Grundzustände.

Marcet et al. [37] haben gezeigt, dass für eine verbesserte Beschreibung ihrer experimentellen Daten das dynamische Regime angenommen werden muss. Dazu haben sie sowohl den Grundzustand ${ }^{5} T_{2}$ als auch den angeregten Zustand ${ }^{5} E$ mit einer $E$-Mode gekoppelt und damit eine konsistente Beschreibung erhalten. Der Jahn-Teller Effekt wirkt sich jetzt nicht mehr durch Aufhebung von Entartungen aus, sondern im Wesentlichen nur noch durch seinen Ham-Faktor. Dieser spielt überall dort eine Rolle, wo der Bahndrehimpuls beiträgt, also bei der Spin-Bahn-Wechselwirkung und beim Zeeman-Term (siehe Kapitel 2.2.3).

\subsubsection{Optische Übergänge und Magneto-Optik}

Optische Übergänge Zwischen verschiedenen elektronischen Zuständen eines Atoms können Übergänge stattfinden, wenn es eine entsprechende Wechselwirkung $\hat{V}$ gibt, die diese beiden Zustände vermischen kann. Das bedeutet nach Fermis goldener Regel nichts anderes, als dass die Übergangswahrscheinlichkeit $P$ abhängig ist vom Matrixelement dieser Wechselwirkung mit den beiden Zuständen:

$$
P \propto|\langle f|\hat{V}| i\rangle|^{2}
$$

Dabei steht $i$ für den Ausgangszustand und $f$ für den Endzustand. Bei optischen Übergängen ist typischerweise der elektrische Dipol-Operator ausschlaggebend für den Übergang 
so dass

$$
\hat{V}=\sum_{i} e \vec{r}_{i} \cdot \vec{\epsilon}_{E}
$$

wobei $\vec{\epsilon}_{E}$ der Einheitsvektor der Polarisation parallel zum $\vec{E}$-Feld der Strahlung ist. In manchen Fällen spielen auch magnetische Dipol- und elektrische Quadrupol-Strahlung eine Rolle, allerdings haben sie um viele Größenordnungen kleinere Übergangswahrscheinlichkeiten.

Da der elektrische Dipol-Operator ungerade Parität besitzt, erkennt man sofort eine Auswahlregel für optische Übergänge: Nur Übergänge zwischen Zuständen verschiedener Parität sind erlaubt, da ansonsten das Matrixelement (Gleichung 2.21) verschwindet. Dadurch sind zum Beispiel optische Übergänge eines Elektrons zwischen zwei $d$-Orbitalen (die beide gerade Parität besitzen) elektrisch-dipol-verboten. An dieser Stelle spielt das Kristallfeld jetzt eine entscheidende Rolle: Wenn es Terme ungerader Parität besitzt, so werden die $d$-Zustände im Kristallfeld mit anderen Atomzuständen ungerader Parität gemischt. Diese Beimischung hat praktisch keine Auswirkungen auf die Energieniveaus, wie sie im Laufe dieses Kapitels betrachtet wurden. Allerdings werden die Übergänge zwischen verschiedenen $d$-Orbitalen im Kristallfeld jetzt auch in der elektrischen DipolWechselwirkung schwach erlaubt. Wie in Gleichung $2.19 \mathrm{zu}$ sehen war, enthält schon das Tetraederfeld (im Gegensatz zum Oktaederfeld) einen Term ungerader Parität. Außerdem sind durch trigonale und tetragonale Verzerrungen weitere Beimischungen vorhanden. Typischerweise bleibt damit der elektrische Dipol-Übergang um mehrere Größenordnungen stärker als Quadrupol- oder magnetische Übergänge [25].

Die so genannten intra-3d-Übergänge zwischen verschiedenen Kristallfeldzuständen liegen energetisch gesehen im sichtbaren bis infraroten Spektralbereich. Wie in Kapitel 2.2.1 abgeleitet, haben oktaedrische Strukturen dabei höhere Energien und liegen meist im Sichtbaren, während bei tetraedrischer Koordination auf Grund der kleineren Aufspaltung die meisten Übergänge im Infraroten liegen. Die Farbigkeit vieler chemischer Komplexe in wässriger Lösung ist genau mit diesen intra-3d-Übergängen verknüpft, meist liegen hier oktaedrische Komplexe vor.

Franck-Condon-Prinzip Es wurde bereits in Abbildung 2.13 gezeigt, wie elektronische Zustände das Gitter auf Grund des Jahn-Teller-Effektes verzerren können. Verschiedene Zustände verursachen dabei verschiedene Verzerrungen, was sich natürlich auf die optischen Übergänge zwischen diesen elektronischen Zuständen auswirkt. Meist wird zur Beschreibung die Franck-Condon-Näherung benutzt. Dabei wird angenommen, dass die elektronischen Wechselwirkungen und damit der optische Übergang wesentlich schneller ablaufen als die Bewegung der Atomrümpfe.

Befindet sich also ein Atom im Grundzustand $a$, wie in Abbildung 2.14 gezeigt, so muss für einen Übergang in den Zustand $b$ das absorbierte Photon eine Energie besitzen, die größer ist als die Differenz der elektronischen Zustände in ihrem relaxierten Zustand. Denn bezüglich der so genannten Konfigurations-Koordinate $Q$ ist $b$ nicht relaxiert, wenn das System vom vibronischen Grundzustand von $a$ startet. Nach dem Franck-Condon-Prinzip entspricht ein optischer Übergang einer senkrechten Linie im Konfigurations-Koordinaten- 


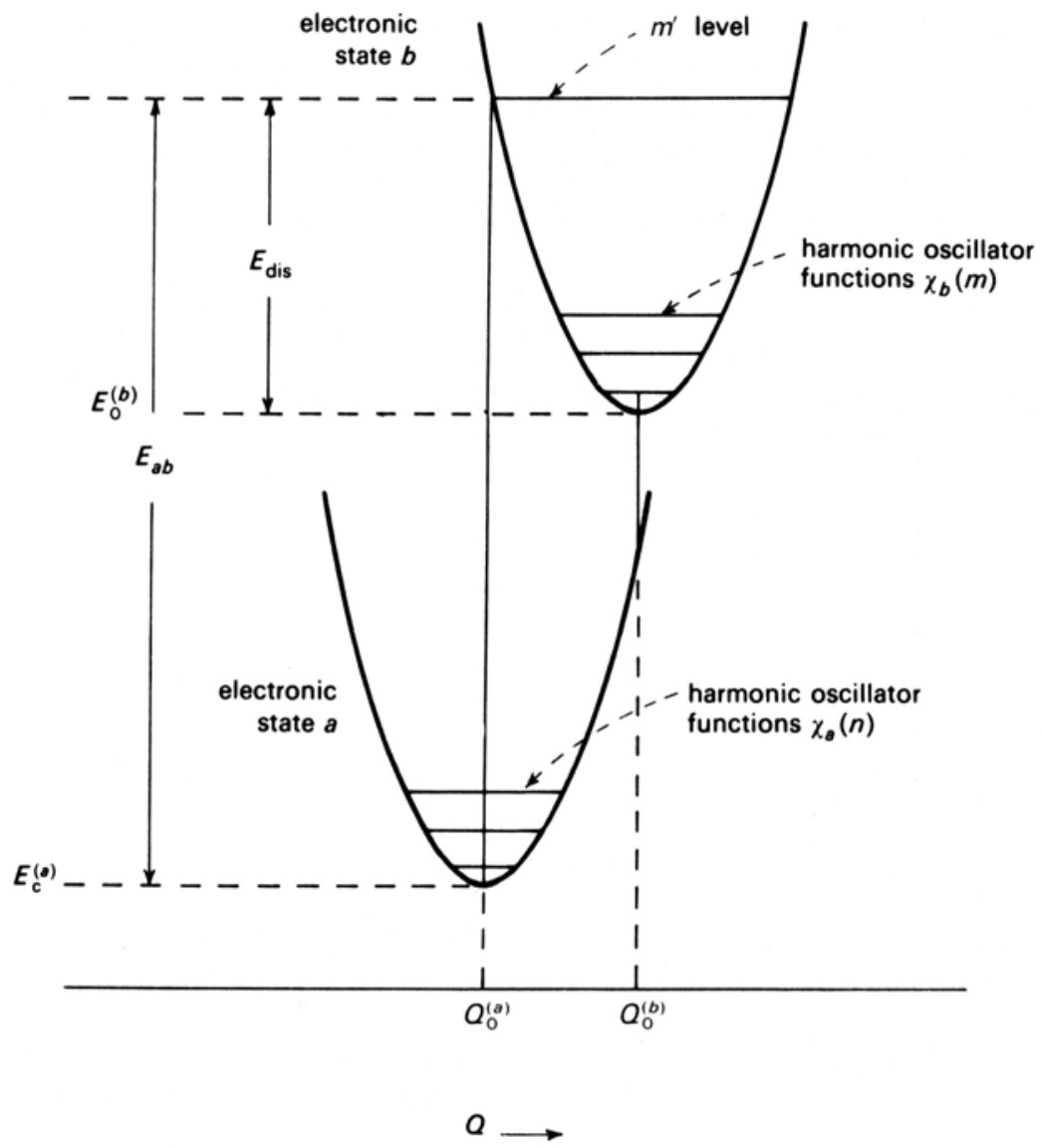

Abbildung 2.14: Konfigurations-Koordinaten-Diagramm für zwei elektronische Zustände in harmonischer Näherung nach [26]. Die Parabeln geben die quantisierten vibronischen Zustände der jeweiligen elektronischen Konfigurationen an. Obwohl deren Grundzustände nur um $\Delta E=E_{0}^{(b)}-E_{0}^{(a)}$ auseinander liegen, ist das Maximum der Absorption bei $E_{a b}$.

Diagramm, so dass die Absorption in einen vibronisch angeregten Zustand von $b$ erfolgt. Dies kann auch semiklassisch verstanden werden, wenn man die vibronischen Zustände durch die Eigenfunktionen eines harmonischen Oszillators nähert. Näherungsweise hängt das Matrixelement für die Übergangswahrscheinlichkeit jetzt vom Überlapp dieser Oszillator-Wellenfunktionen ab. Geht man von tiefen Temperaturen und damit vom vibronischen Grundzustand bei $a$ aus, muss jetzt die Eigenfunktion von $b$ gefunden werden, die den größten Überlapp hat. Die größte Aufenthaltswahrscheinlichkeit von Zuständen im harmonischen Oszillator ist genau in der Nähe der klassischen „Wendepunkte“ einer Schwingung, also am Schnittpunkt der Energie mit der Parabel des Potentials. Damit wird auch hier die größte Übergangswahrscheinlichkeit für einen möglichst senkrechten Übergang erwartet.

Magneto-Optik Wenn ein Material einem Magnetfeld ausgesetzt wird, so tritt der so genannte Zeeman-Effekt auf. Dies äußert sich in einem zusätzlichen Term im HamiltonOperator, der vom Magnetfeld abhängt: 


$$
\hat{H}_{Z}=\mu_{B}(\vec{L}+2 \vec{S}) \vec{B}
$$

Dabei ist $\mu_{B}$ das Bohr'sche Magneton und $\vec{B}$ das Magnetfeld. Der $g$-Faktor wurde als 2 angenommen. Allerdings ist der Bahndrehimpuls-Anteil durch den Ham-Faktor reduziert, so dass sich ein effektiver Hamilton-Operator $\hat{H}_{Z}$ eff ergibt:

$$
\hat{H}_{Z \text { eff }}=\mu_{B}(\kappa \vec{L}+2 \vec{S}) \vec{B}
$$

Durch die Spin-Bahn-Wechselwirkung ist $m_{S}$ im Nullfeld keine gute Quantenzahl. Bei hohen Feldern (mehrere Tesla) richten sich die Spins allerdings entlang des Magnetfeldes aus und die Energieniveaus zeigen ein lineares Verhalten mit $\vec{B}$. Dies ist, zusammen mit den Aufspaltungen im Modell von Marcet et al. [37], in Abbildung 2.15 gezeigt.

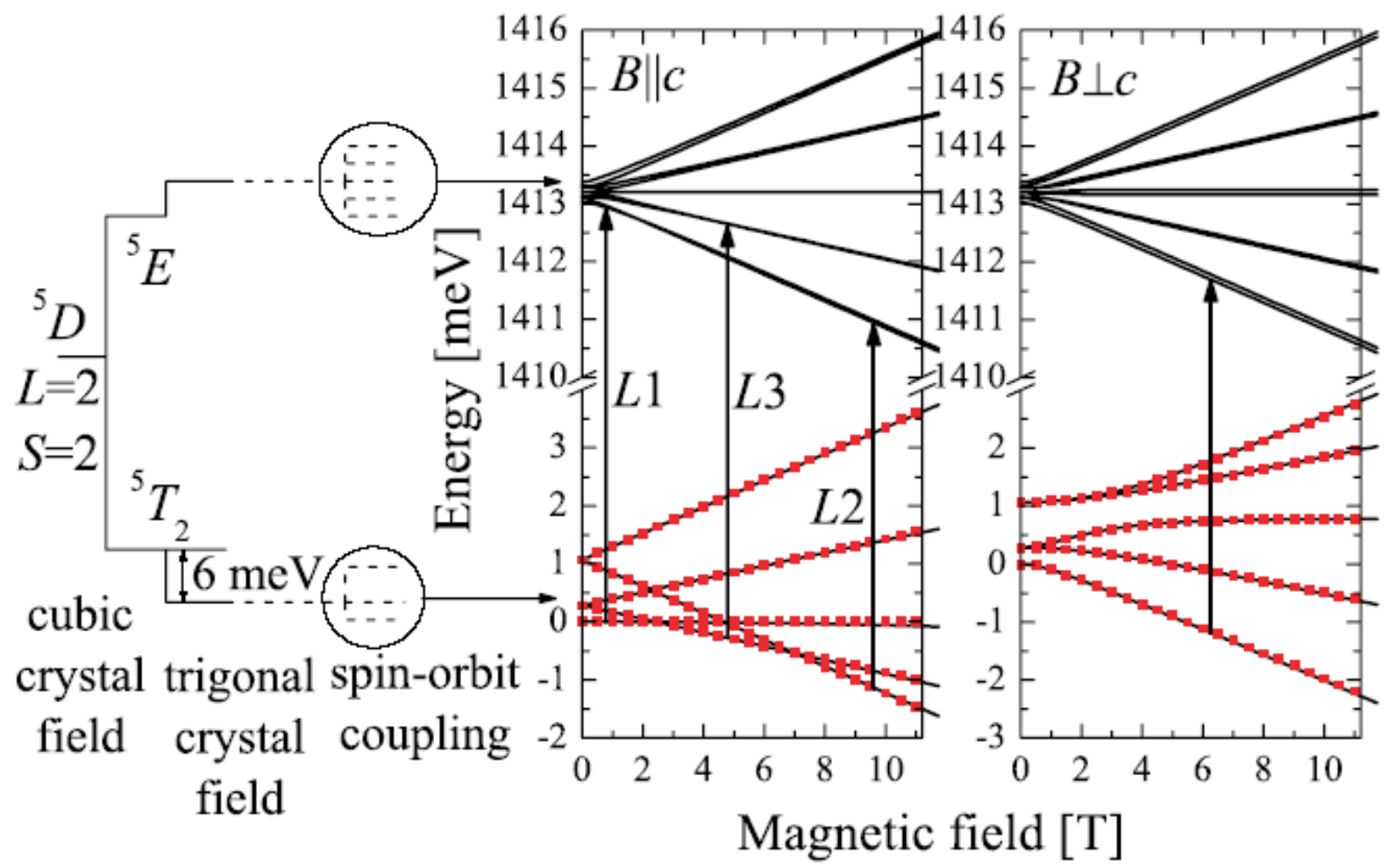

Abbildung 2.15: Energieniveaus von $\mathrm{Mn}^{3+}$ in GaN nach [37]. Die $\vec{B}$-Feld-Abhängigkeit in Faradayund Voigt-Konfiguration ist rechts dargestellt. 


\subsection{Verdünnte magnetische Halbleiter (DMS)}

Werden in einem Halbleiter ein Teil der Atome durch magnetische Ionen ersetzt, so nennt man ihn „verdünnten magnetischen Halbleiter“ (siehe Abbildung 2.16), kurz DMS (nach „diluted magnetic semiconductor"). Dieser Materialtyp wurde vor etwa dreißig Jahren entdeckt [39] und muss von den magnetischen Halbleitern klar unterschieden werden, die in „undotierter" Form bereits magnetisch sind, aber typischerweise auf Grund komplizierter Bandstrukturen und ungünstiger physikalischer Eigenschaften nicht so interessant für die angewandte Forschung sind [40]. Mit Hilfe der DMS könnte es möglich sein, gleichzeitig Spin und Ladung der Elektronen für Informationsspeicherung und -verarbeitung einzusetzen. Neuartige Bauteile wie Spin-Transistoren könnten durch DMS ebenso realisiert werden wie Quantencomputer.

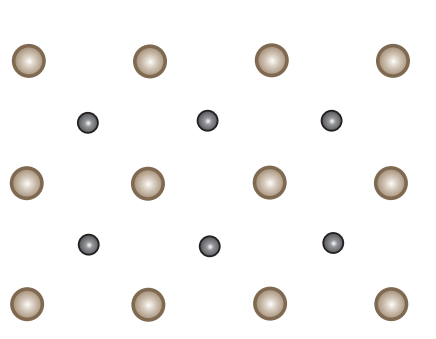

Halbleiter (intrinsisch)

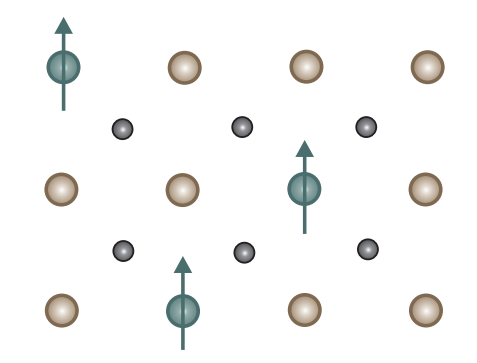

$\mathrm{D}_{\text {iluted }} \mathrm{M}_{\text {agnetic }} \mathrm{S}_{\text {emiconductor }}$

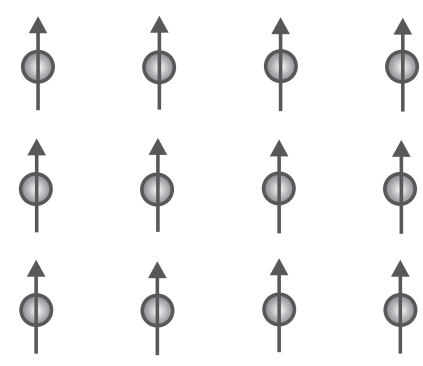

Ferromagnet

Abbildung 2.16: Struktur eines verdünnten magnetischen Halbleiters im Vergleich zu intrinsischem Halbleiter und klassischem Ferromagneten wie etwa Eisen.

Als Ausgangsmaterial zur Herstellung von DMS werden auch klassische Gruppe IV-Halbleiter wie Silizium und Germanium verwendet, vor allem aber III-Vund II-VI-Halbleiter. Diese kristallisieren typischerweise in Zinkblende- oder WurtzitKristallstrukturen und können auf Si-Substraten epitaktisch gewachsen werde, so dass eine Integration in die herkömmliche Si-Technologie möglich ist. In diese Matrix werden nun auf dem Kation-Gitterplatz Übergangsmetalle oder Seltene Erden eingebaut, die teilweise gefüllte $d$ - bzw. $f$-Schalen aufweisen. Die Elektronen in diesen teilweise gefüllten Schalen ordnen sich auf Grund der Hundschen Regeln so an, dass maximaler Gesamtspin erreicht wird. So hat beispielsweise $\mathrm{Mn}^{3+}$ mit seiner $d^{4}$-Konfiguration einen Gesamtspin von $S=2$ und $\mathrm{Gd}^{3+}$ mit seinen sieben $f$-Elektronen $S=7 / 2$. Diese lokalisierten Spins zeigen nun unterschiedliche Wechselwirkungen untereinander und mit der umgebenden Matrix, was zu verschiedenen magnetischen Kopplungen zwischen den magnetischen Ionen führen kann. Bei manchen dominieren die antiferromagnetischen Wechselwirkungen und bei manchen die ferromagnetischen, je nach Matrix und Typ der magnetischen Verunreinigung.

\subsubsection{Magnetische Kopplung}

Bei allen Betrachtungen der magnetischen Kopplungen muss man sich vom Modell des isolierten (magnetischen) Ions lösen, wie es im Kapitel 2.2 für die Kristallfeldtheorie einge- 
führt wurde. Es ist jetzt sinnvoll, die Ein-Elektronenzustände als Störstellenbänder in der Bandstruktur des Halbleiters, der als Matrix benutzt wird, zu betrachten [41, 42]. Nach wie vor relevant ist dabei die Aufspaltung zwischen den $e$ - und $t_{2}$-Niveaus und deren jeweilige Besetzung.

Superaustausch und Doppelaustausch Der Superaustausch beschreibt eine Wechselwirkung zwischen den lokalisierten $d$-Orbitalen der Verunreinigungen, der zu antiferromagnetischer Kopplung führt. Wenn die magnetischen Atome (bei genügend hoher Konzentration) nahe beieinander im Gitter liegen, so hybridisieren die $d$-Zustände und bilden „bindende" und „antibindende“ Hybridorbitale. Dabei können allerdings nur jeweils Zustände gleicher Spinrichtung miteinander hybridisieren. Es ergibt sich ein Energiegewinn aus der antiferromagnetischen Anordnung, sobald die $d$-Zustände teilweise besetzt sind. Schematisch ist diese Wechselwirkung in Abbildung 2.17 zusammengefasst. Teilweise wird die Hybridisierung auch als virtuelles ,hopping“ zwischen den beteiligten Niveaus beschrieben.

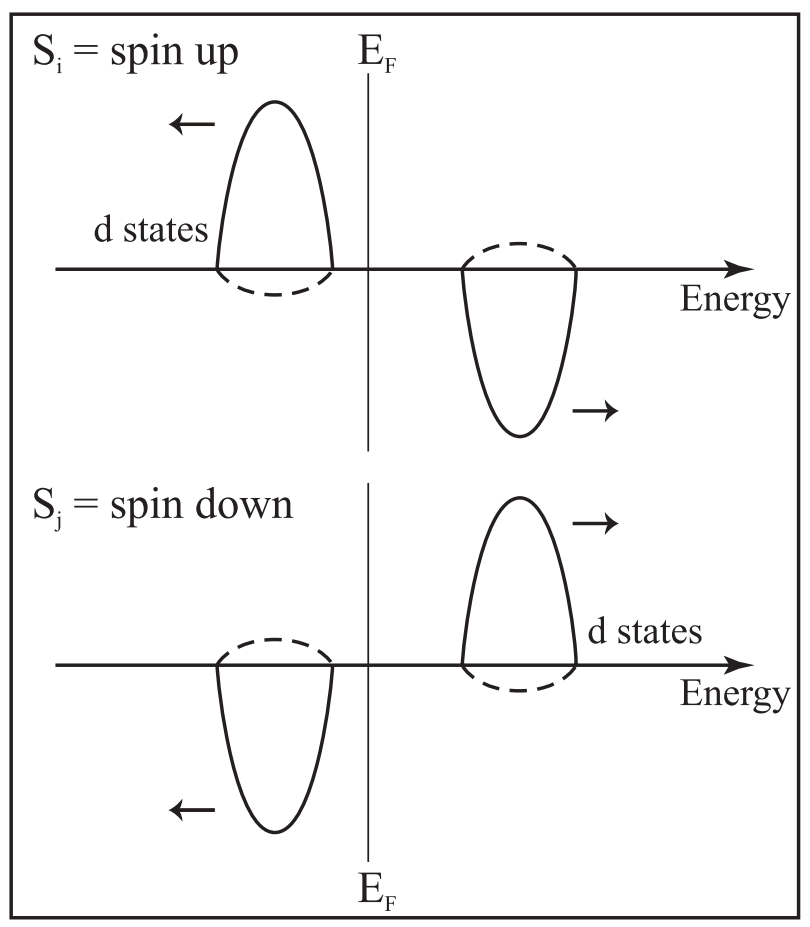

Abbildung 2.17: Superaustausch: Die $d$-Zustände zweier Mn-Atome $i$ und $j$ mit verschiedenen Spinrichtungen hybridisieren. Dabei wird jeweils das unten liegende Niveau abgesenkt und das obere angehoben, so dass sich ein Energiegewinn ergibt, falls die Fermienergie innerhalb oder zwischen den Bändern liegt. Nach [43].

Mit dem gleichen Modell kann man auch den Doppelaustausch beschreiben (siehe Abbildung 2.18), der zu einer ferromagnetischen Kopplung beiträgt. Wenn bei ferromagnetischer Anordnung, also bei gleichen Spinausrichtungen der Verunreinigungen, Hybridisierung auftritt, äußert sich dies in einer Verbreiterung der beteiligten Niveaus. Sind diese Niveaus teilweise besetzt, kann das System durch diese Hybridisierung seine Energie mini- 
mieren und der ferromagnetische Zustand wird bevorzugt. Auch diese Hybridisierung wird manchmal als virtuelles ,hopping“" beschrieben. Tritt das magnetische Ion in gemischter Valenz auf, beispielsweise $\mathrm{Mn}^{2+}$ und $\mathrm{Mn}^{3+}$, dann kann ein Elektron vom $\mathrm{Mn}^{2+}$ auch ein reales „hopping“ zum $\mathrm{Mn}^{3+}$ ausführen. Dadurch wird dieser Mechanismus noch einmal wesentlich verstärkt.

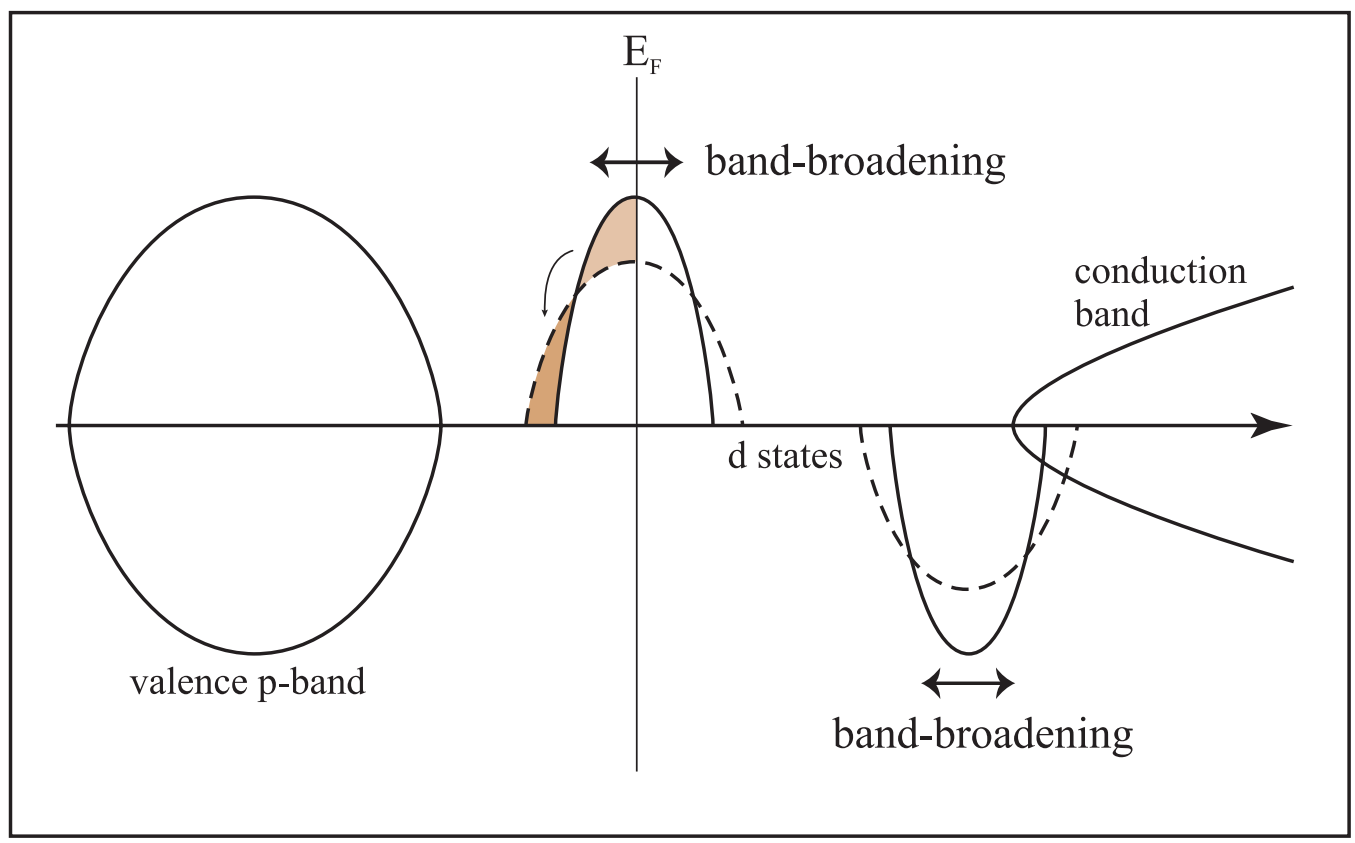

Abbildung 2.18: Doppelaustausch: Wenn alle Mn-Atome ferromagnetisch ausgerichtet sind, verbreitern sich die $d$-Bänder. Liegt das Fermi-Niveau innerhalb eines solchen Bandes, wird dadurch ein Energiegewinn erreicht. Nach [43].

Superaustausch und Doppelaustausch müssen immer gleichzeitig betrachtet werden, da sie auf den gleichen Mechanismen beruhen. Der Superaustausch liefert dabei immer einen antiferromagnetischen Beitrag, da bei allen Übergangsmetallen die $d$-Niveaus teilweise besetzt sind. Der Doppelaustausch kann aber nur einen ferromagnetischen Beitrag liefern, wenn das Ferminiveau innerhalb eines Bandes liegt, also entweder $e$ - oder $t_{2}$-Niveau teilweise besetzt sind. Dies ist im Tetraederfeld nur bei den Konfigurationen $d^{1}, d^{3}, d^{4}$, $d^{6}, d^{8}$ und $d^{9}$ der Fall. Es kann gezeigt werden, dass der Energiegewinn durch Superaustausch proportional zur Konzentration $x$ der magnetischen Verunreinigung ist, während der durch Doppelaustausch mit $\sqrt{x}$ skaliert [43]. Sobald also Doppelaustausch möglich ist, wird er über den Superaustausch dominieren und für eine ferromagnetische Kopplung sorgen.

$s, p$ - $d, f$-Austausch und RKKY Andere Mechanismen zur magnetischen Kopplung benötigen freie Ladungsträger in Bandzuständen der Halbleitermatrix. Dabei treten die lokalen $d$ - oder $f$-Zustände in Wechselwirkung mit den Bändern des Halbleiters, die $s$ Charakter haben im Falle des Leitungsbandes und p-Charakter im Falle des Valenzbandes. Diese Wechselwirkung beziehungsweise Hybridisierung ist um so stärker, je näher die 
Band- und lokalen Zustände energetisch liegen. So ergibt sich beispielsweise auf Grund der $s$ - $d$-Hybridisierung ein Austauschterm der Form

$$
\hat{H}_{e x}=-x N_{0} \alpha\left\langle S_{z}\right\rangle s_{z}
$$

wobei $x N_{0}$ die Konzentration der magnetischen Ionen ist, $\left\langle S_{z}\right\rangle$ deren mittlerer Spin und $s_{z}$ die Spinkomponente der freien Elektronen entlang des magnetischen Feldes. $\alpha$ ist die Kopplungskonstante dieses $s$ - $d$-Austauschs. Entsprechend kann man eine Kopplung mit den Löchern des Valenzbandes definieren, deren Kopplungskonstante mit $\beta$ bezeichnet wird.

Der RKKY-Mechanismus (nach Ruderman, Kittel, Kasuya und Yoshida) führt zu einer oszillatorischen Abhängigkeit der Kopplungskonstanten zwischen den magnetischen Ionen von deren Abstand. Für nächste Nachbarn ergibt sich eine ferromagnetische Kopplung, die mit zunehmendem Abstand irgendwann auf antiferromagnetisch umschlägt. Für große Abstände $r$ ergibt sich eine Abhängigkeit proportional zu $\cos r / r^{3}$, die Kopplung wird also immer schwächer. Dieses Modell wurde ursprünglich für Metalle entwickelt und benötigt sehr hohe Ladungsträgerkonzentrationen.

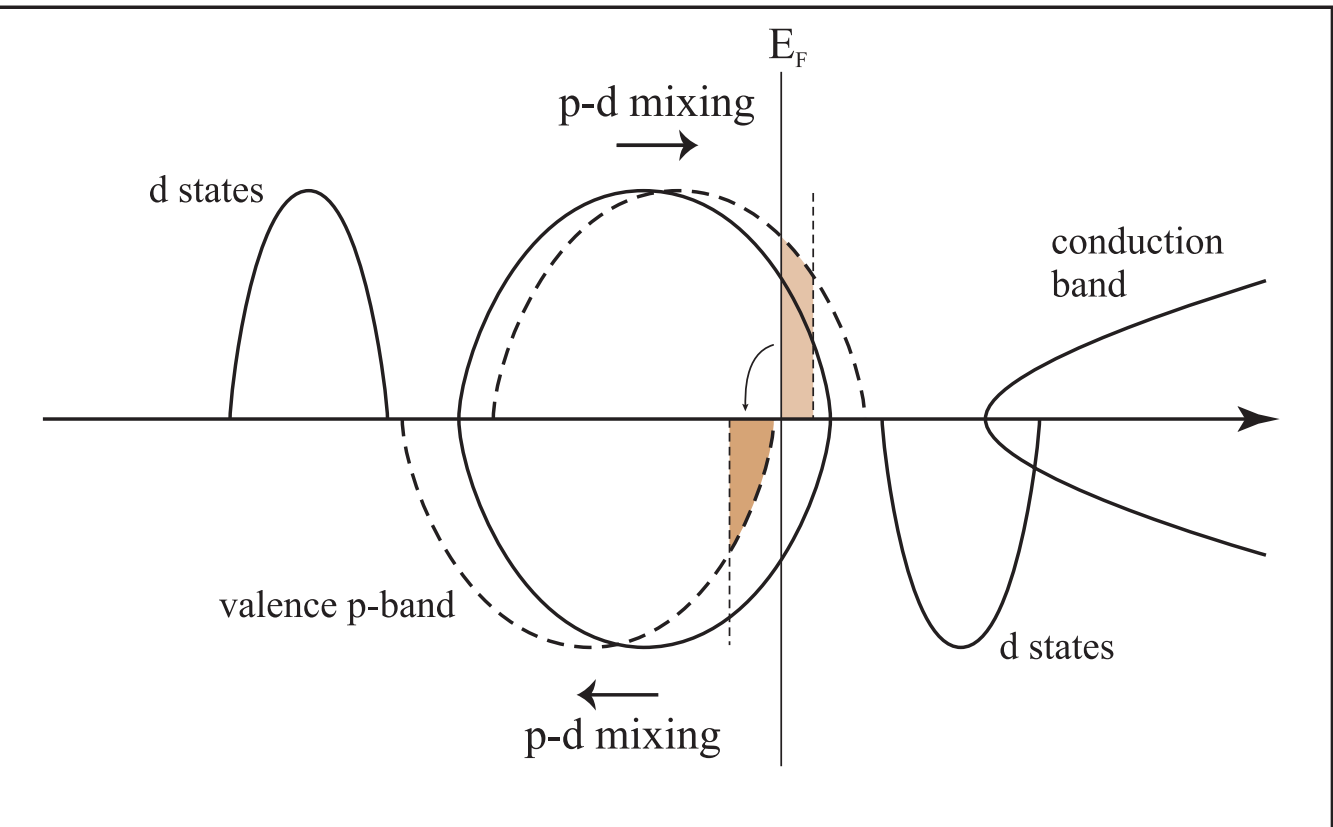

Abbildung 2.19: Durch $p$ - $d$-Austausch hybridisieren Valenzband und die $d$-Zustände. Liegen die $d$ Niveaus nahe genug der Valenzbandkante, so können bei geeigneter Dotierung spin-polarisierte Löcher entstehen, die die Wechselwirkung vermitteln. Nach [43].

Abbildung 2.19 zeigt schematisch einen $p$-d-Austausch. Sind durch geeignete Wahl des Übergangsmetalls oder zusätzliche Dotierung freie Löcher im System vorhanden, werden diese polarisiert und vermitteln eine ferromagnetische Wechselwirkung zwischen den magnetischen Ionen. Dieser Mechanismus ist im Gegensatz zu Super- und Doppelaustausch auch dann wichtig, wenn die $d$-Niveaus unterhalb der Valenzbandkante liegen [44]. 


\subsubsection{DMS auf GaN-Basis}

Die ersten untersuchten DMS waren meist II-VI-Halbleiter. Allerdings wurden nur sehr niedrige Übergangstemperaturen von einigen Kelvin erreicht. Erst bei dem III-VHalbleiter GaAs mit Mn wurde ein wesentlicher Sprung erreicht, plötzlich waren CurieTemperaturen über $100 K$ möglich und das Forschungsgebiet der DMS erlebte einen Boom. Ferromagnetismus bei Raumtemperatur war in greifbare Nähe gerückt. Im Jahr 2000 schließlich publizierten Dietl et al. [9] eine Theorie, nach der mit GaMnN unter bestimmten Vorraussetzungen dieses Ziel erreicht werden könnte. Vorrausgesetzt wurde dabei eine Mn-Konzentration von $5 \%$ und eine Konzentration freier Löcher von $p=3.5 \times 10^{20} \mathrm{~cm}^{-3}$. Die vorrausgesagten Curie-Temperaturen sind in Abbildung 2.20 zu sehen, außer für GaN wurde für dessen II-VIer Äquivalent ZnO RaumtemperaturFerromagnetismus vorhergesagt.

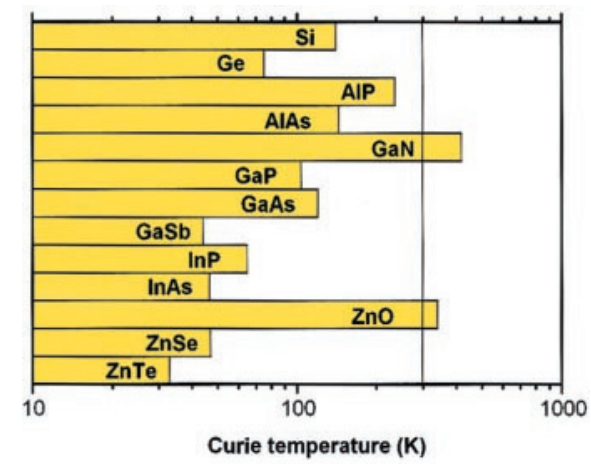

Abbildung 2.20: Von Dietl et al. [9] berechnete Curie-Temperaturen für verschiedene Halbleiter mit $5 \%$ Mn-Gehalt und einer Lochkonzentration von $p=3.5 \times 10^{20} \mathrm{~cm}^{-3}$.

Die zugrunde liegende Theorie benutzt ein Zener-Modell [45] mit der RKKYWechselwirkung. Dieses Zener-Modell berücksichtigt im Gegensatz zur reinen RKKYTheorie die Anisotropie der Wechselwirkung, die durch die Spin-Bahn-Wechselwirkung verursacht wird. Damit können experimentelle Befunde bei GaMnAs und ZnMnTe:N erklärt werden wie etwa deren Curie-Temperaturen und die beobachtete Abhängigkeit der Magnetisierung von Verspannungen [46].

Als Folge dieser Veröffentlichung wurden weitreichende experimentelle Untersuchungen stimuliert und verschiedene theoretische Ansätze unternommen, um das System GaMnN genauer zu untersuchen. Denn ob der gleiche Ansatz, der den Ferromagnetismus bei GaMnAs erklären konnte, einfach auf GaMnN übertragbar ist, musste erst überprüft werden. So haben beispielsweise Mahadevan und Zunger auf der Grundlage von DFT Rechnungen [47] ein Modell für die $d$-Niveaus in verschiedenen III-V-Halbleitern aufgestellt, das hieran Zweifel weckt. Die wesentlichen Ergebnisse für GaMnAs und GaMnN sind in Abbildung 2.21 dargestellt. Die $t_{2}$-Niveaus des Mn hybridisieren mit den Valenzbandzuständen der Matrix. Im Fall von GaAs sind die ursprünglichen $t_{2}$-Niveaus dabei weit unterhalb der Valenzbandkante angesiedelt, so dass sich das Loch in einem valenzbandartigen Zustand befindet (dangling-bond Hybrid), der delokalisiert ist. Das Valenzband von GaN befindet sich deutlich unterhalb der $t_{2}$-Zustände des $\mathrm{Mn}$, so dass nach 
Hybridisierung sich das Loch in einem Zustand befindet, der am Mn-Atom lokalisiert ist (Kristallfeld-Resonanz).

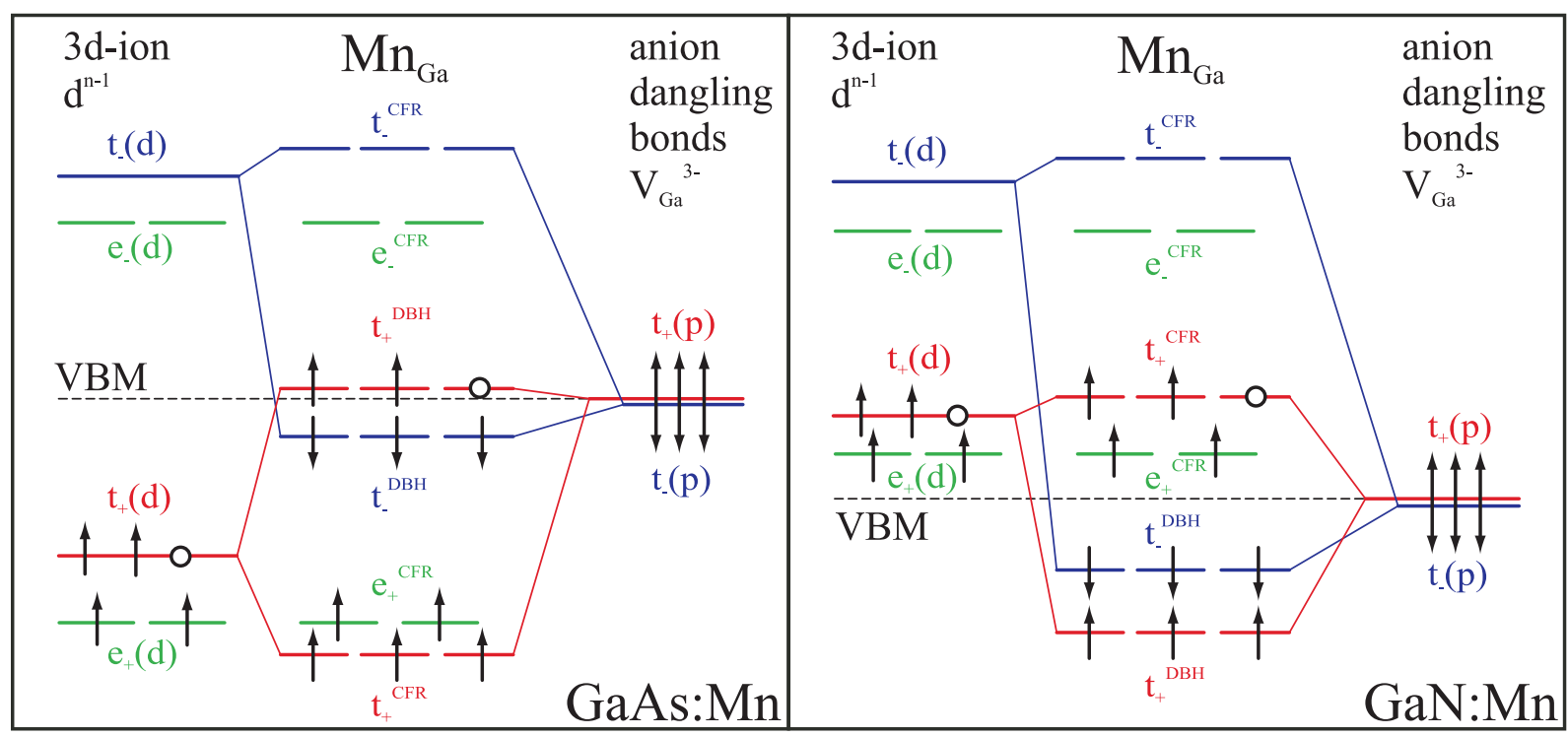

Abbildung 2.21: Schematisches Modell der elektronischen Zustände von Mn in GaAs (links) und GaN (rechts) nach [47]. Jeweils an der linken Seite ist das freie $\mathrm{Mn}^{3+}$-Ion mit seinen durch AustauschWechselwirkung und Kristallfeld aufgespaltenen $d$-Niveaus dargestellt, an der rechten Seite die ,dangling bonds" der Anionen. Durch Hybridisierung der $t_{2}$-Niveaus ergeben sich die in der Mitte gezeigten Kristallfeld-Resonanzen (CFR) und dangling-bond Hybride (DBH).

Ein wesentliches Problem der meisten Theorien besteht in der Behandlung der Legierungsunordnung bei verdünnten magnetischen Halbleitern. In einem mean-field-Ansatz wird einfach darüber gemittelt und nur die Konzentration als Parameter betrachtet. Sato et al. [14] konnten mit Hilfe eines Monte-Carlo-Verfahrens zeigen, dass dabei die berechneten Curie-Temperaturen wesentlich überschätzt werden. Besonders stark ist dieser Effekt bei niedrigen Konzentrationen und Wechselwirkungen mit geringer Reichweite wie dem Doppelaustausch. Zurück geführt wird er auf das Perkolationsproblem, denn um einen Ferromagneten zu erhalten, muss die Kopplung auf makroskopischen Skalen aufrecht erhalten werden. Aber erst bei Konzentrationen von etwa $20 \%$ erreichen die mit der Monte-Carlo-Methode berechneten $T_{C}$ den Wert, der aus der mean-field-Näherung folgt. Diese Erkenntnis hatte nur geringe Auswirkungen auf Systeme wie GaMnAs, bei denen der Magnetismus durch eine relativ langreichweitige $p$ - $d$-Wechselwirkung vermittelt wird. Bei GaMnN, wo der kurzreichweitige Doppelaustausch den Hauptbeitrag zum Ferromagnetismus liefert, sinken die erreichbaren Curie-Temperaturen plötzlich auf deutlich unter $100 K$. Damit wäre dieses Materialsystem gänzlich ungeeignet für Anwendungen bei Raumtemperatur.

Das Problem der Perkolation wurde in [48] noch genauer untersucht und es stellte sich heraus, dass GaMnN instabil gegenüber spinodaler Entmischung ist. Das bedeutet, dass bei hohen Temperaturen, wie sie beim Wachstum herrschen, die Mn-Atome durch eine attraktive Wechselwirkung benachbarte Gitterpositionen bevorzugen und daher auf atomarer Skala Bereiche erhöhter und erniedrigter Konzentration bilden. Diese Entmischung 
führt zu einer weiteren Erniedrigung der Curie-Temperaturen unterhalb der Perkolationsgrenze, aber zu einer Erhöhung oberhalb dieser Schwelle. Dies kann man dadurch verstehen, dass durch die Entmischung die Konzentration lokal deutlich erhöht ist. Unterhalb der Perkolation sind aber die Abstände zwischen diesen Bereichen noch einmal deutlich erhöht im Vergleich zur zufälligen Anordnung, was den Doppelaustausch weiter abschwächt. Oberhalb der Perkolation sind die Bereiche hoher Konzentration verknüpft und man erhält effektiv einen DMS mit eben dieser erhöhten Konzentration und daraus folgenden geringeren Mn-Mn-Abständen. Ab den kritischen $20 \%$ Mn-Gehalt wurden damit wieder Curie-Temperaturen oberhalb Raumtemperatur vorhergesagt. Durch Berücksichtigung des Wachstumsprozesses wurde von Fukushima et al. [49] berechnet, dass sich die Entmischungen Fadenartig in Wachstumsrichtung ausbilden. Durch DeltaDotierung könnte eine Kopplung der einzelnen Fäden miteinander erreicht werden und Raumtemperatur-Ferromagnetismus auch bei Konzentrationen von $5 \%$ ermöglicht werden.

Nachdem GaN als potentielles Basismaterial für einen DMS identifiziert worden war, wurden auch andere magnetische Ionen außer Mn untersucht. Vorreiter waren hierbei Sato et al. [13], die verschiedene Übergangsmetalle in mehreren II-VI- und III-V-Halbleitern betrachteten. Für GaN wurde dabei mittels einer mean-field Theorie Ferromagnetismus bei Dotierung mit V, Cr und Mn vorhergesagt, während Dotierung mit Fe, Co oder Ni zu Spin-Glas-Verhalten führt (siehe Abbildung 2.22).

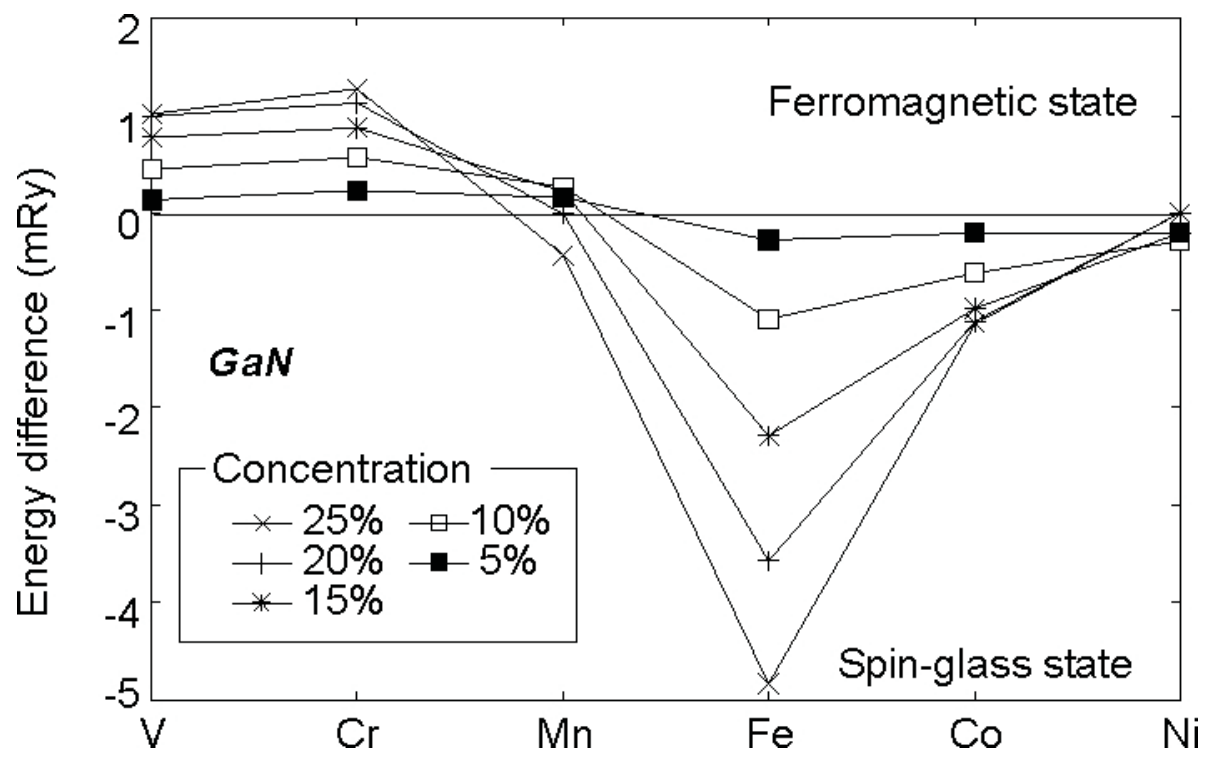

Abbildung 2.22: Stabilität der ferromagnetischen gegenüber der antiferromagnetischen (Spin-Glas)Phase bei GaN dotiert mit verschiedenen Übergangsmetallen nach [13].

Außer Übergangsmetallen mit ihren $d$-Elektronen können die Seltenen Erden mit den teilweise gefüllten $f$-Schalen für lokale magnetische Momente sorgen. Da die $4 f$-Orbitale durch die örtlich weiter außen liegenden, vollbesetzten $5 p$-Orbitale sehr effektiv von der Umgebung abgeschirmt werden, ist nur eine schwache Wechselwirkung mit der Matrix zu erwarten. Dies wurde so auch von Svane et al. [50] berechnet, die auf Grund dessen 
keine Erklärung für Raumtemperatur-Ferromagnetismus finden, wie er experimentell bei GaGdN beobachtet wurde [51, 52]. Ein möglicher Ansatz findet sich bei Dalpian et al. [15], die eine ferromagnetische Kopplung voraussagen, die durch Elektronen vermittelt wird. Auf Grund einer $s$ - $f$-Kopplung werden die Leitungsbandelektronen durch die GdIonen polarisiert und wirken als Träger des Ferromagnetismus. Diese Polarisierung könnte auch erklären, warum die experimentell gefundenen Werte eine Magnetisierung von über $1000 \mu_{B} / G d$ ergeben, obwohl jedes Gd-Ion nur sieben ungepaarte Elektronen und damit $7 \mu_{B}$ besitzt. 


\section{Wachstum von GaN und GaMnN}

Die im Rahmen dieser Arbeit untersuchten Proben wurden alle mit epitaktischen Wachstumsmethoden hergestellt. GaMnN und GaGdN wurden am IV. Physikalischen Institut der Universität Göttingen mittels Molekularstrahl-Epitaxie (kurz MBE, nach „molecular beam epitaxy") hergestellt, weshalb diese Methode detaillierter vorgestellt werden soll. Die GaCrN-Proben wurden im Rahmen einer Zusammenarbeit am Forschungszentrum Jülich mittels Metall-organischer Gasphasen-Epitaxie (kurz MOCVD, nach „metal-organic chemical vapor deposition") produziert. Viele grundlegende Parameter sind auch auf diese Methode übertragbar, nur wesentliche Abweichung sind extra vermerkt. Eine dritte häufig verwendete Methode ist die Hydrid Gasphasen-Epitaxie (kurz HVPE, nach ,hydride vapor phase epitaxy“). Ausführliche Referenzen über das Wachstum von GaN sind: MBE [53], MOCVD [54] und HVPE [55].

\subsection{Molekularstrahl-Epitaxie (MBE)}

Der grundlegende Aufbau eines MBE-Systems ist in Abbildung 3.1 dargestellt. Die Anlage besteht aus einer UHV-Kammer, in der Quellen für die Ausgangs- und Dotiermaterialien sowie ein heizbarer Probenhalter untergebracht sind.

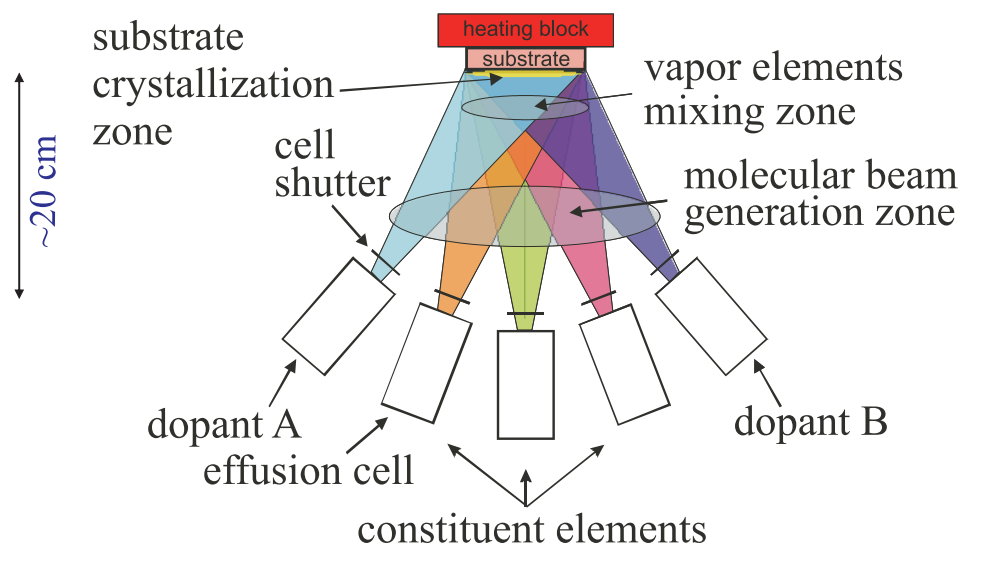

Abbildung 3.1: Aufbau einer MBE-UHV-Kammer

Im vorliegenden Fall wurde der Stickstoff durch eine $\mathrm{N}_{2}$-Plasmaquelle bereitgestellt, während die Metalle Ga, Al, Mn und Gd in Effusionszellen thermisch verdampft wurden. Der jeweilige Fluss des Molekularstrahls aus den Zellen kann durch ein Druckmessgerät, welches an die Position des Probenhalter gebracht wird, bestimmt werden. Man spricht dann vom ,beam equivalent pressure“ (BEP), der in der Größenordnung $10^{-7} \mathrm{mbar}$ für 
das Ga und $10^{-5}$ mbar für das $\mathrm{N}_{2}$ liegt. Die Dotieratome werden relativ zum Ga in entsprechend geringerer Dosis angeboten. Da die Abscheidung des Halbleiterkristalls bei etwa $10^{-5}$ mbar stattfindet, ist ein Hintergrunddruck von $10^{-11}$ mbar oder niedriger erstrebenswert, um Verunreinigungen des Materials unterhalb der ppm-Grenze (entsprechend etwa $\left.10^{17} \mathrm{~cm}^{-3}\right) \mathrm{zu}$ halten.

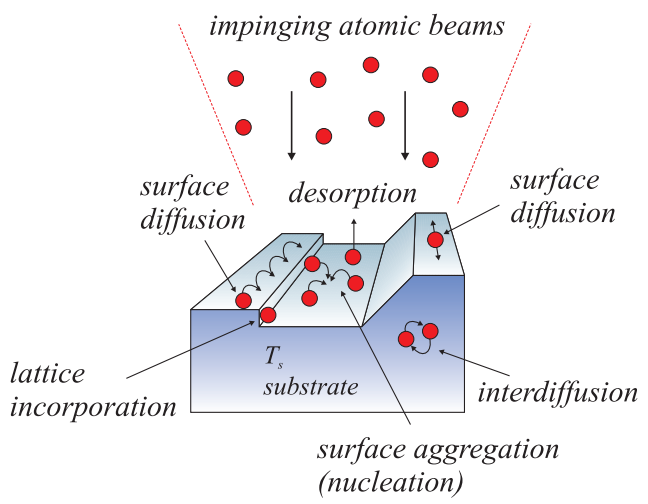

Abbildung 3.2: Oberflächenprozesse beim MBE-Wachstum

Das Grundprinzip der MBE ist in Abbildung 3.2 dargestellt. Die Atome treffen auf der Oberfläche der wachsenden Probe auf und werden dort adsorbiert. Sie diffundieren dann frei auf der Oberfläche, wobei die Beweglichkeit von der Substrattemperatur und der Oberflächenstruktur abhängt. Schließlich werden sie entweder desorbieren oder fest an die Oberfläche gebunden werden, bevorzugt an Stufen oder ähnlichen Defekten. Diese gebundenen Atome tragen zum weiteren Schichtwachstum bei.

Bei MBE ist, anders als bei MOCVD, keine chemische Reaktion an der Oberfläche nötig und auch die Ausgangsstoffe müssen nicht präpariert werden, sondern können einfach aus den Elementen verdampft werden. Daher ist es ein Verfahren, was extrem vielseitig ist. Das Wachstum findet dabei weit weg vom thermodynamischen Gleichgewicht statt. So können oftmals „Dotier“-Konzentrationen im Prozentbereich erreicht werden, sofern geeignete Parameter gewählt werden. Weiterhin können atomar glatte Oberflächen produziert werden, so dass diese Methode für das Wachstum vom Heterostrukturen ideal ist.

Die untersuchten Proben wurden mit zwei verschiedenen MBE-Systemen hergestellt. Die GaMnN-Proben auf Si-Substrat sowie die GaGdN-Proben wurden mit der Anlage „JUEL" gewachsen, die in [56] detaillierter beschrieben ist. Die Benennung dieser Proben ist „Jxxxx“, wobei „Xxxx“ für eine durchlaufende vierstellige Nummer steht. Die GaMnNProben auf Saphir-Substrat dagegen wurden mit der Anlage "GEN2" gewachsen, die in Göttingen neu aufgebaut wurde, wie in [57] beschrieben. Diese Proben werden mit „Gxxxx“ benannt.

\subsection{Parameter-Raum des MBE-Wachstums von GaN}

Die wichtigsten Parameter für das Wachstum sind die Substrattemperatur einerseits und die Zusammensetzung und Stärke des Molekularstrahls andererseits. Die Substrattempe- 
ratur kann direkt über eine Heizung kontrolliert werden und muss durch einen Regelkreis auf wenige Zehntel Grad konstant gehalten werden. Die Metall-Flüsse können durch die Zellentemperatur kontrolliert werden, je nach Substanz verdampft oder sublimiert diese näherungsweise nach $p \propto e^{\frac{\Delta H}{k T}}$. Dieser Druck ist dann direkt proportional zum entsprechenden Fluss auf die Probe. Die Stickstoff-Zelle wird durch den Stickstofffluss und die Radio-Leistung kontrolliert. Die Leistung sorgt für eine Aktivierung der $\mathrm{N}_{2}$-Moleküle durch Radiostrahlung und ändert damit das Verhältnis zwischen N-Ionen, N-Radikalen, aktivierten $\mathrm{N}_{2}$-Molekülen und nicht aktivierten $\mathrm{N}_{2}$-Molekülen. In den jeweiligen Anlagen wurde die $\mathrm{N}_{2}$-Quelle einmal optimiert und danach dieser Parameter konstant gehalten, um den Parameter-Raum einzuschränken.

Substrattemperatur Die Substrattemperatur ist entscheidend für die Oberflächendiffusion und Desorptionsrate der Atome auf der Probenoberfläche. Bei einer hohen Temperatur sind die Atome beweglicher. Dadurch finden sie leicht die optimalen Positionen zum Einbau und die Kristallqualität ist hoch. Bei niedrigen Temperaturen und entsprechend niedriger Beweglichkeit tritt bei GaN Insel- bzw. Stranski-Krastanov-Wachstum auf. Es bildet sich also kein glatter, hochqualitativer Film aus. Aus diesen Gründen sind hohe Wachstumstemperaturen erstrebenswert um einen homogenen Film mit hoher Kristallqualität zu erhalten. Bei GaN wird die Temperatur nach oben begrenzt durch die Desorption. Auch diese steigt mit der Temperatur, was sich zuerst in einer Verlangsamung der Wachstumsrate niederschlägt [58]. Ab etwa $850-900^{\circ} \mathrm{C}$ ist dieser Effekt allerdings so stark, dass eine GaN-Probe schneller sublimiert als sie typischerweise gewachsen wird, was höhere Temperaturen in der MBE unmöglich macht. Das Optimum in Bezug auf die Kristallqualität findet man normalerweise im Bereich $750-800^{\circ} \mathrm{C}$, je nach den exakten Randbedingungen in der Anlage. So ergab eine Optimierung die höchste Kristallqualität bei $760^{\circ} \mathrm{C}$ in der „JUEL“-Anlage und $775^{\circ} \mathrm{C}$ in der „GEN2“-Anlage.

In der MOCVD wird bei wesentlich höheren Drücken und meist höheren Wachstumsraten gearbeitet, wodurch Temperaturen im Bereich $1100-1150^{\circ} \mathrm{C}$ für das GaN-Wachstums verwendet werden können. Im Durchschnitt werden daher etwas höhere Schichtqualitäten erzielt.

Metall-Stickstoff-Verhältnis Bei konstantem Stickstoff-Angebot kann der Metall-Fluss verändert werden, um unterschiedliche Wachstumsmodi zu erzielen. Bei niedrigem MetallAngebot ist das Wachstum Metall-limitiert, d. h. die Wachstumsrate nimmt direkt proportional zum Metall-Fluss zu oder ab. Bei hohem Metall-Angebot ist das Wachstum dagegen Stickstoff-limitiert, d. h. die Wachstumsrate bleibt konstant, selbst wenn der Metall-Fluss geändert wird. Der Schnittpunkt zwischen beiden Bereichen wird als Stöchiometrie bezeichnet. Beim stöchiometrischen Wachstum ist also kein Überschuss eines der beiden Elemente vorhanden.

Es wird davon ausgegangen, dass stets Ga-polare Schichten gewachsen werden (siehe Kapitel 2.1.1). Bei stickstoffreichem Wachstum (also niedrigem Metall-Angebot) wird ein auftreffendes Metall-Atom sehr schnell durch den im Überfluss vorhandenen Stickstoff fest gebunden werden. So haben beispielsweise Zywietz et al. [59] berechnet, dass die Oberflä- 
chendiffusion von Ga-Atomen unter stickstoffreichen Bedingungen stark erniedrigt wird. Dadurch tritt dreidimensionales Wachstum auf mit typischerweise hohen Oberflächenrauigkeiten bis hin zu kolumnarem Wachstum. Der überschüssige Stickstoff kann an der Oberfläche nicht akkumulieren, er desorbiert vollständig.

Bei metallreichen Bedingungen akkumuliert das überschüssige Gallium an der Oberfläche. Von Northrup et al. [60] wurde auf Grund theoretischer Berechnungen gezeigt, dass bei einer Bedeckung von zwei Monolagen Gallium eine zusätzliche Stabilisierung auftritt, wobei die zweite Lage metallischen Charakter hat. Dieser so genannte „bilayer" wurde auch experimentell verifiziert $[61,62,63]$. Dort sind Wachstumsfenster gefunden worden, bei denen sich stabile Konfigurationen mit einem oder zwei Monolagen Ga ausbilden. Erst bei sehr hohen Ga-Flüssen tritt tatsächlich eine weitere Akkumulation mit anschließender Tröpfchenbildung ein. Die höchsten Kristallqualitäten sind im Bereich des „,bilayers“ erreichbar [64, 58]. Auch theoretische Berechnungen deuten auf eine erhöhte Beweglichkeit der N-Atome unter diesen Bedingungen hin [65].

GaMnN und das „spider web“ Sobald man zusätzlich zum Ga ein weiteres Metall anbietet mit dem Ziel, eine Dotierung im Prozentbereich durchzuführen (also eher eine Legierung anstrebt), kommt ein weiterer Parameter hinzu. Im vorliegenden Fall betraf das hauptsächlich die GaMnN-Proben, deren Wachstum ausführlich studiert wurde [56, 66]. Der im letzten Absatz „Metallfluss“ genannte Parameter setzt sich aus dem Ga- und dem Mn-Fluss zusammen, und gerade bei hohen Mn-Anteilen sind die Aussagen des letzten Absatzes nicht mehr gültig. Zur Darstellung der Wachstumsbedingungen einer Probe wurden in der Arbeitsgruppe die so genannten ,spider webs“ entwickelt, an denen sich die wichtigsten Parameter sofort ablesen lassen (siehe Abbildung 3.3).

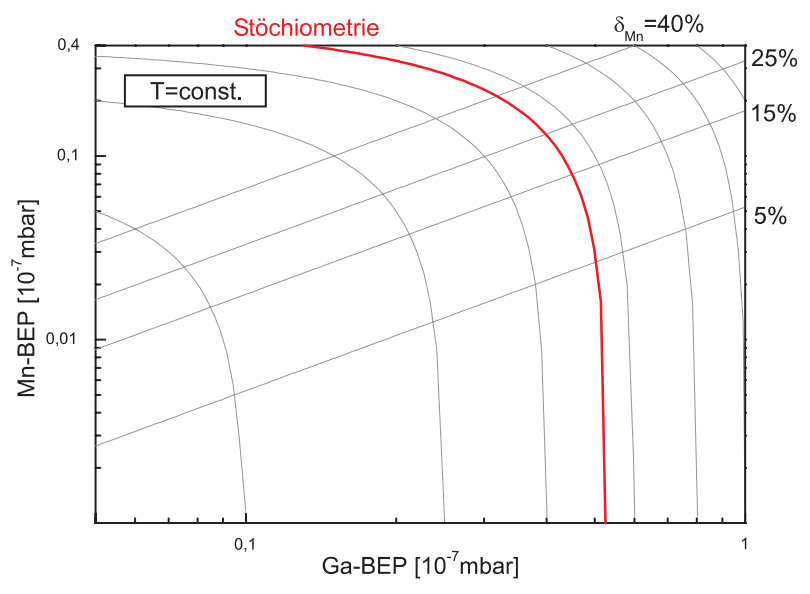

Abbildung 3.3: Darstellung des Parameter-Raums als „spider web“.

Dabei wird auf der y-Achse das Mn-Angebot dem Ga-Angebot auf der x-Achse gegenübergestellt. Punkte auf dieser x-y-Fläche entsprechen den Wachstumsbedingungen einer bestimmten Probe. Solche Diagramme wurden für jede untersuchte Wachstumstemperatur erstellt, durch diese drei Parameter werden die grundlegenden Eigenschaften der Probe festgelegt. Das Stickstoff-Angebot wurde wie erwähnt nicht variiert. Auf den Geraden in 
Abbildung 3.3 liegen die Proben, bei denen jeweils das Verhältnis $\delta_{M n}=\Phi_{M n} /\left(\Phi_{M n}+\Phi_{G a}\right)$ von Mn- zu Gesamtmetall-Fluss konstant gehalten wurde. Nur der absolute GesamtmetallFluss variiert dann. Auf den anderen Kurven liegen Proben, bei denen der GesamtmetallFluss konstant gehalten wurde und dafür $\delta_{M n}$ variiert wurde. Die rote Kurve markiert dabei genau die Kurve, bei der stöchiometrisches Wachstum für eine reine GaN-Probe erreicht wird. Links unterhalb liegen also Proben, die stickstoffreich gewachsen wurden, rechts oberhalb die metallreich gewachsenen.

\subsection{GaN auf verschiedenen Substraten}

Ein wesentlicher Punkt beim Optimieren der Wachstumsparameter von GaN ist die Wahl eines geeigneten Substrates. Dies ist ein komplexes Themengebiet, was in mehreren Übersichtsartikeln erschöpfend diskutiert wird (siehe z. B. [67, 68]). Es soll daher hier nur eine kurze Zusammenfassung gegeben werden. Häufig verwendete Substrate in der GaNEpitaxie sind in Tabelle 3.1 mit ihrem wichtigsten physikalischen Eigenschaften aufgelistet. Optimal wäre natürlich Homoepitaxie, also Wachstum auf GaN-Substraten, welche allerdings extrem teuer sind. Dasselbe gilt für $\mathrm{SiC}$, welches die beste Annäherung an die GaN-Eigenschaften bietet. Für aufwändige Testreihen mit vielen Proben kommen daher eigentlich nur die kostengünstigen Alternativen $\mathrm{Si}(111)$ und Saphir in Frage.

\begin{tabular}{|l||c|c|c|c|}
\hline physikalische Größe & GaN & 6H-SiC & Saphir & Si \\
\hline Gitterkonstante a $[\AA]$ & 3.189 & 3.0806 & 4.765 & 5.43102 \\
Gitterkonstante c $[\AA]$ & 5.186 & 15.1173 & 12.982 & \\
thermischer Ausdehnungskoeffizi- & 5.6 & 4.46 & 8.24 & 2.616 \\
ent $\|$ a $\left[10^{-6} / \mathrm{K}\right]$ & & & & \\
Schmelztemperatur $[\mathrm{K}]$ & 2791 & & 2030 & 1687 \\
Wärmeleitfähigkeit $[\mathrm{W} / \mathrm{cm} \cdot \mathrm{K}]$ & 1.3 & & 0.25 & 1.56 \\
Bandlücke $E_{g}[\mathrm{eV}]$ & 3.44 & 3.02 & $8.1-8.6$ & 1.124 \\
\hline
\end{tabular}

Tabelle 3.1: Wichtige Eigenschaften von GaN (siehe Kapitel 2.1) im Vergleich zu verschiedenen Substraten nach [68]

Silizium Si-Substrate sind leicht und in hoher Qualität erhältlich. Weiterhin haben sie den interessanten technologischen Aspekt, dass bei Wachstum auf Si-Substraten eine gewisse „Kompatibilität“ zur herkömmlichen Halbleitertechnologie gegeben ist. Es sind also integrierte Schaltkreise möglich, die teilweise das Si nutzen und teilweise die darauf deponierte Schicht. Allerdings wird in der Industrie typischerweise mit (100)-orientiertem Si gearbeitet, während das Wachstum von wurtzitischem GaN nur auf der (111)-Oberfläche in akzeptabler Qualität möglich ist. Eine volle Übertragbarkeit ist daher nicht gewährleistet.

Weiterhin ist die Gitterfehlanpassung zu GaN relativ groß und auch die thermischen Ausdehnungskoeffizienten unterscheiden sich deutlich (siehe Tabelle 3.1). Daher werden 
meistens Pufferschichten verwendet, um das Wachstum zu verbessern. Auch in den vorliegenden Proben kamen bei Einsatz eines Si-Substrates AlN-Puffer zum Einsatz. Trotzdem sind GaN-Schichten auf Si-Substraten auf Grund der unterschiedlichen thermischen Ausdehnungskoeffizienten meist tensil verspannt und weisen eine vergleichsweise schlechte Materialqualität auf. Das genaue Probendesign ist in Abbildung 3.4a) dargestellt.
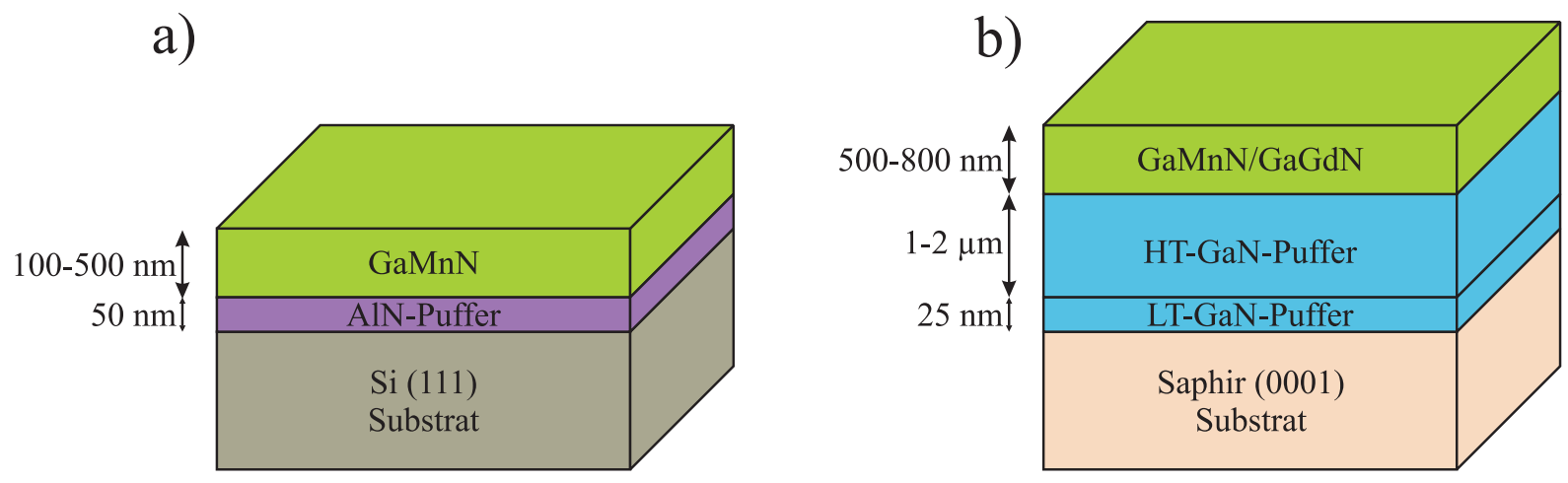

Abbildung 3.4: Aufbau a) der GaMnN-Proben auf Si-Substrat und b) der GaMnN- und GaGdN-Proben auf Saphir-Substrat.

Saphir mit MOCVD-Puffer Saphir, also einkristallines Aluminiumoxid, ist nach wie vor das am weitesten verbreitete Substrat in der GaN-Epitaxie. Es ist kommerziell erhältlich und wurde schon in den 1960er Jahren in der GaN-Epitaxie verwendet. Auf Grund der hohen Gitterfehlanpassung wächst das GaN mit einer um $30^{\circ}$ verdrehten Einheitszelle auf dem Saphir-Substrat, so dass die Fehlanpassung von 33\% auf $13.9 \%$ reduziert wird. Durch den großen thermischen Ausdehnungskoeffizienten von Saphir sind GaN-Schichten auf diesem Substrat meist kompressiv verspannt. Dies kann durch geeignete Pufferschichten teilweise ausgeglichen werden, ebenso wie dadurch die Schichtqualität drastisch erhöht werden kann. Als Puffer werden hier häufig GaN- oder AlN-Schichten benutzt, die bei niedrigen Temperaturen gewachsen werden.

Einige der GaMnN- und alle GaGdN-Schichten, die im Rahmen dieser Arbeit untersucht wurden, sind auf Saphir-Substrat gewachsen. Diese Schichten wurden auf eine GaN-Schicht deponiert, die vorher mittels MOCVD unter optimierten Wachstumsbedingungen hergestellt worden war. Die MOCVD-GaN-Schicht bestand dabei aus einem dünnen GaN-Puffer, der bei niedrigen Temperaturen $\left(550^{\circ} \mathrm{C}\right)$ gewachsen wird und einer dicken GaN-Schicht, die bei $1125^{\circ} \mathrm{C}$ gewachsen wird. Das MOCVD-Wachstum wurde im Rahmen einer Zusammenarbeit vom Forschungszentrum Jülich durchgeführt. Diese MOCVD-gewachsenen Schichten werden im Folgenden nur noch als GaN-Puffer bezeichnet. Das genaue Probendesign ist in Abbildung 3.4b) dargestellt. Durch die hochqualitativen GaN-Pufferschichten ist auf diesen Substraten trotz der nach wie vor vorhandenen kompressiven Verspannung eine sehr hohe Schichtqualität erzielbar, wie sich an Hand der in dieser Arbeit präsentierten Ergebnisse zeigt. 


\section{Experimentelle Methoden}

Im Rahmen dieser Arbeit kamen verschiedene experimentelle Methoden zur Charakterisierung der gewachsenen Schichten zum Einsatz. Der Schwerpunkt lag dabei auf der optischen Charakterisierung, aber auch einige strukturelle Untersuchungen wurden durchgeführt. Im Folgenden sollen diese Methoden vorgestellt werden.

\subsection{Photolumineszenz (PL)}

Photolumineszenz ist das Verhalten eines Materials, unter Lichteinstrahlung selbst Licht einer anderen Wellenlänge zu emittieren. Die Anregung erfolgt typischerweise durch einen Laser, während das emittierte Licht gesammelt und spektral analysiert wird.

\subsubsection{Lumineszenz in Halbleitern}

Ein Halbleiter wird dadurch charakterisiert, dass eine Energielücke $E_{g}$ zwischen dem vollständig besetzten Valenzband und dem unbesetzten Leitungsband existiert. Durch geeignete Anregung wird ein Elektron aus dem Valenzband in das Leitungsband angehoben. Dabei entsteht ein Loch im Valenzband. Die so erzeugten Elektronen und Löcher werden schließlich wieder rekombinieren, was allerdings auf verschiedene Arten und Weisen erfolgen kann, den so genannten Rekombinationskanälen. Die wichtigsten werden schematisch in Abbildung 4.1 dargestellt.

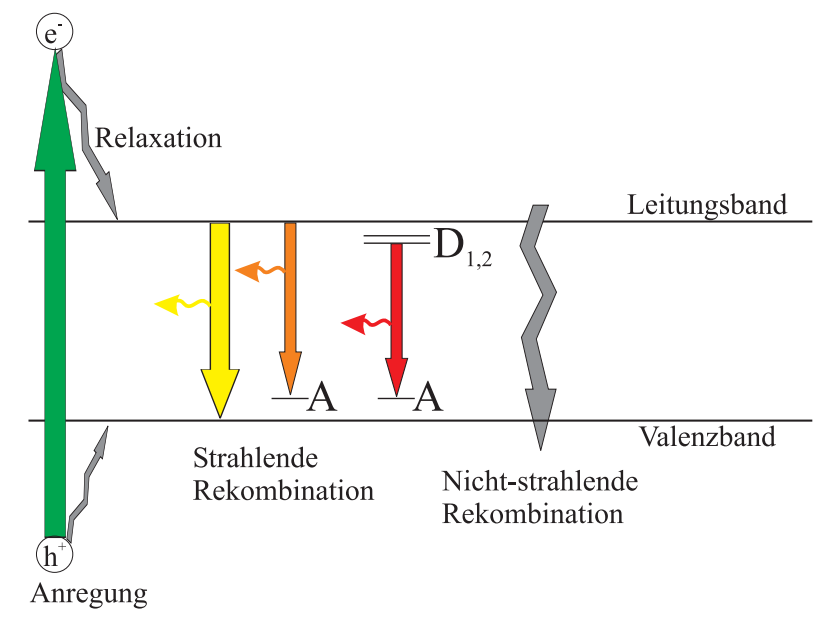

Abbildung 4.1: Verschiedene Rekombinationskanäle für angeregte Elektronen und Löcher in Halbleitern. 
Die Anregung kann auf verschiedene Arten erfolgen, z. B. durch Bestrahlen mit hochenergetischen Elektronen (Kathodolumineszenz), elektrischer Injektion von Elektronen und Löchern (Elektrolumineszenz) oder Bestrahlen mit Licht der Energie $E=\hbar \omega>E_{g}$ (Photolumineszenz), wie es in der vorliegenden Arbeit durchgeführt wurde. Als Lichtquelle dient normalerweise ein Laser, da dieser zum Einen sehr gut fokussierbar und zum Anderen monochromatisch ist. Unerwünschte Reflexionen des Laserlichtes in die Detektionsoptik können im gemessenen Spektrum daher sehr einfach identifiziert werden. Der $k$-Vektor der absorbierten Photonen ist vernachlässigbar klein gegenüber dem der Elektronen und Löcher. Das bedeutet, dass optische Übergänge nahezu senkrechten Übergängen in der Bandstruktur entsprechen, wie in Abbildung $4.2 \mathrm{zu}$ sehen ist.

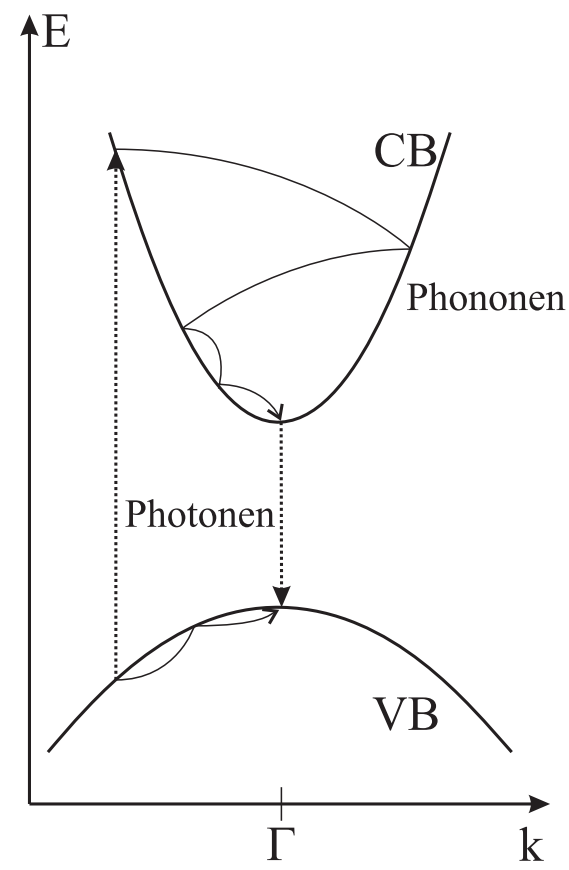

Abbildung 4.2: Absorption eines Photons und Relaxation der entstehenden Elektronen und Löcher durch Phononen.

Direkt im Anschluss an die Absorption werden die Elektronen und Löcher thermalisieren, d. h. sie relaxieren unter Aussenden optischer und akustischer Phononen zu den jeweiligen Bandextrema. Dieser Prozess ist spielt sich typischerweise auf der PicosekundenZeitskala ab, und damit mehrere Größenordnungen schneller als optische Übergänge. Im idealen Kristall können Elektronen und Löcher nun nach einer gewissen Lebensdauer strahlend, also unter Aussenden eines Photons, rekombinieren. Befinden sich Defekte im Kristall, z. B. Donatoren und Akzeptoren wie in Abbildung 4.1 dargestellt, so können diese die Elektronen und Löcher einfangen. Rekombinieren diese eingefangenen Ladungsträger mit einem freien Gegenstück, so erniedrigt sich die Energie des abgestrahlten Photons um deren Bindungsenergie. Beim Donator-Akzeptor-Paar-Übergang (DAP) erhöht ein zusätzlicher Coulomb-Term die Photonenenergie, da die beiden (vorher neutralen) Störstellen nach der Rekombination unterschiedliche Ladung tragen: 


$$
\begin{array}{r}
D^{0}+A^{0} \rightarrow D^{+}+A^{-}+\hbar \omega \\
\hbar \omega=E_{g}-E_{A}-E_{D}+\frac{e^{2}}{4 \pi \epsilon \epsilon_{0} R}
\end{array}
$$

Dabei sind $E_{A}$ und $E_{D}$ die Bindungsenergien der Ladungsträger an Donator und Akzeptor und $\epsilon$ die statische Dielektrizitätskonstante des Halbleiters. $R$ ist der Abstand der beiden Störstellen, der auf Grund der kristallinen Struktur nur bestimmte, diskrete Werte annehmen kann.

Eine andere Möglichkeit Lumineszenz zu erhalten sind intra-atomare Übergänge von Fremdatomen. Wenn diese Fremdatome in nicht zu hoher Konzentration vorliegen, sind die Energieniveaus von Orbitalen, die an keiner Bindung zur umgebenden Matrix beteiligt sind, sehr scharf. Dies tritt typischerweise bei seltenen Erden auf, deren $4 f$-Niveaus teilweise besetzt sind, aber durch deren stark lokalen Charakter fast nicht mit der Umgebung wechselwirken. Wie im freien Atom können nun so genannte intra- $4 f$-Übergänge stattfinden. Sie sind zwar auf Grund der normalen Auswahlregeln für Dipolübergänge verboten, diese werden allerdings durch die schwache Beimischung anderer Wellenfunktionen (geringer Anteil von $s^{-}, p$ - und $d$-Charakter) aufgehoben. Das Kristallfeld der Matrix (siehe Kapitel 2.2) wirkt lediglich wie eine schwache Störung.

Auch Übergangsmetalle können intra-atomare Übergänge zeigen. Hier sind die teilweise besetzten $d$-Orbitale für die Lumineszenz verantwortlich, die allerdings weit weniger von der Umgebung abgeschirmt sind als die $f$-Orbitale der seltenen Erden. Als Konsequenz ändern die $d$-Orbitale ihren Symmetrie-Charakter im Kristallfeld, wie in Kapitel 2.2 beschrieben.

Die Anregung von intra-atomaren Übergängen kann auf verschiedene Art und Weise geschehen. Zum Einen ist eine direkte Anregung möglich, das Photon wird also vom Fremdatom direkt absorbiert, wodurch das Atom in einen angeregten Zustand angehoben wird. Dieser angeregte Zustand wird nun strahlend oder nicht-strahlend in niedrigere angeregte Zustände relaxieren bis er wieder im Grundzustand ist. Dieser Prozess ist aber nur möglich, wenn die Photonenenergie genau der Energiedifferenz zwischen Grund- und angeregtem Zustand entspricht. Wenn außerdem die Matrix diese Energie absorbiert wird er sehr ineffizient. Eine zweite Möglichkeit ist der so genannte Dexter-Energietransfer [69], bei dem die Anregung über verschiedene Defekte (z. B. tiefe Störstellen) im Halbleiter übertragen werden kann, bis es eine strahlende Rekombination gibt. Weiterhin gibt es „Auger-artige“ Prozesse [70]. Dabei rekombiniert ein Exziton (siehe Kapitel 4.1.2) und gibt seine Energie teilweise an das Fremdatom ab, das dadurch in einen angeregten Zustand angehoben wird. Die Restenergie wird als Photon oder Phonon frei.

Im Allgemeinen spielen diese intra-atomaren Lumineszenzen nur dann eine Rolle, wenn die beteiligten Zustände innerhalb der Bandlücke des umgebenden Halbleiters liegen. Läge beispielsweise ein angeregter Zustand resonant im Leitungsband, so könnte er sehr effizient seine Energie unter Emission eines Leitungsband-Elektrons abgeben, also ohne optischen Übergang. Dies liegt auch an den typischerweise relativ langen Lebensdauern im Mikrobis Millisekundenbereich der intra-atomaren Übergänge. 
Eine grundsätzlich andere Art der Rekombination von Elektron und Loch ist ausschließlich nicht-radiativ. Sind im Halbleiter viele Defekte vorhanden, die große Bereiche der Bandlücke abdecken, so können Elektronen über phononische Prozesse nicht nur bis zu den Bandextrema relaxieren, sondern über mehrere Zwischenschritte auch miteinander rekombinieren. Demnach sind hierbei keinerlei Lumineszenzen zu detektieren.

\subsubsection{Exzitonen}

Elektron und Loch spüren in einem Halbleiter eine attraktive Coulomb-Kraft, die allerdings durch das Medium abgeschirmt wird. Nimmt man nun an, dass sie sich frei bewegen können, so liegt praktisch ein Wasserstoffproblem vor. Somit existiert ein gebundener Elektron-Loch Zustand, den man Exziton nennt. Man muss allerdings unterscheiden zwischen so genannten Frenkel-Exzitonen, deren Wellenfunktion eine Ausdehnung in der Größenordnung der Gitterkonstante besitzt und Wannier-Exzitonen, die wesentlich größer sind. Bei Ersteren ist eine theoretische Behandlung schwierig, da der Einfluss des Mediums durch die Atomrümpfe und die sie umgebenden Elektronen voll berücksichtigt werden muss, während er bei letzteren einfach durch die statische Dielektrizitätskonstante $\epsilon$ beschrieben wird. Dann entspricht der Hamilton-Operator bis auf diese Konstante tatsächlich exakt dem Wasserstoffproblem. Es müssen nur noch die effektiven Massen von Elektron und Loch zur Berechnung der reduzierten Masse $\mu$ bekannt sein. Die Bindungsenergie $E_{X}$ des Exzitons ist dann:

$$
E_{X, n}=-\frac{1}{n^{2}} \frac{\mu}{m_{0} \epsilon^{2}} 13.6 \mathrm{eV}
$$

$n$ entspricht dabei der Hauptquantenzahl des Wasserstoffatoms, da auch Exzitonen angeregte Zustände besitzen. Sofern nicht anders vermerkt, wird im Folgenden immer vom Grundzustand $n=1$ ausgegangen. Setzt man die in Kapitel 2.1 (Tabelle 2.1) beschriebenen Werte für GaN ein, so ergibt sich eine Exzitonbindungsenergie von etwa $28.6 \mathrm{meV}$, was gut mit den experimentell bestimmten Werten von etwa $26.7 \pm 0.5 \mathrm{meV}$ [71] übereinstimmt. Diese gute Übereinstimmung zeigt, dass die Exzitonen in GaN tatsächlich Wannier-Exzitonen sind.

Auch andere Störstellen wie etwa neutrale Donatoren $\left(\mathrm{D}^{0}\right)$ lassen sich näherungsweise wie ein Wasserstoffatom beschreiben, wobei hier das Elektron schwach gebunden um den ortsfesten, positiv geladenen Atomrumpf delokalisiert ist. Kommt ein frei bewegliches Exziton in die Nähe eines solchen Defektes, so spürt es eine anziehende van-der-WaalsWechselwirkung und es kann sich ein so genanntes „gebundenes Exziton“ bilden. Die Energie, die bei der Rekombination eines gebundenen Exzitons frei wird, ist also

$$
\hbar \omega=E_{g}-\left|E_{X}\right|-\left|E_{B X}\right|
$$

wobei $E_{B X}$ die Bindungsenergie des Komplexes Störstelle-Exziton ist, vergleichbar einem Wasserstoffmolekül. Dieses einfache Bild reicht allerdings nicht zur näherungsweisen Berechnung von $E_{B X}$. Empirisch hat sich die so genannte „Haynes' Rule“ für Akzeptoren bzw. Donatoren ergeben [72]: 


$$
\begin{aligned}
& E_{A B X}=\alpha_{A} \cdot E_{A} \\
& E_{D B X}=\alpha_{D} \cdot E_{D}
\end{aligned}
$$

Hier stehen $E_{A B X}$ und $E_{D B X}$ für die Bindungsenergie am Akzeptor bzw. Donator, die Konstante $\alpha$ ist materialspezifisch. Sie ergab sowohl für Akzeptoren als auch für Donatoren in Silizium etwa den Wert 0.1 [72]. Für GaN ergeben sich $\alpha_{A}=0.057$ und $\alpha_{D}=0.21$ [73].

Des weiteren können Exzitonen auch gebundene Zustände mit tiefen Störstellen eingehen, wie sie beispielsweise durch strukturelle Defekte verursacht werden. Die Bindungsenergien sind dann auch deutlich höher als bei flachen Störstellen, folgen aber nicht mehr Haynes' Rule.

\subsubsection{Lumineszenz von GaN}

Mit seiner Bandlücke von $3.5 \mathrm{eV}$ bei tiefen Temperaturen liegen die typischen Lumineszenzen von GaN vom nahen UV-Bereich über den sichtbaren Bereich bis in den nahen IR-Bereich. Sie lassen sich unterteilen in intrinsische Lumineszenz, die auch in idealem GaN auftreten würde (im Wesentlichen freie Exzitonen) und defekt-bedingte Lumineszenz, die auf Verunreinigungen zurückzuführen ist. Letztere kann weiter unterteilt werden in Lumineszenzen, die auch in nominell undotiertem Material fast immer präsent sind wie gebundene Exzitonen und die so genannte gelbe Lumineszenz, als auch solche, die erst nach Dotierung mit Fremdatomen erscheinen und oftmals charakteristisch für diese Atome sind wie etwa intra-atomare Übergänge. Häufig vorkommende oder für diese Arbeit wichtige Lumineszenzen sind in Tabelle 4.1 zusammengefasst.

\begin{tabular}{|c||c|l|}
\hline Position des Maximums $[e V]$ & Abkürzung & Kommentar \\
\hline 3.478 & FXA & Freies Exziton A \\
3.471 & DBX & Donator-gebundenes Exziton \\
3.466 & ABX & Akzeptor-gebundenes Exziton \\
$3.41-3.42$ & $Y_{2}$ & Strukturell gebundenes Exziton \\
$3.35-3.36$ & $Y_{4}$ & Strukturell gebundenes Exziton \\
$3.30-3.32$ & $Y_{6}$ & Strukturell gebundenes Exziton \\
$3.21-3.23$ & $Y_{7}$ & Strukturell gebundenes Exziton \\
$3.26-3.28$ & UVL & DAP- oder e-A-Übergang \\
$2.7-3.0$ & BL & Blaue Lumineszenz \\
$2.4-2.6$ & GL & Grüne Lumineszenz \\
$2.2-2.3$ & YL & Gelbe Lumineszenz \\
$1.7-2.0$ & RL & Rote Lumineszenz \\
1.30 & & Scharfe Fe-Lumineszenz \\
\hline
\end{tabular}

Tabelle 4.1: Wichtige Lumineszenzlinien und -bänder in GaN (siehe [73] und dortige Referenzen) 
Auf Grund seiner Wurtzitstruktur hat GaN ein Leitungsband und drei nicht-entartete Valenzbänder am $\Gamma$-Punkt (siehe Kapitel 2.1.3). Daher können sich drei verschiedene Arten von Exzitonen bilden, die von niedrigster zu höchster Rekombinationsenergie mit $\mathrm{A}, \mathrm{B}$ und $\mathrm{C}$ bezeichnet werden. Die Lage der Bänder ist allerdings von der Deformation des Kristalls abhängig, die durch Verspannungen beim Wachstum oder dem nachfolgenden Abkühlen verursacht werden. So wird die Bandlücke bei einer kompressiven biaxialen Deformation in der c-Ebene größer, bei tensilen Deformationen kleiner. Die Deformation in der senkrechten Richtung, also entlang der c-Achse, ist dabei entgegengesetzt. Auch die relative Position der A-, B- und C-Exzitonen wird dadurch geändert, bis hin zu einem crossing der A- und B-Exzitonen bei starken tensilen Deformationen, wie in Abbildung 4.3 dargestellt. Experimentell ist das C-Exziton mittels PL nur selten beobachtbar, da es resonant mit freien ,Streu“zuständen des A-Exzitons liegt, sprich oberhalb der eigentlichen Bandlücke.

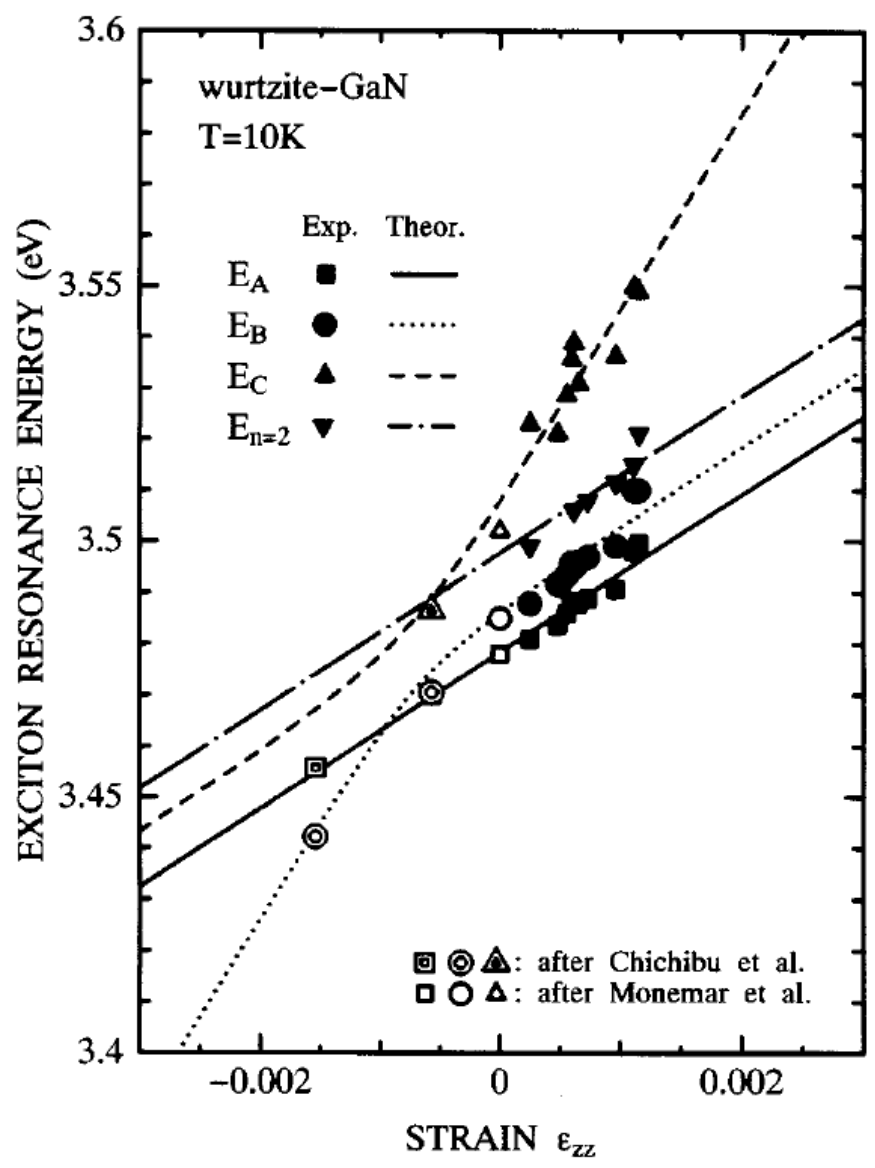

Abbildung 4.3: Abhängigkeit der Exzitonenenergien von der Deformation in c-Richtung nach [74]

Nominell undotiertes GaN ist immer n-leitend und auch in den PL-Spektren ist nahe der Bandkante normalerweise das donatorgebundene Exziton das dominierende Merkmal. 
Bei p-Dotierung kann aber auch das akzeptorgebundene Exziton dominieren oder in hochqualitativen Proben das freie Exziton. Im Bereich zwischen 3.45 und $3.2 \mathrm{eV}$ sind einige Linien bekannt, die nur manchmal auftreten und nicht eindeutig identifiziert sind. Sie werden im Folgenden mit $Y_{i}$ bezeichnet (siehe Tabelle 4.1). Meist werden sie Exzitonen zugeordnet, die an strukturelle Defekte oder ähnliche tiefe Störstellen gebunden sind.

Ein häufig zu findendes Signal befindet sich bei etwa $3.27 \mathrm{eV}$. Es ist einem DonatorAkzeptor-Paar-Übergang zuzuordnen und wird daher meist mit DAP-Band oder UVL (für ultra-violet luminescence) bezeichnet, wobei es bei höheren Temperaturen seinen Charakter ohne klaren Übergang zu einem e-A(Elektron-Akzeptor)-Übergang ändert. Seine Form ist sehr asymmetrisch mit einer ausgeprägten Verbreiterung auf der niederenergetischen Flanke. Außerdem befinden sich dort jeweils im Abstand von etwa $91 \mathrm{meV}$ Repliken dieses Signals, die durch gleichzeitige Emission eines oder mehrerer LO-Phononen entstehen. Der beteiligte Donator hat nach Reshchikov et al. [75] eine Bindungsenergie von $E_{D}=32 \mathrm{meV}$, was relativ gut zum $\mathrm{O}_{N}$ Donator $\left(E_{D}=33 \mathrm{meV}\right)$ passt. Aber auch $\mathrm{Si}_{G a}$ $\left(E_{D}=30 \mathrm{meV}\right)$ kann nicht ausgeschlossen werden. Der Akzeptor ist nicht klar identifiziert, aber da er für GaN relativ flach ist $\left(E_{A} \approx 220 \mathrm{meV}\right)$, kommen im Wesentlichen $\mathrm{Mg}_{G a}, \mathrm{Si}_{N}$ und $\mathrm{C}_{N}$ in Frage.

Eine weitere Lumineszenz, die in praktisch jeder GaN-Probe zu finden ist, wird als so genannte ,gelbe Lumineszenz" (YL) bezeichnet. Sie besteht aus einem Band bei etwa 2.20 bis $2.25 \mathrm{eV}$ mit einer Halbwertsbreite von etwa 350 bis $450 \mathrm{meV}$. Allerdings können sich diese Zahlen zwischen verschiedenen Proben durchaus signifikant unterscheiden. Der Ursprung dieser Lumineszenz wird einem Übergang zugeordnet, bei dem ein Elektron aus dem Leitungsband oder von einem flachen Donator mit einem Loch von einem tiefen Akzeptor rekombiniert. Da sich diese Lumineszenz durch eine Vielzahl von implantierten Elementen verstärken lässt [76], muss der tiefe Akzeptor mit einem intrinsischen und unspezifischen Defekt zusammenhängen. Durch theoretische Überlegungen und Positronen-Vernichtungs-Experimente wird dieser Defekt als Gallium-Leerstelle $\mathrm{V}_{G a}$ identifiziert, die sich besonders in n-leitendem GaN leicht bildet (siehe z. B. den Übersichtsartikel von Reshchikov et al. [73]). $V_{G a}$ ist für sich alleine schon ein tiefer Akzeptor, allerdings kann er sehr leicht Komplexe mit anderen Defekten bilden, wie etwa $\mathrm{O}_{N}$ oder $\mathrm{C}_{G a}$. Auch eine Anlagerung von $\mathrm{V}_{G a}$ oder eines der beschriebenen Komplexe an einen ausgedehnten strukturellen Defekt ist ein möglicher Kandidat für den tiefen Akzeptor der gelben Lumineszenz.

Die große Breite der YL könnte im Prinzip durch viele verschiedene Defekte mit einer breiten Verteilung erklärt werden. Experimentelle Befunde deuten aber eher auf einen einzigen Defekt-Typ hin, der durch eine starke Elektron-Phonon-Kopplung verbreitert wird. Zur Beschreibung benutzt man hierbei ein Konfigurations-Koordinaten Modell, wie es in Kapitel 2.2.3 beschrieben wurde (siehe Abbildung 4.4).

Die Minima von Grund- und angeregtem Zustand der YL liegen etwa $2.6 \mathrm{eV}$ auseinander, allerdings ist die direkte Anregung bei etwa $3.2 \mathrm{eV}$ am effektivsten, was durch PLE-Experimente belegt ist [77, 78]. Der angeregte Zustand relaxiert dann phononisch in sein Minimum, von wo ein Übergang in den Grundzustand im Mittel $2.2 \mathrm{eV}$ freisetzt, dem Maximum der gelben Lumineszenz. Die Breite erklärt sich also durch die sehr unterschiedliche Zahl an Phononen, die bei der Photonenemission gleichzeitig emittiert werden. 


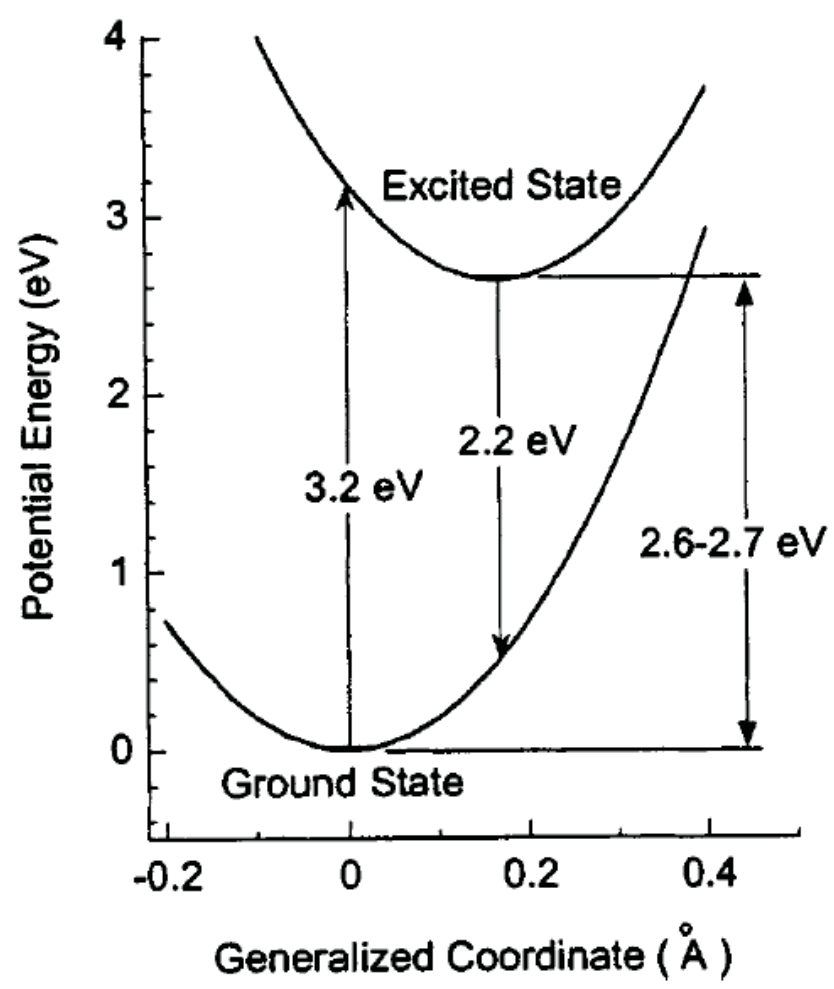

Abbildung 4.4: Eindimensionales Konfigurations-Koordinaten Diagramm für die YL in GaN nach [73]

Bei bestimmten Wachstumsbedingungen wird anstelle der gelben Lumineszenz manchmal auch eine rote oder grüne Lumineszenz beobachtet, die meist ähnlichen Ursachen zugeschrieben wird, nur mit einem etwas anderen Akzeptor-Komplex [73]. Auch die Art des Wachstums, ob die Probe also durch MBE, MOCVD, HVPE oder andere Verfahren hergestellt wurde, spielt hier eine große Rolle.

\subsubsection{Experimenteller Aufbau}

Die zu erwartende Lumineszenz von GaMnN liegt im Bereich vom nahen UV der Bandkante bis zum nahen IR von intra-3d-Übergängen. Daher wurde ein PhotolumineszenzSystem aufgebaut, mit dem dieser Bereich optimal abgedeckt werden kann. Es besteht im Wesentlichen aus dem Laser zur Anregung, dem Kryostat zur Probenkühlung und dem Spektrometer mit Detektor zur Analyse der Lumineszenz und ist schematisch in Abbildung 4.5 gezeigt.

Der Laser ist ein HeCd-Laser der Firma Kimmon mit einer Leistung von $45 \mathrm{~mW}$ bei einer Wellenlänge von $325 \mathrm{~nm}$. Dies entspricht einer Energie von etwa $3.81 \mathrm{eV}$, was deutlich oberhalb der Bandlücke von GaN liegt, die bei tiefen Temperaturen etwa $3.50 \mathrm{eV}$ beträgt. Der Absorptionskoeffizient von GaN bei der Laserwellenlänge liegt bei $\alpha \approx 1.2$. $10^{5} \mathrm{~cm}^{-1}$, was einer Eindringtiefe von etwa $83 \mathrm{~nm}$ entspricht. Typischerweise werden also nur wenige hundert Nanometer der Probe zur Lumineszenz angeregt. Dies ist gleichzeitig 


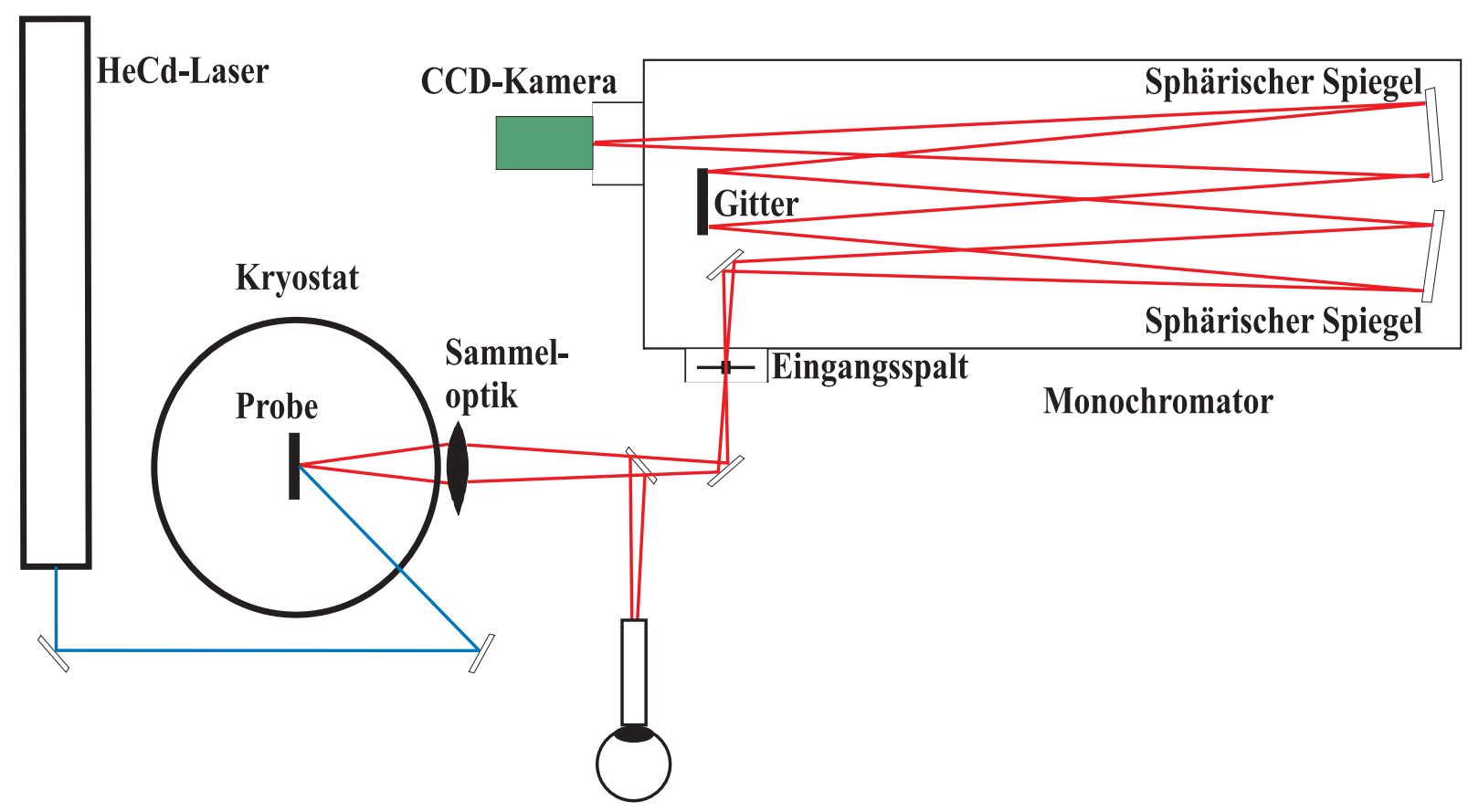

Abbildung 4.5: Schematischer Aufbau des PL-Systems.

die Größenordnung der Schichtdicke bei den hier untersuchten Proben.

Über dielektrische Spiegel und eine Linse wird das Laserlicht auf die Probe fokussiert. Die Spiegel reflektieren nur nahe der Laserlinie bei $325 \mathrm{~nm}$, so dass störende Plasmalinien des Lasers effektiv unterdrückt werden. Die Probe ist in einem Immersions-Kryostaten montiert, der mit flüssigem Helium gefüllt wird. Dieses wird durch abpumpen von $4.2 \mathrm{~K}$ noch einmal abgekühlt auf unter $2 K$, wobei es einen Phasenübergang zum suprafluiden Helium II macht. Daraus resultiert unter anderem eine unendliche Wärmeleitfähigkeit, was zur Folge hat, dass sich in der flüssigen Phase beim Sieden keine Blasen mehr bilden, sondern die He-Atome direkt von der Oberfläche abdampfen. Dies ist ein wichtiger Effekt, der für eine hohe Abbildungsqualität der Optik sorgt.

Mittels eines Objektives wird die Probe nun auf den Eingangsspalt des Gitterspektrometers abgebildet. Vor dem Eingangsspalt können noch verschiedene Farbfilter montiert werden, um im roten und infraroten Spektralbereich die zweite Ordnung der Laserlinie sowie der ultravioletten und blauen Lumineszenzen herauszufiltern. Das Gitterspektrometer von „Acton Research“ ist mit drei verschiedenen Gittern ausgestattet, die über eine drehbare Aufhängung gewechselt werden können. Zur Auswahl stehen Gitter mit 300, 1200 und 1800 Linien pro Millimeter, wobei das 1200er Gitter mit einem Blaze-Winkel von $750 \mathrm{~nm}$ nur für den nahen Infrarotbereich optimiert ist, während die anderen beiden mit einem Blaze-Winkel von $500 \mathrm{~nm}$ für den gesamten sichtbaren Bereich und die angrenzenden UV- und IR-Bereiche geeignet ist. Sofern nicht anders vermerkt, kam bei den Messungen immer das 1800er Gitter zum Einsatz. Als Detektor diente eine CCD-Kamera von Princeton Instruments mit $1752 \times 576$ Pixeln. Zur Verringerung der Artefakte, die beim Zusammenfügen mehrerer CCD-Bilder zu einem Spektrum auftreten, wurde der 
horizontale Bereich bei Übersichtsmessungen immer auf 1352 Pixel eingeschränkt.

Alle optischen Komponenten sind für den sichtbaren Spektralbereich optimiert. Sie gewährleisten hohe Transmission bzw. Reflexion von $350-1000 \mathrm{~nm}$, also dem Bereich, in dem Messungen durchgeführt wurden. Auch der CCD-Detektor ist auf diesen Wellenlängenbereich ausgelegt. 


\subsection{Raman-Streuung}

Wenn eine Lichtwelle durch ein Medium propagiert, so kann ein Teil von ihr elastisch oder inelastisch gestreut werden. Elastische Streuung kann zum Beispiel durch Defekte ausgelöst werden, allerdings wird dabei die Energie des Lichtes nicht geändert und dieser Fall soll hier nicht weiter betrachtet werden. Allerdings kann die Lichtwelle auch an einem Phonon gestreut werden, wobei sie Energie gewinnt oder verliert. Diesen Prozess nennt man Raman-Streuung und er soll im Folgenden genauer beschrieben werden.

Theoretische Beschreibung Die einfallende Lichtwelle kann durch ein sinusförmiges elektrisches Feld $\vec{E}$ beschrieben werden:

$$
\vec{E}(\vec{r}, t)=\vec{E}_{i}\left(\vec{k}_{i}, \omega_{i}\right) \cos \left(\vec{k}_{i} \cdot \vec{r}-\omega_{i} t\right)
$$

Dabei ist $\vec{E}_{i}$ die Amplitude der einfallenden Welle, $\vec{k}_{i}$ und $\omega_{i}$ sind deren Wellenvektor bzw. Frequenz. In einem dielektrischen Medium mit Suszeptibilität $\chi$ wird durch diese Welle eine Polarisation $\vec{P}$ induziert mit:

$$
\begin{aligned}
\vec{P}(\vec{r}, t)= & \vec{P}\left(\vec{k}_{i}, \omega_{i}\right) \cos \left(\vec{k}_{i} \cdot \vec{r}-\omega_{i} t\right) \\
& \vec{P}\left(\vec{k}_{i}, \omega_{i}\right)=\chi\left(\vec{k}_{i}, \omega_{i}\right) \vec{E}_{i}\left(\vec{k}_{i}, \omega_{i}\right)
\end{aligned}
$$

Eine Phonon in diesem Medium lenkt nun die Atome gemäß

$$
\vec{Q}(\vec{r}, t)=\vec{Q}\left(\vec{q}, \omega_{0}\right) \cos \left(\vec{q} \cdot \vec{r}-\omega_{0} t\right)
$$

aus, wobei $\vec{q}$ und $\omega_{0}$ sein Wellenvektor bzw. seine Frequenz sind. Diese Gitterschwingungen ändern nun ihrerseits die Suszeptibilität $\chi$. Da die Auslenkungen klein gegenüber der Gitterkonstante sind, kann diese Änderung durch eine Taylor-Entwicklung in $\vec{Q}$ beschrieben werden:

$$
\chi\left(\vec{k}_{i}, \omega_{i}, \vec{Q}\right)=\chi_{0}\left(\vec{k}_{i}, \omega_{i}\right)+\left(\frac{\partial \chi}{\partial \vec{Q}}\right)_{0} \vec{Q}(\vec{r}, t)+\ldots
$$

$\chi_{0}$ ist dabei die elektrische Suszeptibilität im schwingungsfreien Kristall, während der nächste Term eine oszillierende Modulation durch das Phonon beschreibt. Höhere Ordnungen werden vernachlässigt. Setzt man nun Gleichung 4.11 in 4.9 ein, so erhält man für die Polarisation einen Ausdruck von der Form:

$$
\vec{P}(\vec{r}, t, \vec{Q})=\vec{P}_{0}(\vec{r}, t)+\vec{P}_{i n d}(\vec{r}, t, \vec{Q})
$$

$\vec{P}_{0}$ beschreibt dabei den ungestörten Teil der Polarisation nach

$$
\vec{P}_{0}(\vec{r}, t)=\chi_{0}\left(\vec{k}_{i}, \omega_{i}\right) \vec{E}_{i}\left(\vec{k}_{i}, \omega_{i}\right) \cos \left(\vec{k}_{i} \cdot \vec{r}-\omega_{i} t\right)
$$

und

$$
\vec{P}_{i n d}(\vec{r}, t, \vec{Q})=\left(\frac{\partial \chi}{\partial \vec{Q}}\right)_{0} \vec{Q}(\vec{r}, t) \vec{E}_{i}\left(\vec{k}_{i}, \omega_{i}\right) \cos \left(\vec{k}_{i} \cdot \vec{r}-\omega_{i} t\right)
$$


den Anteil, der durch das Phonon induziert wird. Setzt man dort nun $\vec{Q}$ aus Gleichung 4.10 ein, so erhält man ein Produkt aus zwei Kosinus-Funktionen, das mit Hilfe der Additionstheoreme zerlegt werden kann. Man erhält:

$$
\begin{aligned}
\vec{P}_{\text {ind }}(\vec{r}, t, \vec{Q})= & \frac{1}{2}\left(\frac{\partial \chi}{\partial \vec{Q}}\right)_{0} \vec{Q}\left(\vec{q}, \omega_{0}\right) \vec{E}_{i}\left(\vec{k}_{i}, \omega_{i}\right) \\
& \times\left[\cos \left(\left(\vec{k}_{i}+\vec{q}\right) \cdot \vec{r}-\left(\omega_{i}+\omega_{0}\right) t\right)+\cos \left(\left(\vec{k}_{i}-\vec{q}\right) \cdot \vec{r}-\left(\omega_{i}-\omega_{0}\right) t\right)\right]
\end{aligned}
$$

Es werden demnach zwei unterschiedliche Wellen induziert, deren Frequenzen um $\pm \omega_{0}$, also die Phononenfrequenz, verschoben sind. Die Welle mit Frequenz $\omega_{S}=\omega_{i}-\omega_{0}$ wird als Stokes-Welle bezeichnet und diejenige mit $\omega_{S}=\omega_{i}+\omega_{0}$ als Anti-Stokes-Welle. Sie ergeben inelastisch gestreutes Licht, das in diesem Fall als Raman-gestreutes Licht bezeichnet wird, nach Chandrasekhara Venkata Raman, dem Entdecker dieses Effektes [79].

Aus der einfallenden Lichtwelle werden also Teile inelastisch gestreut, was im Spektrum als Seitenbanden sichtbar wird. Zweckmäßigerweise verwendet man daher einen Laser als Lichtquelle, da er monochromatisch ist. In einem Raman-Spektrum wird die Intensität des gestreuten Lichtes gegen die Raman-Verschiebung aufgetragen, die definiert ist als Differenz der Energien von Laser- und gestreutem Licht.

Die Stokes-verschobene Linie entspricht dabei einem Prozess, bei dem das einfallende Photon Energie an den Kristall in Form eines Phonons abgibt, während beim AntiStokes-Prozess ein vorhandenes Phonon vernichtet wird und so die Energie des Photons erhöht wird. Dieses Bild wird auch für eine quantenmechanische Beschreibung der RamanStreuung verwendet, die hier nicht vertieft werden soll. Weiterführende Details findet man beispielsweise in [80]. Eine wichtige Folgerung aus der quantenmechanischen Betrachtung ist die Tatsache, dass dann die Intensität der Stokes-Linie proportional zu $N+1$ ist, wobei $\mathrm{N}$ die Besetzungszahl der Phononenmode ist. Die Anti-Stokes-Linie ist proportional zu $N$, so dass sie bei tiefen Temperaturen verschwindet, wo es auf Grund der Nullpunktsschwingungen nach wie vor Stokes-Streuung gibt.

Für den Raman-Streuprozess gilt sowohl Impuls- als auch Energieerhaltung. Die Energieerhaltung zeigt sich in der Verschiebung der Photonenfrequenz um exakt die Phononenfrequenz. Aus der Impulserhaltung folgt eine Einschränkung für die beteiligten Wellenvektoren. Der Wellenvektor eines Photons im sichtbaren Spektralbereich ist in der Größenordnung $10^{6} \mathrm{~cm}^{-1}$, während die erste Brillouin-Zone für Phononen etwa einen Faktor 100 größere Wellenvektoren erlaubt. Die größtmögliche Änderung des Photonenvektors wird in der Rückstreugeometrie erreicht und beträgt das doppelte des Photonenvektors. Auf Grund des Erhaltungssatzes ist dies also der maximale Vektor, den das erzeugte oder vernichtete Phonon besitzen kann. Daraus folgt direkt, dass nur Phononen sehr nahe des $\Gamma$-Punktes zum Raman-Effekt beitragen können. Akustische Phononen haben in diesem Fall eine extrem geringe Energie, sie werden daher normalerweise unter dem Schlagwort Brillouin-Streuung gesondert betrachtet. Optische Phononen allerdings haben bei $k \approx 0$ Energien im Bereich $10-100 \mathrm{meV}$ für typische Halbleitermaterialien. Dies ist damit der Bereich, in dem typische Ramanspektren liegen. Meist werden in der RamanSpektroskopie die Energien in $\mathrm{cm}^{-1}$ gemessen, also in Wellenzahlen. Sie hängen mit der Energie in $\mathrm{meV}$ über $E\left[\mathrm{~cm}^{-1}\right]=e / h \mathrm{c} \cdot 10^{-5} \cdot E[\mathrm{meV}] \approx 8.0655 \cdot E[\mathrm{meV}]$ zusammen. 
Raman-Auswahlregeln Die Intensität $I_{s}$ der gestreuten Photonen ist abhängig vom $\vec{E}$ Feldvektor der gestreuten Lichtwelle $\vec{E}_{s}$ nach $I_{s} \propto\left|\overrightarrow{P_{i n d}} \cdot \overrightarrow{E_{s}}\right|^{2}$. Mit Gleichung 4.15 ergibt sich dann

$$
I_{s} \propto\left|\vec{E}_{i} \cdot R \cdot \vec{E}_{s}\right|^{2}
$$

Dabei ist $R=\left(\frac{\partial \chi}{\partial \vec{Q}}\right)_{0} \frac{\vec{Q}\left(\vec{q}, \omega_{0}\right)}{\left|\vec{Q}\left(\vec{q}, \omega_{0}\right)\right|}$ der so genannte Raman-Tensor. Er ist ein symmetrischer Tensor zweiter Ordnung. Die möglichen Phononenmoden von Wurtzit sind in Abbildung 4.6 dargestellt. Auf Grund der Symmetrie des Kristalls und der jeweiligen Phononenmode ergeben sich zusätzliche Bedingungen an den Ramantensor, die häufig zu verschwindenden Einträgen führen. Dies wurde beispielsweise für Wurtzit von Arguello et al. berechnet [81]. Damit ergeben sich die so genannten Raman-Auswahlregeln für die Phononen, die angeben, in welcher Geometrie sie mittels Ramanstreuung beobachtbar sind. Die $B_{1}^{L}$ - und $B_{1}^{H}$-Mode in Wurtzit ist auf Grund ihrer Symmetrie überhaupt nicht Raman-aktiv. Die anderen, Raman-aktiven Phononenmoden von GaN sind in Tabelle 4.2 zusammengefasst.
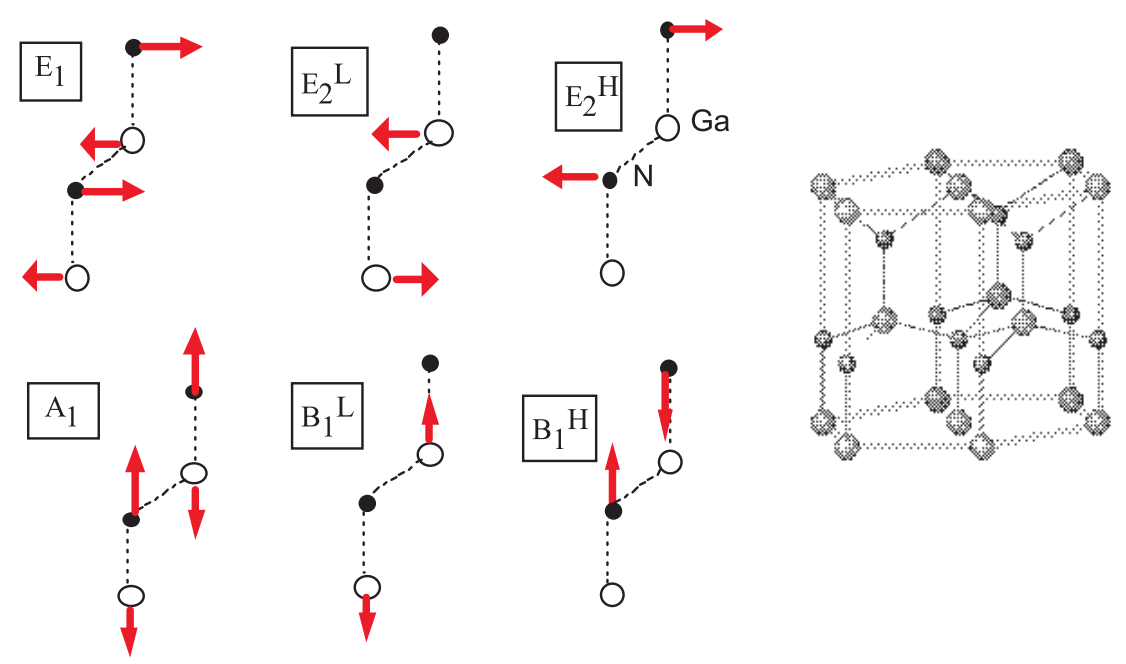

Abbildung 4.6: Phononenmoden in Wurtzit-Kristallen.

\begin{tabular}{|c|c|c|}
\hline Mode & Frequenz $\left[\mathrm{cm}^{-1}\right]$ & Mess-Geometrie \\
\hline$E_{2}^{L}$ & 144 & $x(y, y) \bar{x}, x(y, y) z, z(,) \bar{z}$ \\
$A_{1}(T O)$ & 533 & $x(z, z) \bar{x}$ \\
$E_{1}(T O)$ & 560 & $x(z, y) \bar{x}, x(y, z) y$ \\
$E_{2}^{H}$ & 569 & $x(y, y) \bar{x}, x(y, y) z, z(,) \bar{z}$ \\
$A_{1}(L O)$ & 735 & $z(y, y) \bar{z}$ \\
$E_{1}(L O)$ & 742 & $x(y, z) y$ \\
\hline
\end{tabular}

Tabelle 4.2: Phononenmoden am $\Gamma$-Punkt von Wurtzit-GaN nach [82] mit den Konfigurationen, bei denen sie sichtbar sind. Die $z$-Richtung wurde parallel zur $c$-Achse des Kristalls gewählt.

Die Spalte „Mess-Geometrie“ gibt dabei an, wie einfallendes und gestreutes Licht zueinander orientiert sein müssen, um die entsprechende Mode zu beobachten. Es wird die 
so genannte Porto-Notation verwendet, die $\overrightarrow{k_{i}}\left(\overrightarrow{e_{i}}, \overrightarrow{e_{s}}\right) \overrightarrow{k_{s}}$ geschrieben wird. $\overrightarrow{k_{i}}$ und $\overrightarrow{k_{s}}$ sind die Richtungen der einfallenden und gestreuten Photonen, $\overrightarrow{e_{i}}$ und $\overrightarrow{e_{s}}$ deren Polarisationen. Bei fehlender Polarisationsangabe ist die Mode in beiden Möglichkeiten beobachtbar.

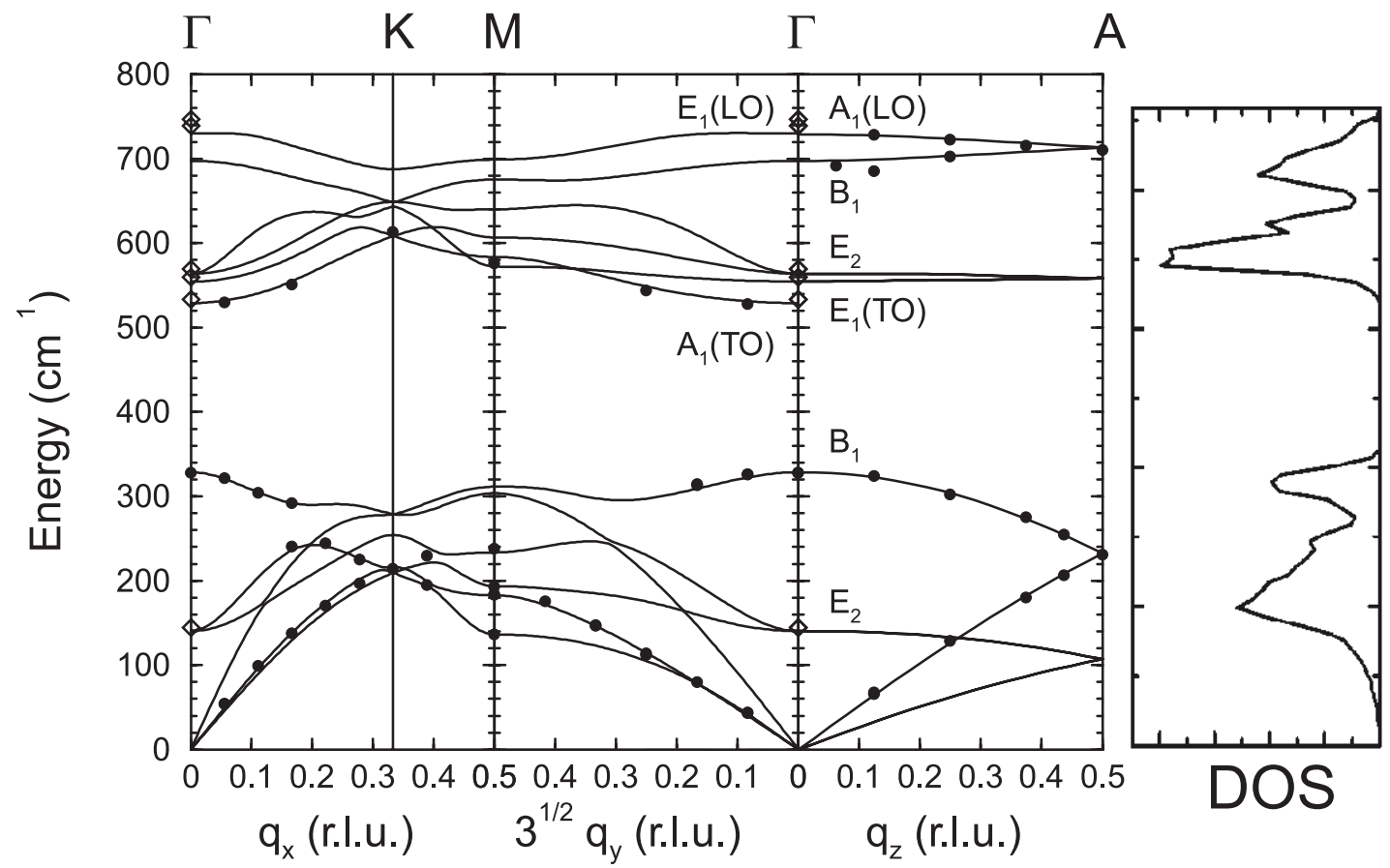

Abbildung 4.7: Phononen-Dispersionsrelation von GaN nach [83]. Durchgezogene Linien stammen aus theoretischen Berechnungen, gefüllte Symbole aus Messungen von inelastischer Röntgenstreuung. Die offenen Symbole markieren Raman-Messungen. Rechts die berechnete Phononen-Zustandsdichte nach [84]

Die gesamte Phononen-Dispersionsrelation von GaN in der Wurtzit-Struktur, wie sie in Abbildung 4.7 dargestellt ist, wurde theoretisch berechnet. Experimentell ist durch Raman-Streuung wie gezeigt nur der Bereich nahe des $\Gamma$-Punktes verifizierbar, für höhere $\vec{k}$-Vektoren sind andere Verfahren nötig, wie etwa inelastische Röntgen- oder Neutronenstreuung. Allerdings können auch Raman-Prozesse höherer Ordnung weitere Informationen liefern. Außer dem ein- und ausfallenden Photon sind dann mindestens zwei Phononen an diesen Prozessen beteiligt. Werden beide erzeugt oder vernichtet spricht man von Obertönen, wenn eines erzeugt und ein anderes vernichtet wird von Differenzmoden. Da es sich hierbei mindestens um einen Vier-Teilchen-Prozess handelt im Gegensatz zum Drei-Teilchen-Prozess des Raman-Effekts erster Ordnung ist die Intensität der sich daraus ergebenden Seitenbanden typischerweise um mehrere Größenordnungen geringer. Allerdings ist durch die Beteiligung von mehr als einem Phonon die Impulserhaltung problemlos für jeden Punkt auf der Phononen-Dispersionsrelation durch ein Phonon mit exakt entgegengesetztem $\vec{k}$-Vektor erfüllbar. Daher ähneln die erhaltenen Spektren der Phononen-Zustandsdichte.

Verspannungen und Phonon-Plasmon-Kopplung Ist ein Kristall verspannt, so kann dies Auswirkungen auf die Phononenmoden haben. So wurde beispielsweise in GaN be- 
obachtet, dass die $E_{2}^{H}$-Mode eine ausgeprägte Verschiebung proportional zur biaxialen Verspannung aufweist, was sich auch aus Abbildung 4.6 ableiten lässt. Die $A_{1}(L O)$-Mode zeigt dagegen nur eine minimale Verschiebung. Dies lässt sich allgemein durch

$$
\Delta \omega=K \sigma_{x x}
$$

ausdrücken, wobei $\Delta \omega$ die Frequenzverschiebung darstellt, $\sigma_{x x}$ die Verspannung in der Basalebene und $K$ die Proportionalitätskonstante. $K_{E_{2}^{H}}$ wurde von mehreren Gruppen experimentell bestimmt, dabei ergaben sich Werte von $2.7 \mathrm{~cm}^{-1} G P a^{-1}$ [85], $2.9 \mathrm{~cm}^{-1} \mathrm{GPa}^{-1}$ [86], 4.2 $\mathrm{cm}^{-1} \mathrm{GPa}^{-1}$ [87] und 6.2 $\mathrm{cm}^{-1} \mathrm{GPa}^{-1}$ [88]. Theoretische Berechnungen liefern einen Wert von $2.4 \mathrm{~cm}^{-1} \mathrm{GPa}^{-1}$ [89], so dass im Folgenden der niedrigste experimentelle Wert von $2.7 \mathrm{~cm}^{-1} \mathrm{GPa}^{-1}$ aus [85] verwendet wird. Ein weiterer Grund für die Wahl dieser Zahl ist, dass in dieser Publikation von Davydov et al. parallel optische Eigenschaften untersucht und PL- und XRD-Messungen mit den Raman-Daten korreliert wurden, wie es auch das Ziel der vorliegenden Arbeit ist. Genauere Details zu den Auswirkungen von Verspannungen auf die Phononenmoden finden sich beispielsweise in [90].

Kollektive Anregungen der freien Ladungsträger, die so genannten Plasmonen, können mit LO-Phononen des Gitters koppeln. Man spricht dann von gekoppelten LO-PhononPlasmon-Moden (kurz LOPPC). In GaN wurde dieser Effekt am $A_{1}(L O)$-Phonon beobachtet $[91,92]$. Während bei Ladungsträgerdichten bis etwa $10^{17} \mathrm{~cm}^{-3}$ eine relativ scharfe Linie bei $735 \mathrm{~cm}^{-1}$ beobachtet werden kann, verschiebt sich diese Linie bei höheren Konzentrationen zu höheren Energie und verbreitert sich asymmetrisch. Dieser Effekt konnte in keiner der untersuchten magnetisch dotierten GaN-Schichten beobachtet werden, die Proben hatten also freie Ladungsträgerdichten unterhalb von $10^{17} \mathrm{~cm}^{-3}$.

Lokale Moden und DARS Wird in einem Kristall ein bestimmter Anteil der Gitteratome durch Fremdatome ersetzt, so ändert das die elastischen Eigenschaften dieses Materials. In erster Näherung wird dabei angenommen, dass wie bei einem Modell mit Federn alle Federkonstanten (also Bindungsstärken) gleich bleiben und die Änderung nur durch die unterschiedliche Masse des Fremdatoms verursacht wird. Dann gibt es eine so genannte lokale Schwingungsmode $\omega_{l o c}$, die wie folgt von der abgeleiteten Mode $\omega_{0}$ des Ausgangsmaterials abhängt:

$$
\omega_{l o c}=\sqrt{\frac{\mu_{h o s t}}{\mu_{i m p}}} \omega_{0} .
$$

$\mu=\frac{m_{1} m_{2}}{m_{1}+m_{2}}$ ist dabei die reduzierte Masse eines Atompaares, wobei sich der Index „host" auf den idealen Kristall bezieht und ,imp“ auf das Fremdatom mit seinem Nachbarn. Im Fall von GaMnN, wo das Mn- ein Ga-Atom ersetzt und als nächsten Nachbarn ein N-Atom hat, ergibt sich für die $E_{2}$-Mode nach Gleichung 4.18 folgende lokale Mode:

$$
\omega_{l o c}=\sqrt{\frac{\mu_{G a-N}}{\mu_{M n-N}}} \omega_{0}=\sqrt{\frac{\frac{1}{m_{N}}+\frac{1}{m_{M n}}}{\frac{1}{m_{N}}+\frac{1}{m_{G a}}}} \omega_{0}=581.7 \mathrm{~cm}^{-1} .
$$

Für die $E_{2}^{H}$-Mode wurde dabei, wie in Tabelle 4.2 angegeben, ein Wert von $569 \mathrm{~cm}^{-1}$ angenommen. Genauere Theorien für lokale Moden finden sich beispielsweise in [93]. 
Ein weiteres Phänomen, dass in hochdotierten Kristallen beobachtet werden kann, ist die Entstehung von Unordnungs-aktivierter Raman-Streuung (DARS, nach „disorderactivated raman scattering) [94]. Dabei sind die Phononen auf Defekt-freie Bereiche beschränkt, welche typischerweise kleiner als $100 \mathrm{~nm}$ sind. Durch diese Lokalisierung werden die Auswahlregeln gelockert und insbesondere die Impulserhaltung wird abgeschwächt. Damit können auch Phononen außerhalb der Brillouin-Zonenmitte zur Raman-Streuung beitragen und es entsteht ein nahezu direktes Abbild der Phononenzustandsdichte. Auch in implantierten Proben wird dieser Effekt häufig beobachtet. 


\subsection{Weitere Messmethoden}

\subsubsection{Absorption}

Außer der Photolumineszenz kamen im Rahmen dieser Arbeit als weitere optische Messmethode die Absorption zum Einsatz. Absorption beschreibt dabei genau den komplementären Prozess zur Lumineszenz: Das System geht unter Absorption eines Photons von einem energetisch niedrig liegenden Zustand in einen höher liegenden Zustand über. Wenn die Temperatur wie im vorliegenden Fall in der Nähe der Siedetemperatur von flüssigem Helium liegt, so kommen als Ausgangszustand nur der Grundzustand und wenige, energetisch sehr nahe liegende, angeregte Zustände in Frage.

Der wesentlich Unterschied zur Photolumineszenz liegt im Fehlen eines Energietransfers, der dort nach der Anregung stattfindet, bis die Photonen-Emission aus einem metastabilen, angeregten Zustand erfolgen kann. Absorptionsprozesse finden direkt und ohne Konkurrenzprozesse statt, so dass eine quantitative Aussage hier leichter zu treffen ist. Physikalisch wird meist die Intensität $I_{t}$ des transmittierten Lichtes gemessen, aus der sich der Absorptionskoeffizient $\alpha$ wie folgt berechnet:

$$
\alpha=\frac{\ln \frac{I_{0}}{I_{t}}}{d}
$$

Dabei ist $I_{0}$ die eingestrahlte Intensität und $d$ die Dicke des absorbierenden Materials. Im Falle von Mehr-Schicht-Systemen bzw. Heterosubstraten müssen die Eigenschaften aller Schichten berücksichtigt werden und eventuelle Fabry-Perot-Oszillationen auf Grund von Brechungsindex-Unterschieden z. B. zwischen Saphir-Substrat und GaN-Schicht durch geeignete Datenanalyse entfernt werden. Eine weitere Beschränkung besteht darin, dass das Substrat bei der betrachteten Wellenlänge nicht zu stark absorbieren darf, damit noch ein messbares Signal detektiert werden kann. So waren die Proben auf Si-Substrat gänzlich ungeeignet für diese Methode, während das Saphir-Substrat mit einer nahezu konstanten, schwachen Absorption im gesamten betrachteten Spektralbereich ideale Eigenschaften aufweist.

\subsubsection{Rutherford-Rückstreuung (RBS) und Implantation}

Für mehrere Teilaspekte dieser Arbeit war der Einsatz von Ionenbeschleunigern notwendig. So mussten für die SIMS-Messungen Referenzproben hergestellt werden, die ein bekanntes Dotierprofil des entsprechenden Elements aufweisen, was am besten durch Ionenimplantation zu erreichen ist. Auch RBS (Rutherford-Rückstreuung) wird mit Hilfe eines Ionenbeschleunigers durchgeführt. Die Implantationen wurden am IONAS-Beschleuniger (IONenAnSchubser) und ADONIS-Beschleuniger (Anlage zur Deposition Niederenergetischer Ionen auf Substrate) des II. Physikalischen Instituts der Universität Göttingen durchgeführt. Die RBS-Messungen wurden im Forschungszentrum Jülich durchgeführt. Diese Beschleuniger arbeiten nach dem gleichen Grundprinzip: In der Quelle wird das gewünschte Element verdampft (wenn es nicht sowieso gasförmig ist) und ionisiert. Die entstandenen Ionen werden dann vorbeschleunigt, ehe sie durch das Magnetfeld des so 
genannten Massenseparators abgelenkt werden. Da diese Ablenkung von der spezifischen Ladung $q / m$ abhängt, kann durch einen engen Spalt nun genau ein Isotop aus dem Strahl ausgewählt werden. Danach folgt die Endbeschleunigung auf die gewünschte Energie.

Im klassischen Versuch von Rutherford wurde erstmals nachgewiesen, dass Atome aus einem winzigen, massiven Kern bestehen und einer ausgedehnten Elektronenhülle. Der beobachtete Effekt war, dass ein Strahl von $\alpha$-Teilchen zum größten Teil ungehindert durch eine hauchdünne Goldfolie drang, während ein kleiner Teil mit einer bestimmten Winkelverteilung zurückgestreut wurde. Bei RBS wird die zu untersuchende Probe einem Teilchenstrahl ausgesetzt, und zwar typischerweise nach wie vor doppelt positiv geladenem ${ }^{4} \mathrm{He}$. Abhängig von der Energie der eingestrahlten Teilchen haben diese eine Eindringtiefe im Bereich $100 \mathrm{~nm}$ bis wenige $\mu \mathrm{m}$. Diese positiv geladenen Teilchen erfahren eine abstoßende Coulomb-Wechselwirkung mit den Atomkernen, sowie inelastische Wechselwirkungen mit den Elektronenhüllen, die man sich bildlich wie Reibung vorstellen kann. Ersteres führt zu elastischen Stößen nach dem Billard-Prinzip, zweiteres zu dem so genannten Energieverlust, der die eindringenden Ionen langsam abbremst. Die Winkelverteilung und Energie der rückgestreuten Teilchen lässt sich direkt aus den Eigenschaften des Coulomb-Potentials berechnen. Sie hängt außer von den Ordnungszahlen der Stoßpartner von der kinetischen Energie des Projektils ab. Da diese wiederum von der Eindringtiefe abhängt, ist das Energiespektrum der rückgestreuten Teilchen abhängig von der elementaren Zusammensetzung der Probe und der Tiefenverteilung dieser Zusammensetzung. Mittels geeigneter Analyse-Software kann aus dem unter einem bestimmten Winkel gemessenen Energiespektrum damit die elementare Zusammensetzung dünner Schichten erhalten werden. Diese ist nicht direkt zugänglich, sondern es muss über den Vergleich der Messung mit einem Modell gearbeitet werden, bei dem nur wenige Parameter variabel sind. Im Rahmen dieser Arbeit kam das Programm RUMP [95] zur Simulation und Anpassung von theoretischen und experimentellen Spektren zum Einsatz.

Bei den Implantationen kamen einfach positiv geladene Ionen zum Einsatz. Während der Implantation wird der Strom auf dem Probenhalter gemessen und mit Hilfe eines Stromintegrators die deponierte Ladung bestimmt. Die implantierte Fluenz $F$ wird aus der Ladung $C$ und der bestrahlten Fläche von $1 \mathrm{~cm} \times 1 \mathrm{~cm}$ nach

$$
F=\frac{C}{e \cdot 1 c m^{2}}
$$

berechnet, wobei $e$ die Elementarladung ist. Zur Simulation des Implantationsprozesses kam das Programm „TRIM“ [96] zum Einsatz. Die Parameter sind dabei wie im Falle von RBS Ordnungszahl, Massenzahl und Energie des Projektils, sowie die elementare Zusammensetzung und Atomdichte der Probe. Das Programm errechnet damit den Pfad und die vollständigen Kollisionskaskaden der eindringenden Ionen mittels einer Monte-CarloSimulation ohne Berücksichtigung der exakten Kristallstruktur. Als Ergebnis erhält man ein Tiefenprofil der implantierten Spezies aus dem man mit Hilfe der bekannte Fluenz ein Konzentrationsprofil berechnen kann. Die Parameter der als SIMS-Standard implantierten GaN-Proben sind in Tabelle 4.3 angegeben.

Alle Implantationen wurden dabei unter einem Winkel von $\approx 10^{\circ}$ zur Flächennormalen der Probe durchgeführt, um so genannte Channeling-Effekte auszuschließen. Darunter 


\begin{tabular}{|c|c|c|c|c|}
\hline Element & $\begin{array}{c}\text { Atommasse } \\
{[u]}\end{array}$ & $\begin{array}{c}\text { Energie } \\
{[\mathrm{keV}]}\end{array}$ & $\begin{array}{c}\text { Fluenz } \\
{\left[\mathrm{cm}^{-2}\right]}\end{array}$ & $\begin{array}{c}\text { Eindringtiefe } \\
{[\mathrm{nm}]}\end{array}$ \\
\hline Mangan & 55 & 250 & $2 \cdot 10^{15}$ & 104 \\
Gadolinium & 157 & 300 & $10^{14}$ & 54 \\
Silizium & 29 & 60 & $10^{14}$ & 46 \\
\hline
\end{tabular}

Tabelle 4.3: Implantationsparameter zur Erzeugung der Referenzproben für SIMS-Messungen und daraus mittels TRIM berechnete Eindringtiefe.

versteht man den Effekt, dass die Ionen bei Eintritt in die Probe entlang niedrig indizierter Achsen (wie etwa der [0001]-Achse im Fall von GaN) „Kanäle“ finden können. Innerhalb dieser Kanäle befinden sich keine Atome. Trifft ein Ion unter einem Winkel von $\lesssim 5^{\circ}$ in einen solchen Kanal, so führt es ausschließlich Kleinwinkelstreuungen mit den Atomen in den „Wänden“ des Kanals aus, die einen fokussierenden Effekt haben. Das Ion dringt dann viel weiter ein, als es im so genannten ,random"-Fall passieren würde, bei dem die Atome zufällig verteilt auf dem Pfad des Ions liegen und auf dem die Simulationen beruhen. Dieser Effekt kann aber auch vorsätzlich herbeigeführt werden, um höhere Implantationstiefen bei geringerem Schaden in der Matrix zu erreichen.

Eine weitere Anwendung des Channeling-Effekts ist das RBS-Channeling, kurz RBS/C. Beim Standard-RBS wird die Probe ebenfalls in ,random"-Konfiguration montiert, beim RBS/C erhält man ein völlig anderes Spektrum. In einer idealen Probe trägt nun nur noch die oberste Monolage zum RBS-Signal bei, da alle darunter liegenden Atomkerne abgedeckt sind. Das RBS-Signal für jedes Element fällt also nach einem schmalen Signal an der Oberfläche sofort wieder ab. In einem realen Kristall wird dieser Effekt durch Defekte wie Zwischengitteratome, Versetzungen oder Stapelfehler abgeschwächt, so dass das Rest-Signal als Maß für die Kristallqualität benutzt werden kann. Da außerdem elementspezifisch gemessen wird, können Aussagen darüber gemacht werden, auf welchem Gitterplatz ein Fremdatom eingebaut wurde. Zeigt sein Signal das gleiche Verhalten wie ein Matrix-Atom, kann von einem substitutionellen Einbau ausgegangen werden. Zeigt sich ein deutlich erhöhtes Signal in Channeling-Konfiguration, deutet dies auf einen interstitiellen Einbau in genau diesem Kanal hin.

\subsubsection{Sekundär-Ionen Massenspektroskopie (SIMS)}

Sekundär-Ionen Massenspektroskopie (SIMS) ist eine Methode, bei der die nach z. B. Teilchenbeschuss emittierten Ionen mittels Massenspektroskopie analysiert werden. Die Probe wird einem Primär-Ionenstrahl ausgesetzt, der Energien im Bereich $1-25 \mathrm{keV}$ besitzt. Die auf die Oberfläche auftreffenden Ionen verlieren ihre Energie innerhalb weniger Nanometer (vergleiche auch Implantation, Kapitel 4.3.2). Durch die Kollisionskaskaden wird diese Energie teilweise an Atome der obersten Monolage abgegeben. Wenn diese Energie ausreicht, um das Atom aus der Schicht herauszulösen, spricht man von einem Sputterprozess. Die meisten dieser gesputterten Atome sind elektrisch neutral, ein gewisser Prozentsatz liegt allerdings in ionisierter Form vor, den so genannten SekundärIonen. Diese haben nur eine geringe kinetische Energie (einige $10 \mathrm{eV}$ ) und werden durch 
ein elektrisches Feld nachbeschleunigt. Danach werden sie durch ein Massenspektrometer geleitet und detektiert. Die detektierten Massen geben Aufschluss über die elementare Zusammensetzung der Schicht und können Konzentrationen typischerweise im ppm-Bereich zuverlässig detektieren. Der verwendete Aufbau ist in Abbildung 4.8 dargestellt.

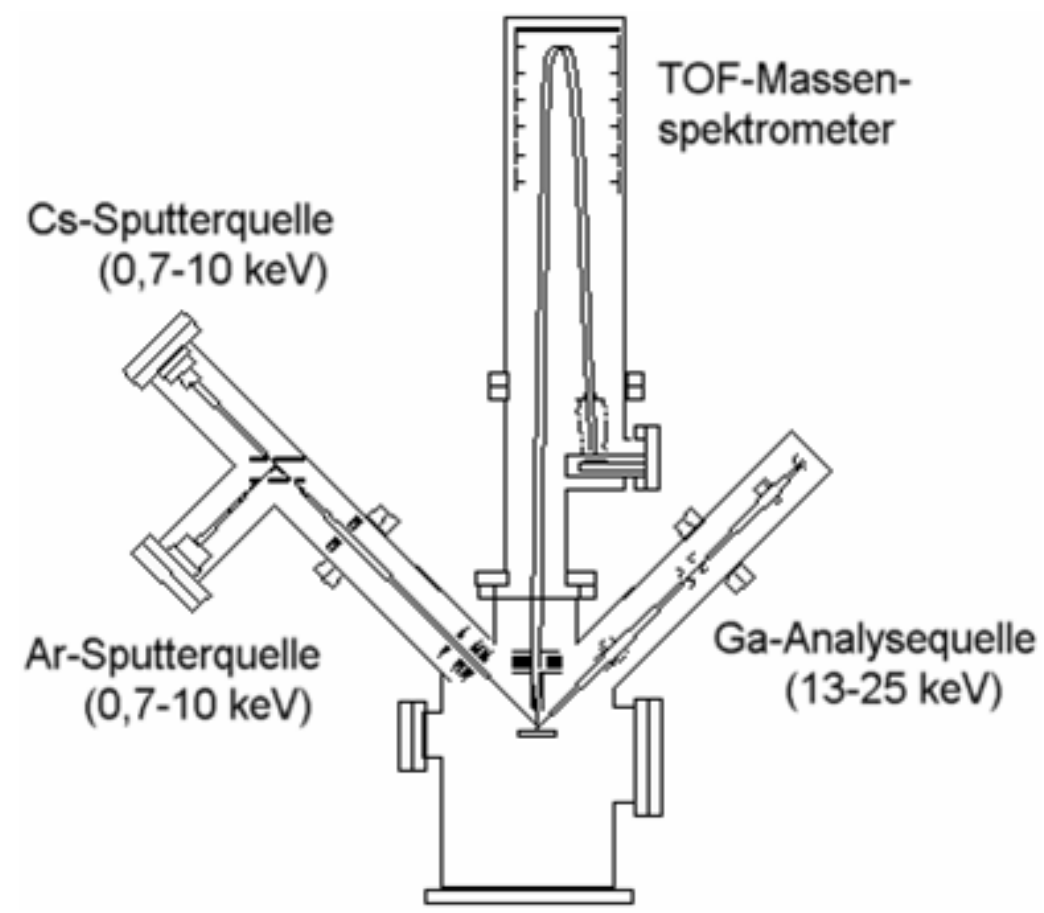

Abbildung 4.8: Schematischer Aufbau des verwendeten TOFSIMS-Systems.

Das eingesetzte Massenspektrometer arbeitet nach der „time of flight"-Methode (kurz TOF). Die Sekundär-Ionen werden dabei auf eine Energie $V$ im $k e V$-Bereich beschleunigt und auf eine feldfreie Strecke der Länge $L$ geleitet. Die benötigte Flugzeit berechnet sich nach

$$
t=L \sqrt{\frac{m}{2 q V}},
$$

wobei $m$ die Masse und $q$ die Ladung der entsprechenden Ionen ist. Es ist also ein extrem kurz gepulster Primärstrahl nötig, um eine hohe Massenauflösung zu erreichen. Unterschiede in der Flugzeit durch verschiedene Startenergien nach dem Sputterprozess werden effektiv durch einen Ionenspiegel ausgeglichen. Das zeitabhängige Detektorsignal kann dann nach Gleichung 4.22 in eine $m / q$-Skala umgerechnet werden. Dabei müssen Masseninterferenzen berücksichtigt werden, wie sie beispielsweise zwischen ${ }^{56} \mathrm{Fe}^{2+}$ und ${ }^{28} \mathrm{Si}^{+}$ auftreten oder zwischen ${ }^{156} \mathrm{Gd}^{+}$und $\left({ }^{71} \mathrm{Ga}_{2}{ }^{14} \mathrm{~N}\right)^{+}$. In den vorliegenden Fällen konnten diese entweder durch die hohe Massenaufösung des TOFSIMS direkt unterschieden werden oder durch die Einschränkung auf geeignete Isotope (wie ${ }^{160} \mathrm{Gd}^{+}$) umgangen werden. Der Primärstrahl für die Analyse bestand aus ${ }^{69} \mathrm{Ga}$, so dass für die GaN-Schichten nur das ${ }^{71} \mathrm{Ga}$-Signal analysiert wurde. Die Energie des Primärstrahls war $25 \mathrm{keV}$ bei einem Strom (pro Puls) von $0.02 p A$ und einer Fläche von $200 \times 200 \mu m^{2}$. Um ein Tiefenprofil 
zu erhalten wurde mit einer zweiten Sputterquelle zwischen den einzelnen Analysepulsen die Schicht abgetragen. Dies wurde mit einem $2 \mathrm{keV}$-Ar-Strahl erreicht, der mit $15 \mathrm{nA}$ auf eine Fläche von $500 \times 500 \mu \mathrm{m}^{2}$ fokussiert wurde.

Ein kritischer Parameter bei der SIMS-Analyse der verschiedenen Elemente ist der Anteil der ionisierten Atome pro gesputtertem Atom. Dieser variiert um mehrere Größenordnungen und liegt im Bereich $10^{-6}-10^{-2}$. Im Falle positiver Ionen, wie sie bei den meisten Metallen vorliegen, kann er oft durch Oxidierung der Oberfläche stark erhöht werden. Für die analysierten Metalle Ga, Mn und Gd traf dies zu, so dass alle Messungen unter einem Sauerstoffpartialdruck von $10^{-6}$ mbar durchgeführt wurden, so dass eine Oxidation der Oberfläche gewährleistet war.

Zur quantitativen Analyse der Tiefenprofile wurden Referenzproben implantiert (siehe Kapitel 4.3.2). Diese weisen ein durch Simulationen sehr gut bekanntes Tiefenprofil auf. Anhand eines Vergleichs dieser Simulationen mit einer SIMS-Messung an der implantierten Probe können die Längen- und die Konzentrations-Achse der SIMS-Profile kalibriert werden. Bei sehr scharfen Dotierprofilen tritt in den SIMS-Messungen der Effekt des Ionenmischens zu Tage. Er entsteht, weil beim Sputterprozess ein Teil der Schichtatome durch die Kollisionskaskaden in eine größere Tiefe gedrückt wird. Grenzflächen erscheinen daher in den SIMS-Profilen nicht als atomar scharf sondern etwas verschmiert. Dieser Effekt muss bei der Analyse der Homogenität des Fremdatom-Einbaus berücksichtigt werden.

\subsubsection{Röntgen-Diffraktometrie (XRD)}

Zur strukturellen Analyse wurden XRD-Messungen (Röntgenbeugung) an den gewachsenen Schichten durchgeführt. Die benutzte Anlage war ein Vollschutzgerät vom Typ D5000 von Siemens (jetzt Bruker) mit Cu-Anode. Die Wellenlänge dieses Röntgenquellen-Typs beträgt $\lambda=1.54056 \AA$ für die $K_{\alpha 1}$-Linie. Wann immer nötig, wurde die ebenfalls im Spektrum auftretende $K_{\alpha 2}$-Linie durch eine geeignete Anpassung während der Datenanalyse herausgerechnet. Die Geometrie des Versuchsaufbaus ist in Abbildung $4.9 \mathrm{zu}$ sehen.

Der Winkel der Probenoberfläche relativ zum einfallenden Röntgenstrahl wird mit $\omega$ bezeichnet, der des Detektors mit 2 $\theta$. Es wurden Messungen in $\theta$-2 $\theta$-Geometrie durchgeführt um mögliche Ausscheidungen zu detektieren. Dabei wird der Probenwinkel $\omega$ fest mit dem Detektorwinkel $2 \theta$ gekoppelt im Verhältnis $\omega=\frac{2 \theta}{2}$, wie auch in Abbildung 4.9 dargestellt. Damit erreicht man einen symmetrischen Strahlengang und nur Gitterebenen, die parallel zur Probenoberfläche liegen, können ein Signal geben. Da beim epitaktischen Wachstum immer eine Textur des gewachsenen Films vorliegt, kann davon ausgegangen werden, dass auch Ausscheidungen mit unbekannter kristalliner Struktur hier einen Reflex liefern. Die erhaltenen Spektren liefern Information über die Gitterkonstanten der untersuchten Materialien. Im Falle von GaN mit Wachstumsrichtung [0001] ergibt sich die Gitterkonstante $c$ aus dem Signal beim Winkel $2 \theta$ nach:

$$
c=\frac{n \lambda}{\sin \theta}
$$

Dabei ist $\lambda$ die Wellenlänge der verwendeten Röntgenstrahlung und $n$ die Ordnung des Reflexes. Mit Hilfe der bekannten Werte für Volumen-GaN lassen sich so auch Verspannungen im Material analysieren. Die Breite der jeweiligen Signale kann als Maß für die 


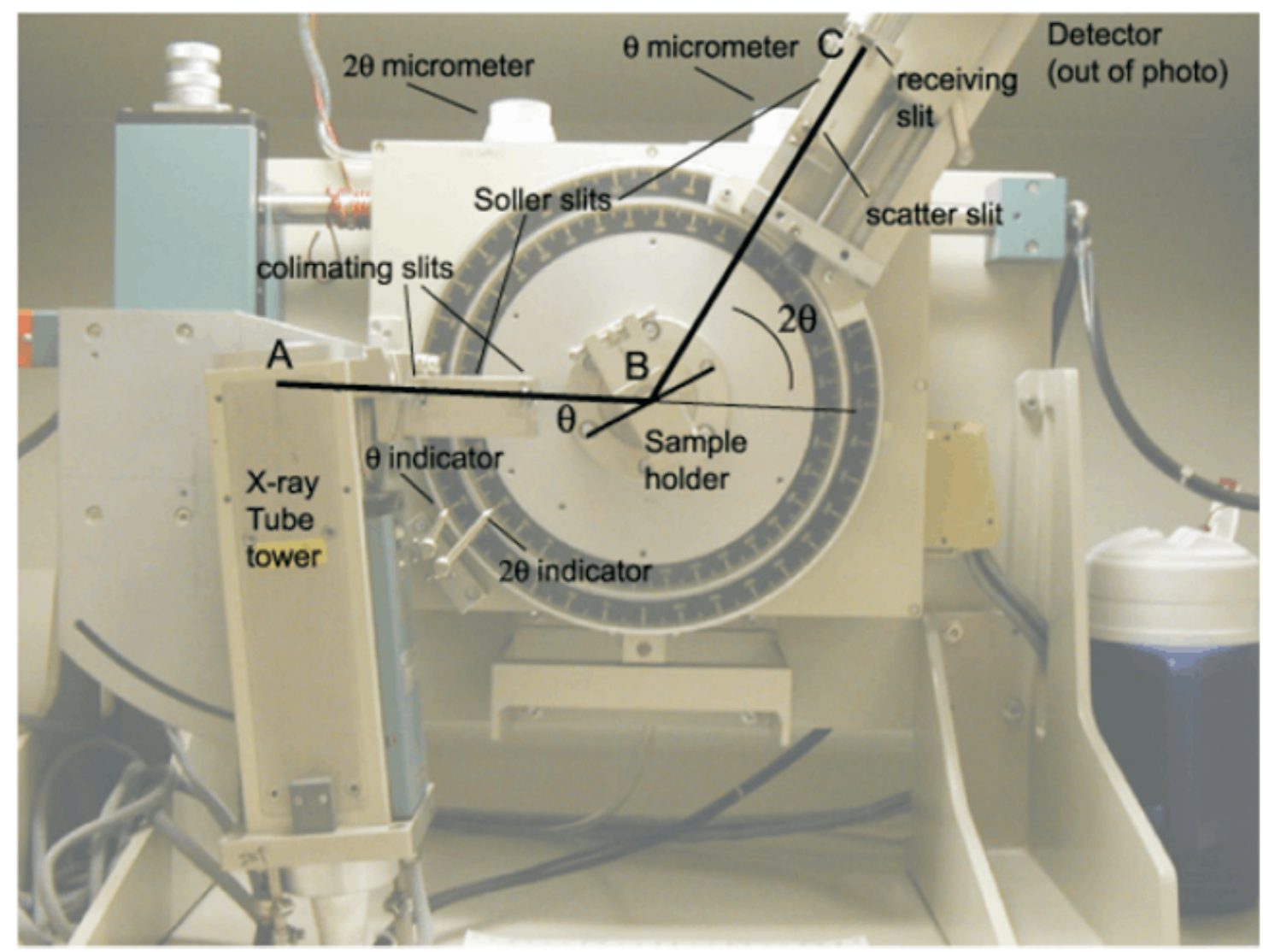

Abbildung 4.9: Schematischer Aufbau des XRD-Gerätes.

Qualität einer Schicht benutzt werden, da sie letztendlich die statistische Verteilung der Gitterkonstanten über die Dicke der Schicht angibt. Bei hoher Kristallqualität mit wenigen ausgedehnten Defekten ergibt sich eine schmale Verteilung, eine hohe Anzahl an Defekten verursacht typischerweise eine Verbreiterung des Signals.

Die zweite verwendete Geometrie ermöglichte das Aufnehmen so genannter „rocking curves". Hierbei wird der Detektorwinkel $2 \theta$ festgehalten und der Probenwinkel $\omega$ variiert. Diese Technik wird bei einem bestimmten Reflex aus der $\theta$-2 $\theta$-Messung angewandt, um zusätzliche Informationen zu erhalten. Diese bezieht sich auf die Winkelverteilung der c-Achse im Falle von GaN. Da das Wachstum, je nach Wachstumsbedingungen, häufig zu so genannten mosaik-artigen Strukturen führt, können die einzelnen Mosaike leicht voneinander abweichende Orientierungen aufweisen. Dadurch wird auch bei Verkippung der Probe noch ein Teil davon ein detektierbares Signal verursachen. Das Signal ist auf Grund der statistischen Verteilung nahezu Gauss-förmig. Die Breite wird als ein wesentliches Kriterium bei der Bestimmung der Kristallqualität eingesetzt. 


\section{Struktur und Phasen bei verdünnten GaMnN-Schichten}

Beim Wachstum dünner Schichten auf Heterosubstraten spielen viele Parameter eine Rolle, wie in Kapitel 3 dargelegt. Bevor daher tiefer gehende Analysen der optischen und magnetischen Eigenschaften möglich sind, ist eine genaue strukturelle Charakterisierung notwendig, um festzustellen, ob alle Wachstumsprozesse wie geplant abgelaufen sind.

\subsection{Bildung einer zweiten Phase}

Der Wachstumsprozess zielt darauf, GaN herzustellen, in dem in zufälliger Verteilung einige Prozent der Ga-Atome durch Mn-Atome ersetzt werden. Der sich dadurch ergebende verdünnte magnetische Halbleiter wird GaMnN genannt. Allerdings gibt es im ternären System Ga-Mn-N weitere stabile Phasen. So findet man verschiedene geordnete $\mathrm{Ga}_{x} \mathrm{Mn}_{y^{-}}$ Legierungen mit fester Zusammensetzung oder die in Perowskit-Struktur kristallisierenden Phasen $\mathrm{Ga}_{x} \mathrm{Mn}_{4-x} \mathrm{~N}(0<x<1)$ mit variablem Ga-Anteil. Die verwendete Wachstumsmethode der MBE findet nicht im thermodynamischen Gleichgewicht statt, so dass es schwer fällt, hierbei eine Vorhersage zu machen, welche Phasen auftreten werden. Daher können nur experimentelle Untersuchungen die Erkenntnis liefern, ob das Wachstum ohne Ausscheidungen ablief.

\subsubsection{Nachweis im XRD}

Die Standard-Methode zum Auffinden von Ausscheidungen ist XRD mit einer $\theta-2 \theta$ Messung (siehe Kapitel 4.3.4). Abbildung 5.1 zeigt beispielhaft das Ergebnis einer solchen Messung an Probe J0087 bei der verschiedene Signale sichtbar werden. Diese werden nach ihrer Ursache gruppiert in Tabelle 5.1 zusammengefasst.

Die Signale lassen sich im Rahmen der Messgenauigkeit dieser Übersichtsmessung $\left(0.1^{\circ}\right)$ den angegebenen Reflexen zuordnen. Die Abweichung der Signale vom Al-Probenhalter ist mit $0.3^{\circ}$ etwas größer. Es handelt sich hierbei um einen systematischen Fehler, der durch die tiefere Lage des Probenhalters im Vergleich zur Schicht verursacht wird. Der symmetrische $\theta$-2 $\theta$-Strahlengang ist dann nicht genau erfüllt und alle Signale werden verschoben.

Die Position der drei Signale der unbekannten zweiten Phase in der GaMnN-Schicht geben schon einen ersten Hinweis auf deren Kristallstruktur. Das Signal bei $86.9^{\circ}$ kann eindeutig der 2. Ordnung des Signals bei $40.3^{\circ}$ zugeordnet werden, da es exakt dem halben Netzebenenabstand entspricht. Berechnet man weiterhin die beiden Netzebenenabstände 


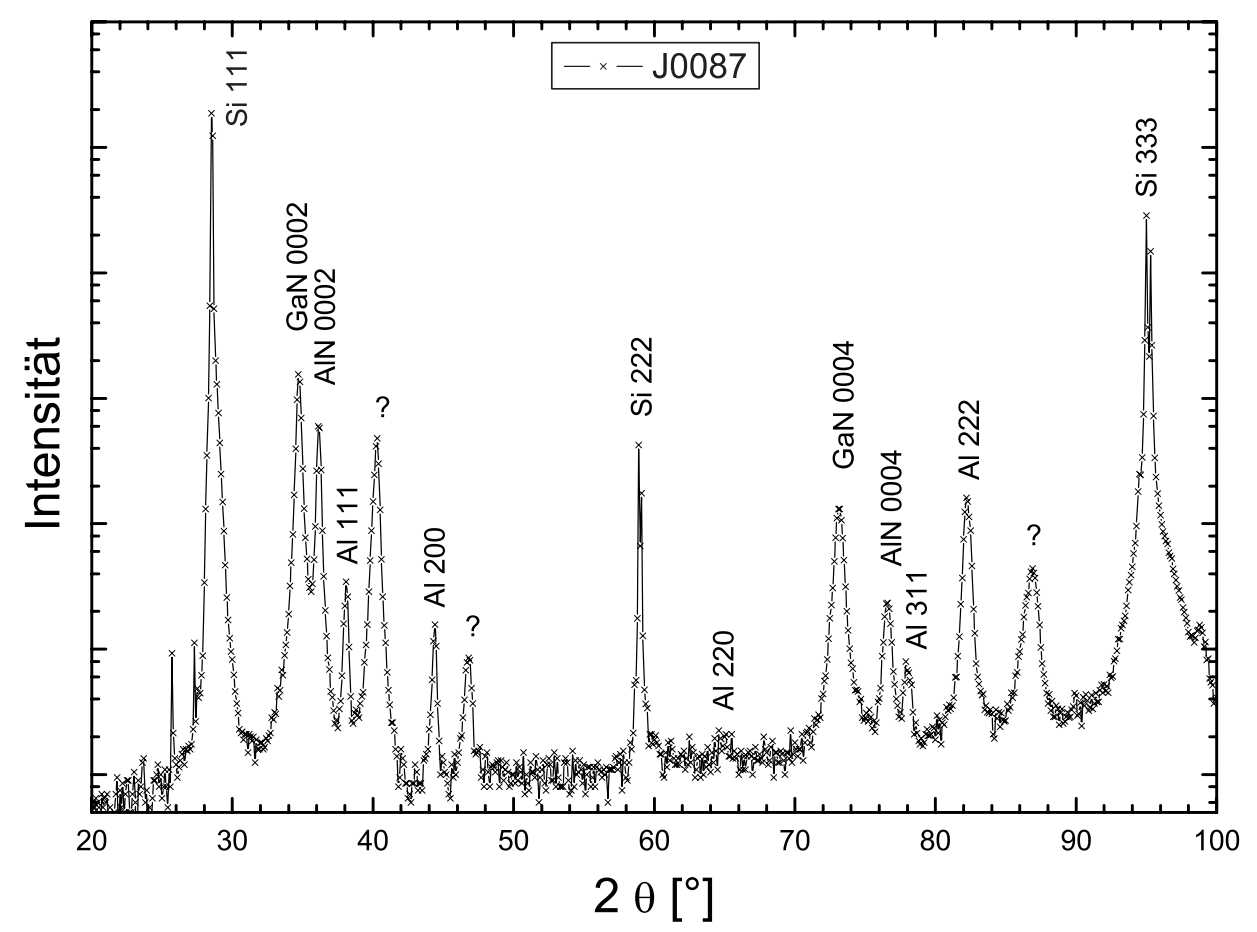

Abbildung 5.1: XRD-Messung an einer Probe mit hohem Mn-Angebot. Man erkennt Signale vom Si-Substrat, dem AlN-Puffer, der GaN-Schicht und dem Al-Probenhalter. Es bleiben drei nichtzugeordnete Signale übrig (,?"), die von einer zweiten Phase in der Schicht stammen müssen.

\begin{tabular}{|l||c|c|}
\hline Phase & Winkel 2 $\theta\left[^{\circ}\right]$ & Reflex \\
\hline \hline \multirow{2}{*}{ Al (Probenhalter) } & 38.1 & $(111)$ \\
& 44.4 & $(200)$ \\
& 65.0 & $(220)$ \\
& 77.9 & $(311)$ \\
& 82.2 & $(222)$ \\
\hline Si (Substrat) & 28.5 & $(111)$ \\
& 58.9 & $(222)$ \\
& 95.0 & $(333)$ \\
\hline AlN (Puffer) & 36.1 & $(0002)$ \\
& 76.6 & $(0004)$ \\
\hline GaN bzw. GaMnN & 34.7 & $(0002)$ \\
& 73.1 & $(0004)$ \\
\hline unbekannt & $40.266 \pm 0.003$ & R1 \\
& $46.78 \pm 0.01$ & R2 \\
& $86.87 \pm 0.01$ & R1 (2. Ordnung) \\
\hline
\end{tabular}

Tabelle 5.1: Röntgenreflexe in Probe J0087 mit 32.6\% Mn-Gehalt bei einer $\theta$-2 $\theta$-Messung (Abbildung $5.1)$.

$d$ für R1 und R2, so ergibt sich folgendes Verhältnis:

$$
\left(\frac{d(R 1)}{d(R 2)}\right)^{2}=\left(\frac{2.2397 \AA}{1.9419 \AA}\right)^{2}=1.330 \approx 4: 3 .
$$


Ein Verhältnis von 4:3 würde exakt dem von (200) und (111) einer kubischen Struktur entsprechen. Die kleine Abweichung (0.23\%) von diesem Wert kann durch Verspannungen erklärt werden, denen die Ausscheidungen durch das umgebende GaMnN unterworfen sind. Es ergibt sich damit eine Gitterkonstante von 3.88 A. Diese Aussagen gelten allerdings nur unter der Annahme, dass es genau eine zweite Phase in der Schicht gibt, theoretisch könnte es auch mehrere verschiedenartige Ausscheidungen geben. Es wird im Folgenden noch gezeigt werden, dass es sich nur um eine Art von kubischen Ausscheidungen handelt und die Zuordnung der Reflexe korrekt ist, so dass diese Bezeichnung ab jetzt verwendet werden wird.

Der Intensitätsunterschied der (200)- und (111)-Signale von nahezu zwei Größenordnungen deutet auf eine starke Texturierung hin. Bei einem perfekten epitaktischen Wachstum würde man eine vollständige Texturierung der Schicht erwarten, wie es beispielsweise beim GaMnN zu beobachten ist. Hier ist nur die [0001]-Richtung durch die entsprechenden Reflexe sichtbar. Der Großteil der Ausscheidungen wächst mit seiner [111]-Richtung parallel dazu. Dies ist in Übereinstimmung mit der Tatsache, dass bei kubisch bzw. hexagonal dichtesten Packungen die (111)- bzw. (0001)-Ebenen äquivalent sind, so dass diese Orientierung bevorzugt werden sollte.

Um die zweite Phase zu identifizieren, wurden alle in der Datenbank PCPDFWIN [97] dokumentierten Verbindungen des ternären Systems Ga-Mn-N überprüft. Alle dort aufgeführten GaMn-Legierungen und elementaren Modifikationen von Ga und Mn kommen nicht in Frage, ebensowenig wie die meisten Mn-N-Verbindungen, da deren Signale nicht in Übereinstimmung mit den vorliegenden Messungen zu bringen sind. Es finden sich aber zwei Verbindungen, $\mathrm{Mn}_{4} \mathrm{~N}$ und $\mathrm{GaMn}_{3} \mathrm{~N}_{0.5}$, die zu der unbekannten zweiten Phase passen würden. Deren Reflexe sind in Tabelle 5.2 aufgeführt. Es sind beides kubische Phasen mit Gitterkonstanten von $3.846 \AA$ und $3.898 \AA$.

\begin{tabular}{|c||c|c|c|c|}
\hline \multicolumn{1}{|c||}{ Reflex } & \multicolumn{2}{c|}{$\mathbf{M n}_{4} \mathbf{N}$} & \multicolumn{2}{c|}{$\mathbf{G a M n}_{3} \mathbf{N}_{0.5}$} \\
& $2 \theta\left[^{\circ}\right]$ & Intensität & $2 \theta\left[{ }^{\circ}\right]$ & Intensität \\
\hline$(110)$ & - & & 32.488 & 3 \\
$(111)$ & 40.450 & 100 & 40.056 & 100 \\
$(200)$ & 47.347 & 66 & 46.600 & 50 \\
$(220)$ & 69.062 & 27 & 68.035 & 20 \\
$(311)$ & 83.301 & 27 & 82.007 & 16 \\
$(222)$ & 87.978 & 3 & 86.512 & 4 \\
\hline
\end{tabular}

Tabelle 5.2: Röntgenreflexe von $\mathrm{Mn}_{4} \mathrm{~N}$ und $\mathrm{GaMn}_{3} \mathrm{~N}_{0.5}$ nach [97]. Die Intensitäten beziehen sich auf Pulverproben und sind auf den stärksten Reflex normiert.

Eine eindeutige Identifizierung der Ausscheidungen ist zwingend erforderlich, da diese die magnetischen Eigenschaften der Proben mit bestimmen können. Auch in Proben ohne charakteristische Signale einer zweiten Phase im XRD könnte diese in Form von NanoKristalliten vorhanden sein, die unterhalb der Nachweisempfindlichkeit liegen. Daher ist die Kenntnis der Ausscheidung wichtig, die sich bei gegebenen Wachstumsparametern als thermodynamisch am günstigsten herausstellt, um magnetische Messungen interpretieren zu können unter dem Gesichtspunkt, dass auch Ausscheidungen für die Signale 
verantwortlich sein könnten.

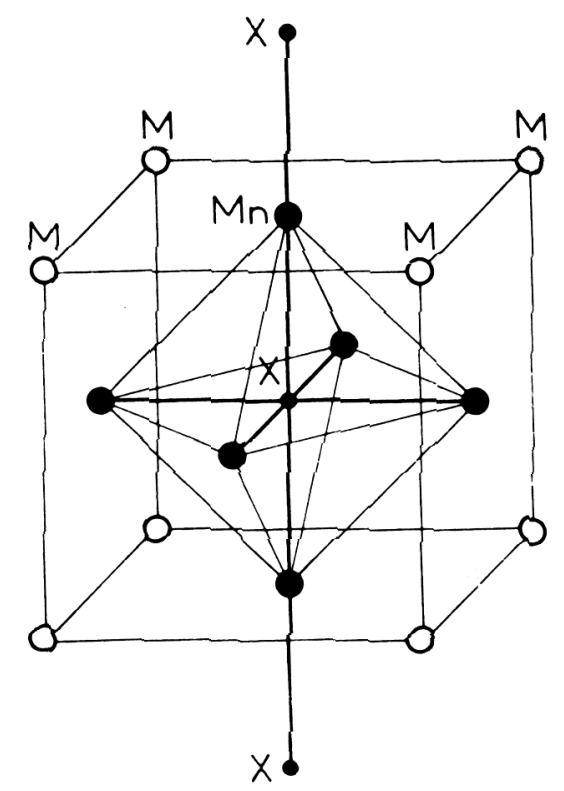

Abbildung 5.2: Struktur von $\left(\mathrm{Mn}_{1-x} \mathrm{Ga}_{x}\right) \mathrm{Mn}_{3} \mathrm{~N}$ nach [98]. X bezeichnet den Stickstoff, die Position $\mathrm{M}$ wird wahlweise von Mn oder Ga besetzt.

In der Literatur ist vom System $\left(\mathrm{Mn}_{1-x} \mathrm{Ga}_{x}\right) \mathrm{Mn}_{3} \mathrm{~N}$ bekannt, dass es als PerowskitStruktur kristallisiert [98]. Die Kristallstruktur ist in Abbildung $5.2 \mathrm{zu}$ sehen. Es gibt zwei nicht-äquivalente Untergitter auf denen sich die Metall-Atome befinden und das Stickstoff-Untergitter. Dieses muss allerdings nicht vollständig besetzt sein, so berichtet Bouchaud [99] über $\mathrm{Mn}_{4} \mathrm{~N}_{y}$ mit $0.7<y<1.2$, wo der Stickstoff die 4 Oktaederlücken des Mn-fcc-Gitters mit entsprechender Stöchiometrie besetzt. Im Falle von $\mathrm{GaMn}_{3} \mathrm{~N}_{0.5}$ ist das N-Untergitter nur halb besetzt.

Die Position der Signale der Ausscheidung variiert zwischen verschiedenen Proben geringfügig, im Mittel ergibt sich ein Wert von $39.96 \pm 0.07^{\circ}$ für den (111)-Reflex und $46.59 \pm 0.03^{\circ}$ für den (200)-Reflex. Dies ergibt Gitterkonstanten von $3.905 \pm 0.007 \AA$ bzw. $3.896 \pm 0.003 \AA$. Die Gitterkonstanten im System $\left(\mathrm{Mn}_{1-x} \mathrm{Ga}_{x}\right) \mathrm{Mn}_{3} \mathrm{~N}_{y}$ steigen mit höherem $x$ und $y$ an $[98,99]$ und erreicht für $x=y=1$ etwa $3.896 \AA$. Das deutet darauf hin, dass es sich bei den Ausscheidungen um $\mathrm{GaMn}_{3} \mathrm{~N}$ handelt. Allerdings können auch Verspannungen eine Rolle spielen, so dass diese Zuordnung noch nicht eindeutig ist.

Abbildung 5.3 zeigt eine $\theta$-20-Messung an Probe J0121 mit 14.6\% Mn-Gehalt. Hier wie auch in wenigen anderen Proben ist zusätzlich zum Hauptsignal der Ausscheidung noch eine Schulter bei größeren Winkeln (entsprechend einer etwas kleineren Gitterkonstante) zu finden. Das deutet darauf hin, dass je nach Wachstumsbedingungen der Probe verschiedene Phasen im System $\left(\mathrm{Mn}_{1-x} \mathrm{Ga}_{x}\right) \mathrm{Mn}_{3} \mathrm{~N}_{y}$ stabil sein könnten. Auch die Abweichungen des Hauptsignals von $39.748^{\circ}$ (Probe J0125) bis $40.266^{\circ}$ (Probe J0087) sind möglicherweise nicht auf Verspannungen zurückzuführen, sondern auf eine unterschiedliche Stöchiometrie. Diese sollte aber zumindest ungefähr $\mathrm{GaMn}_{3} \mathrm{~N}$ entsprechen. Eine eindeutige Aussage hierzu können $\theta$-2 $\theta$-Messungen alleine nicht liefern. 


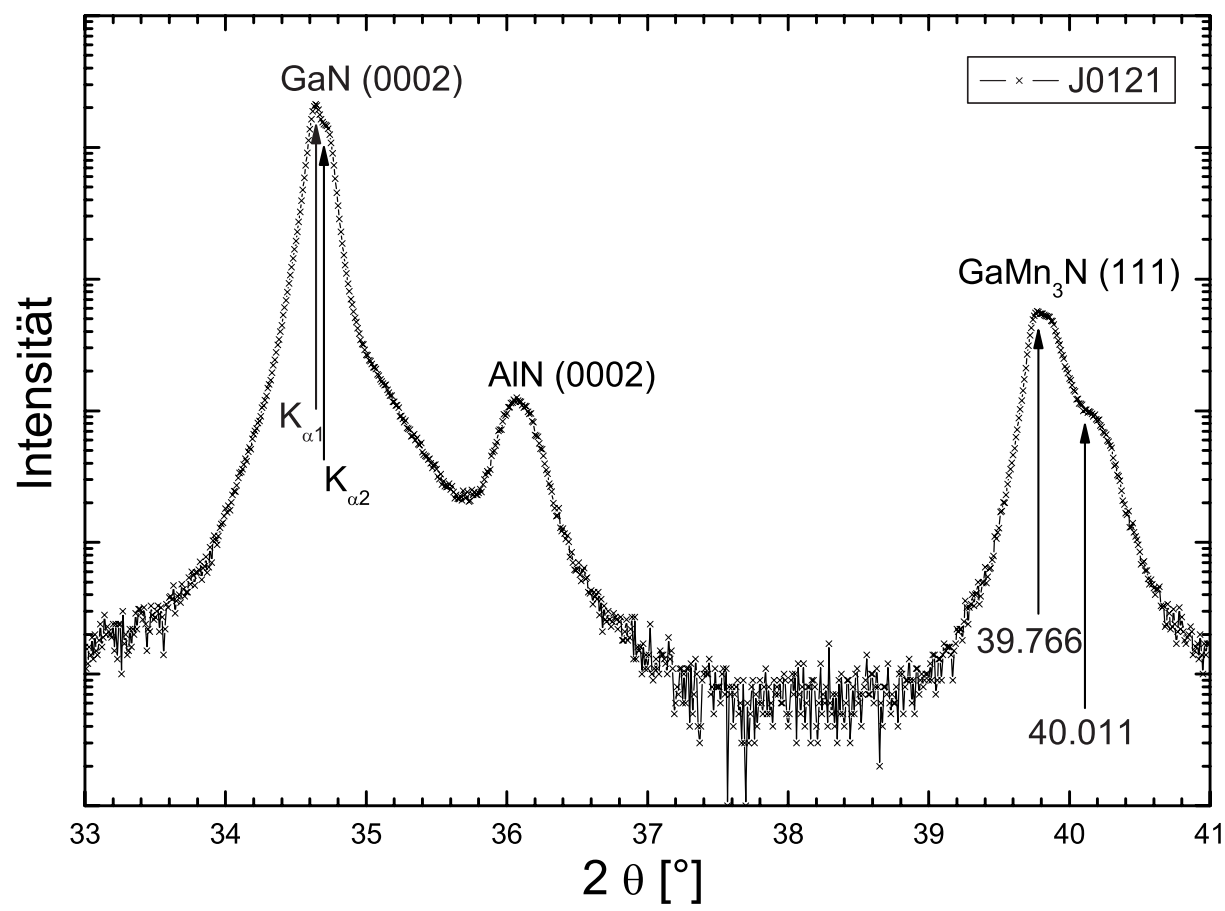

Abbildung 5.3: Hochaufösende XRD-Messung an Probe J0121 mit 14.6 \% Mn-Gehalt. Man erkennt die $\mathrm{K}_{\alpha 1^{-}}$und $\mathrm{K}_{\alpha 2}$-Linie bei GaN (0002). Außerdem sind im Bereich der Ausscheidung zwei verschiedene Signale auflösbar.

Vergleichbare Ausscheidungen finden sich auch in der veröffentlichten Literatur. Meist wird die Ausscheidung dabei $\mathrm{GaMn}_{3} \mathrm{~N}$ zugeordnet [100, 101, 102, 103, 104, 105, 106], es findet sich aber auch $\mathrm{GaMn}_{3} \mathrm{~N}_{0} .5$ [107], $\mathrm{Mn}_{4} \mathrm{~N}$ [108] sowie verschiedene andere Mn-NVerbindungen [109, 110]. Letztere liefern allerdings eindeutige Röntgensignale und sind daher unkritisch. Zusätzlich zu den hier meist verwendeten XRD-Messungen werden dabei manchmal Argumente bezüglich der magnetischen Eigenschaften [99, 111, 98, 112] dieser Ausscheidungen benutzt um die verschiedenen $\left(\mathrm{Mn}_{1-x} \mathrm{Ga}_{x}\right) \mathrm{Mn}_{3} \mathrm{~N}_{y}$-Phasen zu identifizieren. Da allerdings die magnetischen Eigenschaften von GaMnN gerade Ziel dieser Forschung und nicht bekannt ist, ist diese Vorgehensweise mit Vorsicht zu genießen. Eine klare Identifikation liefern aber Giraud et al. [106], die mittels EXAFS die lokale Umgebung der Mn-Atome messen können und dabei durch Simulationen eine gute Übereinstimmung mit $\mathrm{GaMn}_{3} \mathrm{~N}$ und nicht $\mathrm{Mn}_{4} \mathrm{~N}$ erreichen.

\subsubsection{Identifikation im TEM}

Zur endgültigen Identifizierung der Ausscheidungen wurden im Rahmen der Doktorarbeit von Tore Niermann [113] lokale EDX-Messungen im TEM durchgeführt. Damit kann auf der Nanometerskala die elementare Zusammensetzung in der Probe bestimmt werden. Abbildung 5.4 zeigt das Ergebnis dieser Untersuchungen an Probe J0121. Das Elementverhältnis von Ga:Mn ist innerhalb der Ausscheidung nahezu perfekt 1:3.

Für diese Probe ist damit klar, dass die Ausscheidungen einer $\mathrm{GaMn}_{3} \mathrm{~N}$-Phase zuzu- 
(a)

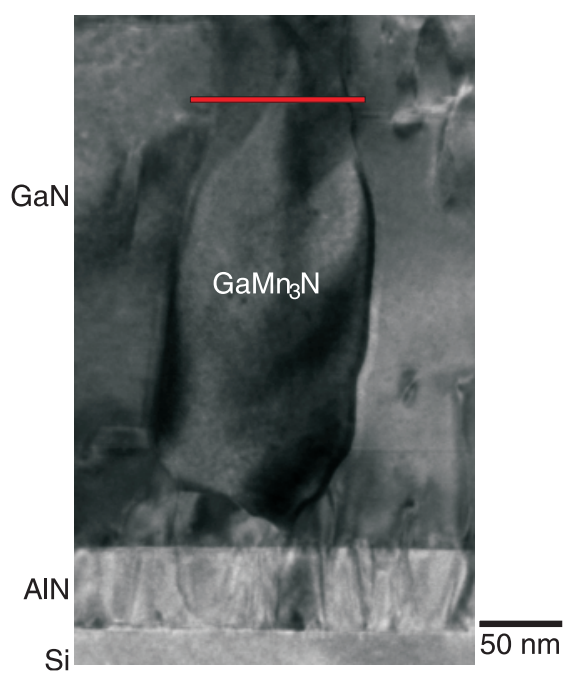

(b)

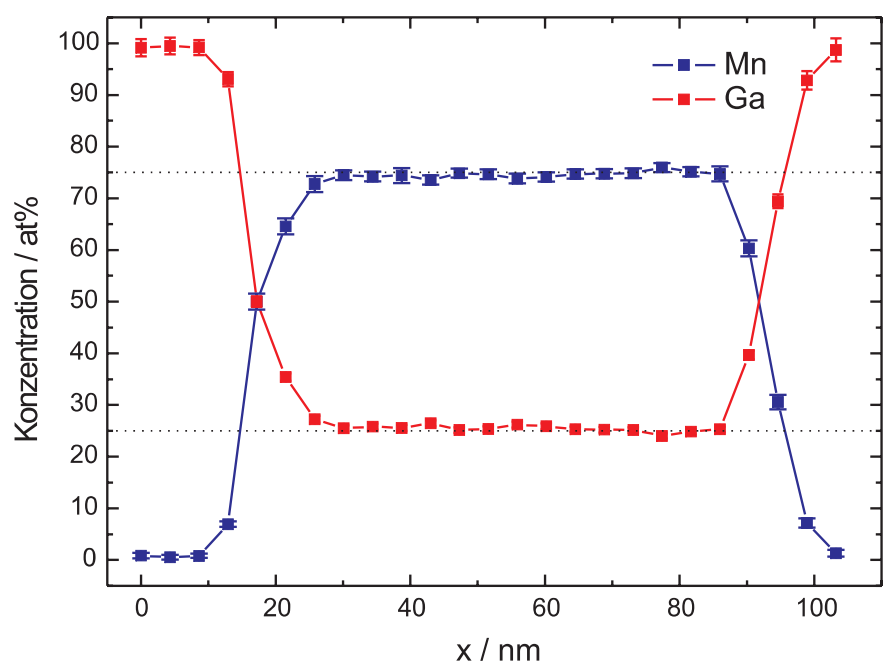

Abbildung 5.4: EDX-Analyse einer Ausscheidung in Probe J0121 nach [113]. (a) TEM-Aufnahme der Ausscheidung. Die EDX-Messung wurde entlang der roten Linie durchgeführt. (b) Relatives Verhältnis von Mn- und Ga-Konzentration. Innerhalb der Ausscheidung ergibt sich im Rahmen der Messgenauigkeit ein Verhältnis von Ga:Mn = 1:3.

ordnen sind. Dies war bereits anhand der Gitterkonstanten abschätzbar, die aus XRDMessungen bestimmt wurde, da sie im oberen Bereich aller Proben lag. Probe J0087, die die kleinste Gitterkonstante bei den Ausscheidungen aufweist wurde ebenfalls mittels EDX im TEM untersucht. Das Ergebnis zeigt auch hier relative Mn-Konzentrationen von etwa 75\%. Daher wird im Folgenden davon ausgegangen, dass in allen vorliegenden Proben die Ausscheidungen in Form von $\mathrm{GaMn}_{3} \mathrm{~N}$ vorliegen.

\subsubsection{Wachstum ohne Ausscheidungen}

Das wesentliche Ziel des Wachstums war die Herstellung von GaMnN-Schichten, die eine einzige Phase ohne Ausscheidungen aufwiesen. Um dies zu erreichen, wurden verschiedene Parameter des Wachstumsprozesses variiert, um ein „Fenster“ zu identifizieren, bei dem dies möglich ist. Wie im Rahmen der Diplomarbeit von Martin Röver [56] ermittelt wurde, muss dabei zuerst ein kontrollierbarer und reproduzierbarer Einbau von Mn in die Schichten gewährleistet sein. Es stellt sich heraus, dass bei hohen Substrattemperaturen während des Wachstums auf $\mathrm{Si}(111)$ dieses Ziel nicht erreicht werden kann. Erst bei einem Absenken der Wachstumstemperatur von $760^{\circ} \mathrm{C}$ auf $650^{\circ} \mathrm{C}$ ist dies möglich. Im Bereich des Wachstums ohne Ausscheidungen ergibt sich eine Einbau-Effizienz von $27 \%$ [66]. Das bedeutet, dass die Mn-Konzentration in der Schicht nach dem Wachstum $27 \%$ des angebotenen Mn:Ga-Verhältnisses beträgt.

Abbildung 5.5a) zeigt den Einfluss des Mn-Angebots. Während Probe J0093 (gewachsen bei $650^{\circ} \mathrm{C}$ mit einem Gesamtmetall-Fluss von $1 \times 10^{-8} \mathrm{mbar}$ und $10 \%$ relativem MnAngebot) noch frei von Ausscheidungen ist, werden bei höherem Mn-Angebot Ausscheidungen sichtbar. Bei einem geringen Anteil an Ausscheidungen sind diese bevorzugt in 

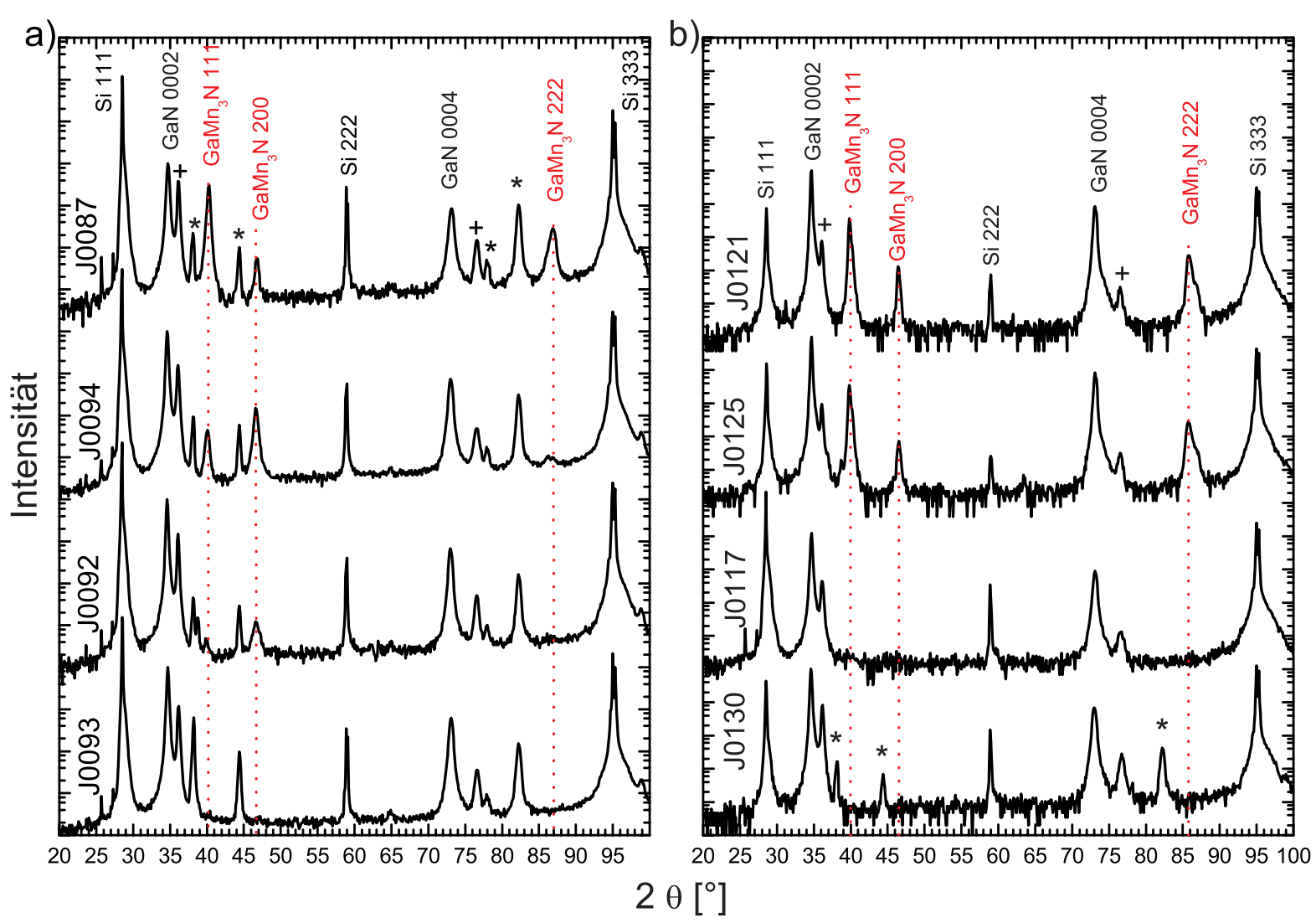

Abbildung 5.5: a) XRD-Spektren von Proben mit steigendem Mn-Angebot (von unten nach oben) und b) steigendem Gesamtmetall-Angebot (von unten nach oben). Der jeweils andere Parameter wurde konstant gehalten, ebenso wie die Wachstumstemperatur, die bei $650^{\circ} \mathrm{C}$ lag (vergleiche auch Abbildung 5.6). Signale vom Al-Probenhalter sind mit „*“ markiert, Signale vom AlN-Puffer mit "+*.

[200]-Richtung orientiert, bei höherem Mn-Anbebot dominiert dann die [111]-Richtung. Abbildung 5.5b) zeigt, was sich bei konstantem relativen Mn-Angebot von $\delta_{M n}=15 \%$ und steigendem Gesamtmetall-Angebot ändert. Im Stickstoffreichen wird das Mn verdünnt in das GaN eingebaut und ab einer bestimmten Schwelle sind sofort starke Signale von Ausscheidungen detektierbar.

Die Ergebnisse dieser Röntgenuntersuchungen lassen sich mit Abbildung 5.6 zusammenfassen, dem so genannten ,spider web“. In dieser Auftragung des Parameter-Raums des MBE-Wachstums von GaMnN werden Ga- und Mn-Fluss gegeneinander aufgetragen, die Punkte markieren die gewachsenen Proben. Der Stickstofffluss wird dabei für alle Proben konstant gehalten. Es wurde ein zusammenhängender Bereich gefunden, in dem reines GaMnN ohne Ausscheidungen hergestellt werden kann. Dieser liegt im sehr stickstoffreichen Bereich bei niedrigem bis moderatem Mn-Angebot. Der höchste verdünnte Mn-Einbau wurde in Probe J0117 mit 5.0\% erreicht. Dies ist genau die Konzentration, von der Dietl et al. [9] ausgingen als sie Ferromagnetismus bei Raumtemperatur vorhersagten. Damit ist es gelungen, GaMnN-Proben herzustellen, die nach den theoretischen Vorhersagen das Potential zum Raumtemperatur-Ferromagneten haben, zumindest was 


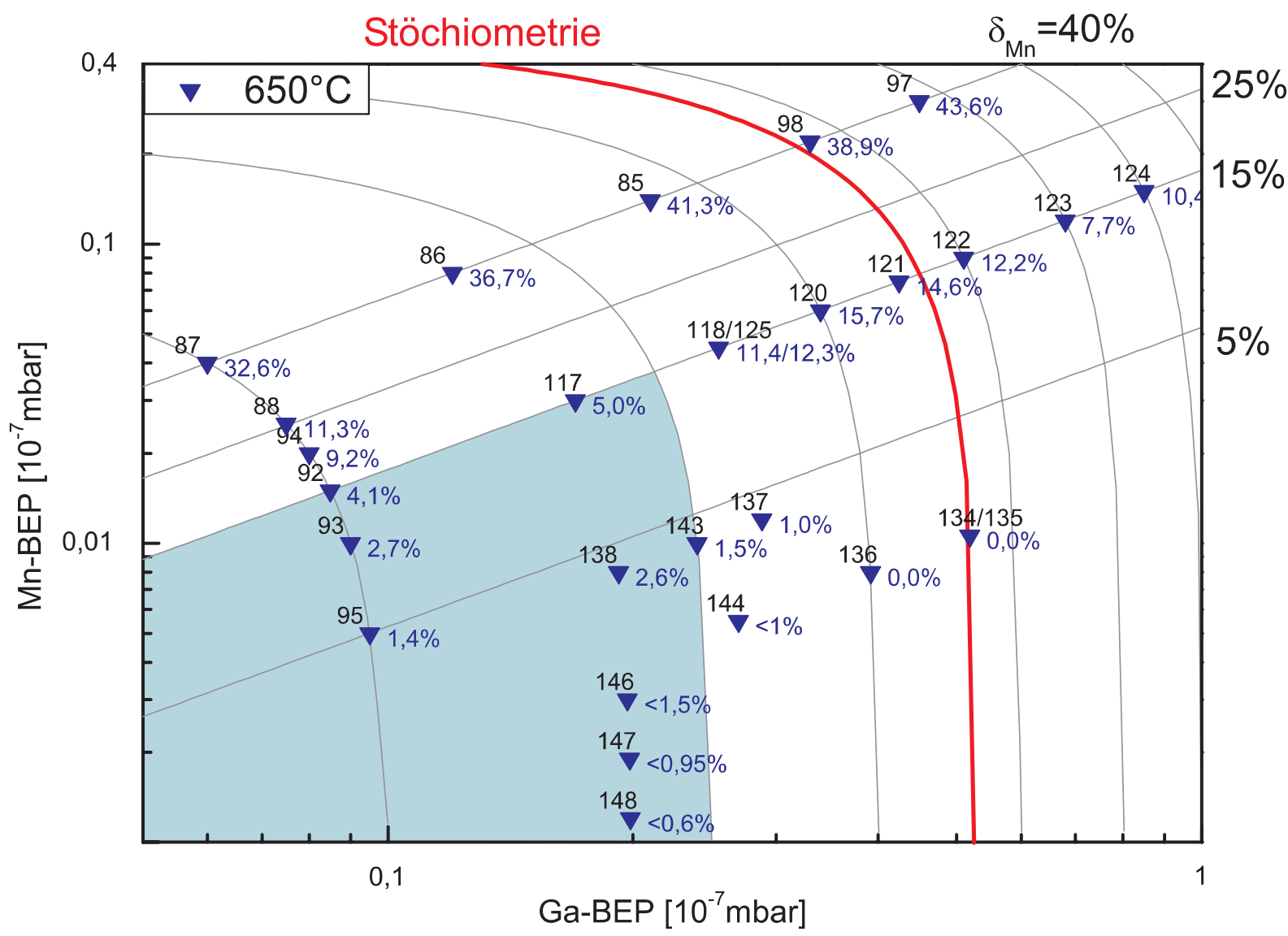

Abbildung 5.6: Wachstumsfenster im Parameter-Raum zum Herstellen von reinem GaMnN, markiert mit hellblau. Die blauen Dreiecke markieren gewachsene Proben mit der jeweiligen Probennummer in schwarz daneben. Die Prozentzahlen geben den Mn-Einbau laut EDX an. Die Proben außerhalb des Wachstumsfensters zeigten Ausscheidungen.

die Mn-Konzentration betrifft.

\section{Zusammenfassung}

In diesem Kapitel wurde gezeigt, dass es beim Wachstum von GaMnN zu Ausscheidungen einer zweiten Phase kommen kann. Diese zweite Phase wurde mittels XRD nachgewiesen und mit Hilfe von XRD- und lokalen EDX-Messungen als $\mathrm{GaMn}_{3} \mathrm{~N}$ identifiziert. Schließlich wurde ein Wachstumsfenster gefunden, in dem GaMnN ohne Ausscheidungen auf $\operatorname{Si}(111)$ gewachsen werden kann und variable Konzentrationen bis 5\% reproduzierbar erreicht werden. Dazu sind niedrige Wachstumstemperaturen und stickstoffreiches Wachstum notwendig. 


\subsection{Strukturelle Eigenschaften von GaMnN}

Die Kristallqualität eines Halbleiters entscheidet darüber, ob die theoretisch erwarteten Eigenschaften auch praktisch erreicht werden können. Denn bei schlechter Kristallqualität, also vielen ausgedehnten Defekten, werden optische und elektrische Eigenschaften des Materials beeinträchtigt. Zur Bestimmung der Kristallqualität gibt es nicht die Messung oder den Parameter, aber durch die weitreichenden Auswirkungen haben sich mehrere Messgrößen als Maß für die Kristallqualität eingebürgert. Beispielsweise werden die Breiten der Röntgensignale (siehe Kapitel 4.3.4) häufig als Standard-Parameter im Schichtwachstum bestimmt, da sie direkt mit strukturellen Eigenschaften korreliert sind. Andererseits liefern auch optische Messungen wie PL oder Raman (Kapitel 4.1 bzw. 4.2) wertvolle Hinweise auf die elektronische bzw. strukturelle Qualität.

\subsubsection{Veränderung mit der Wachstumstemperatur}

Optimal für das MBE-Wachstum von GaN sind Wachstumstemperaturen von $750^{\circ} \mathrm{C}$ bis $800^{\circ} \mathrm{C}$, je nach Detailausführung der Anlage. Für das Wachstum von GaMnN auf $\operatorname{Si}(111)$ wurden Temperaturen von $760^{\circ} \mathrm{C}$ (GaN-optimiert), $730^{\circ} \mathrm{C}$ und $650^{\circ} \mathrm{C}$ benutzt, wobei der Einbau nur bei tiefen Temperaturen kontrollierbar war [66]. Man erwartet allerdings bei diesen Temperaturen eine schlechtere Kristallqualität.

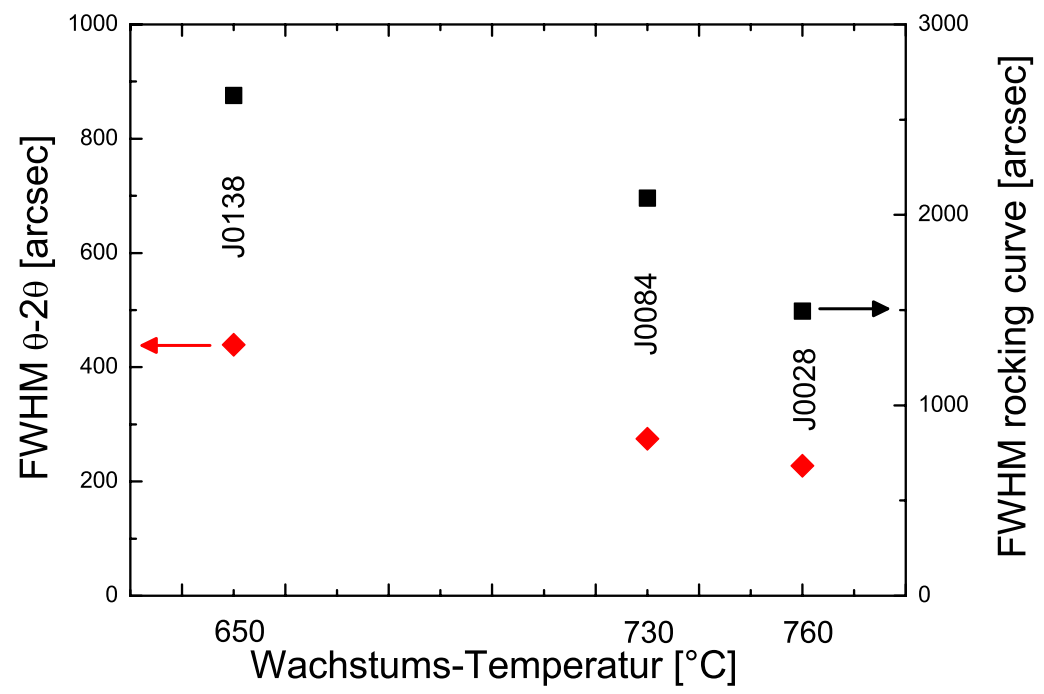

Abbildung 5.7: FWHM der Röntgenmessungen am (0002)-Reflex von GaMnN bei unterschiedlicher Wachstumstemperatur. Der Messfehler liegt unterhalb der Symbolgröße. Die Kristallqualität nimmt mit sinkender Wachstumstemperatur ab.

Genau dieses Verhalten wird auch experimentell beobachtet, wie Abbildung 5.7 zeigt. Mit sinkender Wachstums-Temperatur nimmt sowohl die Breite der $\theta$-2 $\theta$-Signale als auch die der rocking curve des (0002)-Signals von GaMnN zu. Die Mn-Konzentration der Proben J0028 und J0084 liegt bei etwa 0.3\%, auf Grund des erwähnten schlechten Einbaus bei hohen Temperaturen. Probe J0138 enthält 2.6\% Mn. Der Trend zu schlechterer Kris- 
tallqualität ist dabei nicht auf den höheren Mn-Gehalt bei niedrigeren Temperaturen zurückführbar, da er unabhängig vom Mn auch in GaN-Referenzproben beobachtbar ist.

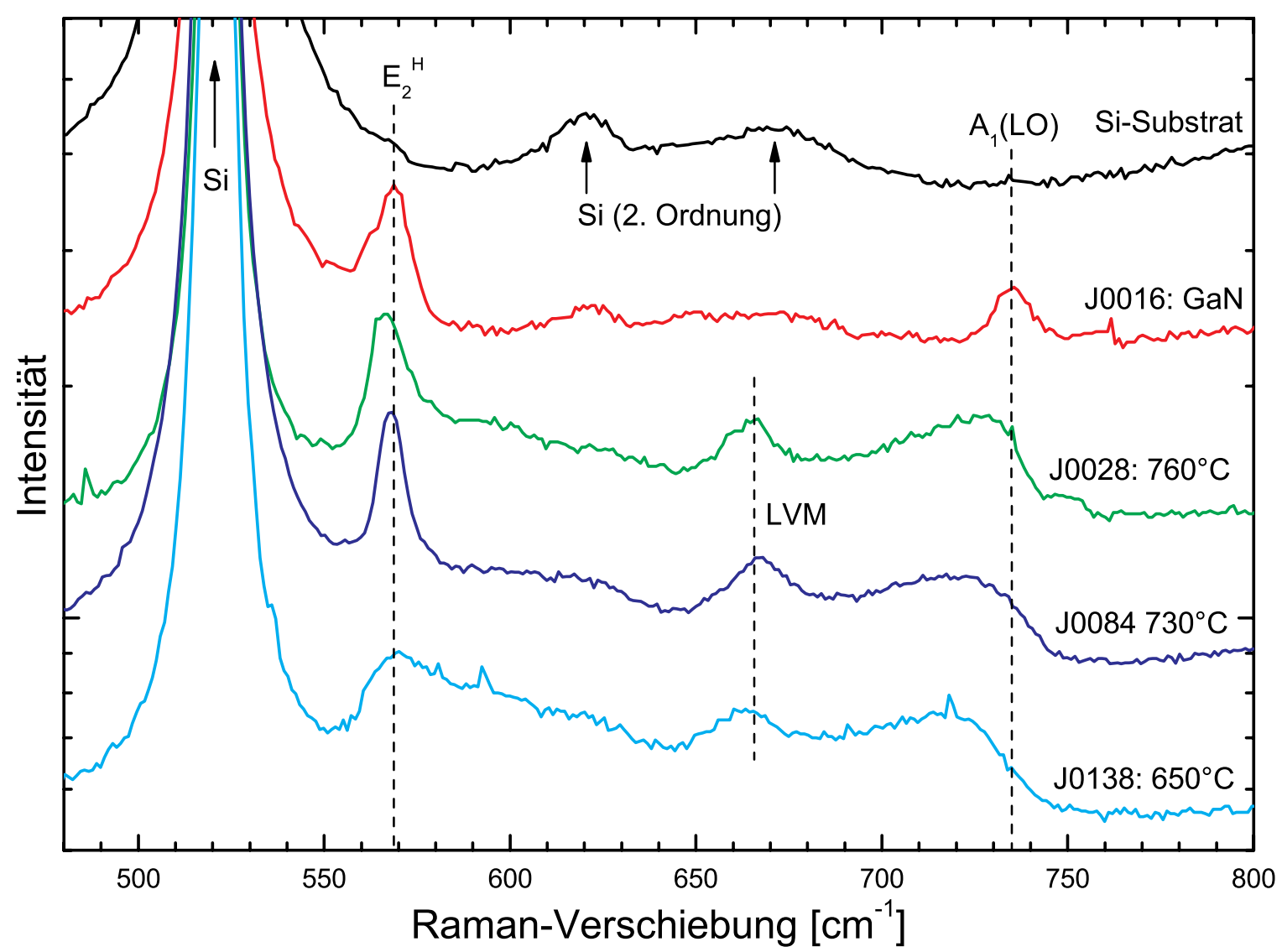

Abbildung 5.8: Raman-Spektren von GaMnN in Abhängigkeit von der Wachstumstemperatur. Zum Vergleich ein Spektrum des Si-Substrates und einer GaN-Referenzprobe, gewachsen bei $760^{\circ} \mathrm{C}$. Die Literaturwerte für Volumenproben sind für die beiden GaN-Moden markiert.

Abbildung 5.8 zeigt Raman-Messungen an denselben Proben, sowie zwei Vergleichsmessungen am Si-Substrat und J0016, einer GaN-Referenzprobe zu J0028 bei $760^{\circ} \mathrm{C}$. Die Spektren werden dominiert vom optischen Phonon von Si, welches bei $521 \mathrm{~cm}^{-1}$ zu sehen ist. Da die drei optischen Moden von Si am $\Gamma$-Punkt entartet sind, ist in erster Ordnung kein weiteres Signal zu erwarten. Allerdings ist das Si-Signal so stark, dass auch RamanEffekte zweiter Ordnung (siehe Kapitel 4.2) sichtbar werden (siehe Abbildung). In der GaN-Referenzprobe J0016 sind diese Signale ebenfalls noch schwach erkennbar, zusätzlich zu den $E_{2^{-}}$und $A_{1}(L O)$-Moden, die in dieser Geometrie zu erwarten sind. Die $E_{2}^{H}$-Mode zeigt keine signifikante Abweichung vom Volumenwert von $569 \mathrm{~cm}^{-1}$ [82], woraus folgt, dass die Probe unverspannt ist. Die normalerweise bei Si-Substraten auftretenden tensilen Verspannungen sind in dieser Probe nicht nachweisbar. Auch die $A_{1}(L O)$-Mode zeigt keine Verschiebung, was auf eine geringe Ladungsträgerkonzentration hindeutet. Bei Konzentrationen oberhalb $\approx 10^{17} \mathrm{~cm}^{-3}$ würde diese Linie durch Phonon-Plasmon-Kopplung 
zu höheren Energien verschoben werden.

Die GaMnN-Proben zeigen einen wesentlichen Unterschied an der $A_{1}(L O)$-Mode. Anstelle einer Linie bei $735 \mathrm{~cm}^{-1}$ ist hier ein breites, asymmetrisches Signal zwischen 700 und $740 \mathrm{~cm}^{-1}$ erkennbar. Dieses Signal lässt sich auf disorder-activated Raman scattering (DARS) zurückführen. Wie in Kapitel 4.2 beschrieben ist DARS ein Zeichen dafür, dass die kristalline Ordnung auf größeren Längenskalen gestört ist, weshalb die RamanAuswahlregeln gelockert werden. Dies deutet auf eine wesentlich schlechtere Kristallqualität durch Mn-Einbau hin. Da DARS an der $A_{1}(L O)$-Mode bei allen WachstumsTemperaturen ab einer Mn-Konzentration von etwa $0.5 \%$ beobachtbar war, ist er nicht Temperatur-spezifisch. Ein weiterer Temperatur-unabhängiger Effekt ist das Auftreten einer lokalen Mode (LVM) bei $666 \mathrm{~cm}^{-1}$, die auch schon von Harima [114] in GaMnN beschrieben wurde. Diese Mode wird von Limmer et al. [115] einer Vakanz im GaN-Gitter zugeordnet ohne genauere Spezifizierung. Auf Grund der stickstoffreichen Wachstumsbedingungen ist in den hier vorgestellten Proben die Bildung von Ga-Vakanzen wahrscheinlich, andererseits können als kompensierender Defekt zum tiefen Mn-Akzeptor N-Vakanzen eingebaut werden, wie von Kane et al. angenommen wird [116].

Generell sind die Spektren von J0028 und J0084 sehr ähnlich. Nur Probe J0138 zeigt eine signifikante Abweichung an der $E_{2}^{H}$-Mode. Anstelle einer scharfen Linie ist diese Mode extrem verbreitert und zeigt auf der hochenergetischen Flanke keine scharfe Begrenzung mehr zum Untergrund. Dies kann zwei verschiedene Ursachen haben: Einerseits könnte es sich um DARS handeln, da die Phononen-Zustandsdichte in diesem Spektralbereich qualitativ diese verbreiterte Form aufweist [114] (siehe auch Abbildung 4.7). Andererseits wurden genau im Bereich $580 \mathrm{~cm}^{-1}$ bis $590 \mathrm{~cm}^{-1}$ von einigen Gruppen lokale Mn-N-Moden identifiziert [117, 114, 118], wie es auch nach Gleichung 4.19 erwartet wird. Allerdings sollte das Auftreten von lokalen Moden nicht die ursprüngliche scharfe $E_{2}^{H}$-Mode verändern, so dass für J0138 zumindest auch von einem Unordnungs-Effekt ausgegangen werden muss. Dies zeigt eine noch einmal wesentlich verschlechterte Kristallqualität im Vergleich zu den Proben bei $760^{\circ} \mathrm{C}$ und $730^{\circ} \mathrm{C}$. Während also XRD eine kontinuierliche Verschlechterung der Qualität mit sinkender Wachstums-Temperatur zeigt, ist durch Raman-Messungen kein Unterschied zwischen $760^{\circ} \mathrm{C}$ und $730^{\circ} \mathrm{C}$ sichtbar, dafür aber ein qualitativer Sprung $\mathrm{zu} 650^{\circ} \mathrm{C}$, der sich im Auftauchen einer weiteren DARS-Mode äußert. Allerdings muss bei den Raman-Messungen auch berücksichtigt werden, dass die Mn-Konzentration in Probe J00138 $\left(650^{\circ} \mathrm{C}\right)$ wesentlich höher ist, weshalb nun der Einfluss der Mn-Konzentration untersucht werden soll.

\subsubsection{Einfluss von Ga- und Mn-Angebot}

Auf Grund des bereits angesprochenen Zwanges, niedrige Wachstumstemperaturen zu wählen um Mn einzubauen, wurden sehr viele Proben bei $650^{\circ} \mathrm{C}$ gewachsen. Mit dieser großen Probenauswahl sollte sichergestellt werden, dass aus diesen eigentlich schlechten Bedingungen das Optimum bei den anderen Wachstums-Parametern erreicht wird. Diese Parameter sind das Verhältnis von Metall- zu Stickstoff-Fluss, sowie der relative MnAnteil, wie schon bei dem ,spider web“ in Kapitel 5.1 .3 gezeigt.

Alle Proben im Bereich des Wachstums ohne Ausscheidungen zeigen vergleichbare Wer- 


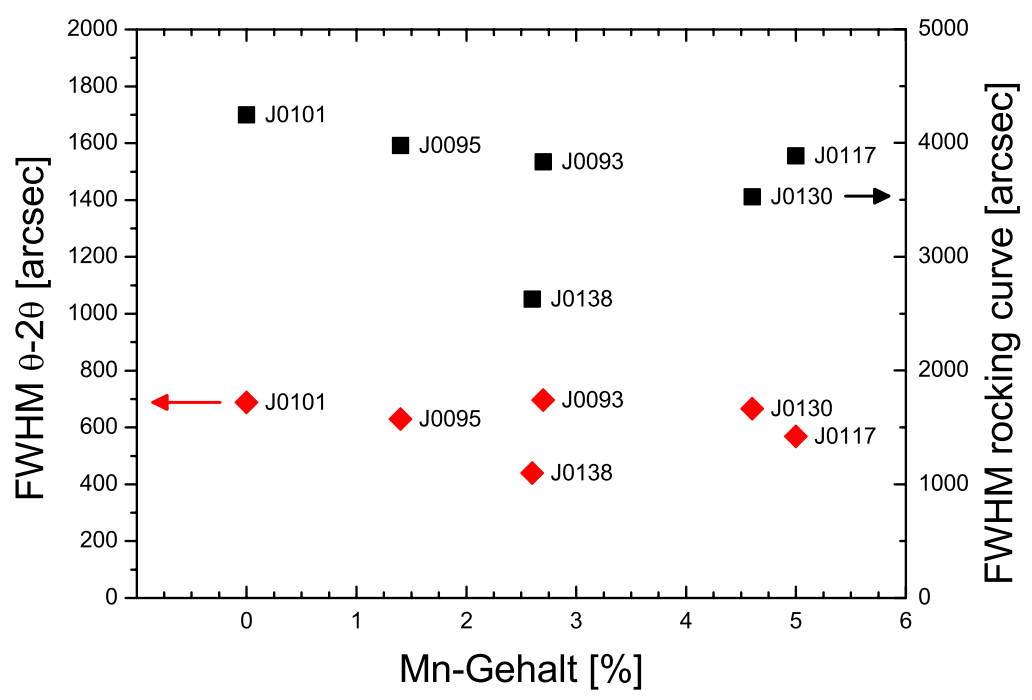

Abbildung 5.9: FWHM der Röntgenmessungen am (0002)-Reflex von GaMnN ohne Ausscheidungen mit unterschiedlichem Mn-Gehalt. Der Messfehler liegt unterhalb der Symbolgröße. Die Kristallqualität zeigt keine signifikante Abhängigkeit von der Mn-Konzentration.

te bei den Breiten der Röntgensignale. Die Abweichungen voneinander lassen sich durch unkontrollierbare Einflüsse während des Wachstums erklären, wie etwa kleine Temperaturschwankungen oder wechselnde Hintergrunddrücke. Das bedeutet damit auch, dass im Rahmen dieser Schwankungen verdünnte GaMnN-Proben mit gleichbleibender Qualität gewachsen werden können, mit beliebig einstellbarem Mn-Gehalt von 0 bis $5 \%$.

Erweitert man den Parameterraum um die Proben mit Ausscheidungen, so kann man zweierlei beobachten. Wird erstens bei konstantem Gesamtmetall-Fluss der Mn-Anteil erhöht (vergleiche Abbildung 5.5a)), so verschlechtert sich die Kristallqualität von GaN mit steigendem Anteil an Ausscheidungen. Zweitens wird bei konstantem relativen MnAngebot und Änderung des Wachstums von stickstoff- zu metallreich (vergleiche Abbildung 5.5b)) die Kristallqualität trotz Ausscheidungen besser. Dieser Effekt kann darauf zurückgeführt werden, dass generell unter stickstoffreichen Bedingungen eine schlechtere Qualität erreicht wird als unter leicht metallreichen Bedingungen $[64,58]$.

Abbildung 5.10 zeigt Raman-Messungen bei variierendem Ga-Fluss. Die genauen Wachstumsparameter der Proben sind aus dem „spider web“ (Abbildung 5.6) ersichtlich. Hier ist ein ähnlicher Effekt beobachtbar wie bei den XRD-Messungen. Die Spektren von J0135 und J0136, die nahe der Stöchiometrie gewachsen sind und daher trotz MnKonzentrationen unterhalb der EDX-Nachweisgrenze Ausscheidungen zeigen, sind vergleichbar mit J0108, einer GaN-Referenzprobe nahe der Stöchiometrie. Bei Probe J0136 ist die $E_{2}^{H}$-Linie allerdings schon sichtbar verbreitert, was bereits auf eine leichte Verschlechterung der Qualität hindeutet. Bei Probe J0137 mit 1\% Mn und Ausscheidungen tritt eine weitere Verbreiterung auf und die $A_{1}$-Mode wird durch DARS ersetzt, ein weiteres starkes Zeichen für abnehmende Kristallqualität. Bei der verdünnten Probe J0138 ohne Ausscheidung schließlich ist auch die $E_{2}^{H}$-Mode durch DARS ersetzt, wie bereits in Kapitel 5.2.1 analysiert. Die Proben bei noch geringerem Metall-Fluss sind auf Grund der 


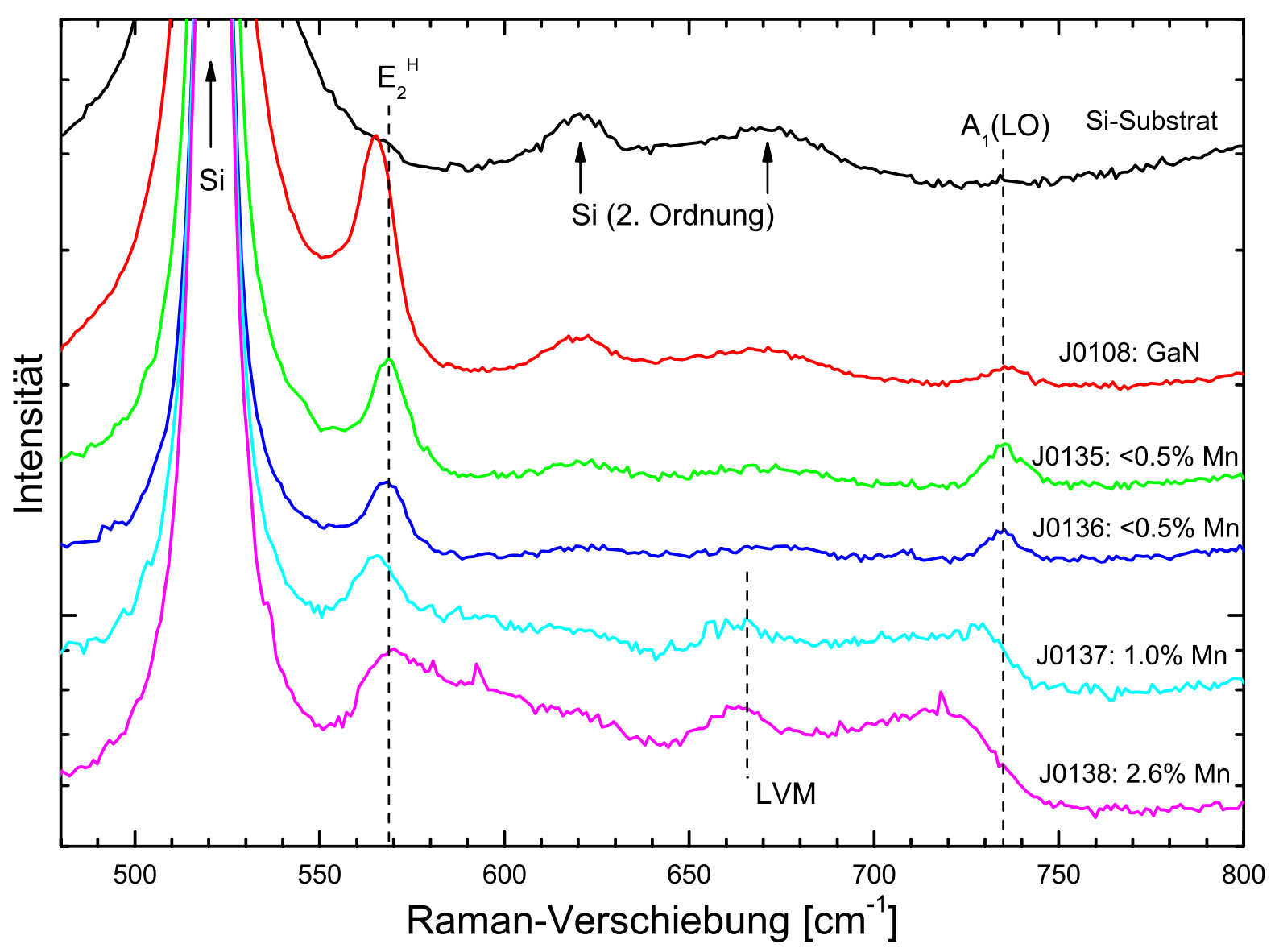

Abbildung 5.10: Raman-Spektren von GaMnN in Abhängigkeit von den Wachstumsbedingungen. Bei ungefähr konstantem Mn-Fluss wird der Ga-Fluss von J0135 bis J0138 erniedrigt, nur J0138 zeigt keine Ausscheidungen.

langsamen Wachstumsrate zu dünn, um verwertbare Raman-Signale zu erhalten, so dass hier Vergleichsmessungen fehlen.

Die LVM bei $666 \mathrm{~cm}^{-1}$ kann hier wiederum nicht klar einer der Vakanzen $\mathrm{V}_{G a}$ oder $\mathrm{V}_{N}$ zugeordnet werden. Es zeigt sich ein klares Signal in den stickstoffreich gewachsenen Proben J0137 und noch stärker in J0138, obwohl die Schichtdicke und damit Gesamtintensität mit zunehmendem Stickstoffreichtum abnimmt. Nahe der Stöchiometrie fehlt das Signal völlig. Dies deutet auf $\mathrm{V}_{G a}$ als zu Grunde liegenden Defekt hin. Diese Zuordnung ist auch in Übereinstimmung mit theoretischen Rechnungen [119, 120], die unter stickstoffreichen Bedingungen niedrigere Bildungsenergie als unter galliumreichen Bedingungen für $\mathrm{V}_{G a}$ vorhersagen. Allerdings sind die stickstoffreicheren Proben gleichzeitig diejenigen, die eine höhere Mn-Konzentration aufweisen. Die gleichen theoretischen Berechnungen [119, 120] liefern bei einem Ferminiveau tief in der Bandlücke, wie es durch Mn erzwungen wird, eine Bevorzugung von $\mathrm{V}_{N}$. 


\subsubsection{Einfluss des Substrates}

Die umfangreichen Optimierungen von GaMnN wurden alle auf Si(111)-Substrat mit einem AlN-Puffer durchgeführt. Dieses Substrat ist zwar leicht erhältlich und billig, allerdings zeigen GaN-Schichten die darauf gewachsen werden immer eine vergleichsweise schlechte Qualität, obwohl beispielsweise die eigentlich zu erwartende tensile Verspannung in den untersuchten Schichten nur schwach ausgeprägt war (siehe Abbildung 5.8 und 5.10). Im Vergleich dazu ist GaN auf Saphir immer kompressiv verspannt, erreicht aber typischerweise etwas höhere Kristallqualität. Außerdem wird durch die hier verwendete Pufferschicht aus MOCVD-gewachsenem GaN eine viel niedrigere Versetzungsdichte erreicht, da MOCVD-Wachstum bei deutlich höheren Temperaturen $\left(1125^{\circ} \mathrm{C}\right)$ stattfindet. Allerdings werden die meisten Analysen beim Wachstum auf GaN-Puffern dadurch erschwert, dass der Anteil der Pufferschicht beispielsweise am Röntgen- oder Ramansignal mit berücksichtigt werden muss.

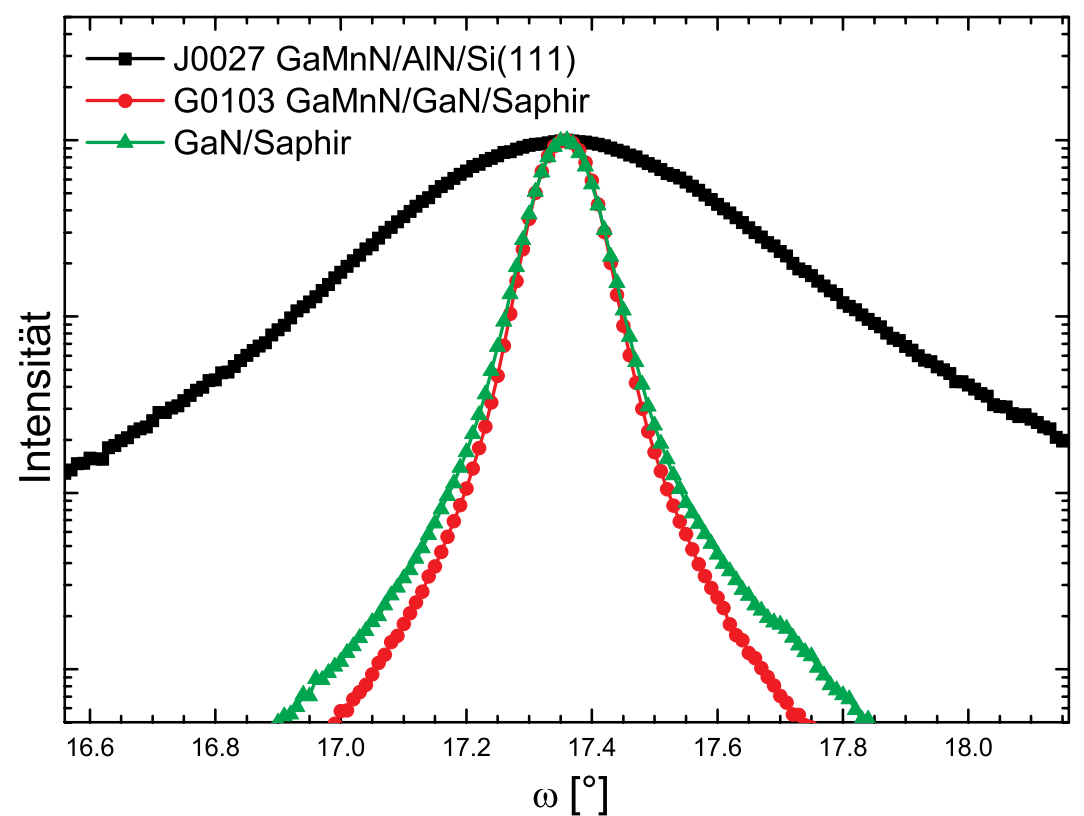

Abbildung 5.11: Vergleich der rocking curves am (0002)-Reflex von Proben auf unterschiedlichen Substraten. Zum Vergleich ist die Kurve der reinen Pufferschicht auf Saphir abgebildet. Alle Spektren wurden normiert und auf der $\mathrm{x}$-Achse verschoben, so dass die Maxima übereinander liegen.

In Abbildung 5.11 sind zwei GaMnN-Proben gezeigt, die bei hohen Temperaturen auf unterschiedlichen Substraten gewachsen wurden. Probe G0103 (auf Saphir) zeigt eine fast identische Kurve wie der GaN-Puffer, auf dem sie gewachsen wurde. Nur im Randbereich kann man erkennen, dass die Kristallqualität der GaMnN-Schicht tatsächlich noch etwas höher ist als die des MOCVD-Puffers. Passt man an den GaN-Puffer eine Gauss-Funktion an und benutzt deren Breite als fixen Parameter für die Anpassung von zwei Gauss-Kurven an Probe G0103, so ergeben sich die in Tabelle 5.3 angegebenen Halbwertsbreiten. Das 
Intensitätsverhältnis der beiden Gauss-Kurven wurde dabei auf dem Verhältnis der Dicken der beiden Schichten festgehalten.

\begin{tabular}{|c|c|}
\hline Probe (Substrat) & FWHM rocking curve \\
\hline J0027 (Si) & $1628 \pm 7$ \\
G0103 (Saphir) & $274 \pm 21$ \\
GaN-Puffer (Saphir) & $303 \pm 1$ \\
\hline
\end{tabular}

Tabelle 5.3: Halbwertsbreiten der rocking curves aus Abbildung 5.11.

Auch diese numerische Anpassung zeigt die leicht erhöhte Kristallqualität der Schicht G0103 gegenüber dem MOCVD-Puffer. Im Vergleich zu Probe J0027 auf Si(111) konnte die Halbwertsbreite um den Faktor 6 gesenkt werden, was eine entscheidende Verbesserung bedeutet. Der vergleichsweise große Fehler bei G0103 ergibt sich aus der Anpassung von zwei verschiedenen Gauß-Funktionen und zeigt die erhöhte Unsicherheit in der Auswertung von Proben, die auf einem dicken GaN-Puffer gewachsen wurden.

Raman-Messungen an Proben auf Saphir-Substrat mit GaN-Puffer zeigen qualitativ das gleiche Verhalten wie die Proben auf Si(111). Allerdings lässt sich hier auf Grund des viel schlechteren Signal-Rausch-Verhältnisses im Vergleich zu XRD keine quantitative Aussage ableiten. Es kann lediglich festgestellt werden, dass bei Einbau im ProzentBereich DARS auftritt, der aber noch überlagert ist durch die ungestörten GaN-Moden des Puffers. Proben mit niedrigem Mn-Gehalt sind qualitativ nicht zu unterscheiden von Referenzproben ohne Mn.

\section{Zusammenfassung}

In diesem Kapitel wurde gezeigt, dass die Kristallqualität der GaMnN-Proben wie zu erwarten mit niedrigen Wachstumstemperaturen sinkt. Dies äußert sich in verbreiterten Röntgen-Signalen und DARS-Signalen in Raman-Spektren. Die Proben aus dem identifizierten Wachstumsfenster von GaMnN zeigen eine relativ konstante Kristallqualität. Die Qualität kann durch den Wechsel des Substrats auf Saphir (mit einem MOCVDgewachsenen GaN-Puffer) erheblich erhöht werden. 


\subsection{RBS-Messungen und Gitterplatzbestimmung von $\mathrm{Mn}$}

RBS liefert Informationen über Schichtzusammensetzungen und -dicken der gemessenen Proben. Es wurde daher als unabhängige Bestätigung der EDX-Messungen eingesetzt um die Mn-Konzentration zu bestimmen. Außerdem kann die Schichtdicke exakter als mit einem Oberflächenprofilometer (Dektak) bestimmt werden. Abbildung 5.12 zeigt die Ergebnisse von Probe J0117, eine GaMnN-Probe ohne Ausscheidungen mit einem MnGehalt von 5\% laut EDX. Eine Anpassung und Simulation des RBS-Spektrums mittels
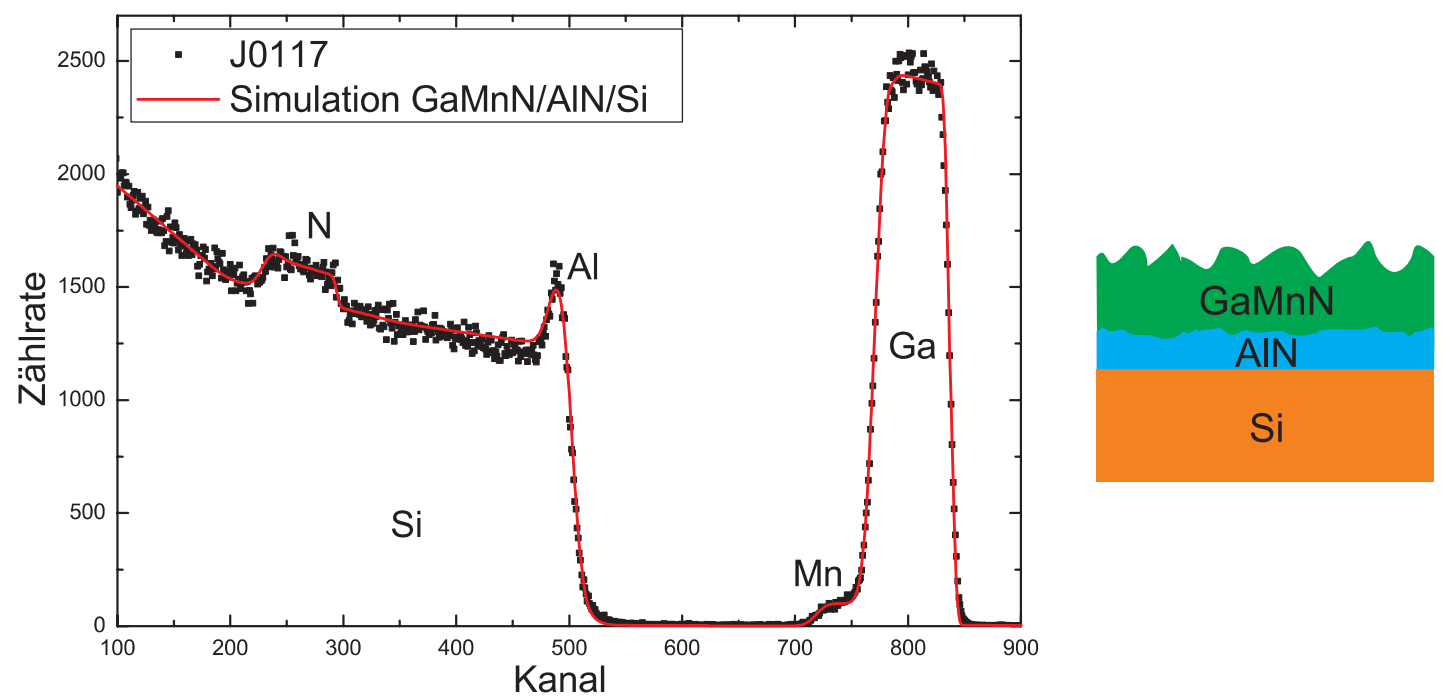

Abbildung 5.12: RBS-Spektrum von Probe J0117 mit Simulation (links). Rechts schematisch die Schichtfolge mit rauen Grenzflächen.

des Softwarepaketes RUMP [95] ist ebenfalls gezeigt. Mit den in Tabelle 5.4 genannten Parametern ergibt sich eine sehr gute Übereinstimmung zwischen Messung Simulation. Die Abweichung der Mn-Konzentration zwischen EDX- und RBS-Messung liegt innerhalb normaler Fehler beider Methoden. Diese liegen im Bereich von $0.5-1 \%$.

Eine Fehlerquelle der RBS-Anpassung liegt dabei in der Behandlung der Rauigkeit der Schichten. Diese wird dadurch simuliert, dass eine normalverteilte Schichtdicke über die laterale Ausdehnung der Probe angenommen wird, deren Mittelwert als „Schichtdicke“ und deren Standardabweichung als „Rauigkeit" bezeichnet wird. Gerade bei kolumnarem Wachstum können sich aber charakteristische Dickenverteilungen durch die Facettierung der Seitenflächen ergeben, die nicht zwangsläufig einem (statistischen) Gauss-Profil entsprechen. Dies erkennt man beispielsweise am Übergang zwischen Ga- und Mn-Signal, wo Simulation und Messung nicht perfekt übereinstimmen. Durch diesen Fehler wird der MnGehalt bei der RBS-Messung tendenziell überschätzt, wie auch der Vergleich mit EDX zeigt. Proben mit kleinerem Mn-Gehalt wie J0091 sind für diesen Fehler noch deutlich anfälliger, so dass auf weitere RBS-Messungen zur Konzentrationsbestimmung verzichtet wurde und EDX als Standardmethode verwendet wurde.

Die Schichtdickenanalyse mit RBS ist sehr präzise, weil das Ga-Signal sehr scharfe 


\begin{tabular}{|l||c|c|c|}
\hline & J0091 & J0093 & J0117 \\
\hline Mn-Konzentration (RBS) [\%] & $7.0 \pm 0.2$ & $2.9 \pm 0.1$ & $6.2 \pm 0.1$ \\
Mn-Konzentration (EDX) [\%] & $2.6 \pm 0.2$ & $2.7 \pm 0.2$ & $5.0 \pm 0.4$ \\
Schichtdicke GaMnN (RBS) [nm] & $58.2 \pm 0.5$ & $67.3 \pm 0.4$ & $132.9 \pm 0.3$ \\
Rauigkeit GaMnN (RBS) [nm] & $32.9 \pm 0.4$ & $28.2 \pm 0.3$ & $19.9 \pm 0.3$ \\
Schichtdicke GaMnN (Dektak) $[\mathrm{nm}]$ & $103 \pm 46$ & $135 \pm 48$ & $153 \pm 56$ \\
\hline
\end{tabular}

Tabelle 5.4: Ergebnisse der RBS-Messungen an drei verdünnten GaMnN-Proben im Vergleich mit anderen Charakterisierungen durch EDX und Dektak.

Flanken aufweist. Da die von RBS bestimmte Dicke letztendlich der Atomflächendichte entspricht, kann hiermit die Wachstumsrate auch deutlich genauer bestimmt werden als mit Oberflächenprofilometrie. In der Profilometrie wird eine Nadel über die Oberfläche geführt, die einen mit der Oberflächenrauigkeit verglichen großen Spitzenradius aufweist. Als Folge misst man die Einhüllende des Oberflächenprofils, was zu einer systematischen Überschätzung der Schichtdicke und damit der Wachstumsrate führt. Dieses Problem tritt besonders bei den Proben mit niedrigem Metallfluss auf, da im extrem stickstoffreichen Bereich die Oberflächenrauigkeit hoch ist. Dies wurde im Rahmen der Diplomarbeit von Du Mai [57] auch mit Rasterkraftmikroskopie (AFM) untersucht. Dort ergaben sich Profile, bei denen der Abstand von Minimum und Maximum der Messung ungefähr der Schichtdicke laut Dektak entsprach. Das bedeutet, dass es tatsächlich „Löcher" in der Schicht gibt, die bis zum AlN-Puffer reichen.

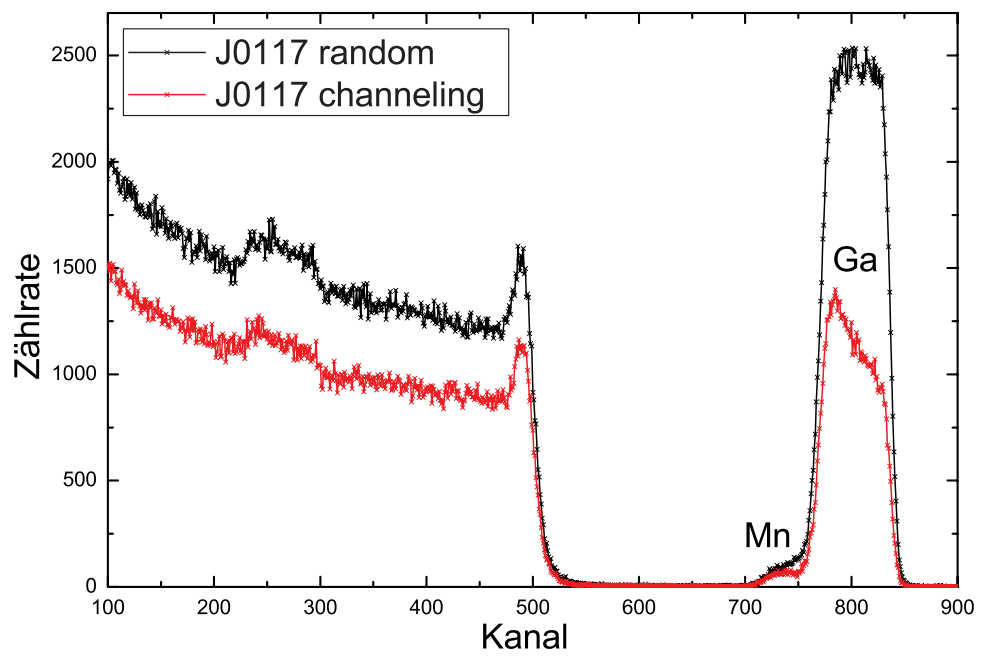

Abbildung 5.13: RBS-Spektrum von Probe J0117 in channeling-Geometrie im Vergleich zur randomMessung. Der channeling-Effekt ist nur schwach ausgeprägt und wird mit zunehmender Tiefe schlechter.

Zusätzlich zu den normalen RBS-Messungen in „,random“-Geometrie wurden RBS/CMessungen in „,channeling“-Geometrie durchgeführt (siehe Kapitel 4.3.2). Abbildung 5.13 
zeigt das Ergebnis für Probe J0117. Man erkennt den Channeling-Effekt an der reduzierten Zählrate. Allerdings sinkt die Zählrate nach dem Oberflächensignal nicht stark ab, wie es bei hoher Kristallqualität und damit gutem channeling der Fall wäre, sondern sie steigt weiter kontinuierlich an. Die $\mathrm{He}^{2+}$-Teilchen können sich also nicht frei durch die Kanäle in der Wurtzit-Struktur bewegen, sondern werden durch Defekte aus ihrer ursprünglichen Richtung gestreut und tragen dann zu einem RBS-Signal vergleichbar der random-Messung bei. Dies ist in Übereinstimmung mit den anderen Messungen zur Kristallqualität und TEM-Untersuchungen, die viele ausgedehnte Defekte zeigen [113].

Ziel der RBS/C-Messungen war es, eine Information über den Gitterplatz von Mn in GaMnN zu erhalten. Dazu würde die normalerweise konstante Zählrate hinter dem Oberflächensignal im Verhältnis zur random-Zählrate (der so genannte „channeling yield“") von Mn und Ga vergleichen und dann winkelabhängig messen. Wie man allerdings in Abbildung 5.13 erkennt ist diese konstante Zählrate nicht vorhanden. Damit ist eine seriöse Auswertung nicht möglich. Bestenfalls erkennt man, dass der channeling yield für Mn höher als für Ga ist. Dies könnte darauf hindeuten, dass Mn auf interstitiellen Plätzen eingebaut wird. Eine andere mögliche Erklärung liegt aber in der Überlagerung des Mn-Signals mit Ausläufern des Ga-Signals, die zu einer Überschätzung des Mn-Gehalts geführt haben. Diese Ausläufer des Ga-Signals entsprechen genau den am tiefsten liegenden Ga-Atomen, die damit einen wesentlich schlechteren channeling yield aufweisen als die (nahe der Oberfläche liegenden) Mn-Atome bei gleicher Rückstreuenergie. Auf Grund dieser Erkenntnis ist für die Gitterplatz-Bestimmung eine lokale Methode vonnöten, da die Kristallqualität auf makroskopischer Skala nicht ausreichend ist. Von Tore Niermann wurde im Rahmen seiner Doktorarbeit dazu die ALCHEMI(atom localisation by channeling enhanced microanalysis)-Technik am TEM weiterentwickelt und auf die vorliegenden Proben angewandt [113, 121]. Dabei ergab sich für Mn zu 96.5 $\pm 5.0 \%$ ein Einbau auf substitutionellen Ga-Plätzen.

Auch andere Gruppen konnten den Gitterplatz von Mn als größtenteils substitutionell bestimmen [122, 123, 124, 125]. Meist wurde dabei auf EXAFS als Methode zurückgegriffen [122, 123, 124], in einem Fall auch auf in-situ direct recoil spectroscopy (DRS) [125]. In beiden Fällen wird dabei über einen größeren Bereich der Probe gemittelt, wobei EXAFS auf das Volumen und DRS auf die Oberfläche sensitiv ist. Außerdem ist jeweils keine genaue Quantifizierung möglich, sondern nur relativ ungenaue Angaben durch Vergleich mit Simulationen ohne echte Anpassung. Die Methode der ALCHEMI hat hier wesentliche Vorteile.

\section{Zusammenfassung}

In diesem Kapitel wurde gezeigt, dass RBS-Messungen für die relativ rauen Proben keine zuverlässige Elementanalyse liefern. Allerdings können die Schichtdickenbestimmungen wesentlich verbessert werden im Vergleich zum Einsatz eines Oberflächenprofilometers. Zur Gitterplatzanalyse mittels RBS/C sind die auf Si-Substrat gewachsenen GaMnNProben auf Grund der beschränkten Kristallqualität nicht geeignet, allerdings konnte mit einer lokalen Methode (ALCHEMI) nachgewiesen werden, dass Mn nahezu vollständig substitutionell auf dem Ga-Gitterplatz eingebaut wird. 


\section{Optische Eigenschaften von GaMnN}

Das Hauptziel dieser Arbeit war das Verständnis der optischen Eigenschaften von GaMnN. Die Unterschiede in den PL-Spektren zu reinem GaN müssen identifiziert und erklärt werden. Dabei ist es wichtig zwischen Effekten zu unterscheiden, die nur aus den geänderten Wachstumsbedingungen des magnetisch dotierten Materials herrühren und solchen, die direkt auf die Anwesenheit von Mn in der GaN-Matrix zurückzuführen sind.

\subsection{PL vom UV bis sichtbaren Bereich}

Bereits in Kapitel 4.1.3 wurden wesentliche Charakteristika der Lumineszenz von GaN angesprochen. Als Vorbereitung zur Analyse von GaMnN sind in Abbildung 6.1 die PLSpektren von zwei Referenzproben abgebildet: Zum einen freistehendes GaN der Firma Lumilog und zum anderen GaN, das mittels MOCVD auf Saphir-Substrat gewachsen wurde. Letzteres wurde auch als Basis für GaMnN-Schichten benutzt.

Anhand dieser Referenz-Spektren lassen sich mehrere Eckwerte festlegen. So kann man in beiden Proben die typische gelbe Lumineszenz bei $2.2 \mathrm{eV}$ beobachten, die im Zusammenhang mit Ga-Leerstellen steht (siehe [73] und dortige Referenzen), so wie bei etwa $3.28 \mathrm{eV}$ einen DAP-Übergang, der auf Verunreinigungen sowohl mit flachen Donatoren als auch flachen Akzeptoren hindeutet. Das freistehende GaN kann als unverspannt betrachtet werden, so dass an Hand dieser Probe der Einfluss von Verspannungen quantifiziert werden kann. Betroffen davon sind insbesondere die bandkantennahen Linien, die nun ins Verhältnis mit veröffentlichter Literatur gebracht werden können. Im vorliegenden freistehenden GaN ist nahe der Bandkante nur ein einziges Signal klar auflösbar, das Donator-gebundene Exziton bei $3.4718 \pm 0.0001 \mathrm{eV}$. Andere Gruppen detektieren diese Linie in unverspanntem GaN im Bereich von 3.471 - 3.472 eV [126, 127, 128, 129].

Im MOCVD-gewachsenen GaN sind nahe der Bandkante mehrere Signale auflösbar. Auf Grund der biaxial kompressiven Verspannung befindet sich die Rekombination des DBX hier bei $3.4849 \pm 0.0001 \mathrm{eV}$. Bei etwa $5.8 \mathrm{meV}$ geringerer Energie ist ein Akzeptorgebundenes Exziton (ABX) sichtbar. Dessen hohe Intensität korreliert mit der im Vergleich zur exzitonischen Lumineszenz hohen Intensität des DAP-Bandes. An der hochenergetischen Flanke der bandkantennahen Lumineszenz sind zwei weitere Signale auflösbar. Das Signal bei $3.4907 \pm 0.0001 \mathrm{eV}$ wird dem freien Exziton A zugeordnet, während bei $3.4994 \pm 0.0010 \mathrm{eV}$ das FXB sichtbar ist.

Die Existenz von freien Exzitonen in PL-Spektren wird allgemein als Hinweis auf eine hohe Materialqualität angesehen. Nur bei niedrigen Defektdichten können freie Exzitonen 


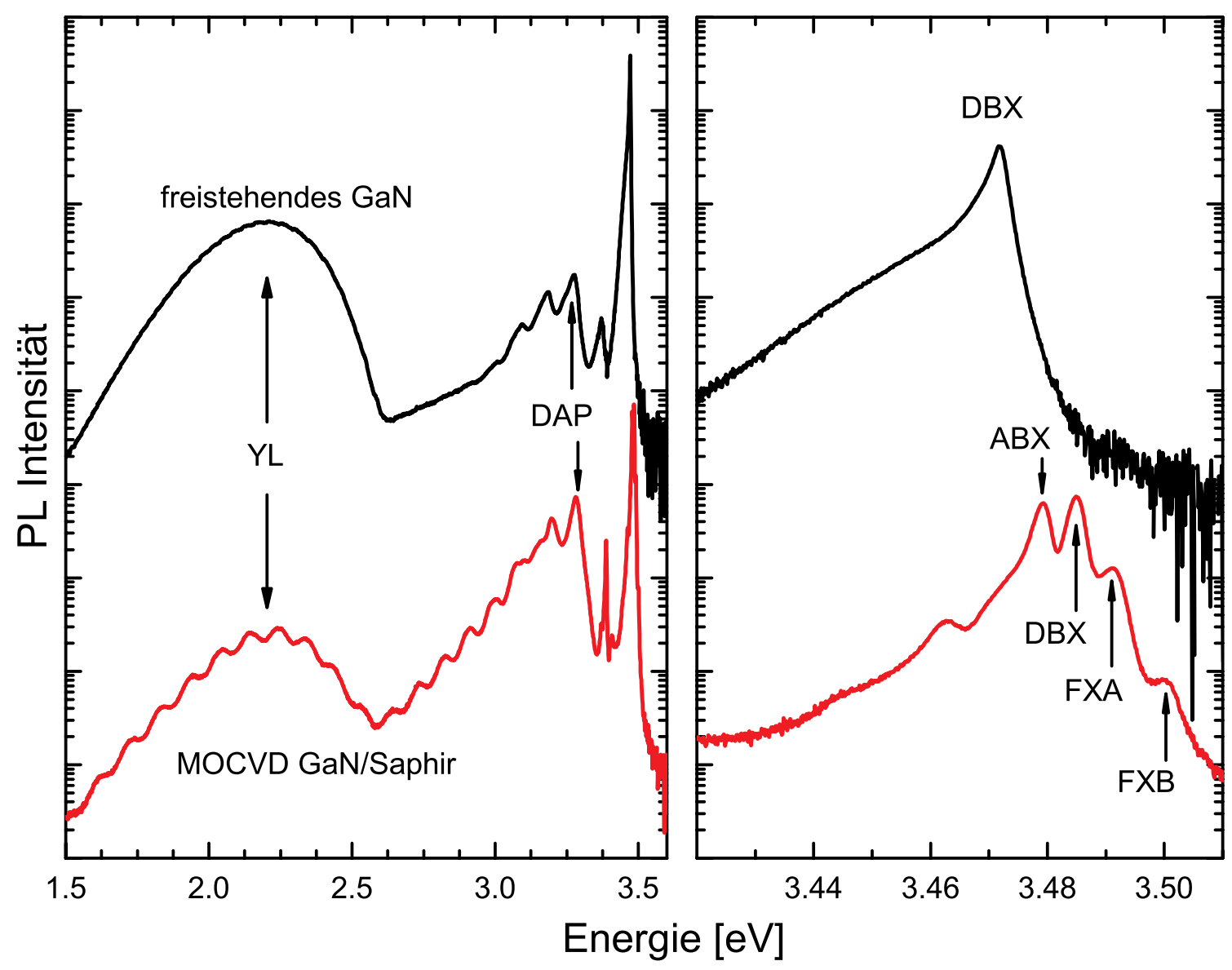

Abbildung 6.1: PL-Spektren von zwei GaN-Referenzproben. In beiden sind die gelbe Lumineszenz (YL) sowie Donator-Akzeptor-Paar Übergänge (DAP) in den Übersichtsspektren sichtbar (links). Rechts in Vergrößerung die Bandkantennahe Lumineszenz, wo im freistehenden GaN nur das Donatorgebundene Exziton (DBX) sichtbar ist, während im MOCVD-gewachsenen GaN auch ein Akzeptorgebundenes (ABX) sowie die freien Exzitonen A und B (FXA, FXB) auflösbar sind. Die Spektren sind vertikal verschoben.

(bei Temperaturen des flüssigen Heliums) lange genug existieren um strahlend zu rekombinieren, bevor sie einen gebundenen Zustand mit einer Störstelle eingehen können. Das Fehlen dieser Linien im freistehenden GaN ist ein Ausdruck der niedrigen Kristallqualität. Die hohe Donator-Konzentration, die durch die starke DBX-Linie nahegelegt wird, äußert sich beispielsweise in Raman-Messungen durch eine starke Phonon-Plasmon-Kopplung aus der eine Ladungsträgerdichte im mittleren $10^{18} \mathrm{~cm}^{-3}$-Bereich ermittelt werden kann. Ein weiteres Maß für die Kristallqualität ist die Breite der exzitonischen Lumineszenzen. Im vorliegenden Fall liegt diese für die MOCVD-Schichten bei etwa 2-4 meV sowohl für gebundene als auch freie Exzitonen. Dies ist einerseits als Benchmark für die darauf gewachsenen MBE-Schichten wichtig und erlaubt andererseits Vergleiche mit der Literatur. Linienbreiten von etwa $2 \mathrm{meV}$ gelten als sehr gut [68], während bei Homoepitaxie auf GaN-Volumenmaterial Breiten von $0.1 \mathrm{meV}$ für das DBX und etwa $2 \mathrm{meV}$ für freie Exzitonen erreicht wurden [127, 128]. 


\subsubsection{Abhängigkeit von den Wachstumsparametern}

Das Anbieten von Mn hat Auswirkungen auf den Wachstumsprozess von GaN, die sich auch in den optischen Eigenschaften äußern. Daher sind Mn- und Ga-Angebot kritische Parameter für die optischen Eigenschaften der gewachsenen Schichten. Außerdem wurden im Rahmen dieser Arbeit Proben mit verschiedenen Wachstumstemperaturen untersucht, da diese Temperatur ebenfalls vielfältige Auswirkungen auf die Lumineszenz hat. Abbildung 6.2 zeigt Spektren von GaMnN-Proben, die bei hohen und niedrigen Temperaturen gewachsen wurden, zusammen mit jeweiligen Referenzproben ohne Mn. Diese sind wie alle diskutierten Proben in diesem Abschnitt auf Si(111) gewachsen.
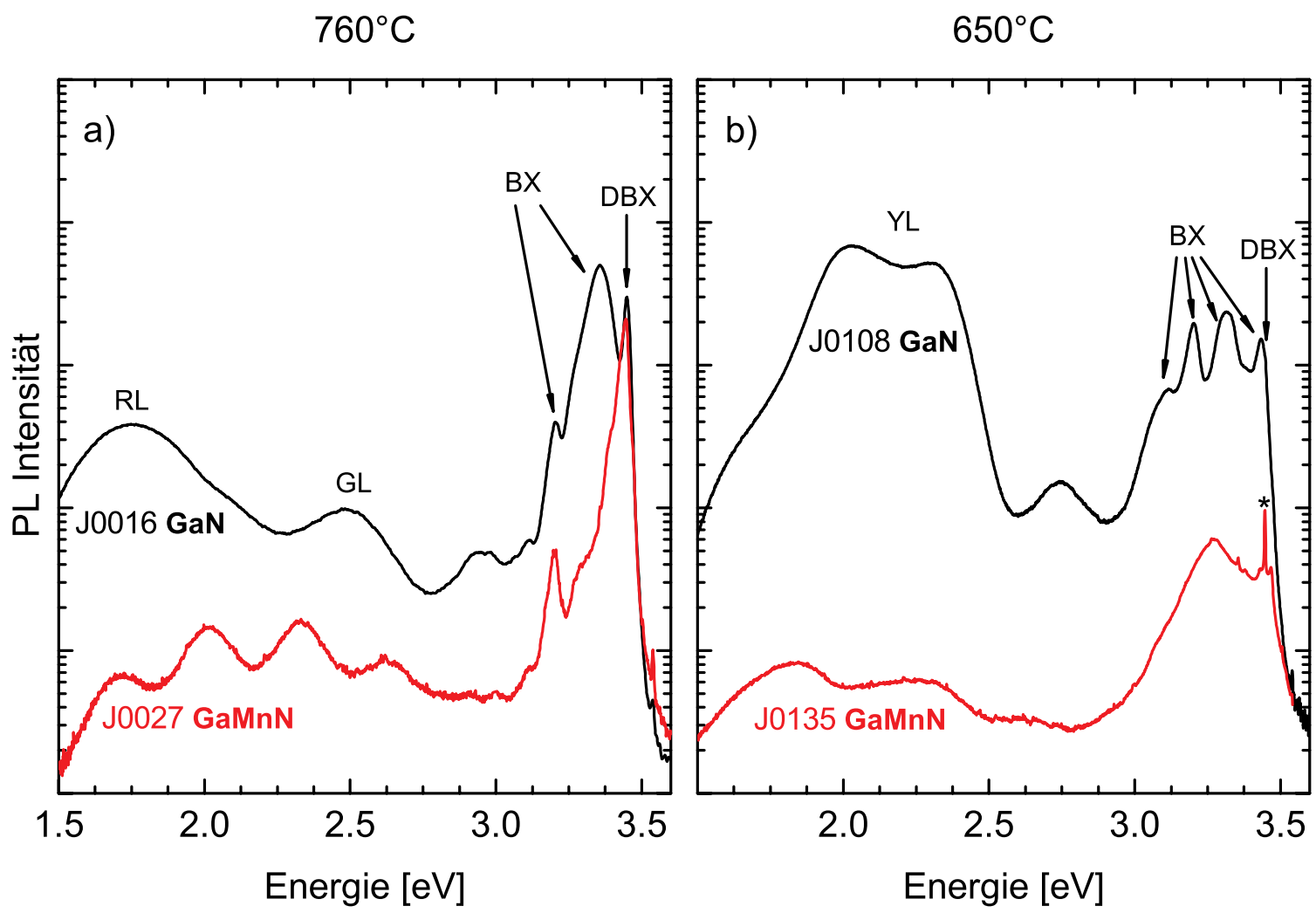

Abbildung 6.2: PL-Spektren von GaMnN bei verschiedenen Wachstumstemperaturen im Vergleich zu undotiertem GaN. Die Defektlumineszenzen im sichtbaren Bereich werden sowohl bei $760^{\circ} \mathrm{C}$ (a) als auch bei $650^{\circ} \mathrm{C}$ (b) durch den Mn-Einbau geschwächt. Die exzitonischen Lumineszenzen im UV-Bereich sind bei hohen Wachstumstemperaturen auch in GaMnN noch gut detektierbar, bei tiefen hingegen stark abgeschwächt. „*“ (in b) bezeichnet eine Phononenreplik der Laserlinie, keine Lumineszenzlinie.

Wachstumstemperatur Bei hohen Wachstumstemperaturen ist die Kristallqualität vergleichsweise hoch, wie auch im PL-Spektrum der Referenzprobe ersichtlich ist. Das Donator-gebundene Exziton (DBX) ist klar erkennbar, auch wenn es mit einer FWHM von $23 \mathrm{meV}$ relativ stark verbreitert ist. Typische Werte für GaN auf $\mathrm{Si}(111)$ liegen um $10 \mathrm{meV}$ (siehe zum Beispiel [68] und dortige Referenzen), aber auch $1.7 \mathrm{meV}$ wurden 
erzielt [130]. Des weiteren sind tiefer gebundene Exzitonen bei $3.36 \mathrm{eV}$ und $3.20 \mathrm{eV}$ sichtbar. Sie werden Exzitonen zugeordnet, die an strukturelle Defekte gebunden sind [73], den so genannten $Y_{i}$-Linien. Die typische gelbe Lumineszenz (YL) von GaN ist in diesem Fall durch zwei Bänder bei $1.8 \mathrm{eV}(\mathrm{RL})$ und $2.5 \mathrm{eV}$ (GL) ersetzt, was auf die Metall-reichen Wachstumsbedingungen zurückgeführt werden kann [73]. GaMnN bei gleicher Temperatur und sonst ähnlichen Bedingungen zeigt signifikante Abweichungen. Das DBX ist deutlich breiter (FWHM $30 \mathrm{meV}$ ). Alle anderen Lumineszenz-Signale sind stark abgeschwächt, was entweder auf eine Reduzierung der zu Grunde liegenden Defekte hindeutet oder auf die Kreation nicht-strahlender Rekombinationskanäle für diese Übergänge durch zusätzliche Defekte.

Bei tiefen Wachstumstemperaturen wurde bereits in Kapitel 5.2 gezeigt, dass die Kristallqualität abnimmt. Diese Aussage wird durch die PL-Messungen gestützt. Nahe der Bandkante ist in der Referenzprobe das DBX nur noch als schwache Schulter zu anderen BX-Signalen erkennbar, die alle strukturellen Defekten zugeordnet werden. Des weiteren ist die YL zum dominierenden Signal geworden, was allgemein als Merkmal schlechter Qualität gilt, da die YL eine Defektlumineszenz ist, die mit Ga-Vakanzen und anderen Defekten in Verbindung gebracht wird. GaMnN bei dieser Temperatur zeigt ein noch drastischeres Verhalten als bei hohen Temperaturen. Fast über den kompletten Spektralbereich ist eine starke Abschwächung beobachtbar. Nur nahe der Bandkante im Bereich der strukturell gebundenen Exzitonen ist noch eine Restlumineszenz erkennbar. Dafür tauchen nahe der Bandkante mehrere scharfe Signale auf, das stärkste bei $3.447 \mathrm{eV}$ ist in Abbildung 6.2b) mit einem "*“ markiert.

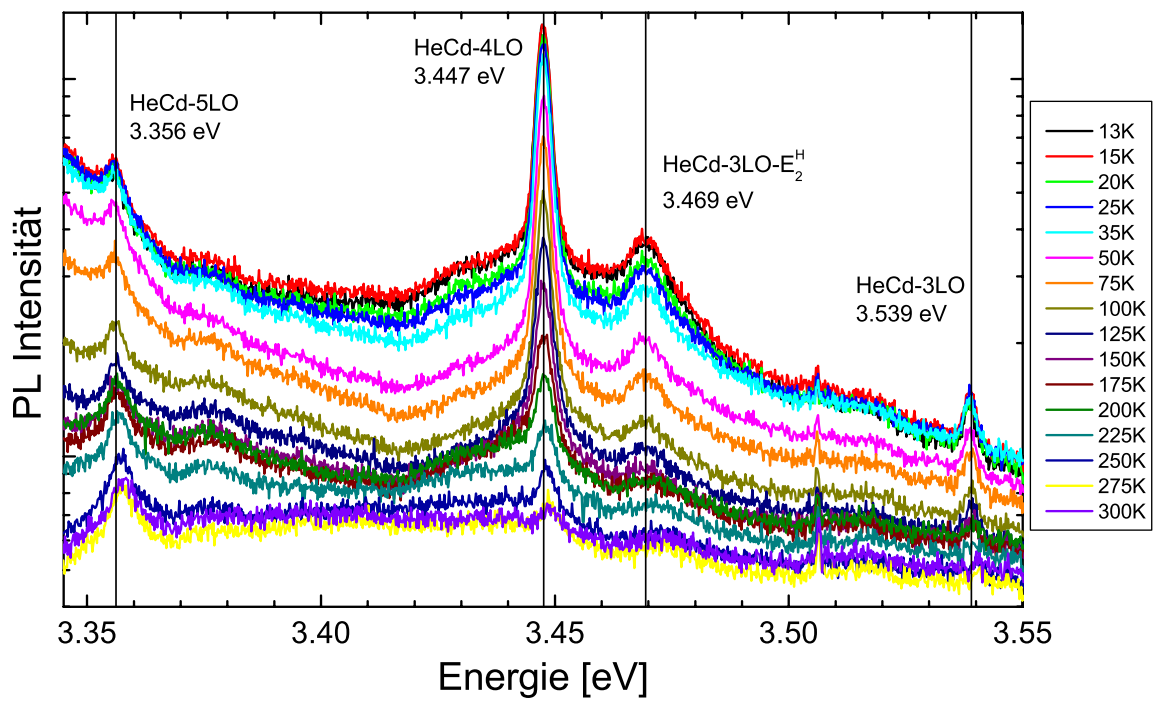

Abbildung 6.3: PL-Spektren einer Probe mit „Raman-Linien“ nahe der Bandlücke bei verschiedenen Temperaturen.

In temperaturabhängigen Messungen (Abbildung 6.3) zeigen diese Signale im Rahmen der Messgenauigkeit praktisch keine Veränderung der Position mit der Temperatur, was eine Lumineszenz ausschließt, die mit der Bandlücke von GaN zusammenhängt. Allerdings zeigt sich eine Veränderung der relativen Intensitäten dieser Signale, bei hohen 
Temperaturen dominieren die energetisch niedriger liegenden. Diese Veränderung korreliert ungefähr mit der Änderung der Bandlücke von GaN. Leistungsabhängige Messungen zeigen keinerlei Abhängigkeit dieser Linien von der Anregungsleistung außer einer linearen Intensitätsabhängigkeit. Aus diesen Informationen kann nur ein Schluss gezogen werden: Es handelt sich nicht um Lumineszenz der GaN-Probe, hängt aber trotzdem mit der Bandlücke von GaN zusammen, wie die Temperaturabhängigkeit der Intensität nahelegt. Die stärksten dieser Linien liegen bei $3.356 \mathrm{eV}, 3.447 \mathrm{eV}$ und $3.539 \mathrm{eV}$ (oberhalb der Bandlücke von GaN!), unterscheiden sich also jeweils genau um eine (GaN-)LO-PhononEnergie von $91 \mathrm{meV}$. Verlängert man diese Reihe weiter zu höheren Energien, so trifft man genau die HeCd-Laserlinie bei $3.813 \mathrm{eV}$, die zur Anregung der Lumineszenz benutzt wird. Auch die Linie bei $3.469 \mathrm{eV}$ kann mit Hilfe eines $E_{2}^{H}$-Phonons erklärt werden. Die scharfen Linien sind demnach direkte Phononenrepliken der Laserlinie, entsprechend einem Raman-Prozess höherer Ordnung. Nahe der Bandkante wird dieser Prozess resonant verstärkt, so dass diese Linien deutlich detektierbar sind, wenn die Lumineszenz in diesem Bereich schwach genug ist. Der Resonanzprozess erklärt auch den Zusammenhang der Intensität mit der Bandlücke. Ähnliche Signale wurden auch von Godlewski et al. beobachtet [131]. Dort wurde hochdotiertes GaN:Mg untersucht und mittels Mikro-PL eine Korrelation dieser Linien mit Unordnungseffekten gezeigt. Außerdem wird neben einem resonanten Raman-Prozess eine so genannte heiße Lumineszenz als Erklärung diskutiert. Welche dieser beiden Erklärungen korrekt ist, spielt für die Experimente im Rahmen dieser Arbeit keine Rolle, sie werden im Folgenden kurz mit Raman-Linien bezeichnet. Allerdings zeigt der Zusammenhang mit hohen Dotierungen, egal ob Mn oder Mg, dass das Auftreten dieser Signale typisch für Unordnung ist.

Mn-Angebot Wie bereits in Kapitel 5 diskutiert wurden bei der niedrigen Wachstumstemperatur von $650^{\circ} \mathrm{C}$ ausführliche Studien mit unterschiedlichen Ga- und MnAngeboten durchgeführt. Innerhalb des gefundenen Wachstumsfensters, wo GaMnN ohne Ausscheidungen gewachsen werden kann, wurde daher eine Serie von hochverdünnten Proben bis zu hochkonzentrierten untersucht. Die erhaltenen PL-Spektren sind in Abbildung 6.4 dargestellt.

Bei niedrigem Mn-Angebot zeigen sich unter diesen stickstoffreichen Wachstumsbedingungen keine signifikanten Auswirkungen auf die Lumineszenz. Sowohl die Referenzprobe als auch die hochverdünnte Probe zeigen eine bandkantennahe Lumineszenz von strukturell gebundenen Exzitonen als auch eine ausgeprägte gelbe Lumineszenz. Die unterschiedlichen Signale der BX hängen empfindlich von der Messposition auf der Probe ab und zeigen starke laterale Variationen. Eventuelle Unterschiede der Spektren in diesem Bereich sind also nicht aussagekräftig für die Proben sondern zeigen nur die strukturellen Defekte in einem lokalen Bereich an. Die starke YL ist eine Folge der niedrigen Wachstumstemperatur und der stickstoffreichen Wachstumsbedingungen, die die Bildung von Ga-Vakanzen fördern [119, 120].

Wird das Mn-Angebot erhöht (bei ansonsten identischen Bedingungen), so wird zuerst die bandkantennahe Lumineszenz stark unterdrückt. Bis auf ein schwaches BX-Signal bei $3.20 \mathrm{eV}$ ist sie in Probe J0146 nicht mehr nachweisbar, oberhalb dominieren bereits die Raman-Linien. Die YL ist zwar nach wie vor in relativ hoher Intensität vorhanden, 


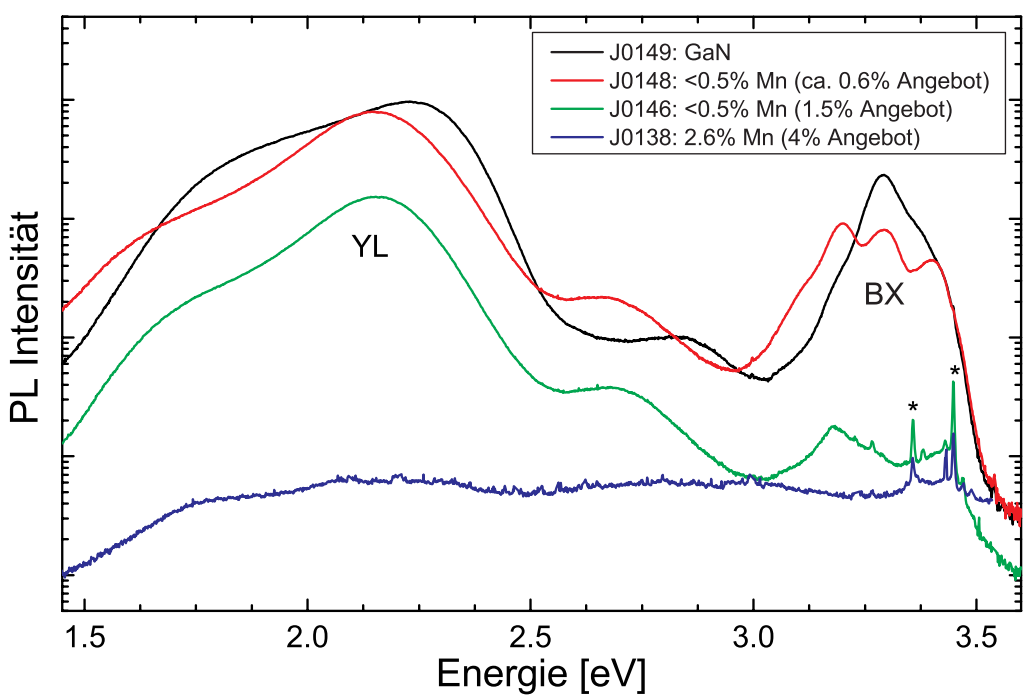

Abbildung 6.4: PL-Spektren bei Veränderung des Mn-Angebotes von Proben innerhalb des Wachstumsfensters bei tiefen Temperaturen. Im sichtbaren bis ultravioletten ist bei hohen Mn-Konzentrationen keinerlei Lumineszenz detektierbar, nur die mit „*“ markierten Raman-Linien nahe der Bandkante.

im Vergleich zu schwach- und undotierten Proben aber bereits um eine Größenordnung unterdrückt. Dotierungen im Prozent-Bereich führen zu einem kompletten Verschwinden jeglicher Lumineszenz. Für diesen massiven Effekt von mehr als drei Größenordnungen müssen hohe Konzentrationen an nicht-radiativen Defekten erzeugt werden, beispielsweise durch tiefe Störstellen.

\subsubsection{Abhängigkeit vom Substrattyp}

Die strukturellen Charakterisierungen (siehe Kapitel 5.2.3) zeigten eine starke Verbesserung der Kristallqualität bei Verwendung von Saphir-Substrat mit einer MOCVDgewachsenen GaN-Pufferschicht anhand von Röntgenuntersuchungen. PhotolumineszenzMessungen reagieren ebenfalls sehr empfindlich auf die Kristallqualität und die Auswirkungen sind hier tatsächlich noch deutlicher.

In Abbildung 6.5a) sind zwei GaN-Referenzproben auf beiden Substraten verglichen. Zeigen die tiefen Störstellen (verantwortlich für gelbe und rote Lumineszenz) noch ein vergleichbares Verhalten, so sind nahe der Bandkante qualitative Unterschiede erkennbar. Anstelle breiter Bänder von Exzitonen, die an strukturelle Defekte gebunden sind, treten hier viele sehr scharfe Linien auf. In Teil b) der Abbildung ist hiervon eine Vergrößerung nahe der Bandkante zu sehen. Im Wesentlichen sind die gleichen Linien wie in der unbehandelten Pufferschicht (siehe Abbildung 6.1) detektierbar. Ein zusätzliches Signal befindet sich $20 \pm 1 \mathrm{meV}$ oberhalb des FXA-Übergangs und kann einem angeregten Zustand dieses freien Exzitons zugeordnet werden. Unter Annahme eines WasserstoffModells für das Exziton ergibt sich damit für das Exziton A nach Gleichung 4.3 eine Bindungsenergie von $E_{X}=4 / 3\left(E_{n=2}-E_{n=1}\right)=4 / 3 \cdot 20 \mathrm{meV} \approx 27 \pm 1 \mathrm{meV}$, was sehr gut mit den Werten übereinstimmt, die mit Hilfe der Temperaturabhängigkeit der 

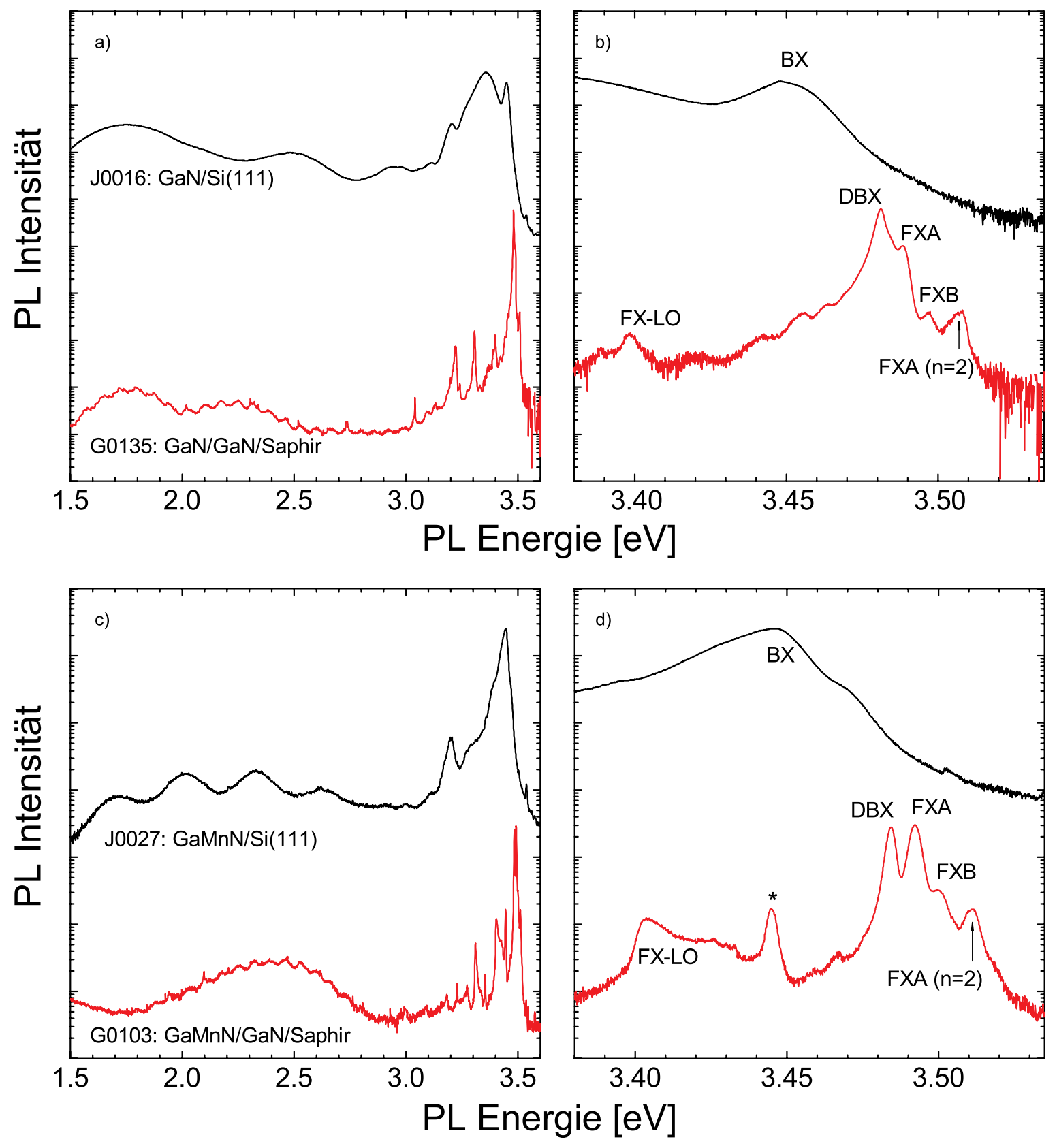

Abbildung 6.5: Vergleich von GaN-Referenzproben auf Si(111) und Saphir mit GaN-Pufferschicht im (a) kompletten Spektrum und (b) vergrößert nahe der Bandkante. (c) und (d) zeigen identische Messungen an GaMnN. Die GaN/Si-Kurven entsprechen den bereits in Abbildung 6.2a) gezeigten. 
FXA-Linie errechnet wurden $(26.7 \pm 0.5 \mathrm{meV}$ von Volm et al. [71]). Alle exzitonischen Linienbreiten sind bei verschiedenen MOCVD-Puffern und jeweils darauf gewachsenen MBE-GaN-Schichten im Rahmen der Messgenauigkeit gleich und liegen bei $2-4 \mathrm{meV}$. Das Fehlen von Akzeptor-gebundenen Exzitonen oder DAP-Übergängen zeigt außerdem die Abwesenheit von unerwünschten Akzeptoren im intrinsischen Material. In Übereinstimmung mit den strukturellen Untersuchungen zeigt die PL also auch die wesentlich erhöhte Kristallqualität von GaN auf Saphir-Substrat mit GaN-Pufferschicht. Auch die laut XRD übereinstimmende Qualität des MOCVD-Puffers mit der MBE-Schicht ist aus den exzitonischen Linienbreiten ablesbar.

In Abbildung 6.5c) und d) sind entsprechende Messungen an GaMnN gezeigt. Die Mn-Konzentration liegt in beiden Fällen bei etwa 0.5\%. Die bereits in Kapitel 6.1.1 angesprochenen Effekte wie eine Abnahme der Intensität im gesamten Spektralbereich ist auch in den hochqualitativen Proben auf Saphir-Substrat zu beobachten. Nahe der Bandkante sind auf Grund der scharfen Linien aber noch mehr Details erkennbar. Bereits bei diesen niedrigen Konzentrationen sind die Phononenrepliken der Laserlinien (,* $\left.{ }^{*}\right)$ beobachtbar, die auf Unordnung hinweisen. Die exzitonischen Linien zeigen im Vergleich zur Referenzproben keine Verbreiterung, was auf nach wie vor unverändert hohe Kristallqualität schließen lässt. Das Verhalten dieser exzitonischen Übergänge soll im nächsten Abschnitt genauer untersucht werden.

\subsubsection{Kompensierende Wirkung von $\mathrm{Mn}$ in GaMnN}

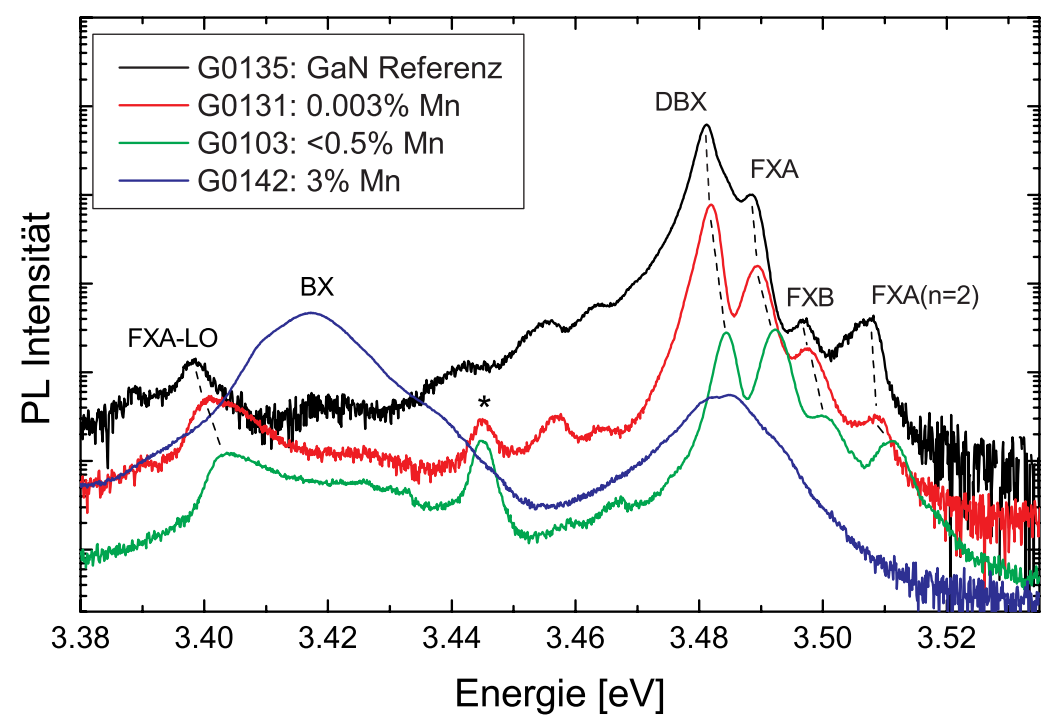

Abbildung 6.6: GaMnN mit unterschiedlichem Mn-Gehalt bei gleichen Messbedingungen. Mn schwächt die Lumineszenz und führt im Prozentbereich zu starken Verbreiterungen und strukturellen Defekten.

In Abbildung 6.6 ist die exzitonische Lumineszenz für verschiedene MnKonzentrationen abgebildet. Schon bei den kleinsten erreichten Dotierkonzentrationen 
von $0.003 \%$ (laut SIMS, entsprechend etwa $10^{18} \mathrm{~cm}^{-3}$ ) ist eine signifikante Abnahme der Intensität feststellbar. Bis zu einer Konzentration von etwa $0.5 \%$ setzt sich dieser Trend monoton fort, die Spektren bleiben aber qualitativ gleich. Oberhalb etwa 1\% MnGehalt sind keine scharfen Linien der freien und gebundenen Exzitonen mehr sichtbar, sondern nur noch ein einziges, stark verbreitertes DBX-Signal mit mehr als $10 \mathrm{meV}$ Halbwertsbreite. Dieses Verhalten konnte an Hand mehrerer Proben verifiziert werden. Die starke Verbreiterung zusammen mit dem Verschwinden der freien Exzitonen deutet auf deutlich defektreichere Proben hin. Gleichzeitig sind in genau diesen Proben strukturellgebundene Exzitonen in hoher Intensität nachweisbar. Die hochkonzentrierten und defektreichen Proben zeigen außerdem eine gelblich-bräunliche Farbe im Vergleich zu den transparenten verdünnten GaMnN- und GaN-Proben. Die GaMnN-Proben müssen also in zwei gesonderten Kategorien betrachtet werden, zum Einen die optisch hochqualitativen verdünnten Schichten und zum Anderen die hochkonzentrierten Schichten mit schlechter optischer Qualität. Eine Probe (G0105), welche sowohl braune als auch transparente Bereiche aufwies, zeigte in jedem dieser Bereiche genau die gerade beschriebenen typischen Signaturen.

Weiterhin kann man Abbildung 6.6 entnehmen, dass mit zunehmendem Mn-Gehalt sich das Verhältnis der freien zu gebundenen Exzitonen ändert. In Referenzschichten ist das DBX-Signal das klar dominierende, es wird allerdings mit zunehmendem Mn-Einbau deutlich stärker als die FX-Lumineszenz unterdrückt. Hier zeigt sich der kompensierende Einfluss von Mn als tiefer Störstelle. In der Schicht vorhandene flache Donatoren werden entladen und gehen damit in den positiven Ladungszustand über. Die DBX-Lumineszenz ist dem neutralen Donator zugeordnet, der dementsprechend geschwächt wird. Allerdings konnten keine Übergänge nachgewiesen werden, die einem geladenen Donator oder Akzeptor-gebundenen Exziton zugeordnet werden könnten. Zur genaueren Analyse dieser Hypothese wurden daher leistungsabhängige Messungen durchgeführt.

In Abbildung 6.7 ist das Ergebnis dargestellt. Es zeigt sich für alle exzitonischen Linien ein schwach superlineares Verhalten. Für freie Exzitonen ist dieses Ergebnis leicht nach vollziehbar, da sie als Rekombinationspfad keinerlei Sättigungsprozessen unterworfen sind. Erst bei extrem hohen Leistungsdichten, wenn man sich einem Elektron-LochPlasma nähert, werden die exzitonischen Rekombinationen abgeschwächt. Dies ist aber erst im Bereich $1 \mathrm{MW} / \mathrm{cm}^{2}$ zu erwarten [126], drei Größenordnungen oberhalb der hier verwendeten Anregungsdichten. Demgegenüber sind die Konkurrenzprozesse wie etwa die defektinduzierten Lumineszenzen und auch nicht-strahlende Kanäle durch die Anzahl der verursachenden Defekte und der entsprechenden Lebensdauern limitiert, so dass für freie Exzitonen bei niedrigen bis mittleren Anregungsleistungen ein superlineares Verhalten erwartet werden kann.

Bei gebundenen Exzitonen ist die gleiche Argumentation gültig, so lange der Defekt an den sie gebunden sind in ausreichend hoher Konzentration vorhanden ist. Sobald diese Defekte alle „besetzt“ sind, sollte das super- in eine sublineares Verhalten umschlagen. Das konnte hier allerdings nicht beobachtet werden. Im Gegenteil, das DBX zeigt sogar einen noch stärkeren Anstieg als die freien Exzitonen, ein auf den ersten Blick widersprüchliches Ergebnis. Obwohl tatsächlich die Zahl der Donatoren mit der Anregungsleistung nicht zunehmen kann, trifft diese Feststellung nicht auf die Zahl der Donatoren 


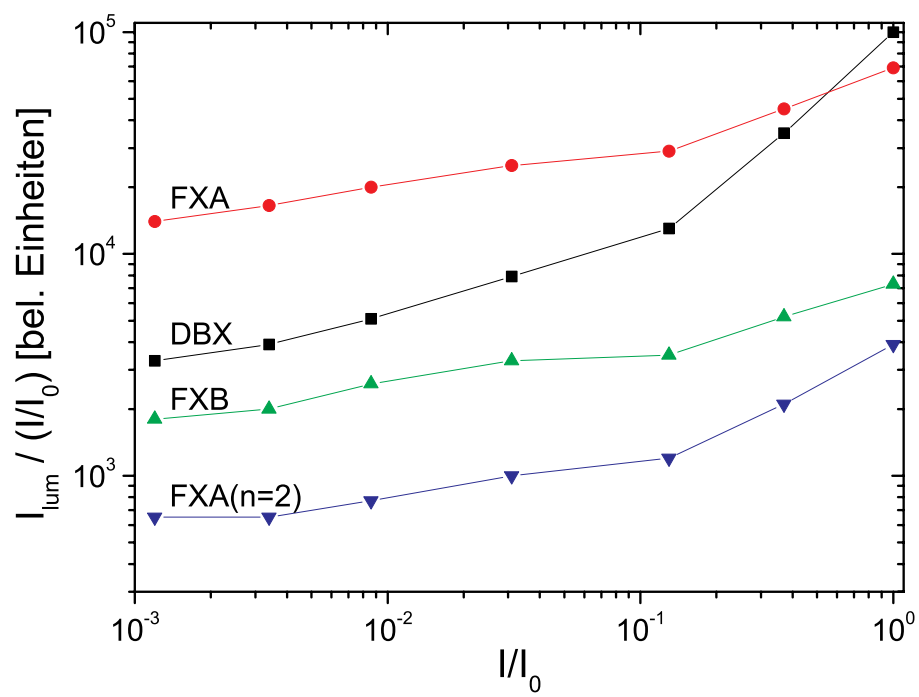

Abbildung 6.7: Leistungsabhängigkeit der exzitonischen Übergänge in GaMnN. Die PL-Intensitäten ( $\mathrm{I}_{\text {lum }}$ ) wurden auf die Anregungsleistung $I$ normiert, so dass eine Waagerechte einem linearen Verhalten entspräche. Die maximale Anregungsleistung $I_{0}$ beträgt etwa $1 \mathrm{~kW} / \mathrm{cm}^{2}$.

im neutralen Ladungszustand zu, und nur diese tragen zur DBX-Lumineszenz bei. Wenn ein erheblicher Anteil der Donatoren positiv geladen ist, so können diese geladenen Donatoren mit zunehmender Laserleistung (und zunehmender Konzentration an erzeugten Elektronen-Loch-Paaren) Elektronen einfangen und dadurch in den neutralen Ladungszustand übergehen. Bevor dieses Elektron wieder mit einem Loch rekombiniert, kann ein Exziton gebunden werden, was seinerseits strahlend zerfällt. Durch diese zweifache Abhängigkeit von der eingestrahlten Leistung ist in einem kompensierten System das stark superlineare Verhalten der DBX-Lumineszenz erklärbar.

\section{Zusammenfassung}

In diesem Kapitel wurde gezeigt, dass die PL-Messungen klar mit den XRD-Messungen korrelieren, was die Kristallqualität angeht. Es lassen sich allerdings deutlich feinere Details an den exzitonischen Lumineszenzen erkennen, die eine Rückkopplung für das Optimieren des Wachstumsprozesses ermöglichen. Außerdem wird bei Probendicken von mehreren hundert Nanometern kein Signal des GaN-Puffers mitgemessen, welches bei Röntgenmessungen eine Analyse erschwert. Die Eigenschaft von Mn, jegliche Lumineszenz in GaN zu unterdrücken, wurde an Hand vieler Proben klar dokumentiert. Allerdings bleiben die exzitonischen Linienbreiten und damit die optische Qualität der Schichten auf SaphirSubstrat bis etwa 1\% Mn-Gehalt auf dem hohen Niveau der MOCVD-Pufferschicht. Es konnte außerdem ein kompensierender Einfluss von Mn auf das im intrinsichen Fall nleitende GaN festgestellt werden, was die Eigenschaft als tiefer Akzeptor bestätigt. Dies ist in Übereinstimmung mit elektrischen Messungen, in denen die GaMnN-Schichten hochohmig waren. 


\subsection{PL im Infraroten}

Reines GaN zeigt im Infraroten keine Lumineszenz. In diesem Spektralbereich sind höchstens noch Schultern der gelben Lumineszenz detektierbar, um so stärker, wenn sich die gelbe zur roten Lumineszenz verändert hat. Allerdings treten in diesem Spektralbereich bevorzugt die intra-3d-Lumineszenzen der Übergangsmetalle auf (siehe Kapitel 2.2.1), sowie intra-4f-Lumineszenzen einiger seltenen Erden. Beispielsweise ist bei $1.30 \mathrm{eV}$ ein interner Übergang von $\mathrm{Fe}^{3+}$ [132] in nominell undotierten MOCVD-GaN-Schichten zu beobachten.

\subsubsection{Identifikation des optischen Übergangs bei $1.4 \mathrm{eV}$}

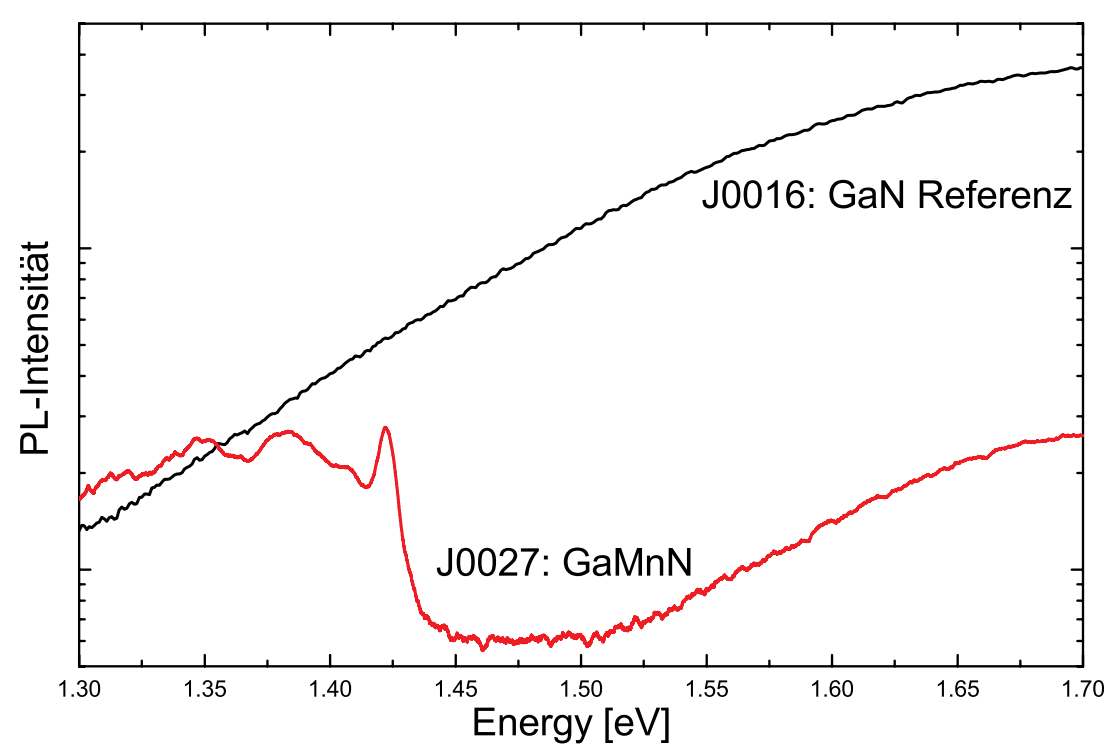

Abbildung 6.8: PL im Infraroten an Probe J0027 und einer GaN-Referenzprobe. Ein neues Signal bei $1.4 \mathrm{eV}$ ist in der GaMnN-Probe sichtbar.

Abbildung 6.8 zeigt PL-Messungen im IR-Bereich. Während die Referenzprobe keine Signale außer der breiten Schulter der roten Lumineszenz zeigt, findet man bei bei GaMnN eine auffällige Lumineszenz bei etwa $1.4 \mathrm{eV}$. Sie besteht aus einer relativ scharfen Linie bei $1.42 \mathrm{eV}$ und einem breiten, strukturierten Band auf der niederenergetischen Seite. Die Ursache dieser Lumineszenz und des zu Grunde liegenden physikalischen Prozesses soll im Folgenden analysiert werden.

\subsubsection{Temperaturabhängigkeit}

Einen wichtigen Hinweis auf den physikalischen Ursprung einer Lumineszenz liefern temperaturabhängige Messungen. Diese konnten mit dem Standard-PL-Aufbau nicht durch- 
geführt werden, da im Badkryostaten die Temperatur durch das flüssige Helium fest vorgegeben ist. Daher wurden sie an einem ähnlichen Aufbau im II. Physikalischen Institut der Universität Göttingen durchgeführt, bei dem die Probe über ein „,closed cycle“-System im Vakuum bis auf etwa $15 \mathrm{~K}$ gekühlt und durch eine Gegenheizung kontinuierlich bis $300 K$ erhitzt werden kann. Die Anregung erfolgt ebenfalls mit einem HeCd-Laser, das Spektrometer wurde mit einem Gitter mit 100 Linien/mm bestückt. Die Detektion erfolgt mit einer CCD-Kamera. Details zu diesem Aufbau sind beispielsweise in [133] und [134] zu finden.

Da dieser Aufbau nicht für den Spektralbereich um $900 \mathrm{~nm}$ optimiert ist, traten Schwierigkeiten auf, deren Lösung im Anhang beschrieben ist.

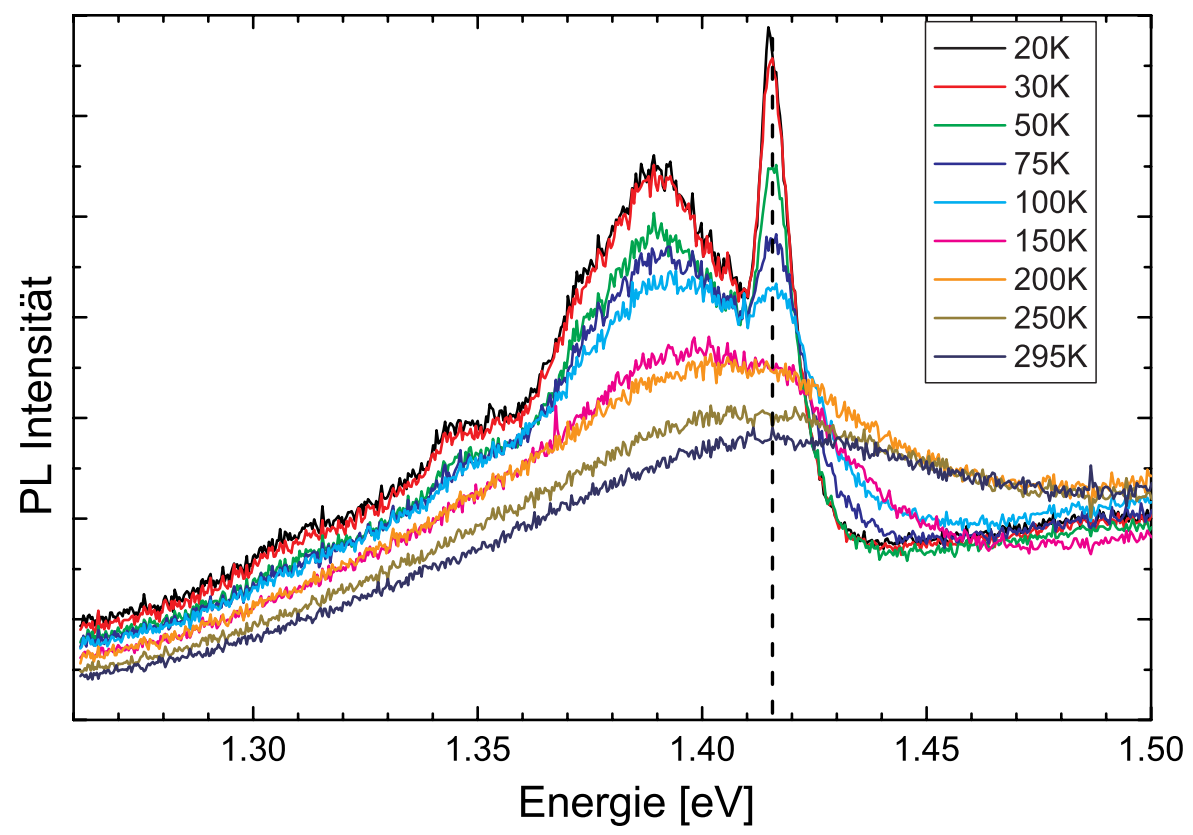

Abbildung 6.9: Temperaturabhängigkeit der Lumineszenz bei $1.4 \mathrm{eV}$ von Probe J0080. Die scharfe Linie bei $1.42 \mathrm{eV}$ verschwindet bei $100 \mathrm{~K}$, das breite Band auf der niederenergetischen Seite verschiebt sich zu höheren Energien.

In Abbildung 6.9 ist die Temperaturabhängigkeit von Probe J0080 zu sehen, die eine vergleichsweise starke Linie bei $1.42 \mathrm{eV}$ aufweist. Andere Proben zeigen qualitativ das gleiche Verhalten. Die scharfe Linie bei $1.42 \mathrm{eV}$ ändert ihre energetische Position nicht mit steigender Temperatur (im Rahmen der Messgenauigkeit von $\approx 1 \mathrm{meV}$ ), wird aber schwächer bis sie bei etwa $100 K$ verschwindet. Das Seitenband verliert bei ungefähr der gleichen Temperatur seine Struktur und fängt an, sich zu höheren Energie zu verschieben. Bei Raumtemperatur liegt es symmetrisch mit seinem Zentrum nahe der ursprünglichen Position der scharfen Linie.

Dieses Verhalten passt zu einem Übergang mit einer Energie von $1.42 \mathrm{eV}$, der von einem phononischen Seitenband begleitet ist. Bei tiefen Temperaturen relaxiert das angeregte Niveau dieses Übergangs sofort in seinen (vibronischen) Grundzustand, die höchste auftretende Photonen-Energie entspricht einem Übergang zum Grundzustand des unteren Niveaus. Gleichzeitig können aber auch nach dem Franck-Condon-Prinzip (siehe Kapitel 
2.2.3) Phononen emittiert werden, die die Photonenenergie absenken. Bei hohen Temperaturen ist auch das obere Niveau vibronisch angeregt und beim Übergang können die Phononen sowohl annihiliert als auch erzeugt werden. Das Spektrum wird damit nahezu symmetrisch zur Hauptlinie, im folgenden kurz ZPL (zero phonon line) genannt.

Die ZPL verändert ihre Position mit der Temperatur nicht. Damit kann an diesem Übergang kein Zustand beteiligt sein, der direkt mit Valenz- oder Leitungsband korreliert ist, da deren energetische Position von $0-100 \mathrm{~K}$ sich in der Größenordnung von $10 \mathrm{meV}$ verschiebt [21, 135, 136]. Eine mögliche Erklärung wäre also ein Übergang zwischen zwei tiefen Störstellen. Allerdings ist in diesem Fall eine starke Elektron-Phonon-Kopplung zu erwarten, was ein starkes phononisches Seitenband und eine schwache, wenn überhaupt sichtbare, ZPL zur Folge hätte. Eine zweite Möglichkeit ist ein intra-3d-Übergang. Da hierbei nur ein Atom in verschiedenen elektronischen Zuständen beteiligt ist, ist hier keine direkte Verschiebung mit den Bandkanten zu erwarten. Auch erklärt es die relativ scharfe ZPL, da die natürliche Linienbreite dieser Übergänge extrem klein ist. Die experimentellen Linienbreiten werden normalerweise durch die Materialqualität limitiert, da eine inhomogene Verteilung von Verspannungen und Defekten eine lokale Änderung des Kristallfeldes hervorrufen kann, was zu einer statistischen Verbreiterung der beobachteten Linie führt.

Da die Lumineszenz bei $1.42 \mathrm{eV}$ nur in Mn-dotierten Proben auftritt, muss der zu Grunde liegende Effekt mit dem Mn zusammenhängen. Auf Grund der in diesem Kapitel dargelegten Erkenntnisse ist eine Zuordnung zu einem intra-3d-Übergang des Mn-Atom wahrscheinlich. Zumindest in den Ladungszuständen $\mathrm{Mn}^{3+}$ und $\mathrm{Mn}^{4+}$ sind spin-erlaubte Übergänge möglich, aber auch spin-verbotene Übergänge des $\mathrm{Mn}^{2+}$-Ions sind nicht auszuschließen.

\subsubsection{Einordnung in die vorhandene Literatur}

Zum Zeitpunkt der Identifikation der $1.42 \mathrm{eV}$-Bande gab es in der Literatur keinerlei Erwähnung einer vergleichbaren Lumineszenz. Allerdings werden von verschiedenen Gruppen Absorptionsmessungen berichtet [16, 137, 138, 139, 140], die bei $1.4-1.5 \mathrm{eV}$ ein Signal ergaben. Frühe Untersuchungen von Korotkov et al. [137, 38] wurden so interpretiert, dass diese Absorptionsbande auf einen Übergang vom Valenzband zum tiefen Mn-Akzeptor-Niveau zurückzuführen ist. Allerdings zeigten Graf et al. [16, 141] kurz darauf, dass der beobachtete Übergang ein intra-3d-Übergang sein musste, während der Valenzband-Akzeptor-Übergang von Mn bei $1.8 \mathrm{eV}$ beobachtbar ist [142]. Der intra-3dÜbergang wurde dabei dem spin-erlaubten ${ }^{5} T_{2} \rightarrow{ }^{5} E$ Übergang von $\mathrm{Mn}^{3+}$ zugeordnet. Das ist der nach der Kristallfeldtheorie zu erwartende Übergang vom Grundzustand in den ersten angeregten Zustand (siehe Kapitel 2.2). Diese Zuordnung wurde auch später durch magneto-optische Messungen bestätigt [35, 37], die klar einen $d^{4}$-Zustand identifizierten und eine hohe Übereinstimmung mit einem theoretischen Modell erzielten, dass genau die Eigenschaften dieses ${ }^{5} T_{2} \rightarrow{ }^{5} E$ Übergangs benutzt.

Abbildung 6.10 zeigt die PL-Messungen aus Abbildung 6.8 im direkten Vergleich mit Absorptionsdaten, die freundlicherweise von A. Wolos zur Verfügung gestellt wurden [35]. Die phononischen Seitenbänder sind bei Absorptionsmessungen bei tiefen Temperaturen 


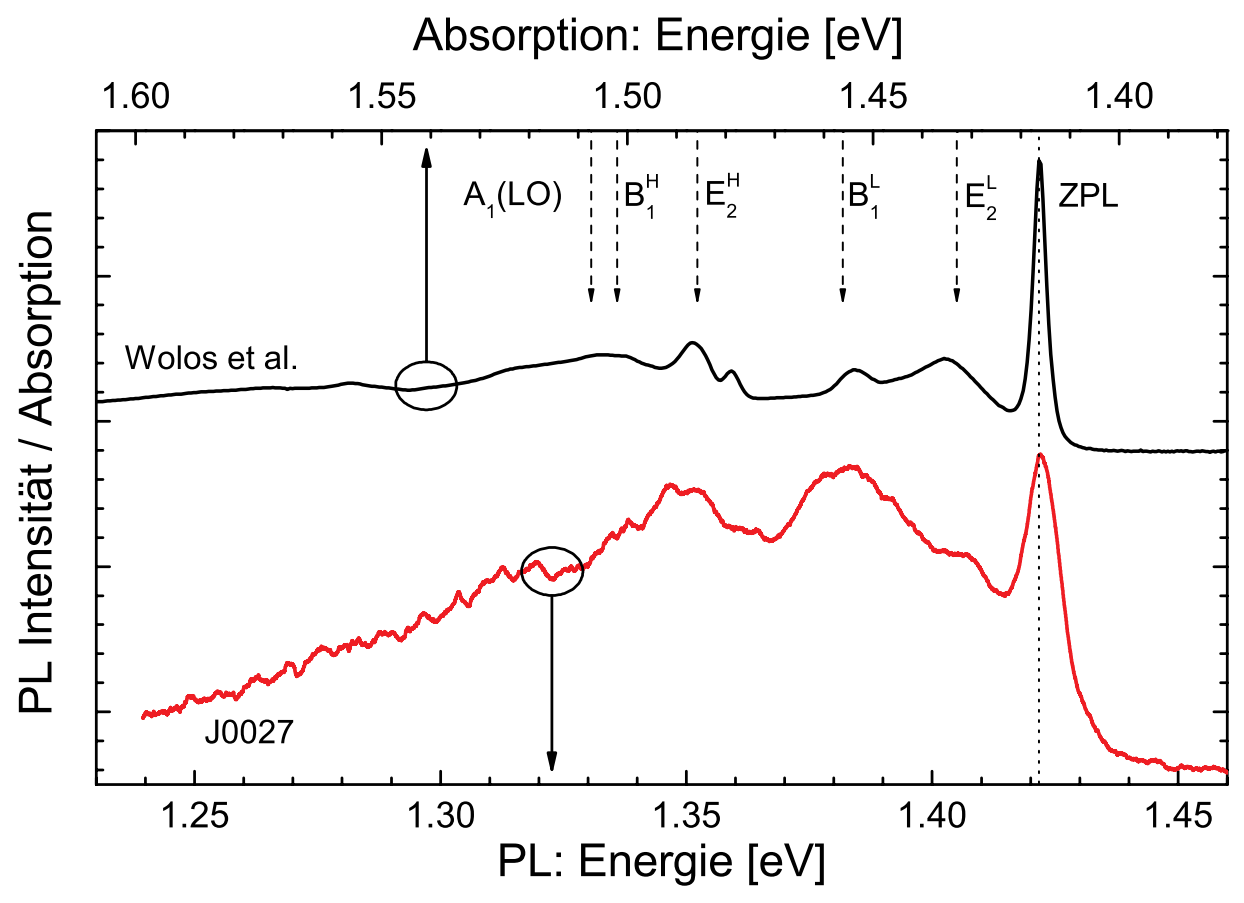

Abbildung 6.10: Direkter Vergleich einer Absorptionsmessung (schwarz, obere Skala) von Wolos et al. [35] mit der PL-Messung an Probe J0027 (rot, untere Skala). Die x-Achse wurde für die Absorptionsmessung gespiegelt und verschoben, so dass die ZPL übereinanderliegen.

auf der hochenergetischen Seite zu finden, da auch hier der Übergang vom vibronischen Grundzustand ausgeht und nach dem Franck-Condon-Prinzip eine höhere Energie notwendig ist, um einen vibronisch angeregten Zustand des höheren Niveaus zu erreichen. Aus diesem Grund wurde die Energie-Achse gespiegelt, um auch die Seitenbänder beider Messungen direkt vergleichen zu können. Die ZPL liegt bei den beiden Proben bei leicht unterschiedlichen Energien. Dies könnte auf unterschiedliche Verspannungen zurückzuführen sein, da Wolos et al. unverspanntes GaMnN-Volumenmaterial untersuchten, während die hier vorgestellte Probe auf Si-Substrat gewachsen ist und damit einer tensilen Verspannung ausgesetzt ist. Zum Ausgleich dieses Effekts ist das Absorptionsspektrum so verschoben, dass die ZPL exakt übereinander liegen. Die schmalere Linienbreite der ZPL bei der Absorptionsmessung lässt sich mit der höheren Qualität der Volumenprobe im Vergleich zur untersuchten Schicht erklären. Die Phononenrepliken haben ihre Maxima in dieser Darstellung an den gleichen Positionen. So lassen sich in beiden Messungen Maxima erkennen, deren Energiedifferenz zur ZPL sehr gut den Phononenmoden $E_{2}^{L}, B_{1}^{L}$ und $E_{2}^{H}$ in GaN entspricht. Die höherenergetischen Phononenmoden lassen sich in der Absorptionsmessung auf Grund von Überlagerungen mit der zweiten Ordnung der niederenergetischen nur noch schlecht erkennen, bei der PL ist das Rauschen der Messung zu stark für eine bessere Analyse.

Theoretische Rechnungen stützen die Zuordnung zu einem intra-3d-Übergang. Bei- 
spielsweise berechnen Kulatov et al. [143] mit der tight-binding linear-muffin-tin-orbital (TB-LMTO) Methode die elektronischen Zustandsdichten. Das Ga wird dabei in einer Superzelle mit 64 Atomen an 1 bis 8 Plätzen durch Mn ersetzt um verschiedene Konzentrationen zu simulieren. Das Ergebnis zeigt, ähnlich wie bei anderen theoretischen Untersuchungen [13], lokalisierte Zustände in der Bandlücke von GaN, die den $e$ - und $t_{2^{-}}$ Zuständen des Mn zugeordnet werden. Es wird ein Übergang bei $\approx 1.25 \mathrm{eV}$ vorhergesagt, der den experimentellen Ergebnissen entsprechen würde.

Popovic et al. [144] vergleichen mit einem ähnlichen theoretischen Ansatz den substitutionellen und interstitiellen Einbau von Mn in GaN. Auch sie sagen für den substitutionellen Fall $\mathrm{Mn}_{G a}$ einen optischen Übergang ${ }^{5} E \rightarrow{ }^{5} T_{2}$ vorher, der bei $\approx 1.4 \mathrm{eV}$ liegen soll. Das in der Oktaederlücke eingebaute interstitielle $\mathrm{Mn}_{I}$ soll demnach zumindest in Lumineszenz keine optischen Übergänge zeigen, da die angeregten Zustände hier jeweils Elektronen in Niveaus erfordern, die resonant im Leitungsband liegen. Diese angeregten Zustände können dann aber sehr effektiv nicht-radiativ rekombinieren, was im Vergleich zu dem in diesem Fall spin-verbotenen radiativen Übergang $\left(\mathrm{Mn}_{I}\right.$ ist als Doppeldonator in Form von $\mathrm{Mn}^{2+}$ als $\mathrm{d}^{5}$-Zustand vorhanden) mit seiner langen Lebensdauer die günstigere Alternative darstellt. Das Beobachten dieser Lumineszenz zeigt also direkt einen zumindest teilweisen substitutionellen Einbau an und korreliert damit mit den Ergebnissen aus Kapitel 5.3.

\subsubsection{Abhängigkeit von den Wachstumsparametern}

Intra-3d-Übergänge werden nur indirekt von der Matrix beeinflusst. Ändern sich also Eigenschaften der Matrix, so hat dies keine direkten Auswirkungen auf den Übergang. Allerdings haben Verspannungen beispielsweise Einfluss auf das Kristallfeld, da die Atomabstände geändert werden. Dies kann ebenso Auswirkungen haben wie höhere Defektdichten, die sich möglicherweise auf die lokale Umgebung des Mn-Atoms auswirken.

Bei hohen Wachstumstemperaturen ergeben sich vergleichbare Spektren, wie auch schon in Abbildung 6.8 und $6.9 \mathrm{zu}$ sehen war. Auch bei der niedrigen Wachstumstemperatur $\left(650^{\circ} \mathrm{C}\right)$ finden sich Proben, die qualitativ gleiche Lumineszenzen zeigen. Es ist also kein genereller Effekt der Wachstumstemperatur erkennbar. Bei $650^{\circ} \mathrm{C}$ wurden dann systematische Studien zum Verhalten bei Änderung des Ga- und Mn-Flusses durchgeführt.

Abbildung 6.11 zeigt das Verhalten bei Erhöhung des Mn-Anteil und gleichzeitig konstantem Gesamtmetallfluss von $1 \times 10^{-8}$ mbar, also im extrem Stickstoffreichen. In der Probe mit niedrigem Mn-Gehalt ist der intra-3d-Übergang klar zu erkennen. Mit steigender Mn-Konzentration sinkt allerdings die Intensität und bei Probe J0088 ist kein Signal bei $1.42 \mathrm{eV}$ mehr nachweisbar. Auf den ersten Blick ist dieses Resultat kontraintuitiv, kann allerdings leicht damit erklärt werden, dass sich bei hohen Konzentrationen das Mn bevorzugt in Ausscheidungen befindet und die GaN-Matrix nur geringe Mn-Konzentrationen enthält. Außerdem nimmt in dieser Serie auch die Intensität der Lumineszenz im gesamten Spektralbereich ab, was auf eine höhere Konzentration nicht-strahlender Rekombinationszentren hindeutet. Diese bilden sich beispielsweise an den Grenzflächen zu den Ausscheidungen und können so die Lumineszenz bei $1.42 \mathrm{eV}$ unterbinden.

In Abbildung 6.12 werden Proben analysiert, die bei vergleichbarem Mn-Fluss mit 


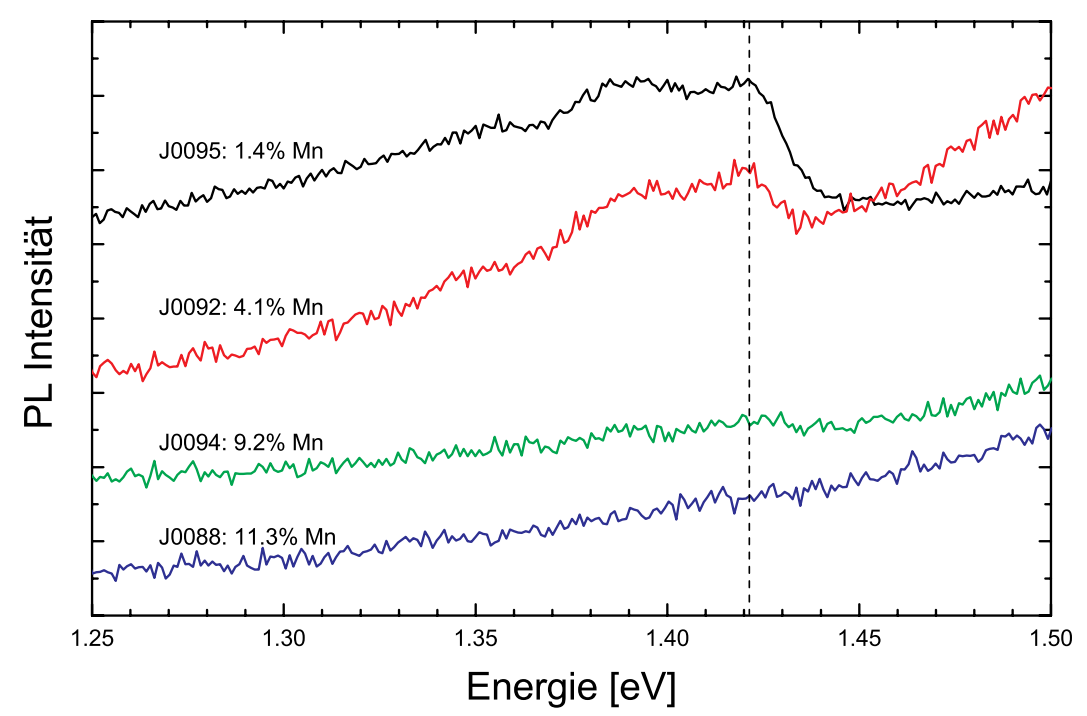

Abbildung 6.11: Verhalten der Lumineszenz bei $1.42 \mathrm{eV}$ bei Erhöhung des Mn-Angebots. Oberhalb etwa $5 \%$ eingebautes Mn verschwindet die Lumineszenz. Die Spektren sind vertikal zueinander verschoben.

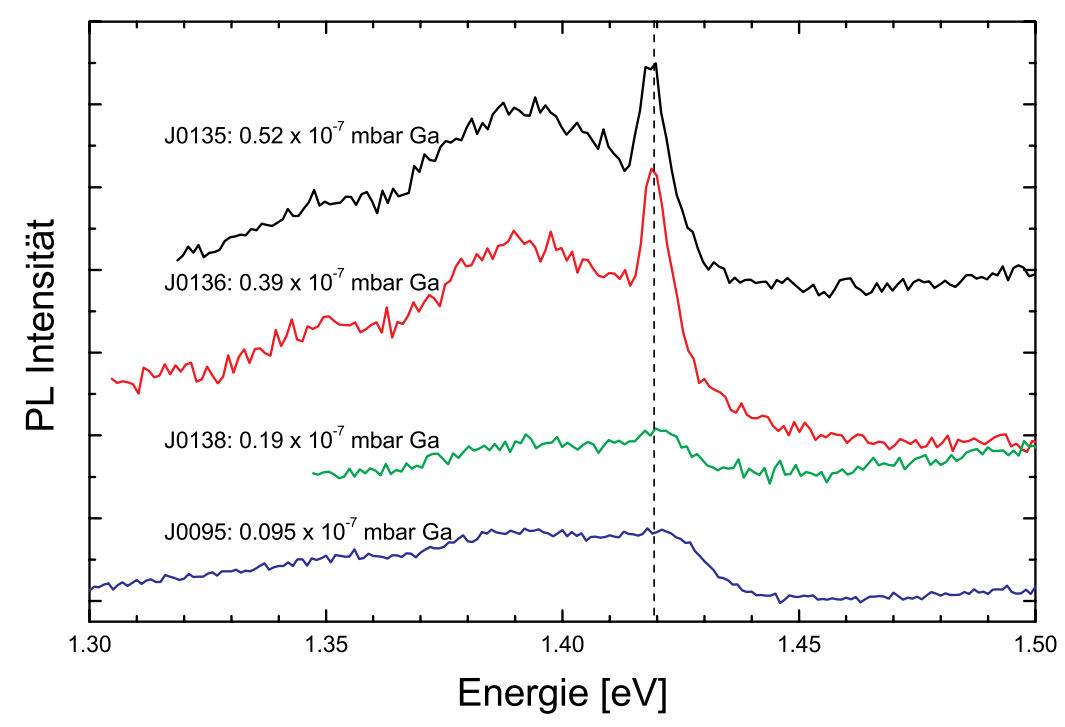

Abbildung 6.12: Verhalten der Lumineszenz bei $1.42 \mathrm{eV}$ bei Erhöhung des Ga-Angebots. Nahe der Stöchiometrie (Proben J0135 und J0136) ist eine scharfe ZPL sichtbar, im Stickstoffreichen nicht. Die Spektren sind vertikal zueinander verschoben.

unterschiedlichem Ga-Fluss gewachsen wurden. Probe J0135 wurde dabei genau bei stö- 
chiometrischen Bedingungen gewachsen, die anderen bei entsprechend stickstoffreicheren Bedingungen. Aus den strukturellen Charakterisierungen (siehe Kapitel 5) ist bereits bekannt, dass in den Proben J0135 und J0136 Ausscheidungen von $\mathrm{GaMn}_{3} \mathrm{~N}$ vorhanden sind, gleichzeitig liegt die Mn-Konzentration noch unter der EDX-Nachweisgrenze von 0.5\%. Es ist klar zu sehen, dass trotzdem noch $\mathrm{Mn}$ in der GaN-Matrix in verdünnter Form eingebaut wird, da eine klare Lumineszenz bei $1.42 \mathrm{eV}$ sichtbar ist mit ausgeprägter ZPL. Die Proben J0138 und J0095 sind im Wachtumsfenster für GaMnN und daher ohne Ausscheidungen und mit Mn-Konzentrationen im Prozent-Bereich. Trotz dieser unterschiedlichen Wachstumsbedingungen ist die Lumineszenz bei $1.42 \mathrm{eV}$ in allen Proben detektierbar. Es gibt also keine Korrelation zwischen dem Auftreten einer zweiten Phase und dem Verschwinden der Lumineszenz, wie es in Abbildung 6.11 angedeutet wurde. Im Gegenteil scheint die optische Qualität der Proben J0135 und J0136 trotz Ausscheidungen deutlich besser zu sein, als im extrem Stickstoffreichen, wie die scharfe ZPL zeigt. Ausscheidungen in kleiner Konzentration beeinträchtigen die Photolumineszenz also nicht, erst bei hohen Konzentrationen werden durch die vielen Grenzflächen nicht-radiative Zentren in hoher Konzentration kreiert.

Es lässt sich allerdings eine Korrelation finden zwischen dem Vorhandensein der bandkantennahen Lumineszenz und der Mn-Lumineszenz. Wenn im UV-Bereich von etwa $3.0-3.5 \mathrm{eV}$ keine Lumineszenz detektierbar war, so fehlte auch die infrarote Mn-Linie, bei deutlichen Lumineszenzen im UV war in allen Mn-dotierten Proben auch die $1.42 \mathrm{eV}$ Linie sichtbar. Dies deutet auf einen Zusammenhang der (exzitonischen) Lumineszenzen nahe der Bandkante und dem intra-3d-Übergang von Mn hin.

Ein weiterer Zusammenhang ergibt sich zwischen der Kristallqualität und der MnLumineszenz. Die Proben mit hoher Qualität zeigten eine relativ scharfe ZPL mit Halbwertsbreiten von $10 \pm 2 \mathrm{meV}$, je nach Probe. Bei niedriger Kristallqualität war diese scharfe Linie nicht erkennbar und komplett mit dem breiten Phononenseitenband verschmolzen.

\subsubsection{Verbesserung durch Saphir-Substrat}

Zur Verbesserung der Materialqualität wurden GaMnN-Schichten auf Saphir-Substrat gewachsen, auf dem bereits eine GaN-Schicht durch MOCVD aufgebracht wurde. Schon in Kapitel 5.2.3 wurde mittels Röntgenbeugung die deutlich erhöhte Kristallqualität der GaMnN-Schichten nachgewiesen. In den PL-Messungen nahe der Bandkante (siehe Kapitel 6.1) zeigen sich ebenfalls wesentliche Verbesserungen in der optischen Qualität der Proben. Diese höhere Qualität hat auch Auswirkungen auf die intra-3d-Lumineszenz von Mn.

\subsubsection{Aufspaltung und Verspannungsabhängigkeit der ZPL}

In Abbildung 6.13 ist die Mn-Lumineszenz zweier Proben, die bei ähnlichen Wachstumsbedingungen auf unterschiedlichen Substraten gewachsen worden sind, im direkten Vergleich zu sehen. Die scharfe Linie bei $1.30 \mathrm{eV}$ ist ein intra-3d-Übergang von $\mathrm{Fe}^{3+}$ [132]. Diese Linie ist in den MOCVD-gewachsenen GaN-Pufferschichten schon vor dem Wachstum der GaMnN-Schicht detektierbar und auf eine Fe-Verunreinigung in eben dieser Pufferschicht 
zurückzuführen. Da sie mit gelben Licht aus der YL direkt angeregt werden kann und die GaN-Schichten für dieses Licht und die IR-Lumineszenz praktisch durchsichtig sind, ist diese Linie auch bei dicken Proben $(\approx 1 \mu \mathrm{m})$ sichtbar, obwohl die Eindringtiefe des HeCd-Lasers unterhalb $100 \mathrm{~nm}$ liegt.

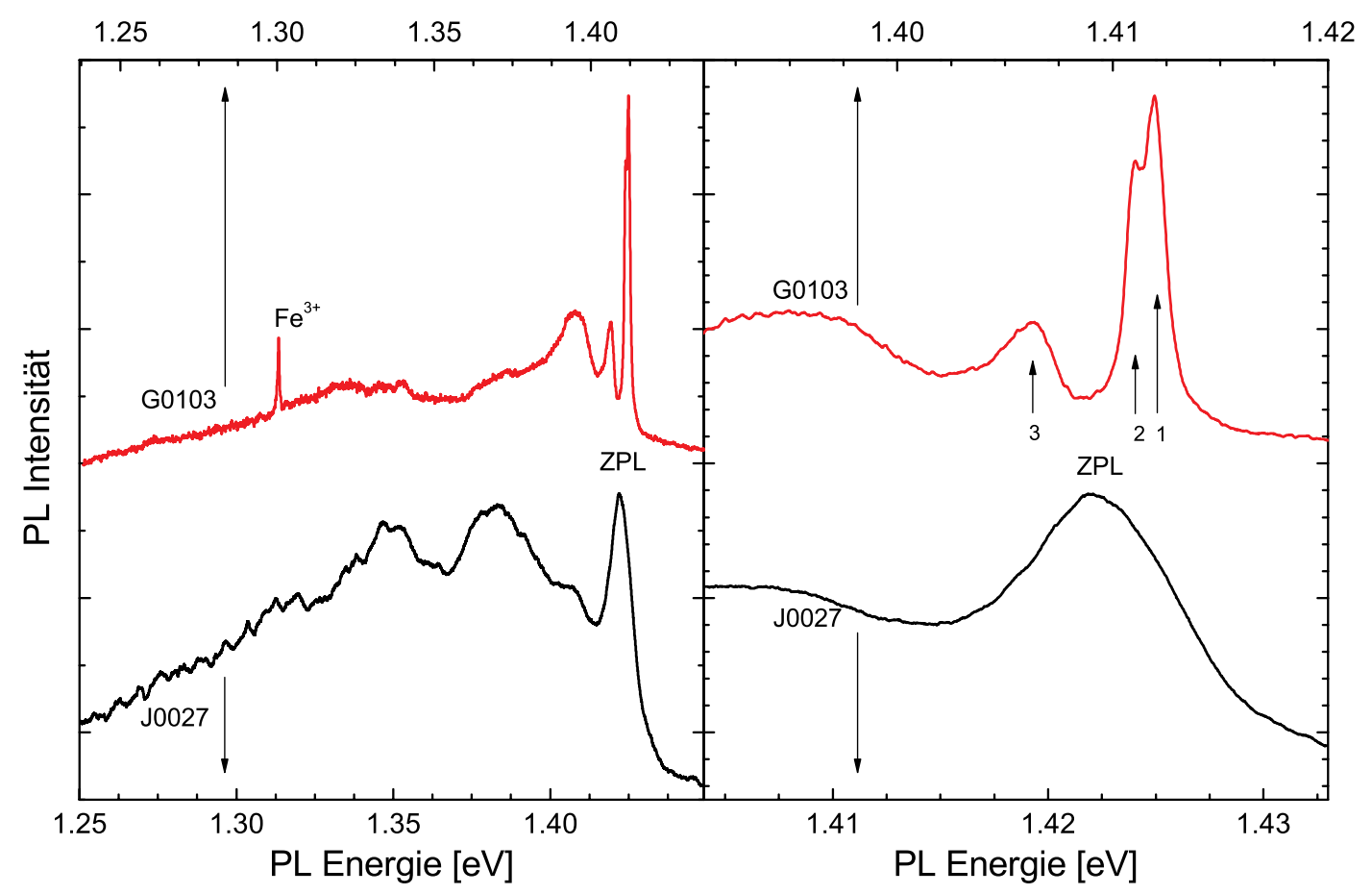

Abbildung 6.13: Vergleich von zwei Proben bei hoher Wachstumstemperatur auf Si-Substrat (J0027) und Saphir-Substrat (G0103). Die hochqualitative Saphir-Probe zeigt ein schwächeres Seitenband und eine Aufspaltung der ZPL.

Die auf Saphir gewachsene Probe G0103 zeigt eine deutlich stärkere ZPL im Vergleich zum phononischen Seitenband. Außerdem ist erkennbar, dass die ZPL in Wirklichkeit aus mindestens drei diskreten Linien zusammengesetzt ist, die rechts in Abbildung 6.13 vergrößert dargestellt sind. Auf Grund der großen Linienbreite konnte diese Aufspaltung in den Proben auf Si-Substrat nicht nachgewiesen werden. Schließlich ist die gesamte Mn-Lumineszenz um etwa $10 \mathrm{meV}$ zu niedrigeren Energien verschoben. Die genauen Parameter einer Anpassung mittels Gauß-Funktionen sind Tabelle 6.1 zusammengefasst. Die Serie G0103 bis G0131 ist bei ansonsten identischen Wachstumsbedingungen mit unterschiedlichem Mn-Angebot gewachsen worden, während J0027 als Vergleich bei ähnlichen Bedingungen auf Si-Substrat gewachsen wurde.

Im Vergleich wird deutlich, dass die Mn-Konzentration im Bereich $0.003-0.5 \%$ praktisch keinen Einfluss auf die Linienbreite hat, die nahezu konstant bleibt. Im statistischen Mittel ergibt sich für die breite ZPL 3 ein Wert von $3.32 \pm 0.20 \mathrm{meV}$, für ZPL 2 $0.83 \pm 0.03 \mathrm{meV}$ und für ZPL $11.04 \pm 0.02 \mathrm{meV}$. Dies ist schmaler als sämtliche in der 


\begin{tabular}{|c|c|c|c|c|c|c|c|}
\hline \multirow{2}{*}{$\begin{array}{l}\text { Probe } \\
\text { (Substrat) }\end{array}$} & \multirow{2}{*}{$\begin{array}{c}\text { Mn-Gehalt } \\
{[\%]}\end{array}$} & \multicolumn{3}{|c|}{ Position $[\mathrm{eV}]$} & \multicolumn{3}{|c|}{ FWHM [meV] } \\
\hline & & ZPL 1 & ZPL 2 & ZPL 3 & ZPL 1 & ZPL 2 & ZPL 3 \\
\hline J0027 (Si) & $\leq 0.5$ & \multicolumn{3}{|c|}{1.4228} & \multicolumn{3}{|c|}{8.8} \\
\hline G0103 (Saphir) & 0.5 & 5 & 1.41094 & 1.4 & 1.07 & 0.82 & 2.88 \\
\hline G0117 (Saphir) & 0.07 & 1.41269 & 1.41165 & 1.4 & 1.09 & 0.92 & 3.79 \\
\hline G0119 (Sal & 0.015 & 1.41218 & 1.41118 & 1.40 & 0.99 & 0.79 & 3.14 \\
\hline G0131 (Saphir) & 0.003 & 1.41265 & 1.41164 & 1.40626 & 1.02 & 0.77 & 3.47 \\
\hline
\end{tabular}

Tabelle 6.1: Aufgelöste Linien in Abbildung 6.13. Die verschiedenen Proben zeigen nur eine Verschiebung der Linien, die relativen Positionen sowie die Linienbreite ist nahezu konstant.

Literatur dokumentierten Absorptionsmessungen und zeigt die außergewöhnliche Qualität der Göttinger Schichten. Der Abstand von ZPL 1 und 2 beträgt 1.01 $\pm 0.01 \mathrm{meV}$ und zeigt im Rahmen der Messgenauigkeit keine Abhängigkeit von den Wachstumsparametern. Der Vergleich mit Probe J0027 auf einem anderen Substrat zeigt die empfindliche Abhängigkeit der Linienposition von diesem Verspannungseffekten. Zur genaueren Analyse dieses Effektes wurden die erhaltenen Daten daher mit den exzitonischen Linienpositionen der jeweiligen Proben korreliert.

Nach [90, 145] ist die Verspannung einer GaN-Probe direkt proportional zu einer Verschiebung der exzitonischen Lumineszenzen. Davydov et al. [85] bestimmten für das DBX eine Proportionalitätskonstante von $K_{P L}=20 \mathrm{meV} / \mathrm{GPa}$. Daher wurden in Abbildung 6.14 einige exzitonische und die intra-3d-Linien in Abhängigkeit von der Position der DBXLinie aufgetragen. Wie in Abbildung 6.14a) zu sehen, verschieben sich auch die anderen exzitonischen Linien entsprechend, diese Verschiebung kann durch eine lineare Funktion angepasst werden. In Abbildung 6.14b) ist dieselbe Anpassung für die drei ZPL der intra$3 d$-Lumineszenz von $\mathrm{Mn}^{3+}$ durchgeführt. Dies zeigt, dass eine Erhöhung der kompressiven Verspannung in der Basalebene zu einer Erniedrigung der Kristallfeldaufspaltung führt. ZPL 3 zeigt dabei eine Verschiebung, die signifikant verschieden von der von ZPL 1 und 2 ist. Außerdem ist diese Verschiebung deutlich kleiner. Dies zeigt, dass an dem Übergang der für ZPL 3 verantwortlich ist, andere Kristallfeldterme beteiligt sind als bei ZPL 1 und 2. Probe J0027 auf dem Si-Substrat zeigt nur eine auswertbare exzitonische Linie (DBX) und eine einzige breite ZPL. In Abbildung 6.14c) ist dieses Datenpaar zusammen mit den aus Abbildung 6.14b) erhaltenen Daten und Anpassungen aufgetragen. Man erkennt, dass die ZPL im Rahmen der Messgenauigkeit genau in der Mitte der vorhergesagten Position von ZPL 1 und 2 detektiert wird. Es ergibt sich damit für ZPL 1:

$$
E(\mathrm{ZPL} 1)=1.41592 \mathrm{eV}-(0.2953 \pm 0.0023)(E(\mathrm{DBX})-3.471 \mathrm{eV})
$$

bzw.

$$
E(\mathrm{ZPL} 1)=1.41592 \mathrm{eV}-(5.906 \pm 0.047) \mathrm{meV} / \mathrm{GPa} \cdot \sigma_{x x} .
$$

Eine unverspannte Probe mit einer DBX-Linie bei $3.471 \mathrm{eV}$ hätte also den ZPL 1Übergang bei $1.41592 \mathrm{eV}$. ZPL 2 zeigt genau das gleiche Verhalten mit einer um $1 \mathrm{meV}$ geringeren Energie. Für ZPL 3 erhält man:

$$
E(\mathrm{ZPL} 3)=1.40715 \mathrm{eV}-(0.085 \pm 0.017)(E(\mathrm{DBX})-3.471 \mathrm{eV})
$$




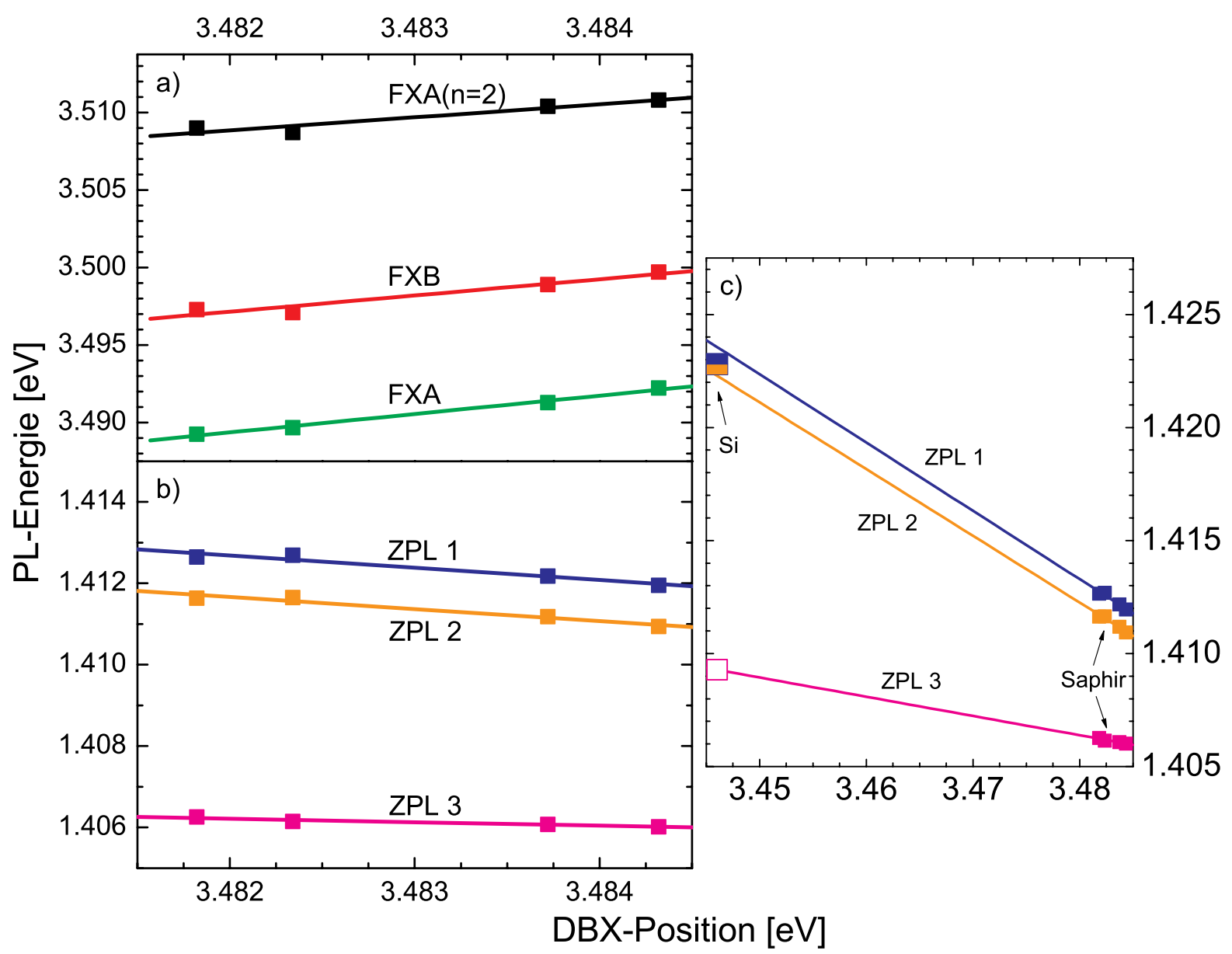

Abbildung 6.14: Verschiebung der a) exzitonischen Linien und b) intra-3d-Linien in Abhängigkeit von der DBX-Linienposition (die sich proportional zur Verspannung verschiebt) für die in Tabelle 6.1 analysierten Proben auf Saphir-Substrat. In c) ist Probe J0027 auf Si-Substrat zusätzlich eingetragen. Die durchgezogenen Linien sind eine lineare Anpassung der Daten aus a) bzw. b).

bzw.

$$
E(\mathrm{ZPL} 3)=1.40715 \mathrm{eV}-(1.70 \pm 0.34) \mathrm{meV} / \mathrm{GPa} \cdot \sigma_{x x}
$$

Diese Linie ist in Probe J0027 auf dem Si-Substrat nicht identifizierbar, die vorhergesagte Position ist in Abbildung 6.14c) mit einem leeren Symbol markiert. Vergleicht man diesen Wert von $1.4093 \mathrm{eV}$ mit dem Spektrum in Abbildung 6.10, so wird klar, dass die erste Schulter nach der ZPL nicht (oder nicht ausschließlich) aus einer Phononenreplik der $E_{2}^{L}$-Mode besteht, da sich exakt hier ZPL 3 befindet. Durch die tensile Verspannung ist der Abstand zu ZPL 1 und 2 so stark gewachsen, dass eine Überlagerung mit dem Seitenband auftritt.

Der von anderen Gruppen analysierte Übergang in Absorption variiert ebenfalls zwischen etwa 1.41 und $1.42 \mathrm{eV}$. Allerdings wurden keine exzitonischen Lumineszenzen oder andere Indikatoren für den Verspannungszustand berichtet. Lediglich bei den Volumenproben von Wolos et al. [35] kann von unverspannten Proben ausgegangen werden. Die dort berichtete Position der Absorptionslinie von $1.4166 \mathrm{eV}$ ist in guter Übereinstimmung 
mit dem entsprechenden Wert in Gleichung 6.1.

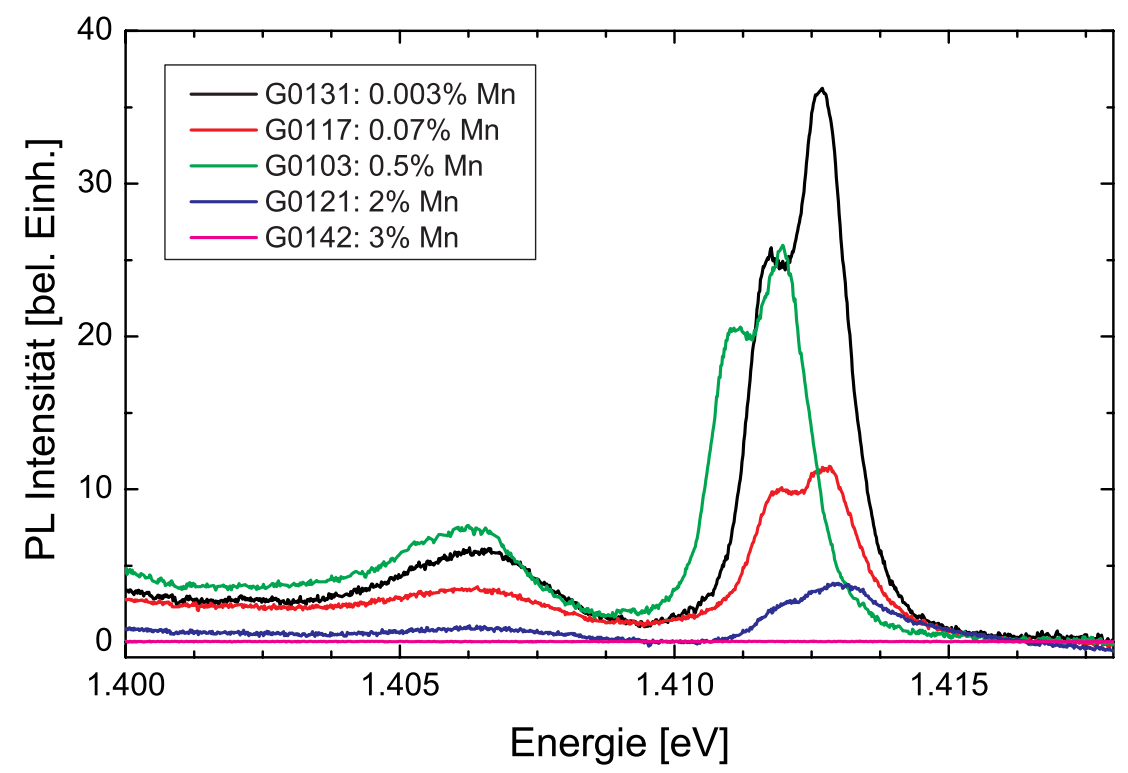

Abbildung 6.15: PL-Spektren im IR von hochqualitativem GaMnN mit verschiedenem Mn-Gehalt. Tendenziell wird die Lumineszenz mit steigendem Mn-Gehalt schwächer und breiter, bis sie komplett verschwindet.

In Abbildung 6.15 ist der intra-3d-Übergang verschieden stark dotierter Proben direkt miteinander verglichen. Diese Messungen wurden in einem Durchlauf durchgeführt, um maximale Vergleichbarkeit zu gewährleisten. Man erkennt, dass die Intensitäten dieses Übergangs stark schwanken, tendenziell aber bei niedrigen Konzentrationen höher liegen. Es gibt also keine Proportionalität zur Mn-Konzentration in den Proben. Bei Probe G0121 lässt sich außerdem eine Verbreiterung feststellen, ZPL 1 und 2 sind hier nicht mehr trennbar. In der hochdotierten Probe G0142 schließlich ist keinerlei IR-Lumineszenz detektierbar.

Zum Vergleich sei hier an Abbildung 6.6 erinnert, wo die exzitonischen Lumineszenzen in Abhängigkeit von der Mn-Konzentration analysiert wurden. Die dort vorgenommene Einteilung trifft exakt auch auf den IR-Bereich zu: Schwache und verbreiterte ExzitonLumineszenz korreliert mit einem Verschwinden des intra-3d-Übergangs in den hochqualitativen Proben. Eine weitere wichtige Korrelation ergibt sich mit den magnetischen Messungen. Probe G0142 zeigt ein ferromagnetisches Verhalten, mit einer Hysterese, die noch bei Raumtemperatur Bestand hat. Die unterhalb ein Prozent dotierten Proben zeigten keinerlei Ferromagnetismus. Die „Grenzprobe“ G0121 mit einem verbreiterten (und sehr schwachen) intra-3d-Übergang liefert eine mögliche Erklärung für diesen Phasenübergang im GaMnN-System. Die scharfen atomaren Niveaus, die in der PL detektiert werden, verbreitern sich bei höherer Konzentration und daraus folgender Wechselwirkung zwischen den Mn-Atomen. Schließlich bilden sich Störstellenbänder heraus, die nicht mehr optisch aktiv sind (zumindest nicht mit diesem scharfen Übergang), aber genau die ferromagnetischen Wechselwirkungen nach dem Doppelaustausch zulassen, weshalb dann der Ferromagnetismus einsetzt. Eine alternative Erklärung für das Verschwinden der Lumines- 
zenz könnte allerdings auch in der sich verschlechternden Kristallqualität liegen, die eine höhere Konzentration nicht-radiativer Defekte verursacht. Wie auch an Hand der exzitonischen Lumineszenz sichtbar, wird jegliche Lumineszenz bei höheren Mn-Konzentrationen unterdrückt, was schließlich auch den intra-3d-Übergang betrifft.

\subsubsection{Temperatur- und Leistungsabhängigkeit}

Auch an hochqualitativen Proben auf Saphir-Substrat wurden temperaturabhängige Messungen durchgeführt. Diese wurden wie in Kapitel 6.2.1.1 beschrieben durchgeführt und ausgewertet. Dabei war eine Auflösung von ZPL 1 und 2 auf Grund der apparativen Gegebenheiten nicht möglich, wie in Abbildung 6.16 sichtbar. ZPL 3 ist allerdings klar aufgelöst und kann daher analysiert werden. Im Einschub ist die Intensität des Gesamtsignals ZPL 1 und 2 ins Verhältnis zur Intensität von ZPL 3 gesetzt und gegen die Temperatur aufgetragen. Im Rahmen der Messgenauigkeit bleibt dieses Verhältnis im analysierbaren Temperaturbereich konstant, wie eine Anpassung zeigt.

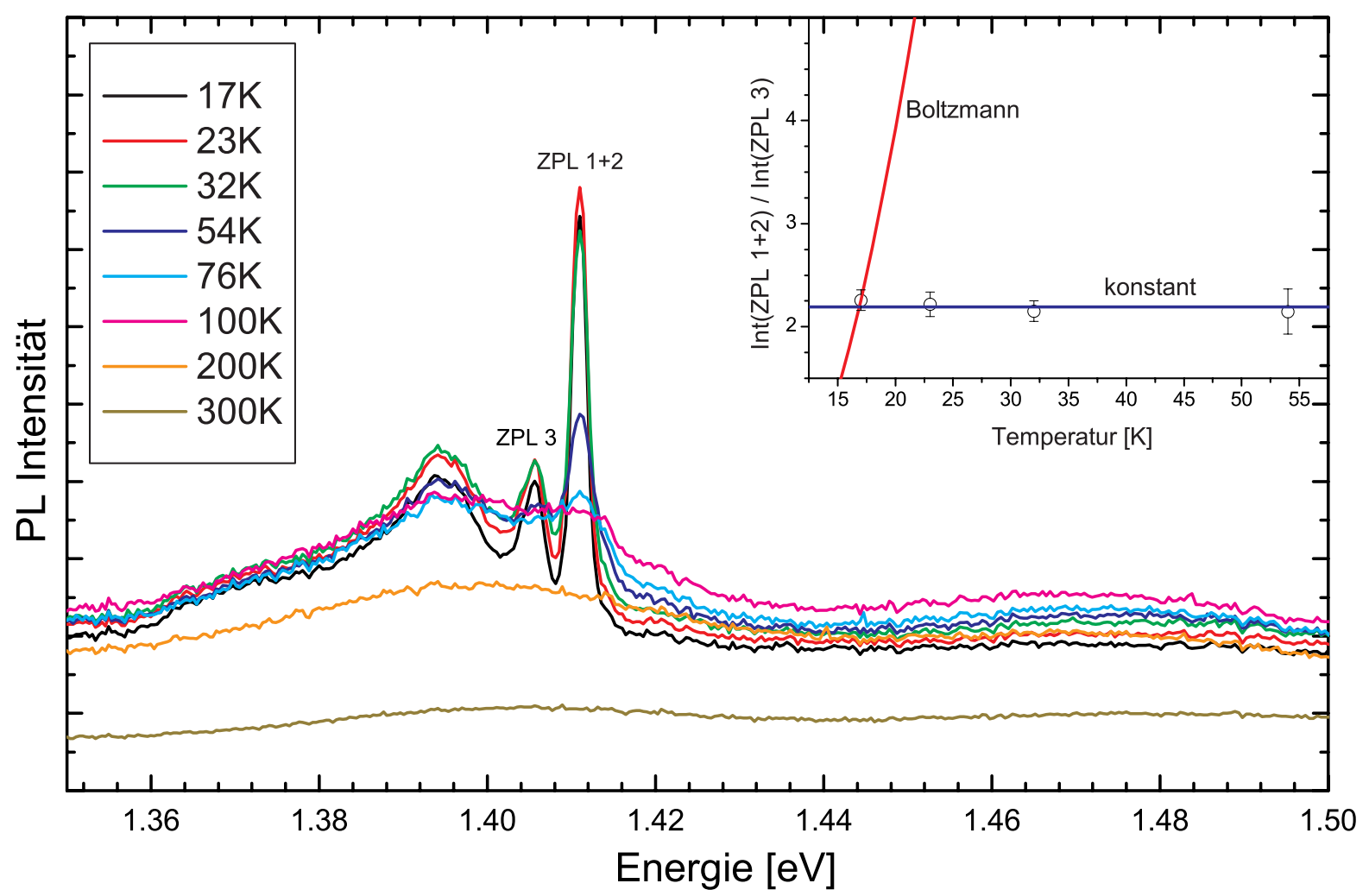

Abbildung 6.16: Temperaturabhängigkeit der Lumineszenz bei $1.4 \mathrm{eV}$ von Probe G0103. ZPL 1 und 2 sind nicht voneinander trennbar. Der Einschub zeigt das Verhältnis der Intensitäten von ZPL 1 und 2 zu ZPL 3 in Abhängigkeit von der Temperatur. In blau ist ein konstantes Verhältnis angenommen, in rot eine Abhängigkeit $\propto e^{\frac{-\Delta E}{k_{B} T}}$ mit der Energiedifferenz $\Delta E$ der betrachteten Linien.

Geht man davon aus, dass die verschiedenen ZPL einen einzigen intra-3d-Übergang als gemeinsamen Ursprung haben, so ergeben sich zwei verschiedene Möglichkeiten. Entweder ist der angeregte Zustand aufgespalten oder der Grundzustand. Im ersten Fall würde sich 
nach der Anregung eine thermische Population der Niveaus $i$ und $j$ ergeben nach dem Boltzmann-Gesetz $N_{i} / N_{j} \propto e^{\frac{-\left(E_{i}-E_{j}\right)}{k_{B} T}}$. Diese Temperaturabhängigkeit sollte sich auch im Verhältnis der Intensitäten widerspiegeln. Im zweiten Fall ändert sich die Besetzung mit der Temperatur nicht und man erwartet daher ein temperaturunabhängiges Intensitätsverhältnis. Damit wird aus Abbildung 6.16 klar, dass der Grundzustand ${ }^{5} T_{2}$ des $\mathrm{Mn}^{3+}{ }_{-}$Ions in mindestens zwei Zustände aufgespalten ist.

Leistungsabhängige Messungen zeigten im Rahmen der Messgenauigkeit keine Abhängigkeit der Lumineszenz von der eingestrahlten Laserleistung. Diese wurde von 0.15 bis $45 \mathrm{~mW}$ variiert, ohne dass sich Änderungen der Linienform ergaben. Die Intensität der Lumineszenz skalierte linear mit der Anregungsleistung. Das Fehlen einer Sättigung zeigt eine relativ hohe Konzentration des zu Grunde liegenden Defektes (hier: Mn-Atome) an. Da auch in den Proben mit dem niedrigsten Mn-Gehalt noch Konzentrationen oberhalb $10^{18} \mathrm{~cm}^{-3}$ gemessen wurden, ist diese Eigenschaft klar erfüllt.

\subsubsection{Anregungsmechanismus}

Um den Anregungsmechanismus des $\mathrm{Mn}^{3+}$-Übergangs ${ }^{5} E \rightarrow{ }^{5} T_{2}$ zu studieren, wurden Experimente mit anderen Lichtquellen zur Anregung durchgeführt. Dabei zeigte sich, dass weder mit den Linien eines Ar-Lasers im grünen und blauen Spektralbereich noch mit einer Halogenlampe über den gesamten sichtbaren und IR-Bereich eine Anregung möglich war. Diese Aussage muss allerdings insofern eingeschränkt werden, als dass mit der Halogenlampe nur wesentlich niedrigere Lichtleistungen als mit Laserquellen realisiert werden können. Eine Abschätzung ergab eine etwa eine Größenordnung schwächere Anregung als mit dem HeCd-Laser bei $325 \mathrm{~nm}$. Da dieser um etwa 2.5 Größenordnungen abgeschwächt werden kann, bis die Lumineszenz unter ähnlichen Bedingungen nicht mehr nachweisbar ist, bedeutet dies, dass eine Anregung unterhalb der Bandlückenenergie in jedem Fall mindestens eine Größenordnung schwächer ist als oberhalb. Bei den Hauptlinien des Ar-Laser (514 und $488 \mathrm{~nm}$ ) standen erheblich höhere Leistungen zur Verfügung. Die direkte Anregung der intra-3d-Lumineszenz ist hier um mindestens vier Größenordnungen schwächer.

Die bereits in Kapitel 6.2.2 angesprochene Korrelation des Auftretens der MnLumineszenz mit dem Auftreten von bandkantennahen exzitonischen Lumineszenzen gibt einen weiteren Hinweis auf einen möglichen Anregungsmechanismus. Nach der Anregung durch den HeCd-Laser sind in jeder Probe Elektronen und Löcher vorhanden, aber nur in manchen sind die Lebensdauern lang genug um Exzitonen zu bilden, die wiederum lang genug leben um strahlend rekombinieren zu können. Es ist daher anzunehmen, dass die Anregung des intra-3d-Übergangs durch ein Exziton in der Schicht verursacht wird. Dies ist durch einen so genannten Auger-artigen Prozess möglich, wie er in Abbildung 6.17 dargestellt wird.

\section{Zusammenfassung}

In diesem Kapitel wurde gezeigt, dass in GaMnN ein intra-3d-Übergang von $\mathrm{Mn}^{3+}$ detektiert werden kann. Nur in Proben mit relativ hoher Kristallqualität tritt eine scharfe Null-Phononen-Linie (ZPL) bei $1.41-1.42 \mathrm{eV}$ auf, die ansonsten mit dem phononischen 


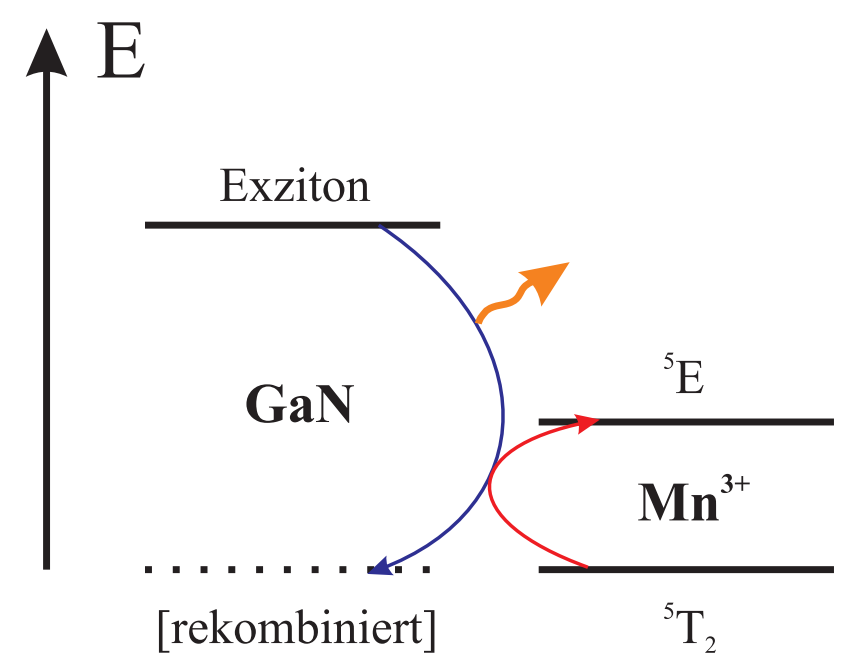

Abbildung 6.17: Auger-artiger Prozess zur Anregung von intra-atomarer Lumineszenz. Die bei der Rekombination eines Exzitons freiwerdende Energie wird teilweise genutzt, um das MnElektronensystem in einen angeregten Zustand anzuheben. Die restliche Energie wird beispielsweise photonisch oder phononisch abgegeben.

Seitenband verschmilzt. Bei den hochqualitativen Proben auf Saphir-Substrat sinkt die Linienbreite nahezu um den Faktor 10 und es wird eine Feinstruktur von drei verschiedenen ZPL sichtbar. Durch temperaturabhängige Messungen ließ sich nachweisen, dass der Grundzustand des Übergangs aufgespaltet ist und diese Aufspaltung durch Kristallfeldkomponenten mit der gleichen Symmetrie wie die biaxialen Verspannungen der epitaktischen Schichten hervorgerufen wird. Für die Anregung der Lumineszenz wird ein Auger-artiger Prozess vorgeschlagen. Es konnte festgestellt werden, dass in hochqualitativen, ferromagnetischen Proben dieser scharfe Übergang verschwindet, was auf eine Bandbildung nach dem Doppelaustausch hindeutet. 


\subsection{Magneto-optische Messungen}

Die Photolumineszenz von GaMnN wird von einem charakteristischen intra-3d-Übergang (siehe Kapitel 6.2) und im Falle von hochqualitativen Proben der exzitonischen Bandkantenlumineszenz (siehe Kapitel 6.1) bestimmt. Intra-3d-Lumineszenzen sind geeignet, genaue Aussagen über die lokale Umgebung des Übergangsmetalles zu machen, wie etwa der Kristallfeldaufspaltung und lokale Symmetrien. Die exzitonische Lumineszenz ist eng mit den Elektronen und Löchern in Leitungs- und Valenzband verknüpft, welche eine entscheidende Rolle für magnetische Kopplungen spielen können. Um auf diese detaillierten Informationen Zugriff zu bekommen, reichen normale PL-Messungen nicht aus. Es wird zusätzlich ein Magnetfeld benötigt, was zu charakteristischen Aufspaltungen der intra-3dLumineszenzen führt und die Ladungsträger in einem magnetischen Halbleiter beeinflusst, was sich in den Exziton-Spektren niederschlagen kann.

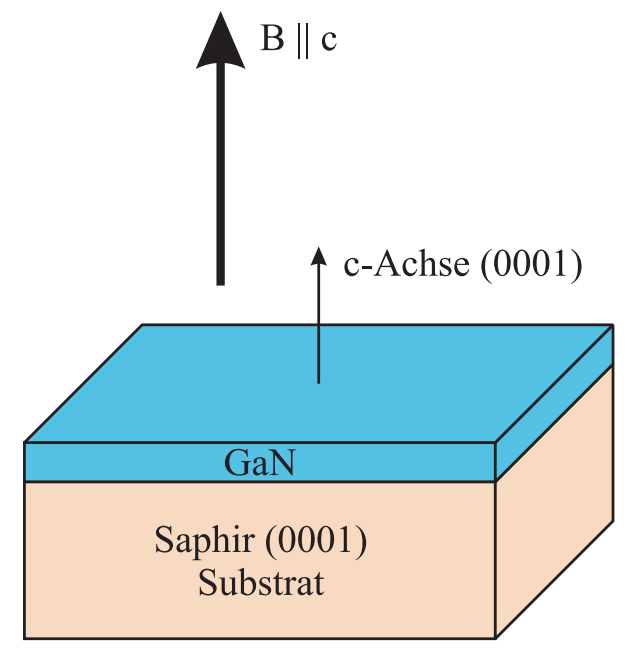

Faraday-Geometrie

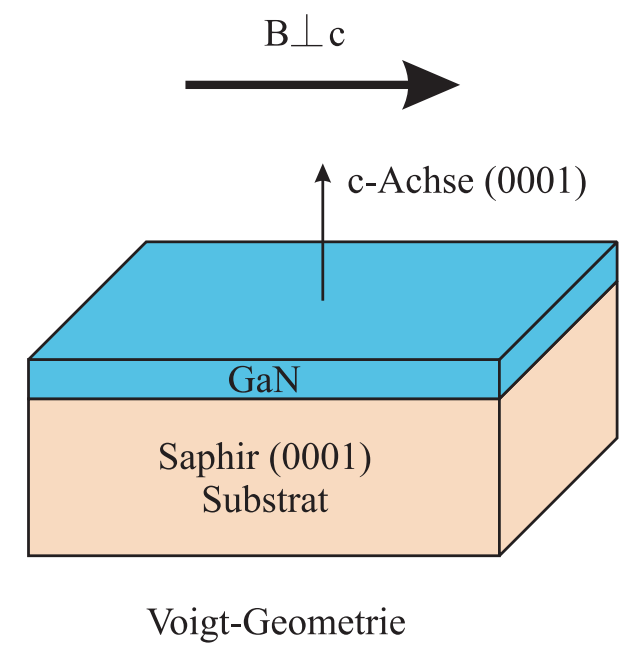

Voigt-Geometrie

Abbildung 6.18: Faraday- und Voigt-Geometrie in der Magneto-Optik.

Die magneto-optischen Experimente wurden im Rahmen einer Zusammenarbeit an der Universität Grenoble durchgeführt. Die Anregung erfolgt mittels eines HeCd-Lasers, die Proben sind in einem Verdampfer-Kryostaten montiert, in dem durch supraleitende Spulen ein Magnetfeld von bis zu 11 T erzeugt werden kann. Das Magnetfeld ist dabei parallel zur Probennormale gerichtet, in der so genannten Faraday-Geometrie (siehe Abbildung 6.18). Das Licht wird in einem $0.5 \mathrm{~m}$-Spektrometer mit einem 600er Gitter spektral zerlegt und mit einer CCD-Kamera detektiert. Details des Aufbaus sind beispielsweise in [146] nachlesbar.

\subsubsection{Nahe der Bandkante}

In Abbildung 6.19 sind die PL-Spektren für verschiedene Magnetfeldstärken dargestellt. Probe G0103 mit einem Mn-Gehalt von etwa 0.5\% zeigt dabei ein Verhalten, wie es 
auch von reinem GaN bekannt ist. Die exzitonischen Signale zeigen dabei keine deutliche Abhängigkeit vom Magnetfeld. In Probe G0121 mit einem deutlich höheren Mn-Gehalt ist bereits aus Abbildung 6.19 eine Verschiebung der FXA-Linie zu höheren Energien mit steigendem Magnetfeld sichtbar.

a) G0103: $0.5 \% \mathrm{Mn}$

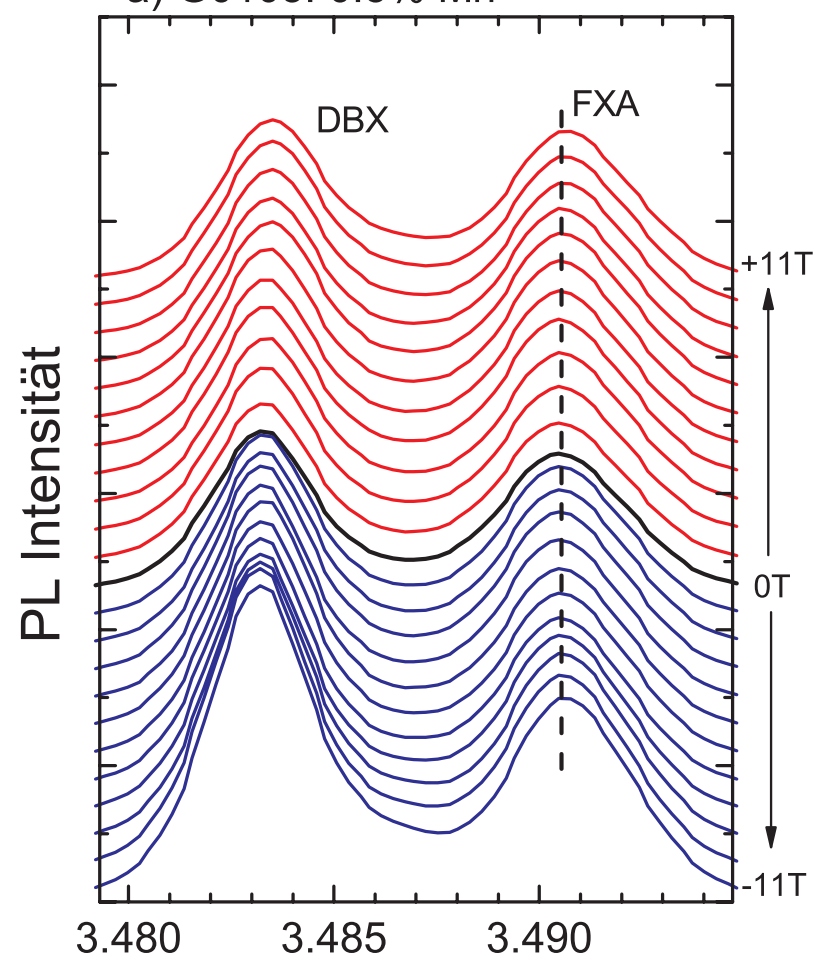

b) G0121: $2 \% \mathrm{Mn}$

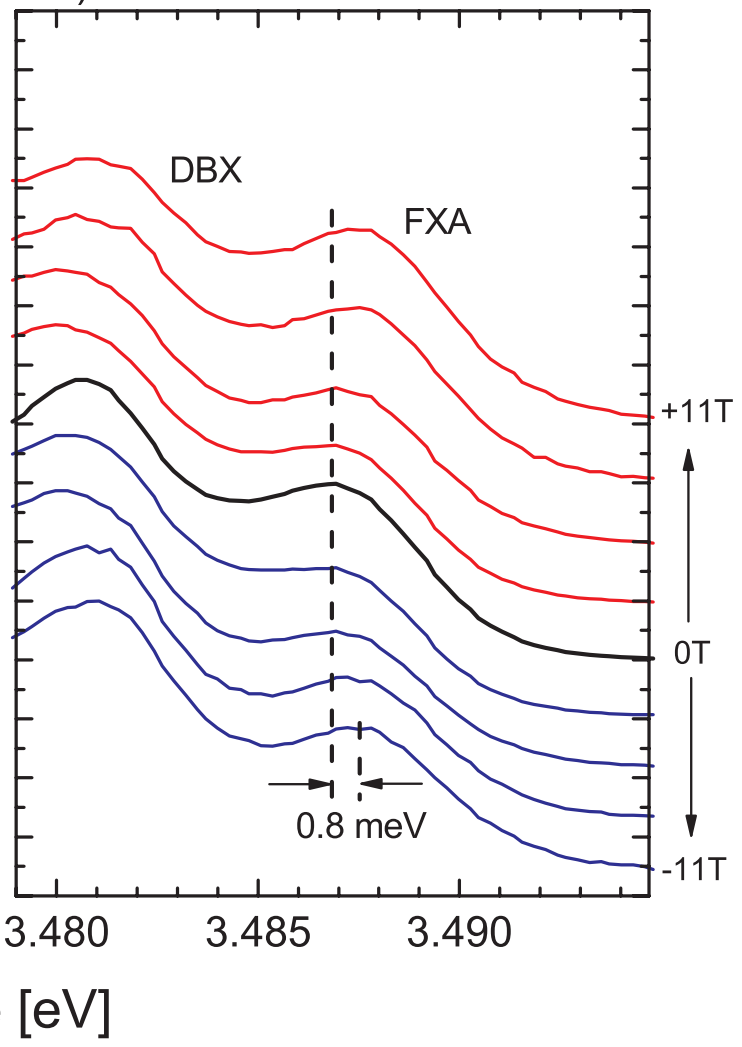

Abbildung 6.19: PL-Spektren nahe der Bandkante für zwei Proben mit unterschiedlichen Mn-Gehalt in Abhängigkeit vom Magnetfeld. Das FXA zeigt in der hochdotierten Probe eine Verschiebung zu höheren Energien.

Um dieses Verhalten genauer zu analysieren, wurden die Lagen der exzitonischen Signale mittels einer Anpassung exakt bestimmt. Dabei wurden die FXA- und FXB-Linien, sowie der angeregte Zustand $\mathrm{FXA}(\mathrm{n}=2)$ berücksichtigt. Auf Grund seiner Lage als schwache Schulter der FXA-Linie konnten die Ergebnisse der FXB-Lumineszenz nicht weiter analysiert werden, die beiden FXA-Linien sind in Abbildung 6.20 zusammengefasst.

Das Verhalten von Exzitonen in einem Magnetfeld wird von zwei Faktoren bestimmt. Zum Einen gibt es einen Zeeman-Term direkt proportional zum B-Feld, der aus der Spinaufspaltung von Leitungs- und Valenzband folgt. Er hängt direkt mit den effektiven gFaktoren der beteiligten Elektronen und Löcher zusammen. Zum Anderen gibt es die diamagnetische Verschiebung, die mit der Erniedrigung der Exziton-Bindungsenergie durch ein externes Magnetfeld zusammenhängt und quadratisch vom B-Feld abhängt. Insgesamt erwartet man also: 

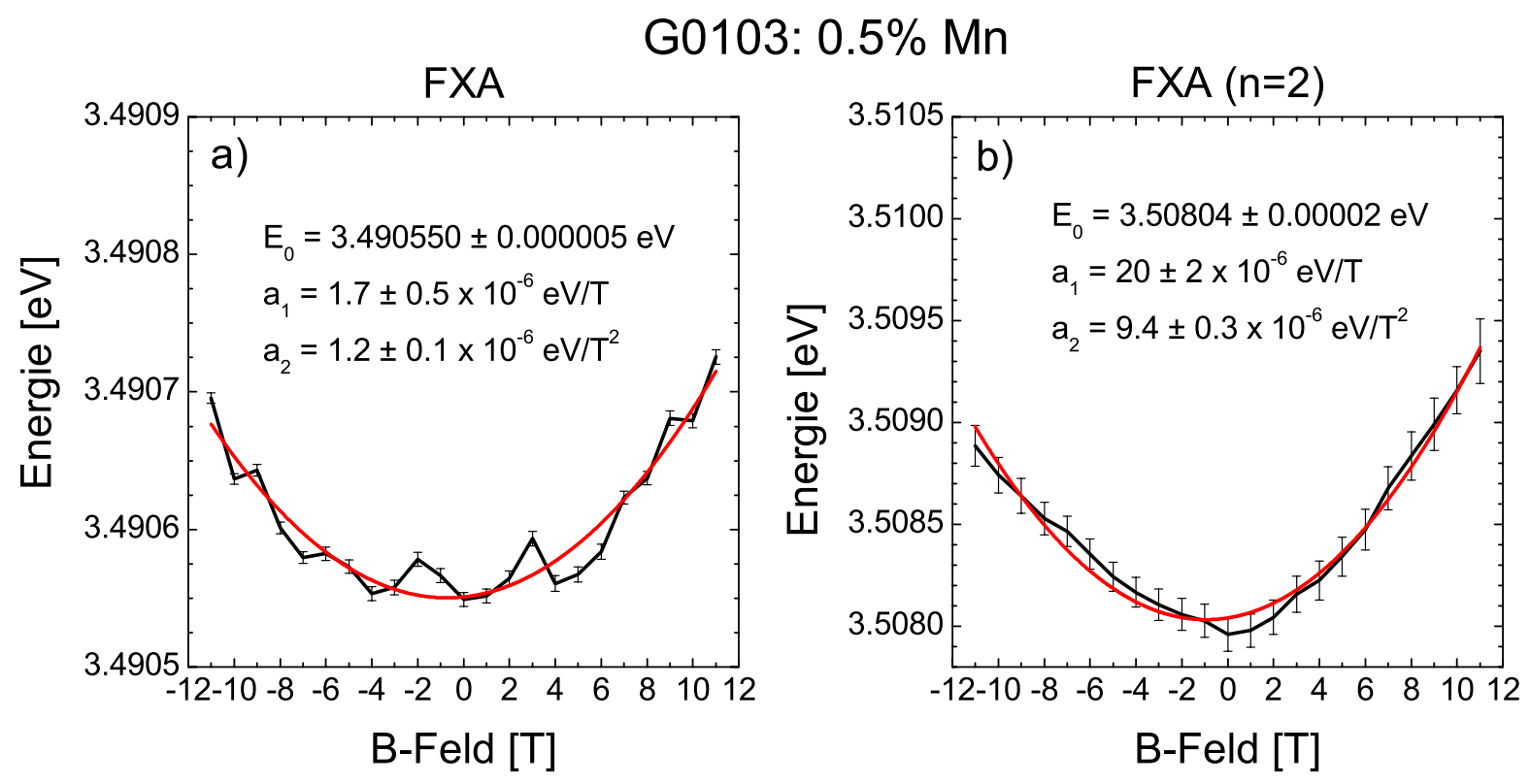

G0121: $2 \% \mathrm{Mn}$
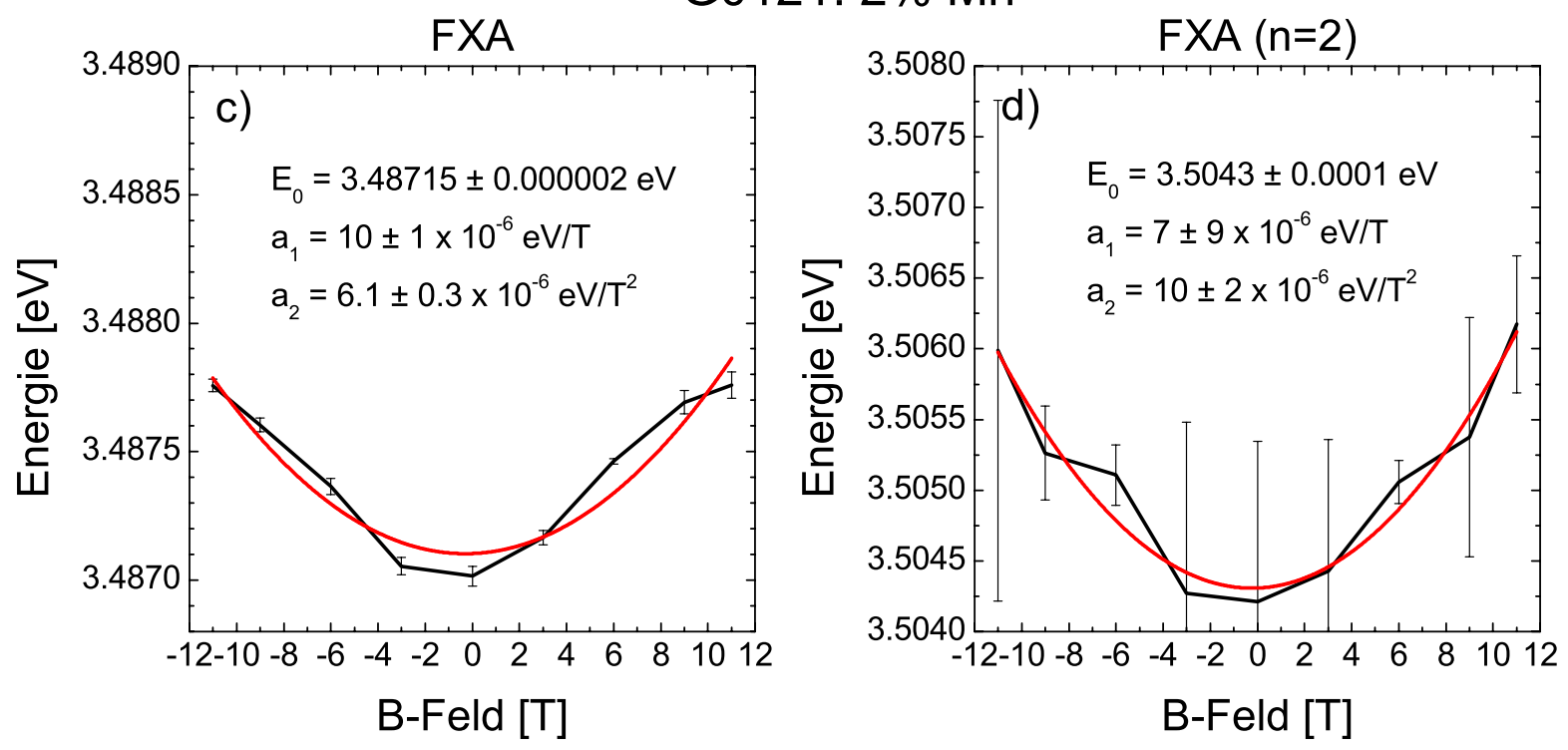

Abbildung 6.20: Position der Signale von FXA und FXA $(\mathrm{n}=2)$ in Abhängigkeit vom Magnetfeld. Die Daten (schwarz) wurden mit einer Parabel, $E(B)=E_{0}+a_{1} B+a_{2} B^{2}$, angepasst (rot).

$$
E(B)=E_{0}+a_{1} B+a_{2} B^{2}
$$

Dabei beschreibt $a_{1}$ den Zeeman-Term und $a_{2}$ die diamagnetische Verschiebung. Wie aus Abbildung 6.20a) ersichtlich, kann FXA in der niedrig-dotierten Probe sehr gut mit dieser Abhängigkeit beschrieben werden. Der vernachlässigbar kleine Zeeman-Term deutet auf ungefähr gleich große g-Faktoren für Elektron und Loch hin, in Übereinstimmung mit der Literatur $[147,129,148]$ für reines GaN. Auch die diamagnetische Verschiebung von $1.2 \mu \mathrm{eV} / T^{2}$ ist in relativ guter Übereinstimmung mit den Angaben von $1.84 \mu \mathrm{eV} / T^{2}[147]$ 
und $2.3 \mu \mathrm{eV} / T^{2}$ [149]. Der 2s-Zustand von FXA ist in Abbildung 6.20b) analysiert. Er zeigt eine etwa um den Faktor 10 erhöhte ähnlich geartete Abhängigkeit vom Magnetfeld. Vergleichbares wurde auch schon von Shields et al. beobachtet [148], dort allerdings in Reflexion bei dem 2p-Zustand. Insgesamt zeigt die niedrig dotierte GaMnN-Probe G0103 also ein Verhalten wie es auch von undotiertem GaN zu erwarten ist, das Mn hat also keinen messbaren Einfluss auf die magneto-optischen Eigenschaften.

Die hoch dotierte Probe G0121 ist in Abbildung 6.20c) und d) analysiert. Auf Grund der etwas breiteren und schwächeren Signale ist die Datenqualität hier nicht so hoch wie in der niedrig dotierten Probe, aber es lassen sich trotzdem ein paar qualitative Aussagen machen. Die FXA-Linie zeigt keine perfekte Parabel, es ist aber offensichtlich, dass sowohl der Zeeman-Term als auch die diamagnetische Verschiebung deutlich gegenüber der niedrig dotierten Probe erhöht sind. Dies war auch schon aus Abbildung 6.19 ersichtlich. Die Ursache der Erhöhung der diamagnetischen Verschiebung ist noch unklar. Eine Erniedrigung der Exziton-Bindungsenergie in GaMnN, die diesen Effekt erklären könnte, kann ausgeschlossen werden, da die Energiedifferenz zwischen FXA und FXA(n=2) (entsprechend 3/4 der Exziton-Bindungsenergie) unverändert bleibt. Eine weiterführende Analyse ist nicht Ziel dieser Arbeit und wird an dieser Stelle nicht weiter verfolgt.

Die Erhöhung des Zeeman-Terms kann mit dem so genannten „giant Zeeman effect“ erklärt werden. Dabei wechselwirken die GaN-Bänder mit den lokalen Spins der Mn-Atome und zeigen als Folge dieser Wechselwirkung eine erhöhte Spin-Aufspaltung. Dies wurde in Kapitel 2.3.1 bereits diskutiert, im Falle von GaMnN handelt es sich um den $s, p-d-$ Austausch. Ein entsprechendes Modell wurde von Pacuski et al. angewandt, um magnetooptische Reflexions- und Absorptionsspektren von GaMnN zu beschreiben [150]. Dabei wurde $N_{0}(\alpha-\beta)$ zu $1.2 \pm 0.2 \mathrm{eV}$ bestimmt, was bei einer Annahme von $N_{0} \alpha=0.2 \mathrm{eV}$ (einem typischen Wert für DMS) auf eine starke ferromagnetische Kopplung zwischen den Mn-Atomen und den Valenzband-Löchern hindeutet. Der von Pacuski et al. gemessene Zeeman-Term hat dabei die Größenordnung $a_{1} \approx 360 \mu \mathrm{eV} / T$ in einer Probe mit $0.5 \%$ Mn. Da der „giant Zeeman effect" linear mit der Mn-Konzentration skaliert, wären bei einer Konzentration von 2\% dann Zeeman-Verschiebungen von über $1 \mathrm{meV} / \mathrm{T}$ zu erwarten. Probe G0121 zeigt allerdings nur $a_{1}=10 \pm 1 \mu \mathrm{eV} / T$. Damit ist die beobachtete Mn-Loch-Kopplung nur extrem schwach ausgeprägt. Einerseits kann dies damit erklärt werden, dass nur ein Bruchteil der Mn-Atome elektrisch aktiv sind. Andererseits halten sich bei der PL-Emission möglicherweise die freien Exzitonen nur in Bereichen auf, wo die Mn-Konzentration geringer ist, während sie in den Bereichen hoher Konzentration durch die Mn-Atome oder angelagerte Defekte gebunden werden. Eine spinodale Entmischung wurde an Hand von EDX-Karten von Tore Niermann bereits nachgewiesen [113]. Von theoretischer Seite betrachtet ist eine schwache $p$ - $d$-Kopplung durchaus zu erwarten, da die Mn- $d$-Zustände relativ tief in der Bandlücke liegen im Vergleich zu beispielsweise GaMnAs.

Der angeregte Zustand $\mathrm{FXA}(\mathrm{n}=2)$ in $6.20 \mathrm{~d})$ zeigt keine signifikanten Änderungen mit der Mn-Konzentration, allerdings muss diese Aussage auf Grund der Datenqualität stark eingeschränkt werden. Die Fehlerbalken spiegeln nur die Standard-Abweichung aus der Anpassungsroutine wider. Da besonders bei hohen Feldern die Intensität dieser Linie stark abnahm, ist hier mit einem erhöhten systematischen Fehler dieser Routine zu rechnen. 
Die magneto-optischen Messungen zeigen also eine vorhandene, wenn auch schwach ausgeprägte, Kopplung der Mn-Atome mit den Löchern des Valenzbandes. Solch eine Kopplung zwischen magnetischer Verunreinigung und den freien Ladungsträgern der Matrix ist eine Grundvorraussetzung für die technische Nutzbarkeit der verdünnten magnetischen Halbleiter als Spininjektoren, die für den Fall von GaMnN damit gegeben zu sein scheint. Wie die Messungen von Pacuski et al. [150] mit deutlich stärkerem $p$ - $d$-Austausch zeigen, ist dabei noch eine Optimierung der vorhandenen Proben möglich.

\subsubsection{Bei $1.4 \mathrm{eV}$}

Von Marcet et al. wurden bereits ausführliche magneto-optische Studien am intra-3dÜbergang von $\mathrm{Mn}^{3+}$ in GaN durchgeführt [139, 37, 146]. Als Ergebnis wurde ein Modell mit einem dynamischen Jahn-Teller-Effekt gefunden, mit dem die experimentellen Daten in Übereinstimmung zu bringen waren. Dies wurde in Kapitel 2.2.2 dargelegt, Abbildung 2.15 dort zeigt das Resultat.

\subsubsection{Absorption}

Im Rahmen der Zusammenarbeit mit der Universität Grenoble wurden vergleichbare Messungen mit derselben Apparatur wie bei Marcet et al. auch an den in Göttingen hergestellten Proben unternommen. Die Transmissionsspektren wurden mittels einer in [146] beschriebenen Methode in Absorptionsspektren umgewandelt. In Abbildung 6.21 sind Absorptionsspektren ohne Magnetfeld für Proben mit unterschiedlicher Konzentration abgebildet. Der bekannte intra-3d-Übergang ${ }^{5} T_{2} \rightarrow{ }^{5} E$ von $\mathrm{Mn}^{3+}$ ist bei etwa $1.415 \mathrm{eV}$ in allen Proben sichtbar und skaliert mit der Mn-Konzentration, die mittels SIMS bestimmt wurde.

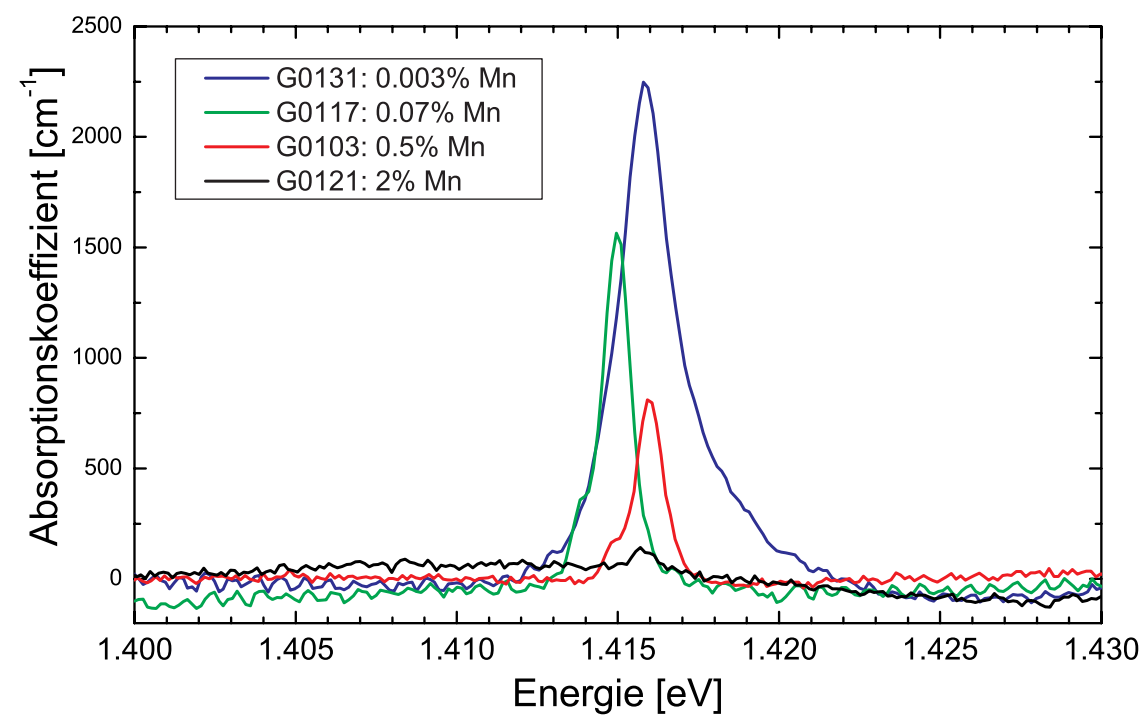

Abbildung 6.21: Absorptionsspektren von GaMnN mit unterschiedlicher Mn-Konzentration. 
In [37] wurde bereits ein linearer Zusammenhang zwischen der Fläche des Absorptionssignals und der Mn-Konzentration berichtet. Angewandt auf die gemessenen Proben ergibt sich die in Tabelle 6.2 zusammengefasste Gegenüberstellung.

\begin{tabular}{|c|c|c|}
\hline Probe & \multicolumn{2}{|c|}{ Mn-Gehalt [\%] } \\
& (SIMS) & (Absorption) \\
\hline G0121 & 2 & 0.3 \\
G0103 & 0.5 & 0.11 \\
G0117 & 0.07 & 0.05 \\
G0131 & 0.003 & 0.005 \\
\hline
\end{tabular}

Tabelle 6.2: Vergleich der Mn-Konzentration aus SIMS-Messungen mit der $\mathrm{Mn}^{3+}$-Konzentration aus Absorptionsmessungen.

Aus diesen Messungen lassen sich zwei wichtige Aussagen ableiten. Erstens ist das Mn in den vorliegenden Schichten tatsächlich im Ladungszustand 3+ vorhanden. Da Absorptionsmessungen bei tiefen Temperaturen ausschließlich vom Grundzustand des Systems ausgehen, kann im Gegensatz zur PL diese Aussage getroffen werden. Dies ist eine wichtige Erkenntnis im Hinblick auf magnetische Eigenschaften, da manche Theorien einen teilweise besetzten Zustand fordern, der im $\mathrm{Mn}^{3+}$ vorhanden ist, nicht aber im $\mathrm{Mn}^{2+}$ (siehe auch Kapitel 2.3.1). Außerdem zeigt es, dass sich das Fermi-Niveau tief in der Bandlücke befinden muss, da nur in diesem Fall $\mathrm{Mn}^{3+}$ in der GaN-Matrix stabil ist. Es ergibt sich auch ein weiterer Hinweis auf den Anregungsmechanismus der Photolumineszenz des gleichen Übergangs. Da sowohl PL als auch Absorption in den gleichen Proben parallel beobachtet werden konnten, ist es wahrscheinlich, dass der Anregungsprozess der PL vom $\mathrm{Mn}^{3+}$-Zustand ausgeht und nicht von einem $\mathrm{Mn}^{2+}$-Zustand, der während der Anregung ionisiert wird. Durch diese Koinzidenz ist demnach das Auftreten einer PL-Signatur bei $1.41 \mathrm{eV}$ ein Hinweis auf den $\mathrm{Mn}^{3+}$-Ladungszustand.

Die andere Aussage, die sich aus den Absorptionsmessungen gewinnen lässt, ist, dass die Zahl der detektierten $\mathrm{Mn}^{3+}$-Ionen bei höheren Konzentrationen erheblich von der Gesamtzahl der Mn-Atome (laut SIMS) abweicht. Dies kann mehrere Ursachen haben. Befindet sich beispielsweise ein Teil der Mn-Atome in Ausscheidungen, so ist dies mittels SIMS nicht von gelöstem Mn unterscheidbar. Tatsächlich zeigt Probe G0121 ein wenn auch extrem schwaches Signal der bekannten $\mathrm{GaMn}_{3} \mathrm{~N}-\mathrm{Ph}$ ase. Außerdem können sich die Mn-Atome in der GaN-Matrix häufen und Cluster bilden. In diesem Fall wird das Kristallfeld stark gestört und die beteiligten Atome tragen nicht zwangsläufig zum gleichen Übergang bei. Bei einer statistischen Verteilung der Mn-Atome auf Ga-Gitterplätzen wird dieser Effekt erst für Konzentrationen oberhalb 1\% messbar, die Konzentration $x$ müsste dann durch eine effektive Konzentration $x_{\text {eff }}=x(1-x)^{12}$ (es gibt 12 nächste Nachbarn im Ga-Untergitter) ersetzt werden. Dies kann die beobachteten Abweichungen also nur erklären, wenn eine sehr starke attraktive Wechselwirkung zwischen den Mn-Atomen angenommen wird, die diesen Effekt verstärkt. Mn auf anderen Gitterplätzen wird auf Grund der in Kapitel 5.3 dargelegten Ergebnisse ausgeschlossen. Ebenso wie andere MnAtome das Kristallfeld verändern, können aber auch intrinsische Defekte wie Leerstellen oder Versetzungen in der unmittelbaren Umgebung für den Effekt verantwortlich sein. 
Dies wäre in Übereinstimmung mit den strukturellen Messungen und anderen optischen Messungen, die bei hohen Mn-Konzentrationen eine schlechtere Qualität bestätigen. Die relativ gesehen schwächere Absorption von $\mathrm{Mn}^{3+}$ könnte theoretisch auch auf eine Änderung des Ladungszustands eines Teils der Mn-Ionen hindeuten. Allerdings müssten in diesem Fall eine extrem hohe Zahl an Donatoren oder Akzeptoren eingebaut werden, was unwahrscheinlich ist, da dieser Effekt bei niedrigen Mn-Konzentrationen nicht nachweisbar ist.
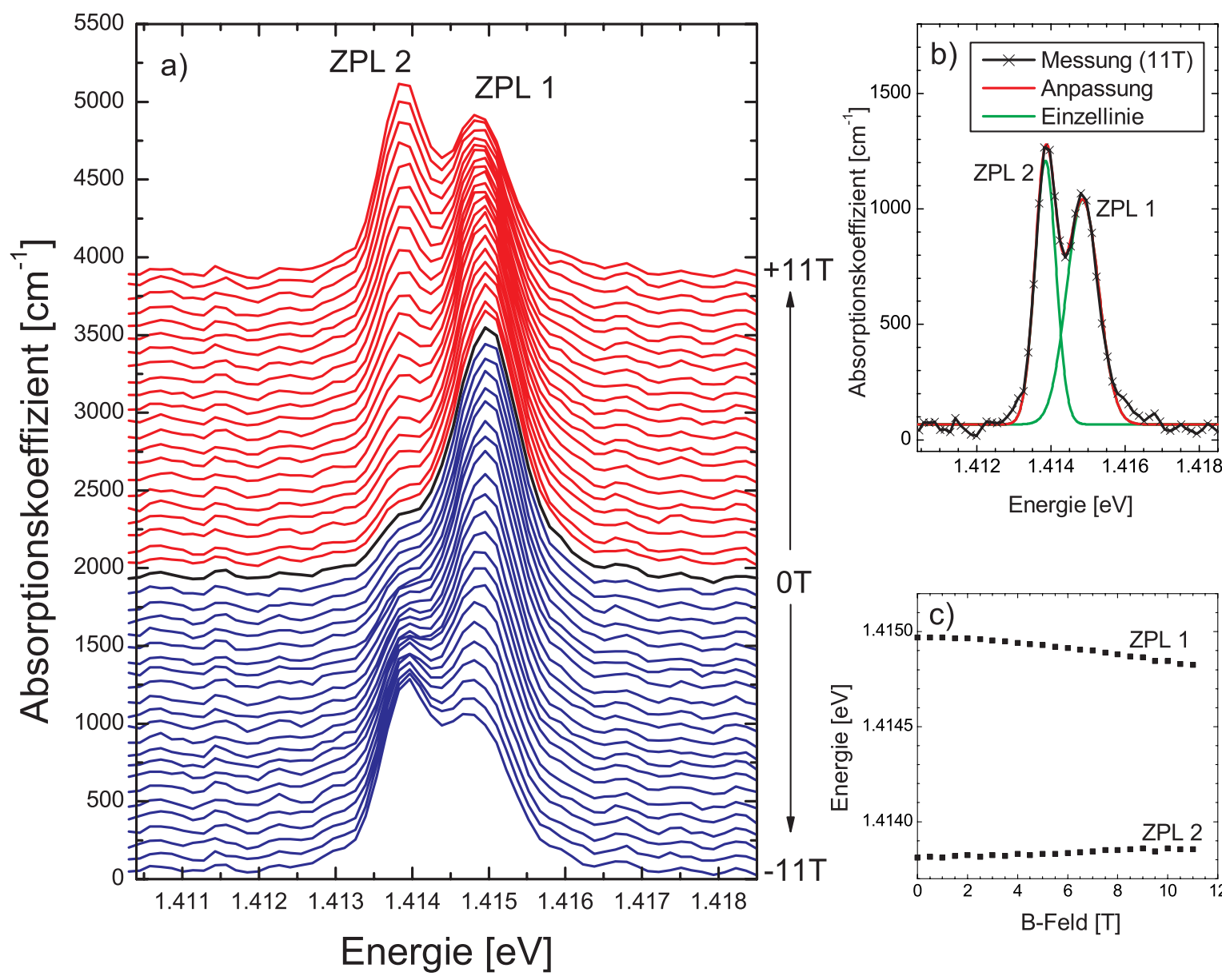

Abbildung 6.22: a) Absorptionsspektrum bei $1.4 \mathrm{eV}$ von Probe G0103 in Abhängigkeit vom Magnetfeld, aufgenommen bei $7 K$. b) Anpassung der Spektren mittels zweier Gauß-Funktionen. c) Verschiebung der Linienposition in Abhängigkeit vom Magnetfeld.

Abbildung 6.22a) zeigt das Absorptionsspektrum bei $1.4 \mathrm{eV}$ von Probe G0103 für $B=0 T, \pm 0.5 T, \pm 1 T, \ldots, \pm 11 T$ bei $7 \mathrm{~K}$. Es zeigt sich qualitativ das gleiche Verhalten wie bei den Proben von Marcet et al. . Ohne Feld wird das Spektrum von ZPL 1 dominiert, während bei höheren Feldern ZPL 2 stärker wird. Da Probe G0103 trotz einem optisch aktiven $\mathrm{Mn}^{3+}$-Gehalt von $0.11 \%$ etwas schmalere Linienbreiten aufweist als die besten Proben von Marcet et al. mit $0.03 \%$, können auch bei $7 K$ noch beide Linien durch eine Anpassung mittels zweier Gauß-Funktionen problemlos getrennt werden (siehe Ab- 
bildung 6.22b)). Es ergeben sich Linienbreiten von $1.0 \mathrm{meV}$ und $0.7 \mathrm{meV}$ für ZPL 1 und ZPL 2, unabhängig vom Magnetfeld. Die Lage der beiden Linien verändert sich mit dem Magnetfeld, wie in Abbildung 6.22c) dargestellt. Während ZPL 1 signifikant zu kleineren Energien schiebt, bleibt ZPL 2 nahezu unverändert, mit einer minimalen Verschiebung zu höheren Energien.

Die Verschiebung von ZPL 1 in Abhängigkeit vom Magnetfeld kann im Rahmen des Modells von Marcet et al. dadurch erklärt werden, dass ZPL 1 sich aus zwei nicht aufgelösten Linien zusammensetzt. Die Grundzustände der zugehörigen Übergänge (vergleiche L1 und L3 in Abbildung 2.15) zeigen unterschiedliche Abhängigkeiten vom Magnetfeld und schneiden sich bei etwa $2.5 \mathrm{~T}$, es findet also ein Intensitätstransfer zwischen diesen Übergängen statt. Bei niedrigem Feld dominiert der Anteil von L1, während bei hohem Feld L3 dominiert. Alle Vorhersagen des Modells bezüglich Abhängigkeit der Absorptionslinien von Temperatur und Magnetfeld lassen sich mit den im Rahmen dieser Arbeit analysierten Proben verifizieren. Vor allem die von Marcet et al. aufgezeigten Unstimmigkeiten [37] ihrer Messungen mit dem Modell von Wolos et al. [35] lassen sich durch die hochqualitativen Göttinger Proben reproduzieren. Dies ist also ein weiteres Zeichen für die Existenz eines dynamischen Jahn-Teller-Effekts in GaMnN im Gegensatz zu dem von Wolos et al. postulierten statischen Jahn-Teller-Effekt.

\subsubsection{Photolumineszenz}

Die Gruppen aus Warschau und Grenoble, die seit mehreren Jahren an den optischen Eigenschaften von GaMnN forschen und die entsprechenden Theorien zu magneto-optischen Untersuchungen entwickelt haben, konnten bisher in ihren Proben keine Photolumineszenz des entsprechenden intra-3d-Übergangs detektieren [151, 152]. Lediglich in einer hochverdünnten Probe konnte mittels CL Lumineszenz beobachtet werden [146]. Dies allerdings auch nur bei hohen Anregungsdichten in einem Raster-Elektronen-Mikroskop (SEM). Die Existenz des Übergangs ${ }^{5} E \rightarrow{ }^{5} T_{2}$ in PL-Spektren der Göttinger Proben ermöglicht dagegen magneto-optische Experimente auch in Lumineszenz, die bei einer Anregung mit einem Elektronenstrahl wie bei CL aus experimentellen Gründen praktisch unmöglich sind.

In Abbildung 6.23a) sind die Resultate der magnetfeldabhängigen PL-Messungen für die hochverdünnte Probe G0131 zu sehen. Während ohne Magnetfeld ZPL 1 etwa die doppelte Intensität wie ZPL 2 aufweist, sind die beiden Linien bei $11 T$ etwa gleich stark. Dies ist durch die vorhergesagte Magnetfeldabhängigkeit der angeregten Zustände der beteiligten Übergänge nach vollziehbar. Bei hohen Feldern ergibt sich eine Aufspaltung, die ZPL 2 als niedrigsten Zustand begünstigt, während bei niedrigen Feldern alle Niveaus des ${ }^{5} E$-Zustands nahezu entartet sind. Die gemessene Linienform erlaubt keine reproduzierbare Anpassung mittels Gauß-Funktionen, daher wurden Voigt-Funktionen benutzt, die eine Faltung aus Gauß- und Lorentz-förmigen Kurven darstellen. Es existiert damit ein weiterer freier Parameter pro Anpassung, wodurch eine gute Qualität der Anpassung erreicht wurde (siehe Abbildung 6.23b)). Die etwas unphysikalische Wahl dieser Funktion wird dadurch gerechtfertigt, dass hier nur die Position der Linien analysiert werden soll, welche auf diese Weise sehr gut bestimmt werden können. Die Position in Abhängigkeit 

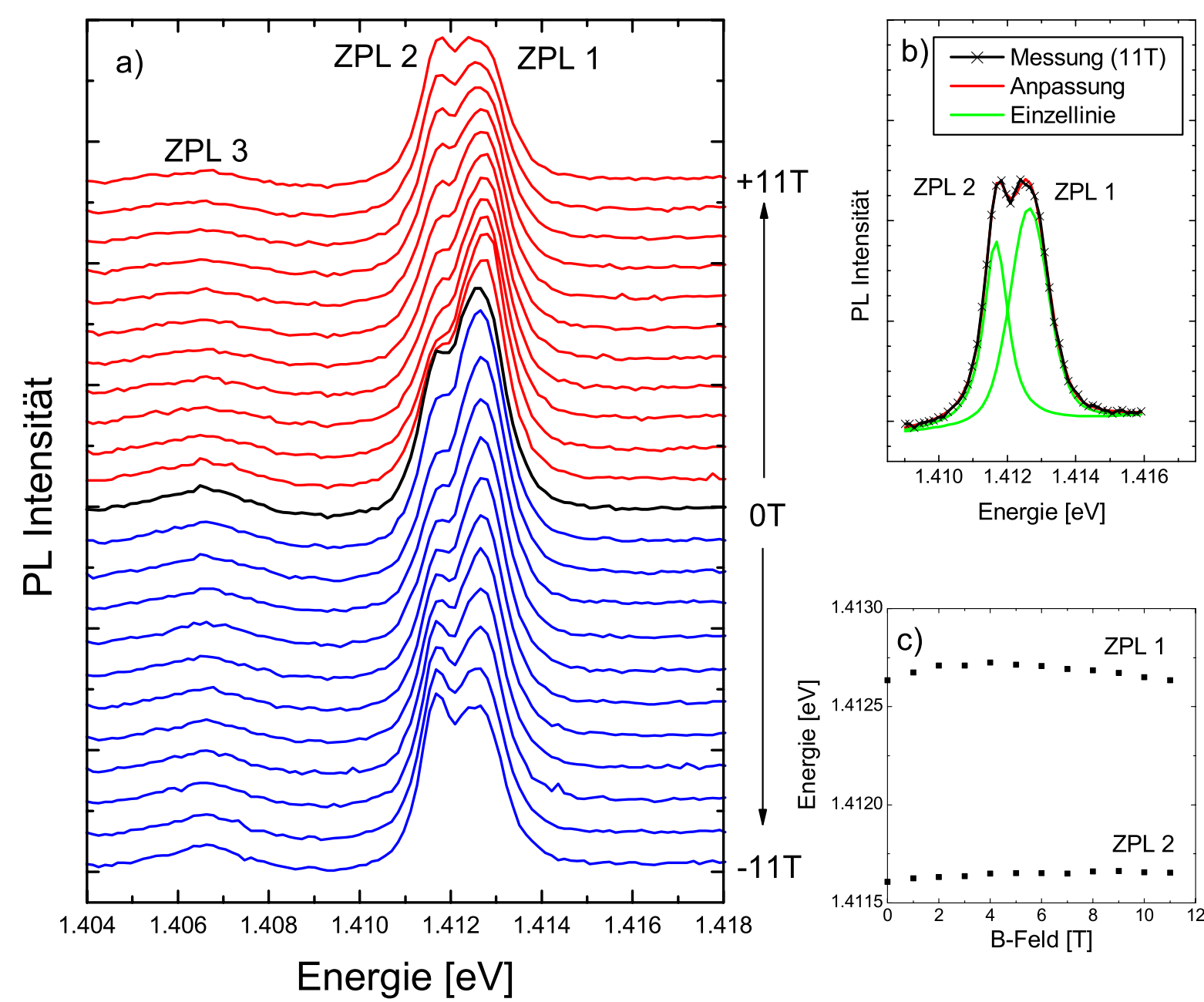

Abbildung 6.23: a) PL-Spektrum bei $1.4 \mathrm{eV}$ von Probe G0131 in Abhängigkeit vom Magnetfeld, aufgenommen bei $7 \mathrm{~K}$. b) Anpassung der Spektren mittels zweier Voigt-Funktionen. c) Verschiebung der Linienposition in Abhängigkeit vom Magnetfeld.

vom angelegten B-Feld ist in Abbildung 6.23c) zusammengefasst. Ähnlich wie in Absorption zeigt ZPL 2 nur eine minimale Erhöhung der Energie bei hohen Feldern. ZPL 1 allerdings verschiebt sich bei schwachen Feldern zu signifikant höheren Energien, um bei hohen Feldern wieder in die entgegengesetzte Richtung zu schieben.

Das Verhalten von ZPL 1 lässt sich durch zwei verschiedene Effekte erklären. Bei niedrigen Feldern spaltet der Endzustand des Übergangs sofort mit linearem Term auf, während der angeregte Anfangszustand mit einem quadratischen Term beginnt, bis er ab etwa $2 T$ ebenfalls linear wird und parallel zum Grundzustand verläuft. In diesem ersten Bereich wird daher die Übergangsenergie erhöht. Danach bewirkt die zunehmende Aufspaltung des Anfangszustands eine thermische Population der Unterniveaus, die eine Intensitätsverschiebung von L1 zu L3 bewirkt, analog zu den Ergebnissen der Absorptionsmessungen.

Weitere Informationen lassen sich durch temperaturabhängige Messungen bei hohen 


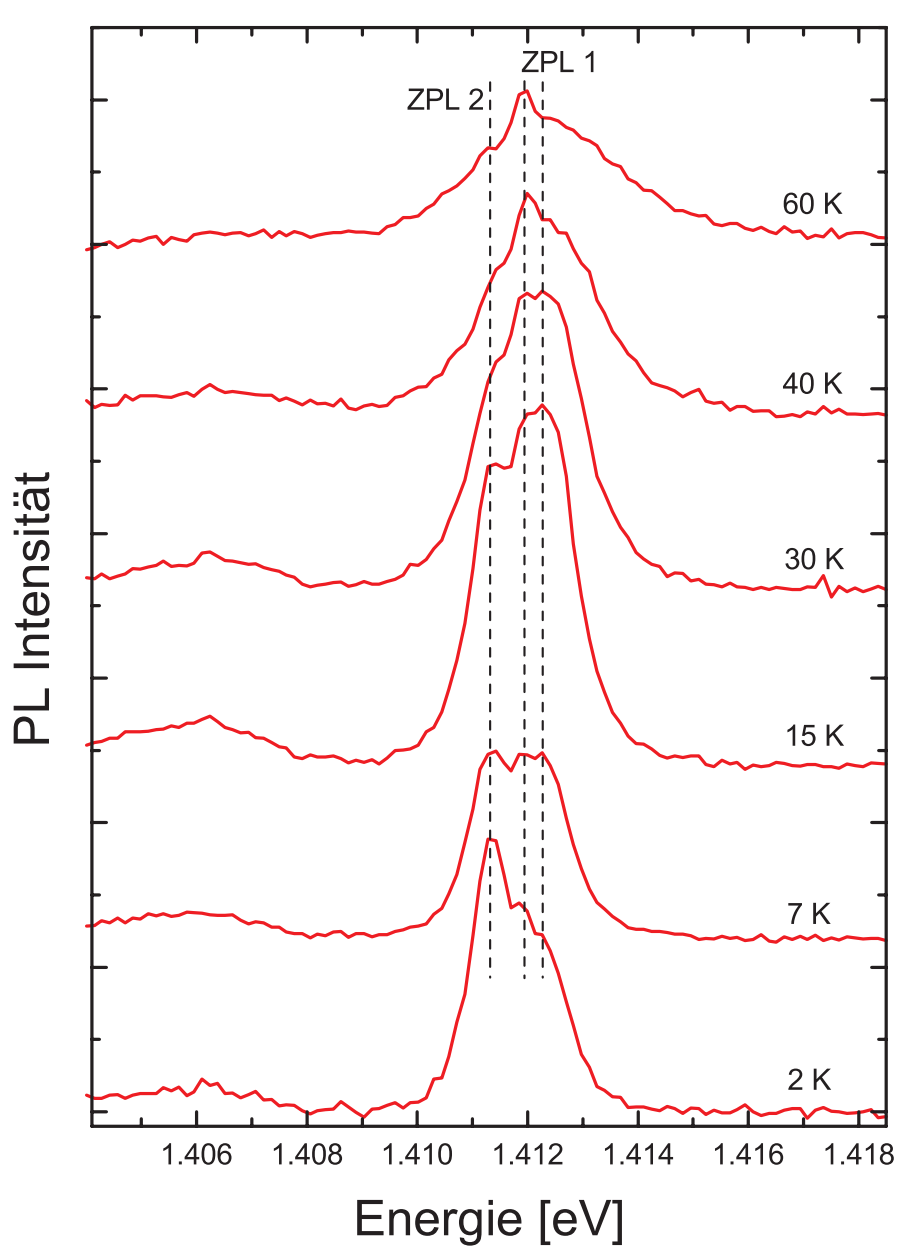

Abbildung 6.24: PL-Spektren bei $1.4 \mathrm{eV}$ von Probe G0131 in Abhängigkeit von der Temperatur bei einem B-Feld von 11 Tesla.

Feldern gewinnen, da die dann vorhandenen großen Aufspaltungen zu einem charakteristischen Verhalten auf Grund der thermischen Population führen. In Abbildung 6.24 sind entsprechende Messungen an Probe G0131 bei $11 \mathrm{~T}$ zu sehen. Während bei $2 K$ ZPL 2 dominiert und ZPL 1 nur als Schulter auftritt, ist die energetisch höher liegende ZPL 1 bei höheren Temperaturen stärker ausgeprägt. Zwar ist bei hohen Temperaturen auf Grund der Verbreiterung der Linien keine numerische Anpassung möglich, es ist aber aus den Spektren ersichtlich, dass ZPL 1 aus zwei Übergängen besteht, deren ungefähre Lage durch die Linien in Abbildung 6.24 angedeutet ist. ZPL 2 wird nur durch einen klar identifizierbaren Übergang verursacht.

Das Ergebnis der Messungen in Faraday-Geometrie ist in Abbildung 6.25 eingezeichnet. ZPL 1, 2 und 3 sind mit dem aus Absorptionsmessungen entwickelten Modell durch die eingezeichneten Übergänge erklärbar. ZPL 1 liegen dabei zwei verschiedene Übergänge zu Grunde, die sich aber nur um etwa $0.3 \mathrm{meV}$ unterscheiden und daher nur in temperaturabhängigen Messungen (Abbildung 6.24) schwach unterscheidbar waren.

Zusätzlich zu den bisher präsentierten Messungen in Faraday-Konfiguration wurden von 


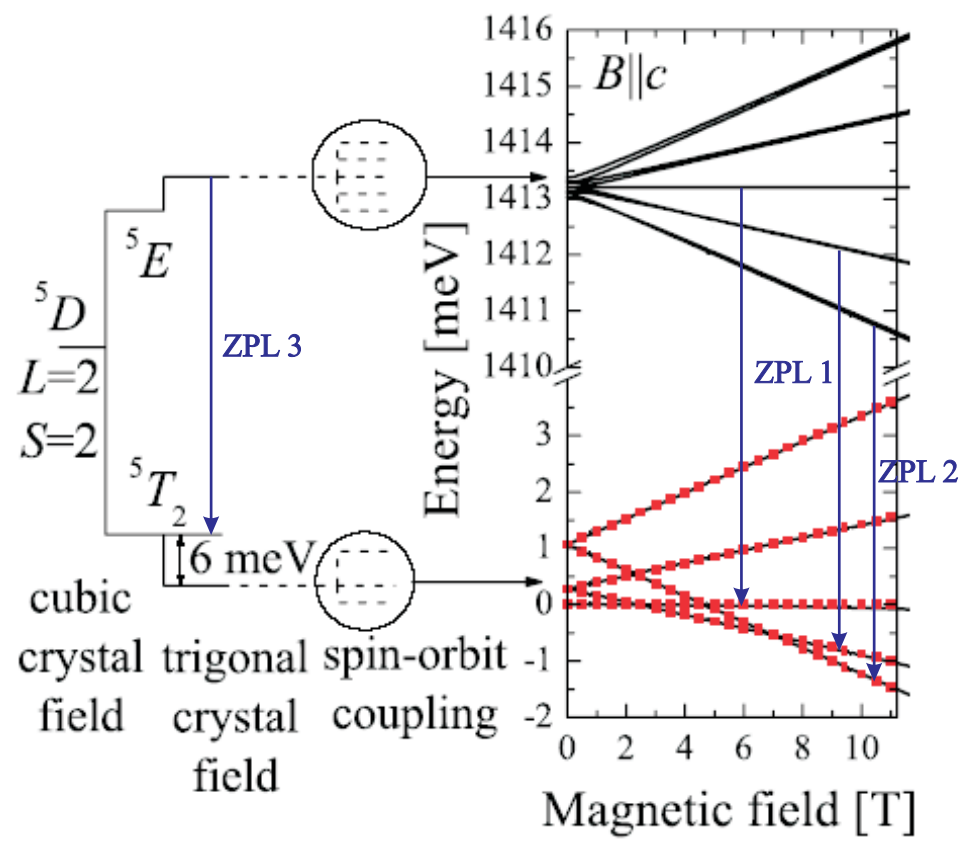

Abbildung 6.25: PL-Übergänge nach dem Modell von Marcet et al. (vgl. Abbildung 2.15).

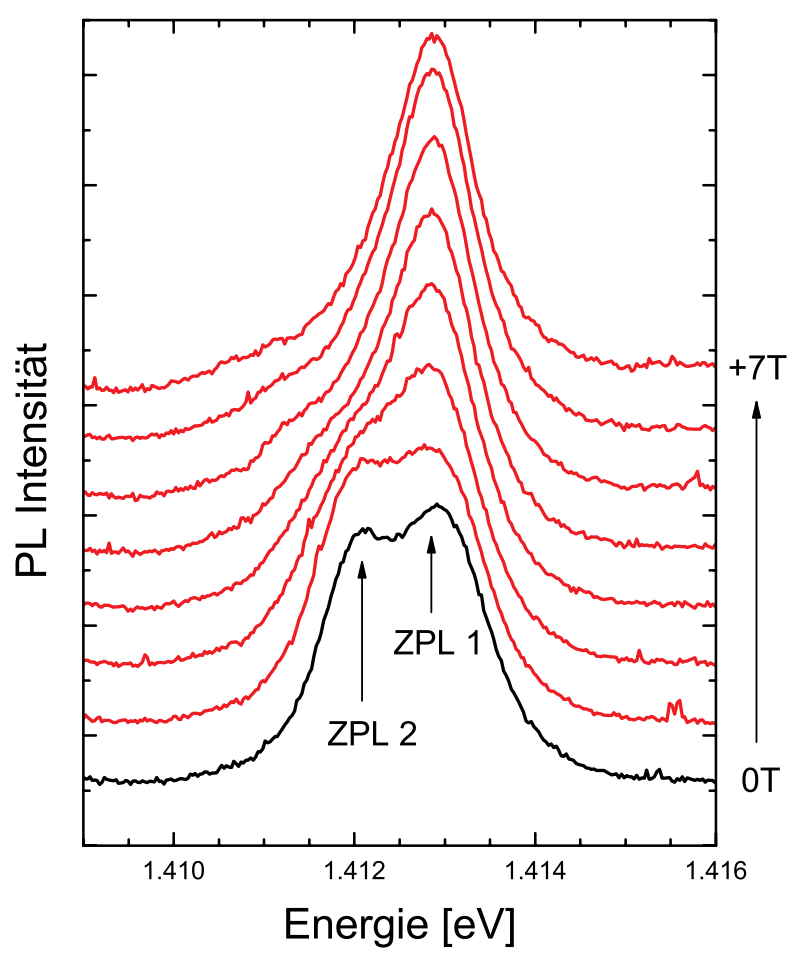

Abbildung 6.26: PL-Spektren bei $1.4 \mathrm{eV}$ von Probe G0117 in Abhängigkeit vom Magnetfeld in VoigtKonfiguration, gemessen bei $1.8 \mathrm{~K}$.

Wojciech Pacuski an Probe G0117 noch Messungen mit einem hochauflösenden System 
in Voigt-Konfiguration (siehe Abbildung 6.18) durchgeführt. Wie in Abbildung $6.26 \mathrm{zu}$ sehen, verschwindet ZPL 2 bei etwa $3 T$, oberhalb ist ausschließlich ZPL 1 detektierbar.

Auch dieses Ergebnis lässt sich mit dem von Marcet et al. entwickelten Modell erklären. In Voigt-Geometrie gehört ZPL $1 \mathrm{zu}$ einem Übergang aus dem niedrigsten ${ }^{5}$ E-Niveau, während ZPL 2 aus den höher liegenden Niveaus entsteht (siehe Abbildung 2.15 ganz rechts). Bei der niedrigen Temperatur von $1.8 \mathrm{~K}$ und thermischer Population ist daher schon bei relativ kleinen Feldern ausschließlich ZPL 1 beobachtbar.

\section{Zusammenfassung}

In diesem Kapitel wurde gezeigt, dass in den vorliegenden GaMnN-Proben tatsächlich eine, wenn auch schwache, Kopplung der Ladungsträger mit den Mn-Atomen erreicht wird. Weiterhin konnte durch die magneto-optischen Messungen die Gültigkeit des Modells von Marcet et al. [37] bestätigt werden und damit das Modell von Wolos et al. [35] widerlegt werden. Das Mn-Atom unterliegt in GaN also einem dynamischen Jahn-Teller-Effekt. 


\section{Weitere GaN-basierte DMS}

In Kapitel 2.3.2 wurden bereits die theoretischen Grundlagen angesprochen, die eine magnetische Kopplung der Fremdatome in der GaN-Matrix vorhersagen. Dabei werden vor allem die Übergangsmetalle der vierten Periode (3d-Metalle) betrachtet, aber auch seltene Erden und dort besonders das Gadolinium. Im Rahmen dieser Arbeit wurden außer von GaMnN auch die optischen Eigenschaften von GaCrN und GaGdN untersucht, um so charakteristische Gemeinsamkeiten und Unterschiede aufzudecken.

\subsection{GaCrN}

Chrom ist im Periodensystem der direkte Nachbar von Mangan, es besitzt nur ein d-Elektron weniger. In der GaN-Matrix wird es ebenso substitutionell auf dem GaGitterplatz eingebaut [153] und kann in den Ladungszuständen $\mathrm{Cr}^{2+}, \mathrm{Cr}^{3+}$ und $\mathrm{Cr}^{4+}$ existieren, wobei das $2+/ 3+$ Umladungsniveau nahezu resonant mit der Leitungsbandkante liegt [154]. Damit ist Cr ebenso wie Mn eine tiefe Störstelle, die zusätzlich intra-3dÜbergänge zeigen kann.

\subsubsection{Wachstumsbedingungen}

Die GaCrN-Proben wurden im Rahmen einer Zusammenarbeit mit dem Forschungszentrum Jülich von Nicoleta Kaluza und Yong Suk Cho hergestellt, ausführliche Details dazu finden sich in [155]. Das Wachstum erfolgt mittels MOCVD, das Probendesign ist in Abbildung 7.1 abgebildet. Dabei wird auf dem Saphir-Substrat zuerst eine GaNNukleationsschicht bei niedrigen Temperaturen gewachsen $\left(550^{\circ} \mathrm{C}\right)$ und danach ein dicker GaN-Puffer unter optimierten Wachstumsbedingungen, das bedeutet eine Wachstumstemperatur von $1125^{\circ} \mathrm{C}$ und ein V/III-Verhältnis von 877. Das entspricht der Basis für die hochqualitativen GaMnN-Schichten, wie sie in den vorhergehenden Kapiteln diskutiert wurden. Danach wird nun allerdings ohne Unterbrechung die GaCrN-Schicht gewachsen. Als Präkursor wird Trimethyl-Gallium (TMGa) und Ammoniak $\left(\mathrm{NH}_{3}\right)$ für die GaNSchichten benutzt, als Chromquelle dient zusätzlich Chromocen $\left(\mathrm{Cp}_{2} \mathrm{Cr}\right)$. Wie auch beim MBE-Wachstum sind hier die entscheidenden Parameter die Wachstumstemperatur sowie das relative Chromangebot.

Bei hohen Wachstumstemperaturen wächst das $\mathrm{GaCrN}$ in einer wabenförmigen Struktur, mit tiefen Löchern in der Schicht. Erst bei Temperaturen von $1050^{\circ} \mathrm{C}$ und niedriger ist eine glatte Oberflächenmorphologie beobachtbar. Auch ist für diese niedrigen Temperaturen der Cr-Einbau bei gleichem Angebot nahezu konstant, während er bei hohen Temperaturen deutlich absinkt, vergleichbar mit den Erfahrungen bei GaMnN. Die ab- 


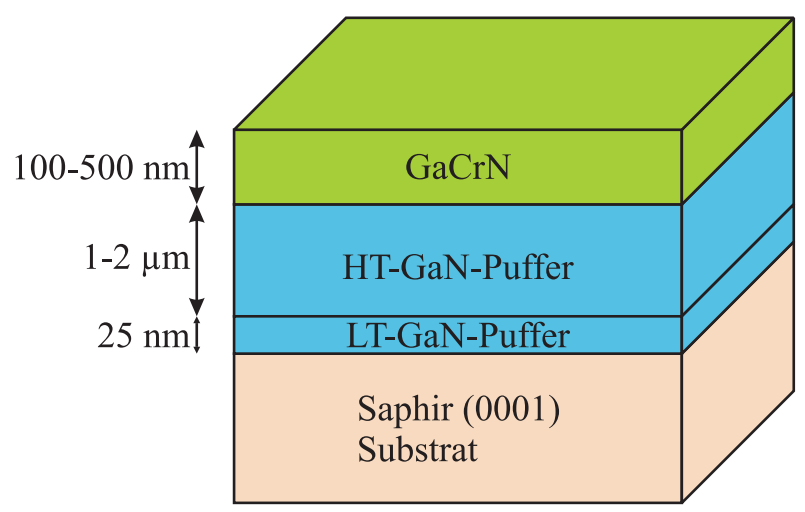

Abbildung 7.1: Aufbau der GaCrN-Proben

soluten Cr-Konzentrationen bewegen sich bei den hier vorgestellten Proben im PromilleBereich, Ausscheidungen sind nicht detektierbar, weder in Röntgenmessungen noch in TEM-Aufnahmen. Gleichzeitig ist mit diesen Charakterisierungen eine hohe strukturelle Qualität, vergleichbar mit dem GaN-Puffer, nachweisbar. Alle GaCrN-Proben zeigten Ferromagnetismus bei Raumtemperatur.

\subsubsection{PL vom UV bis sichtbaren Bereich}

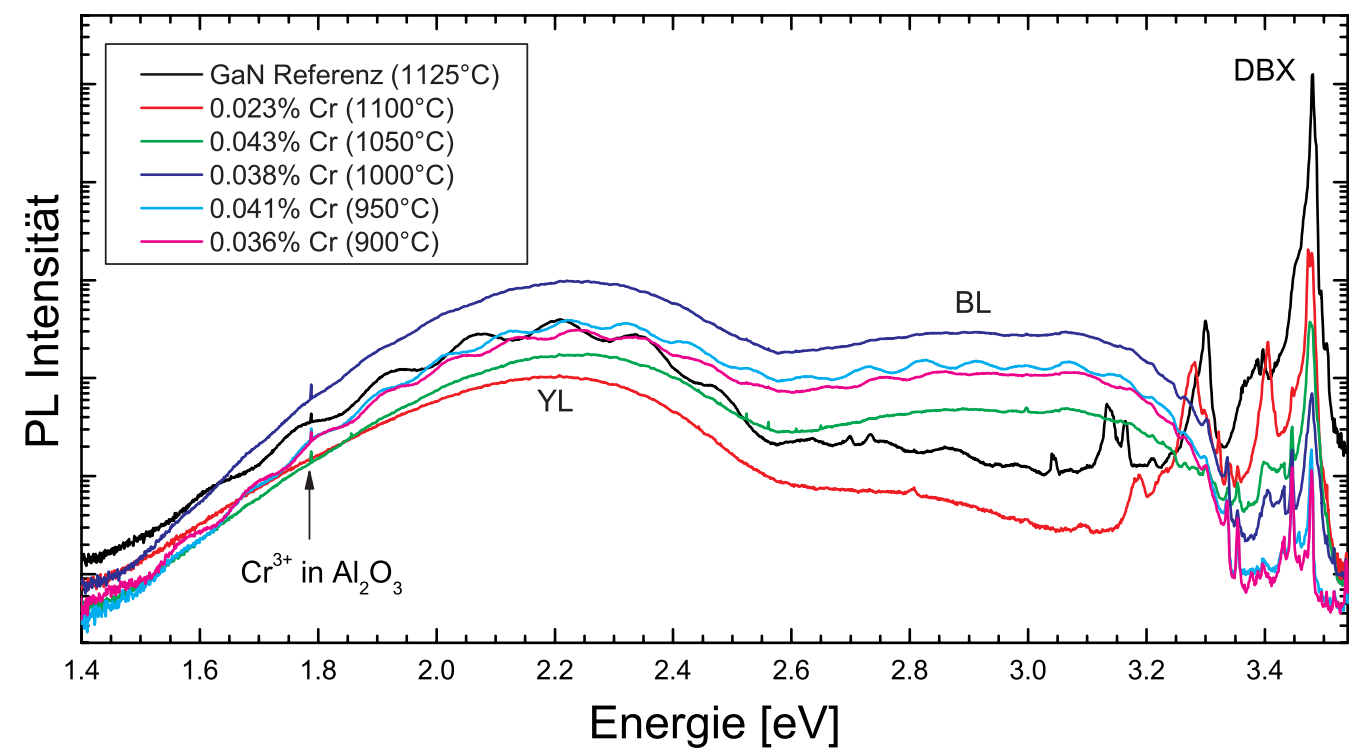

Abbildung 7.2: PL-Spektren von GaCrN-Proben mit unterschiedlichen Wachstumstemperaturen. Bei niedrigeren Temperaturen und damit steigendem Cr-Gehalt wird die bandkantennahe Lumineszenz geschwächt, während gleichzeitig eine blaue Lumineszenz (BL) entsteht.

GaCrN bei unterschiedlichen Wachstumstemperaturen Abbildung 7.2 zeigt PLSpektren einer GaN-Referenzprobe sowie mehrerer GaCrN-Proben, die bei unterschied- 
lichen Temperaturen gewachsen wurden. Die Referenzprobe zeigt die charakteristischen Signale, wie sich bereits in Kapitel 6.1 beschrieben wurden. Das DBX dominiert dabei die bandkantennahe Lumineszenz, während im Sichtbaren eine ausgeprägte gelbe Lumineszenz vorhanden ist. GaCrN bei $1100^{\circ} \mathrm{C}$ zeigt mehrere charakteristische Abweichungen. Über den gesamten Spektralbereich sinkt die PL-Intensität, vergleichbar mit den GaMnNSchichten. Besonders stark ist dieser Effekt nahe der Bandkante beobachtbar. Des weiteren verschwinden die Fabry-Perot-Oszillationen, die die gelbe Lumineszenz der Referenzprobe modulieren. Dies zeigt eine Zunahme der Oberflächenrauigkeit, da diese Oszillationen nur bei auf makroskopischer Skala glatten Proben beobachtbar sind. Wird nun die Wachstumstemperatur weiter erniedrigt, so kann man eine weitere stetige Abnahme der exzitonischen Lumineszenz nahe der Bandkante beobachten. Gleichzeitig erscheint im Bereich von $2.6-3.3 \mathrm{eV}$ ein neues Band, die so genannte blaue Lumineszenz (BL), und die gelbe Defekt-Lumineszenz gewinnt an Intensität. Sowohl BL als auch YL zeigen bei $1000^{\circ} \mathrm{C}$ ihre maximale Intensität, um bei noch niedrigeren Temperaturen wieder schwächer zu werden. Bei $950^{\circ} \mathrm{C}$ und $900^{\circ} \mathrm{C}$ treten wieder Fabry-Perot-Oszillationen, was auf eine glatte Oberflächenmorphologie hindeutet. Dies ist in Übereinstimmung mit SEM-Aufnahmen, die für diese niedrigen Temperaturen sehr glatte Oberflächen auf der Mikrometer-Skala zeigen, während die hohen Temperaturen wabenförmige Muster mit tiefen Löchern aufweisen.

Während die Ursache der YL in der Literatur als geklärt gelten darf (siehe Kapitel 4.1.3), wird eine blaue Lumineszenz im Bereich um 2.7 - 3.0 eV nach wie vor kontrovers diskutiert [73]. Eine Interpretation ordnet sie einem Komplex eines ÜbergangsmetallAtoms mit einem intrinsischen Defekt zu, da diese Lumineszenz beispielsweise in GaMnN $[142,156,157,140,158]$ und $\mathrm{GaCrN}[159,160,161,162,163,164,165]$ gefunden wurde. Die in Abbildung 7.2 gezeigten Messungen könnten diese Zuordnung unterstützen, da in der Referenzprobe und der schwach dotierten $1100^{\circ} \mathrm{C}$-Probe keine BL nachweisbar war, während in den höher dotierten Niedrig-Temperatur-Proben dieses Band deutlich wurde. In letzteren Proben ist der Cr-Gehalt nahezu gleich, ebenso wie die Intensität der BL im Vergleich zur YL. Schwankungen der absoluten Intensität können durch eine unterschiedliche Zahl nicht-radiativer Defekte verursacht werden und sind daher nicht so aussagekräftig. Allerdings ist diese Korrelation der Cr-Konzentration mit der BL nicht als bewiesen anzusehen, da gleichzeitig durch die Variation der Wachstumstemperatur Einflüsse auf die intrinsischen Defekte zu erwarten sind, die ebenso als Ursache für die BL in Frage kommen. Eine weitere Interpretation ordnet die BL nämlich einem DAP-Übergang zu, wobei als Akzeptor sowohl intrinsische Defekte als auch ungewollte Kontaminationen in Frage kommen ([166] und dortige Referenzen).

Bei $1.788 \mathrm{eV}$ ist in allen Proben aus Abbildung 7.2 eine scharfe Linie beobachtbar, auch in der Referenzprobe. Sie wird einem intra-3d-Übergang von $\mathrm{Cr}^{3+}$ in Saphir zugeordnet [167], dem gleichen Übergang, der für den ersten Rubinlaser verwendet wurde [168]. Da diese Linie durch Licht der YL effektiv angeregt werden kann [169] und GaN in diesem Spektralbereich nahezu durchsichtig ist, kann sie trotz der hohen Schichtdicke in den vorliegenden Proben detektiert werden. Andere Proben, deren Saphir-Substrat von einem anderen Hersteller kam, zeigten diese Linie nicht, es handelt sich also nicht um eine CrDiffusion aus der GaCrN-Schicht oder eine Kontamination der MOCVD-Anlage, sondern um eine Verunreinigung des kommerziell erworbenen Substrats. 


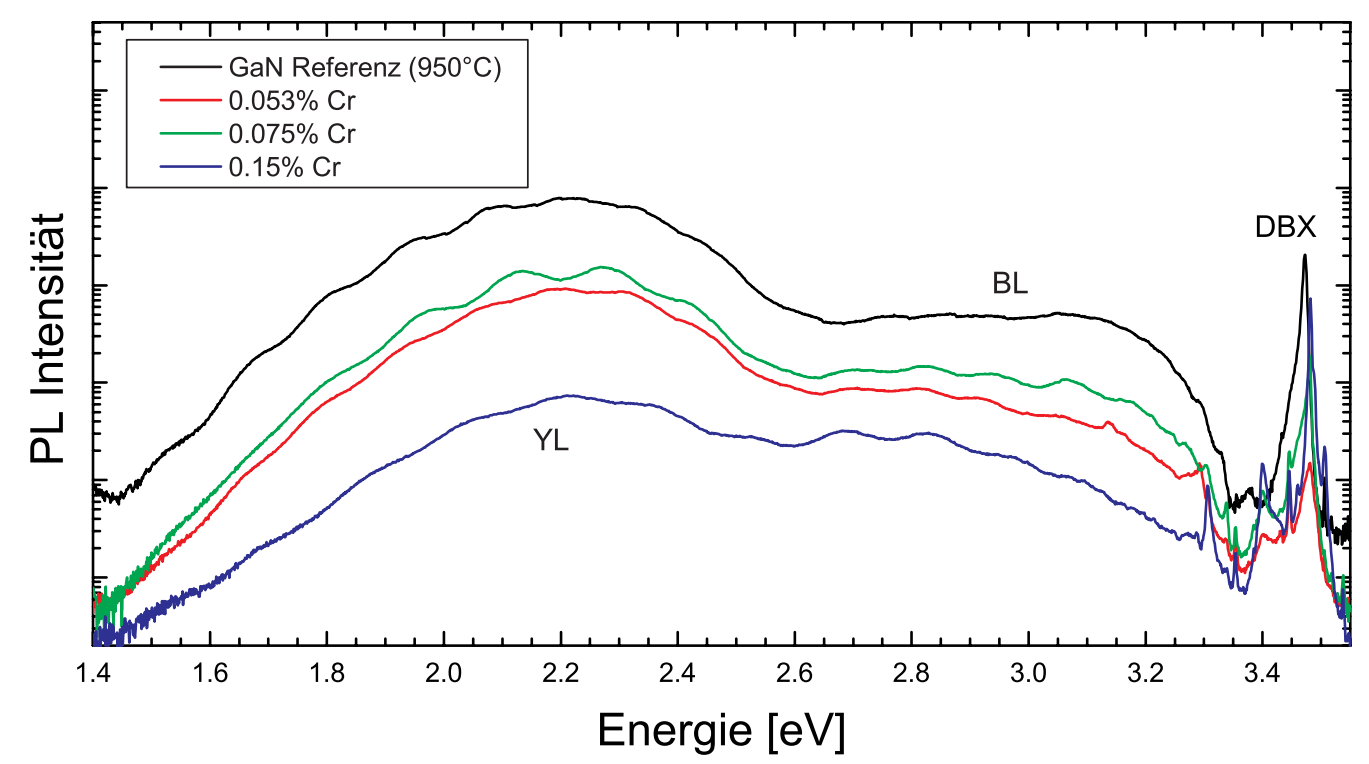

Abbildung 7.3: PL-Spektren einiger bei $950^{\circ} \mathrm{C}$ gewachsenen $\mathrm{GaCrN}-\mathrm{Proben}$ mit unterschiedlichen $\mathrm{Cr}-$ Gehalt. Die blaue Lumineszenz (BL) tritt schon in der GaN-Referenzprobe auf und wird durch einen höheren Cr-Gehalt ebenso wie die Lumineszenz im restlichen Spektralbereich geschwächt.

GaCrN bei konstanter Wachstumstemperatur und variablem Cr-Angebot Der genaue Einfluss des $\mathrm{Cr}$ in GaCrN kann nur in Proben untersucht werden, die bei ansonsten konstanten Wachstumsbedingungen verschiedene Cr-Konzentrationen aufweisen. Einige solcher Proben sind in Abbildung 7.3 abgebildet zusammen mit einer GaN-Referenzprobe, die bei der selben niedrigen Wachstumstemperatur von $950^{\circ} \mathrm{C}$ hergestellt wurde. Hier zeigt sich nun klar, dass die BL nicht eindeutig auf einen Cr-spezifischen Defekt zurückgeführt werden kann, da die undotierte Referenzprobe eine sehr ausgeprägte BL aufweist mit der gleichen Form wie alle anderen Cr-dotierten Proben (siehe zum Vergleich auch Abbildung 7.2). Es muss also ein Cr-unabhängiger Defekt dafür verantwortlich sein, der durch die niedrigen Wachstumstemperaturen bevorzugt auftritt.

Die bandkantennahe Lumineszenz kann in dieser Serie nicht konsequent analysiert werden, da die Proben mit hoher Cr-Konzentration eine deutlich verringerte Wachstumsrate zeigten und daher erheblich dünnere Schichten ergaben. Die mit $0.15 \%$ höchstdotierte Probe (siehe Abbildung 7.3) ist nur etwa $100 \mathrm{~mm}$ dick, weshalb hier die GaN-Pufferschicht „durchscheint". Dies liegt daran, dass dieses GaN unter optimierten Bedingungen gewachsen wurde und so eine mehrere Größenordnungen stärkere Bandkantenlumineszenz besitzt. Die Eindringtiefe (1/e-Wert) des HeCd-Laser liegt bei etwa $83 \mathrm{~nm}$, weshalb noch ein erheblicher Bruchteil den GaN-Puffer erreicht. Für die austretende Lumineszenz existiert ein ähnlicher Verlust durch die Selbstabsorption in der GaCrN-Schicht, so dass die bandkantennahe Lumineszenz der Pufferschicht pro $100 \mathrm{~nm}$ GaCrN-Schichtdicke um etwa eine Größenordnung unterdrückt wird. Erst bei mehreren $100 \mathrm{~nm}$ Dicke erhält man daher reine GaCrN-Spektren. Bei den dünnen Schichten zeigt erst ein Vergleich mit der Referenzprobe aus Abbildung 7.2 die spezifischen Signale von GaCrN.

Detaillierte Analyse der blauen Lumineszenz In Abbildung 7.4 ist der bandkantennahe Bereich und die hochenergetische Flanke der BL für eine bei $1000^{\circ} \mathrm{C}$ gewachsene Probe 
im Detail abgebildet. Die exzitonische Lumineszenz ist bei diesen niedrigen Temperaturen verbreitert und es treten wie in GaMnN Raman-Linien, also Phononen-Repliken der anregenden Laserlinie auf.

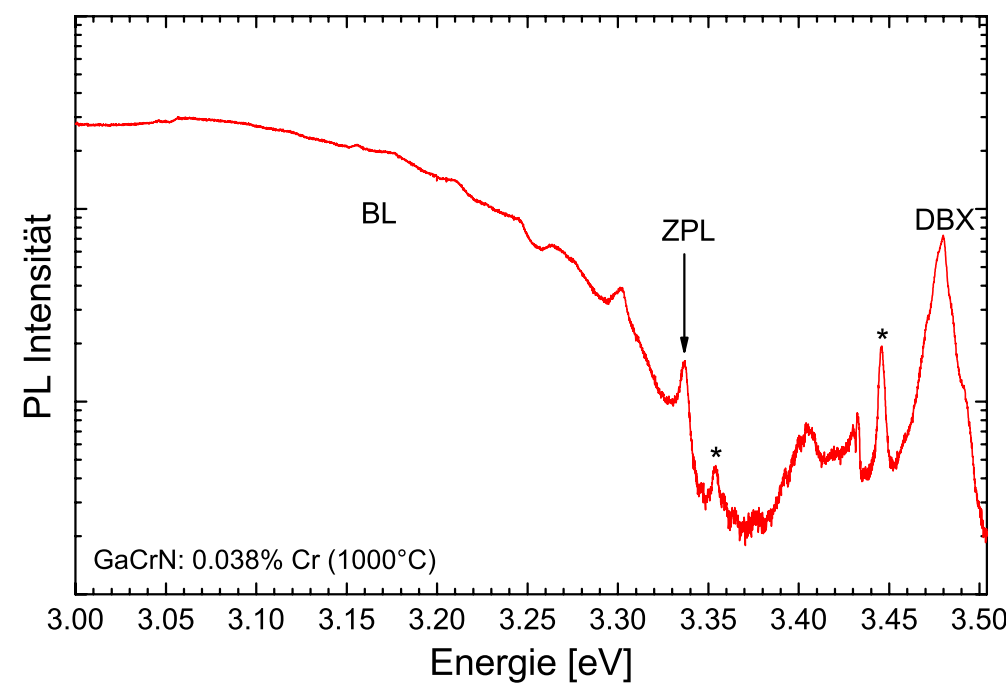

Abbildung 7.4: Detail-Spektrum der Bandkante und der hochenergetischen Flanke der BL einer repräsentativen Probe. Mit „*“ werden Raman-Linien bezeichnet, die analog dem GaMnN auch im GaCrN auftreten.

Die hochenergetische Flanke der BL wird von einer relativ scharfen Linie bei $3.337 \mathrm{eV}$ begrenzt, die hier als ZPL bezeichnet wird, da sie nur in Verbindung mit der BL auftritt und daher als deren Null-Phononen-Linie identifiziert wird. Außerdem zeigt die BL eine charakteristische Struktur nahe dieser ZPL, die sich im weiteren Verlauf abschwächt. Dies ist eine typische Struktur für einen Übergang mit starker Elektron-Phonon-Kopplung. Leistungsabhängige Messungen zeigen keinerlei Veränderung der Form der BL und insbesondere keine Verschiebung der energetischen Position. Die Intensität erhöht sich superlinear mit steigender Anregungsleistung und läuft dabei parallel zur exzitonischen Lumineszenz.

In Abbildung 7.5a) sind schließlich temperaturabhängige Messungen an der BL abgebildet. Bei Temperaturerhöhung sinkt die Intensität der BL (sowohl absolut als auch relativ zur YL oder Bandkante). Außerdem ist eine Rotverschiebung beobachtbar, die mit $170 \mathrm{meV}$ von $16 \mathrm{~K}$ auf $150 \mathrm{~K}$ viel größer als die Abnahme der Bandlücke von GaN ist. Um eine Erklärung für dieses Verhalten zu finden, wurde der Spektralbereich von 2.0 - 3.3 eV durch Gauß-Funktionen angepasst. Dabei zeigt sich, dass sich die gelbe Lumineszenz wie erwartet durch ein einziges Band mit Zentrum bei $2.2 \mathrm{eV}$ beschreiben lässt, eine gute Anpassung der blauen Lumineszenz aber nur mit zwei Gauß-Funktionen erreicht wird, wie in Abbildung 7.5b) dargestellt. Die einzelnen Bänder zeigen nur eine sehr schwache Rotverschiebung bei Temperaturerhöhung. Die Intensitäten bleiben bis etwa $50 \mathrm{~K}$ nahezu konstant und fallen dann stark ab. Das Band bei $3.1 \mathrm{eV}$ verliert dabei so schnell an Intensität, dass es oberhalb von $76 \mathrm{~K}$ nicht mehr nachweisbar ist. Das Band bei $2.9 \mathrm{eV}$ 

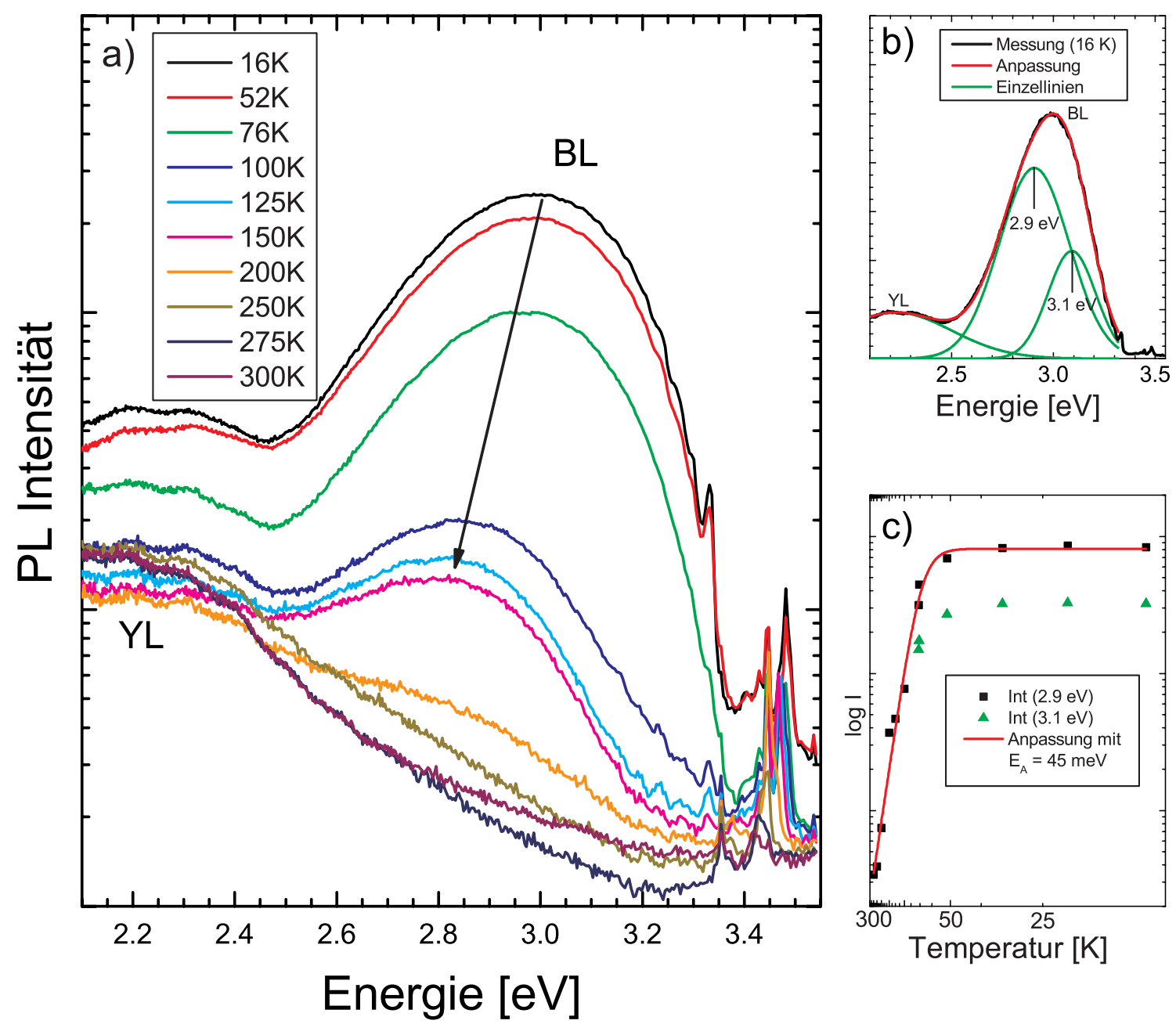

Abbildung 7.5: a) Temperaturabhängige PL-Spektren der BL zeigen eine Rotverschiebung bei Temperaturerhöhung. b) Auflösung der BL in zwei Bänder mittels Anpassung von Gauß-Funktionen. c) Temperaturabhängigkeit der Intensitäten der beiden Bänder bei $2.9 \mathrm{eV}$ und $3.1 \mathrm{eV}$. Das höherenergetische Band wird nur bei tiefen Temperaturen gefunden, das niederenergetische zeigt eine Aktivierungsenergie von $45 \pm 4 \mathrm{meV}$ (nach Gleichung 7.1)

lässt sich durch

$$
I(T)=\frac{I_{0}}{1+B \exp \left(\frac{E_{A}}{k T}\right)}
$$

anpassen. Diese Funktion beschreibt den Intensitätsverlust eines Bandes mittels thermischer Anregung eines Konkurrenzprozesses. Dabei ist $E_{A}$ die Aktivierungsenergie des Konkurrenzprozesses, $I_{0}$ die Intensität bei tiefen Temperaturen und $B$ hängt vom Verhältnis der Lebensdauern der beiden Prozesse ab. Es ergibt sich $E_{A}=45 \pm 4 \mathrm{meV}$. Subashchandran et al. [165] finden für die BL von GaCrN eine Aktivierungsenergie von $E_{A} \approx 340 \mathrm{meV}$, allerdings liegt die dort berichtete BL bei niedrigeren Energien (ungefähr 2.7-3.0 eV) und zeigt in der Leistungsabhängigkeit eine für DAP-Übergänge typische Blauverschiebung. Dasselbe trifft auf die von Reshchikov et al. [166] analysierte BL von undotiertem $\mathrm{GaN}$ zu, wobei $E_{A} \approx 380 \mathrm{meV}$ gemessen wurde. Die im Rahmen dieser Ar- 
beit analysierten $\mathrm{GaCrN}$-Proben zeigen weder eine Blauverschiebung der BL bei höheren Anregungsleistungen, noch die für flache Akzeptoren in GaN typische Aktivierungsenergie von 300-400 meV. Daraus kann geschlussfolgert werden, dass die beobachtete BL kein DAP-Übergang ist. Die beobachtete Aktivierungsenergie von $E_{A}=45 \pm 4 \mathrm{meV}$ passt eher in den Bereich exzitonischer Lumineszenzen oder flacher Donatoren. Auch die beobachtete ZPL bei $3.337 \mathrm{eV}$ deutet eher auf tief gebundene Exzitonen hin. Allerdings werden bei vergleichbaren Übergängen (siehe z. B. Abbildung 6.2, die mit BX bezeichneten Signale in GaMnN) typischerweise keine scharfen ZPL mit einem breiten phononischen Seitenband beobachtet. Eine genaue Zuordnung zu einem bestimmten Defekt ist daher an dieser Stelle noch nicht möglich, der Zusammenhang mit intrinsischen Defekten ist an Hand der in Abbildung 7.3 präsentierten Daten aber gesichert.

\subsubsection{PL im Infraroten}

Zum Auffinden möglicher intra-3d-Übergänge wurden PL-Spektren bis zum Detektionslimit des CCD-Chips aufgenommen, welches bei etwa $1.1 \mathrm{eV}$ liegt. Wie in Abbildung 7.6 zu sehen ist, konnte dabei in GaCrN eine scharfe Linie bei $1.193 \mathrm{eV}$ gefunden werden, die in den GaN-Referenzproben nicht auftritt. Gleichzeitig ist in allen untersuchten Proben bei $1.300 \mathrm{eV}$ eine Linie mit einem ausgedehnten phononischen Seitenband vorhanden, die bereits in den GaMnN-Proben auf MOCVD-gewachsenem GaN-Puffer gefunden wurde (siehe Kapitel 6.2.3). Sie wird einem intra-3d-Übergang von $\mathrm{Fe}^{3+}$ zugeordnet [132].

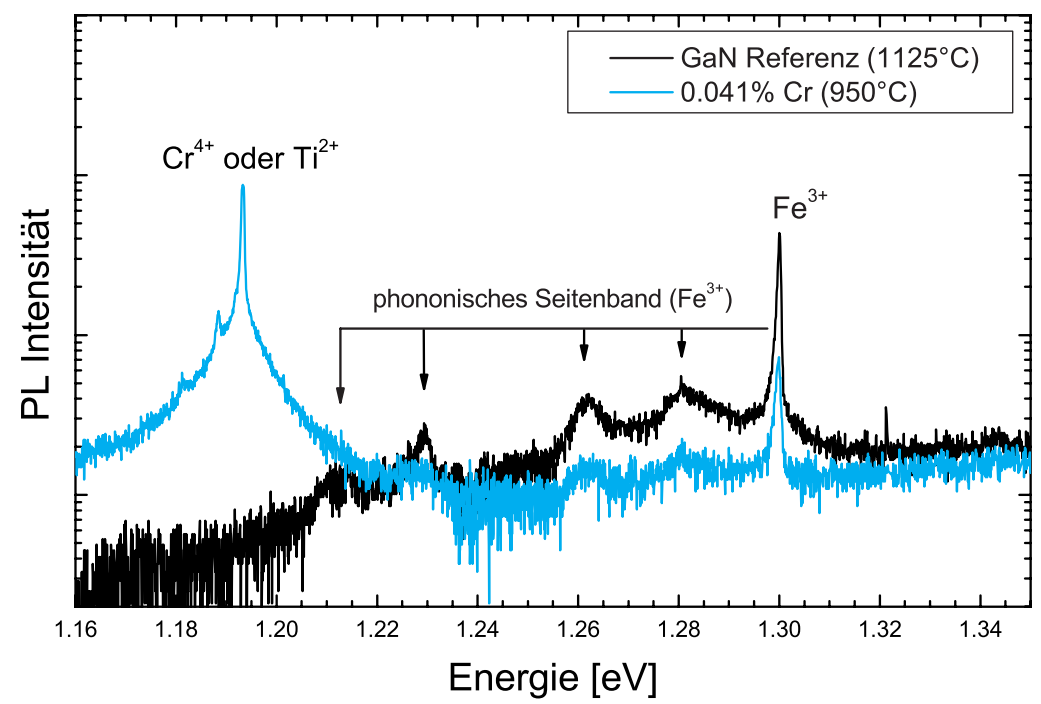

Abbildung 7.6: PL-Spektren im Infraroten einer bei $950^{\circ} \mathrm{C}$ gewachsenen GaCrN-Probe und einer GaNReferenz. Bei $1.300 \mathrm{eV}$ ist ein $\mathrm{Fe}^{3+}$-Übergang sichtbar mit einem phononischen Seitenband. Nur die Cr-dotierte Probe zeigt eine weitere ausgeprägte Linie bei $1.193 \mathrm{eV}$.

Die Linie bei $1.193 \mathrm{eV}$ ist in der Literatur bekannt und wurde Mitte der 90er Jahre im Wesentlichen von zwei Gruppen charakterisiert. Baur et al. [132, 170], die diese Linie erstmals in nominell undotierten Proben beobachteten, ordnen sie einem intra-3d-Übergang eines $\mathrm{d}^{2}$-Elektronensystems zu. Dieser Zustand existiert in GaN nur bei $\mathrm{Ti}^{2+}, \mathrm{V}^{3+}$ und 
$\mathrm{Cr}^{4+}$. Der $\mathrm{V}^{3+}$-Übergang wurde bereits einer Linie bei $0.931 \mathrm{eV}$ zugeordnet [170]. Baur et al. kommen zu dem Schluss, dass es sich um einen $\mathrm{Cr}^{4+}$-Übergang handelt. Dies wird mit Details der Kristallfeldaufspaltung begründet, sowie der Tatsache, dass in diesen Arbeiten eine Verstärkung dieser Lumineszenz bei p-leitenden Schichten beobachtet wurde. Heitz et al. [171] fanden sie ebenfalls in nominell undotierten Proben und konnten sie mittels magneto-optischer Messungen klar einem intra-3d-Übergang eines $d^{2}$-Elektronensystems zuordnen. Auf Grund von Anregungsspektroskopie und der Tatsache, dass ihre Proben n-leitend waren, schlossen Heitz et al. schließlich auf $\mathrm{Ti}^{2+}$ als Ursache für die $1.193 \mathrm{eV}$ Lumineszenz. Außerdem konnten sie die wesentliche Nebenlinie nicht beobachten, mit der Baur et al. ihre Zuordnung rechtfertigten.

Neben diesen widersprüchlichen experimentellen Ergebnissen wurden von Gerstmann et al. [154] theoretische Berechnungen mittels Dichtefunktionaltheorie durchgeführt. Dabei wurden die elektronischen Eigenschaften verschiedener Übergangsmetalle in den Gruppe-III-Nitriden verglichen. Sie kommen dabei zu dem Ergebnis, dass Titan in GaN nur in den Ladungszuständen $\mathrm{Ti}^{3+}$ und $\mathrm{Ti}^{4+}$ existiert, das $2+/ 3+$ Umladungsniveau liegt demnach oberhalb der Leitungsbandkante. Daher folgen sie dem ursprünglichen Vorschlag von Baur et al. und schlagen $\mathrm{Cr}^{4+}$ als Ursache dieser Lumineszenz vor. In n-leitendem GaN müsste dieser Lumineszenz dann eine Umladung während des Anregungsprozesses vorangehen, da hier $\mathrm{Cr}^{3+}$ im Grundzustand vorläge. Ein vergleichbarer Prozess wurde beispielsweise bei Fe in GaN diskutiert (von Heitz et al. [172]), und kann auch für $\mathrm{Cr}$ nicht ausgeschlossen werden.

Es soll an dieser Stelle noch einmal betont werden, dass die früheren Veröffentlichungen über die $1.193 \mathrm{eV}$-Lumineszenz alle auf nominell undotierten GaN-Proben beruhen, bei denen der zu Grunde liegende Defekt als ungewollte Kontamination in der Probe vorhanden war. Mittlerweile können qualitativ wesentlich bessere Proben hergestellt werden und diese Kontaminationen treten deutlich seltener auf. Lediglich Eisen ist auf Grund der $\mathrm{Fe}^{3+}$-Linie in nominell undotierten Proben des im Rahmen dieser Arbeit untersuchten MOCVD-gewachsenen GaN nachweisbar. Das Auftreten der intensiven Linie bei $1.193 \mathrm{eV}$ kann daher klar mit dem Cr-Präkursor korreliert werden, da sie nur in GaCrN-Proben auftritt. Dies ist ein deutlicher Hinweis auf Cr als Ursache dieser Lumineszenz.

Allerdings wäre auch eine Verunreinigung eben dieses Cr-Präkursors denkbar, da bei einem Cr-Angebot im Promille-Bereich auch eine schwache Verunreinigung mit Ti zu Konzentrationen im GaN führen könnte, die für die beobachtete Intensität ausreichen. Als Präkursor wurde Chromocen (bis-Cyclopentadienylchrom) benutzt, eine metallorganische Verbindung aus zwei aromatischen Fünfringen mit einem Chrom-Atom in der Mitte, dessen d-Elektronen mit dem $\pi$-System der Aromaten eine Bindung eingehen [173]. Diese Metallocene existieren für die meisten Übergangsmetalle, so dass je nach Reinheit des Ausgangsmaterials Verunreinigungen der Substanz auftreten. Allerdings gehört Ti als Metall der 2. Nebengruppe zu den Ausnahmen, die keine stabile Metallocen-Verbindung in dieser Konfiguration bilden können [174]. Damit ist Ti als Verunreinigung des CrPräkursors sehr unwahrscheinlich. Auf Grund dieser Argumente kann die Lumineszenz bei $1.193 \mathrm{eV}$ mit großer Sicherheit einem intra-3d-Übergang von $\mathrm{Cr}^{4+}$ in $\mathrm{GaN}$ zugeordnet werden.

Die Probenserie mit unterschiedlichem Cr-Gehalt wurde auch im Infraroten untersucht. 
Dabei zeigt sich, dass eine Erhöhung der Cr-Konzentration nicht zu einer Erhöhung der Signalstärke führt, sondern im Gegenteil zu einer Abnahme, wie in Abbildung 7.7a) ersichtlich. Die Proben sind dabei (von oben nach unten) nach steigendem Cr-Angebot beim Wachstum angeordnet, die angegebenen Konzentrationen sind mittels SIMS ermittelt. Die anscheinend ,falsche" Reihenfolge der Konzentrationen in der Legende ist dabei eher auf Ungenauigkeiten in der SIMS-Messung zurückzuführen als auf ein verändertes Einbauverhalten.
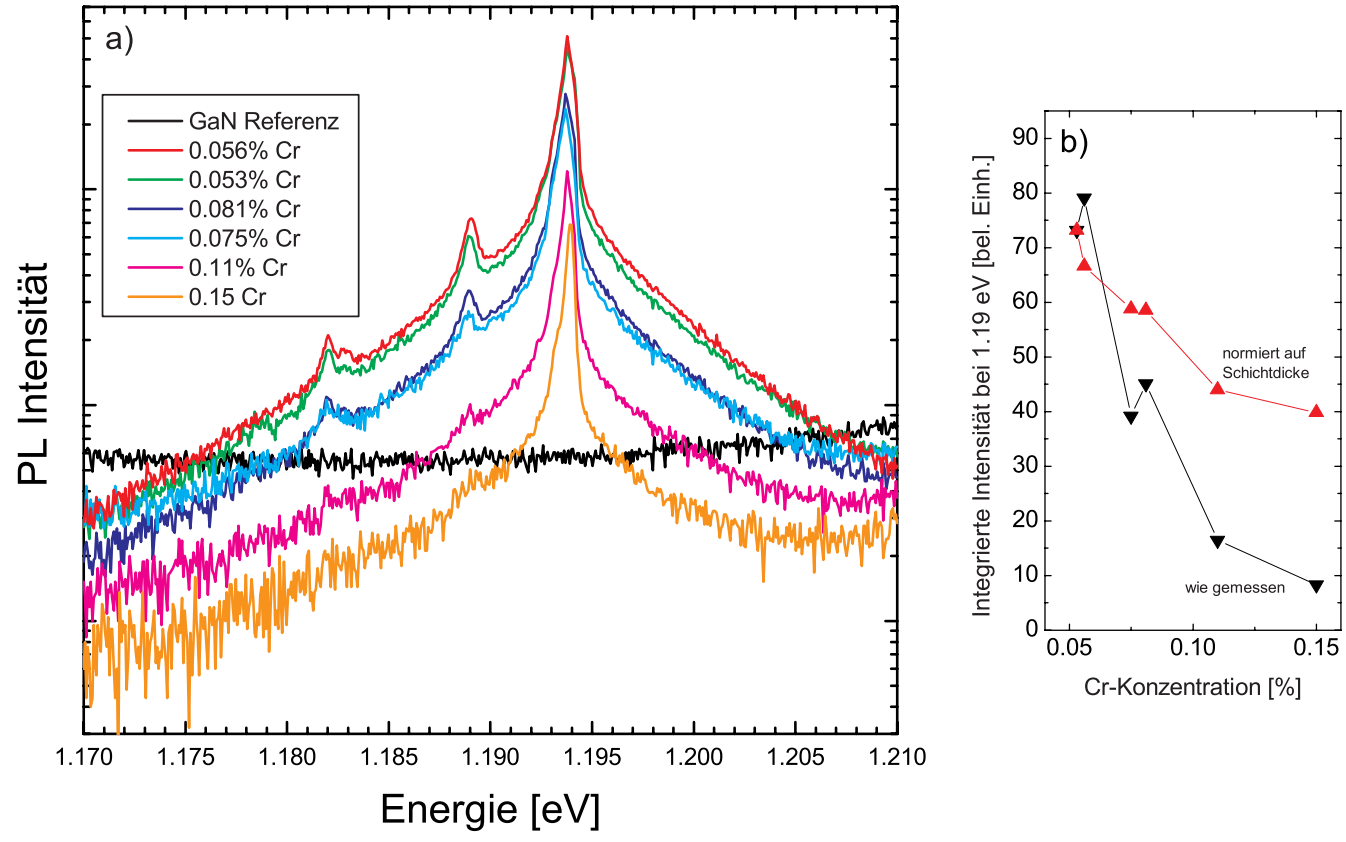

Abbildung 7.7: a) PL-Spektren im Infraroten der bei $950^{\circ} \mathrm{C}$ gewachsenen GaCrN-Proben mit unterschiedlichem Cr-Gehalt. b) Die Intensität skaliert nicht mit dem Cr-Gehalt, sondern nimmt mit höherer Cr-Konzentration ab.

Die geringere Schichtdicke bei höherem Cr-Gehalt wurde bereits in Kapitel 7.1.2 angesprochen. Tatsächlich nimmt die GaCrN-Schichtdicke parallel zur sinkenden Intensität der intra-3d-Linie ab. Selbst wenn man diesen Effekt berücksichtigt, indem man die Intensität auf die Schichtdicke normiert, ergibt sich immer noch eine Abnahme der Intensität mit steigender Cr-Konzentration, wie in Abbildung 7.7b) gezeigt. Ähnlich wie in GaMnN funktioniert die Anregung des intra-3d-Übergangs also bei niedrigen Cr-Konzentrationen am besten, während hohe Cr-Konzentrationen durch zusätzliche Defekte andere Rekombinationskanäle bereitstellen, was die Lumineszenz schwächt.

\section{Zusammenfassung}

In diesem Kapitel wurde gezeigt, dass in $\mathrm{GaCrN}$ sowohl eine blaue Lumineszenz als auch ein intra-3d-Übergang beobachtbar sind. Die blaue Lumineszenz konnte allerdings chromunabhängigen intrinsischen Defekten zugeordnet werden, die durch die Wachstumsbedingungen verursacht wurden. Der intra-3d-Übergang wurde in der Literatur kontrovers diskutiert und kann nun endgültig dem $d^{2}$-System von $\mathrm{Cr}^{4+}$ zugeordnet werden. 


\subsection{GaGdN}

Gadolinium zeigt im Vergleich zu den bisher diskutierten 3d-Übergangsmetallen einige wesentliche Unterschiede. Es ist ein Element der 6. Periode und daher deutlich größer und schwerer als das Gallium, welches es im GaN-Gitter ersetzt, wie EXAFS-Messungen von Hashimoto et al. zeigen [175]. Die Elektronenkonfiguration ist [Xe] $6 \mathrm{~s}^{2} 5 \mathrm{~d}^{1} 4 \mathrm{f}^{7}$, wobei im Ladungszustand $\mathrm{Gd}^{3+}$ die s- und das d-Elektron abgegeben werden. Damit ist $\mathrm{Gd}$ isoelektronisch zu Ga. Theoretische Rechnungen [15, 50] sagen sogar voraus, dass Gd keine Zustände innerhalb der Bandlücke von GaN verursacht, es wäre demnach elektrisch neutral. Intra-4f-Übergänge (analog den intra-3d-Übergängen der Übergangsmetalle) des $\mathrm{Gd}^{3+}$-Ions sind bekannt, beispielsweise in AlN:Gd [176]. Allerdings befindet sich der erste angeregte Zustand ${ }^{6} \mathrm{P}_{7 / 2}$ etwa $3.9 \mathrm{eV}$ oberhalb des Grundzustands ${ }^{8} \mathrm{~S}_{7 / 2}$, nahezu unabhängig von der Matrix. Es ist daher sehr unwahrscheinlich, diese Übergänge in GaGdN mit einer Bandlücke von etwa $3.5 \mathrm{eV}$ anregen oder beobachten zu können. Damit sind also keine direkten Auswirkungen des Gd-Einbaus auf die PL-Spektren zu erwarten. Indirekte Auswirkungen können sich aber durch Veränderung der Wachstumsbedingungen bei Gd-Angebot ergeben, oder zum Beispiel durch Verspannungseffekte.

\subsubsection{Wachstumsbedingungen}

Die GaGdN-Proben wurden mittels MBE auf Saphir-Substraten mit einem MOCVDgewachsenen GaN-Puffer gewachsen. Dabei wurde der Ga-Fluss auf dem Niveau gehalten, bei dem reines GaN unter stöchiometrischen Bedingungen wächst, also ohne Metall-Überschuss. Zusätzlich wurde Gd angeboten und in Konzentrationen von etwa $10^{16}-10^{21} \mathrm{~cm}^{-3}$ eingebaut. Dies wurde an Hand von SIMS-Messungen überprüft, die einen homogenen Einbau über die Schichtdicke zeigen. Es ergibt sich dabei ein exponentieller Zusammenhang zwischen der Gd-Zellentemperatur und der eingebauten Gd-Konzentration, der dazu genutzt wurde, die Konzentration nicht direkt gemessener Proben per Extrapolation zu bestimmen.

XRD zeigt eine hohe Kristallqualität (entsprechend dem GaN-Puffer) und weder mit XRD noch mit TEM sind Ausscheidungen detektierbar. Die höchste so erhaltene GdKonzentration beträgt $2.9 \%$.

\subsubsection{Bandkantennahe PL}

Bei niedrigen Gd-Konzentrationen gleichen die gemessenen PL-Spektren denen von GaNReferenzproben. Nahe der Bandkante dominiert das Donator-gebundene Exziton (DBX), aber auch schwache Linien von an strukturelle Defekte gebundenen Exzitonen sind nachweisbar. Die gelbe Lumineszenz (YL) ist vorhanden, aber schwach im Vergleich zur DBXLinie. Wie auf Grund der isoelektronischen Eigenschaften zu erwarten, ändert sich daran mit erhöhter Gd-Konzentration nichts. Nur bei der höchst dotierten Probe ist außer der bandkantennahen Lumineszenz kein weiteres Signal mehr zu detektieren, selbst die YL ist nicht nachweisbar. Dies deutet auf effektive nicht-radiative Kanäle hin. Eine weitere Interpretation ist die Abnahme der Zahl der Ga-Vakanzen, die an der YL beteiligt sind. 
Ein möglicher Mechanismus dafür wird im Verlauf dieses Kapitels diskutiert werden. Es konnten keine internen Übergänge der $\mathrm{Gd}^{3+}$-Ionen detektiert werden, wie sie etwa von Lipinska et al. [177] als Übergang zwischen zwei angeregten Niveaus bei $1.78 \mathrm{eV}$ vorgeschlagen wurden. Choi et al. detektieren bei $1.90 \mathrm{eV}$ eine scharfe Linie, die sie einem transienten Zustand von $\mathrm{Gd}^{2+}$ zuordnen $[162,178]$. Weder dieser, noch der $\mathrm{Gd}^{3+}$-Übergang ${ }^{6} \mathrm{P}_{7 / 2} \rightarrow{ }^{8} \mathrm{~S}_{7 / 2}$, wie er von Lipinska et al. [177] bei etwa $3.3 \mathrm{eV}$ möglicherweise beobachtet wurde, war in den vorliegenden Proben sichtbar. Alle diese intra-4f-Übergänge wurden jeweils nur von einer Gruppe beobachtet und sind daher als höchst spekulativ einzuordnen, auf Grund der bereits angesprochenen Lage der $f$-Niveaus innerhalb der GaN-Bänder und der hohen Anregungsenergie des ersten angeregten Zustands. Das Auftreten solcher Linien könnte daher möglicherweise ein Hinweis auf Gd in einer zweiten Phase sein. Das Nichtvorhandensein in den Göttinger Proben dagegen wäre dann ein Hinweis auf das Fehlen von Ausscheidungen, was auch durch XRD und TEM gezeigt wird.
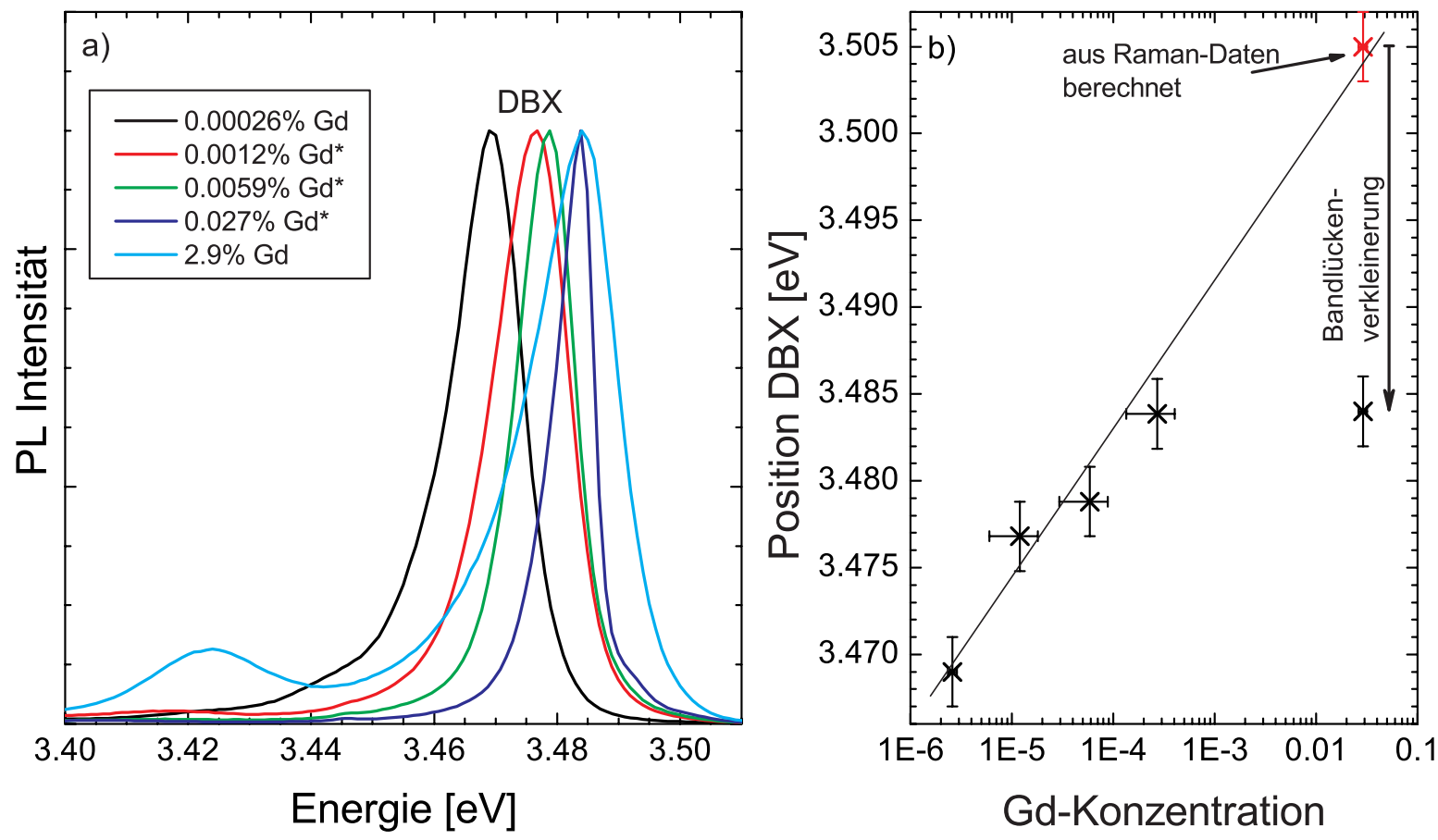

Abbildung 7.8: a) Auf das DBX-Signal normierte PL-Spektren der GaGdN-Proben mit unterschiedlicher Gd-Konzentration. Die mit "*" markierten Konzentrationsangaben sind mit einer großen Ungenauigkeit behaftet (siehe Text). b) Verschiebung des DBX-Signals mit der Gd-Konzentration. In rot die aus den Raman-Messungen (Abbildung 7.9) zu erwartende Position für die hochkonzentrierte Probe. Die Gerade ist nur als Richtlinie anzusehen.

In Abbildung 7.8a) ist ein Ausschnitt der PL-Spektren um die DBX-Linie von verschieden stark dotierten GaGdN-Proben zu sehen. Man erkennt einen Trend zu höheren Rekombinationsenergien mit steigendem Gd-Gehalt. An dieser Stelle muss allerdings darauf hingewiesen werden, dass die mit ," " bezeichneten Proben aus einer GaGdN-Serie stammen, die nicht mittels SIMS analysiert wurde und mit einer anderen Gd-Zelle hergestellt wurden. Die Konzentrationsangaben sind aus der Gd-Zellentemperatur extrapoliert, weisen aber einen erhöhten systematischen Fehler auf, da die Zellengröße etwas verschieden 
war. Dieser Fehler wurde für die Auswertung der Position der DBX-Signale in Abbildung 7.8b) auf 50\% anstelle der ansonsten vorhandenen SIMS-Ungenauigkeit von etwa 10\% geschätzt.

Die vier niedrig dotierten Proben zeigen einen eindeutigen Trend des DBX-Signals zu höheren Energien bei steigender Gd-Konzentration. Dies wird durch die eingezeichnete Gerade verdeutlicht. Allerdings fällt die Probe mit 2.9\% Gd aus diesem Bild heraus, da sie im Vergleich zur nächst niedriger dotierten Probe keine signifikante Erhöhung mehr aufweist. Eine mögliche Erklärung für die Blauverschiebung bei höheren Gd-Konzentrationen ist in einer kompressiven Verspannung zu finden. Kompressive Verspannungen verschieben die DBX-Linie zu höheren Energien, wie es hier auch beobachtet werden konnte. Sie können unabhängig von PL-Messungen auch beispielsweise durch Raman-Messungen an der $\mathrm{E}_{2}$-Mode bestimmt werden, welche ebenfalls eine Blauverschiebung bei kompressiver Verspannung aufweist. Entsprechende Messungen an niedrig dotierten Proben ergaben kein eindeutiges Ergebnis, da das Raman-Signal der GaGdN-Schicht von dem der GaNPufferschicht überlagert wird und keine klare Trennung möglich war. Bei der Probe mit $2.9 \%$ Gd ergab sich das in Abbildung 7.9 gezeigte Bild.

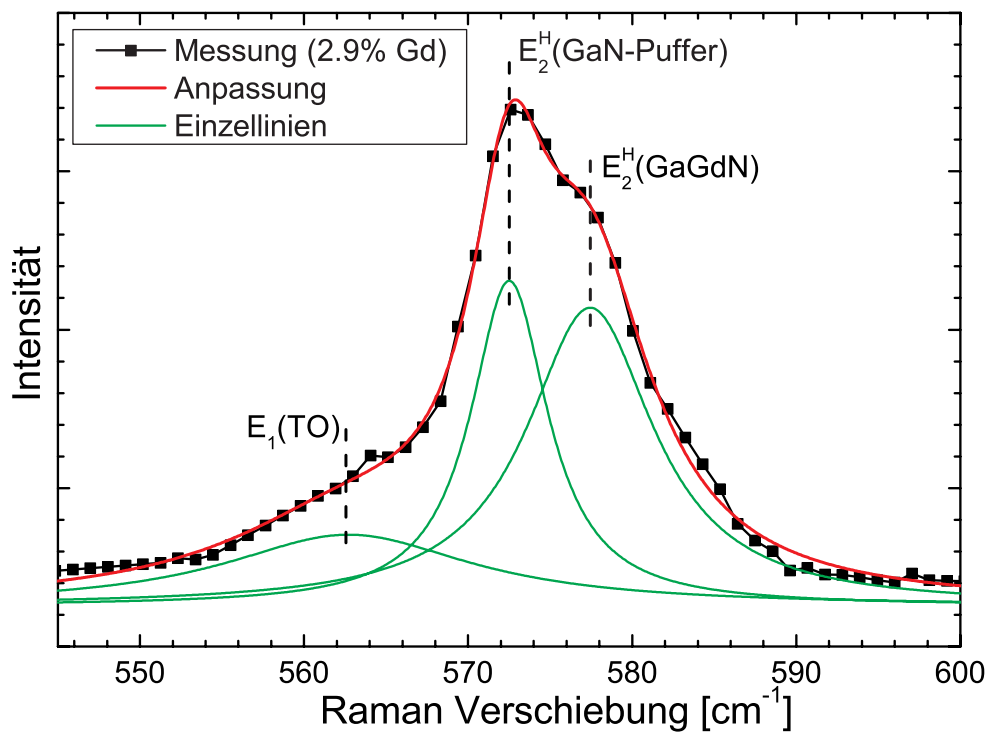

Abbildung 7.9: Raman-Spektrum einer GaGdN-Probe mit 2.9\% Gd-Gehalt nahe der E $_{2}$-Mode. Es ist eine Schulter an der hochenergetischen Flanke erkennbar, die der GaGdN-Schicht zugeordnet wird, während der niederenergetische Anteil dem GaN-Puffer zugeordnet wird.

Die $\mathrm{E}_{2}$-Mode weist eindeutig zwei Anteile auf, die durch eine Anpassung mit LorentzKurven problemlos getrennt werden können. Das Signal bei $572.5 \mathrm{~cm}^{-1}$ wird dabei der GaN-Pufferschicht zugeordnet, eine Referenzprobe ohne GaGdN-Schicht zeigte ein einzelnes Signal an eben dieser Position. Damit stammt das Signal bei $577.4 \mathrm{~cm}^{-1}$ von der GaGdN-Schicht. Ein drittes schwaches Signal bei etwa $563 \mathrm{~cm}^{-1}$ kann der $\mathrm{E}_{1}$ (TO)-Mode einer oder beider Schichten zugeordnet werden, die in der verwendeten Geometrie eigentlich verboten ist, durch den Öffnungswinkel der Detektionsoptik aber schwach erlaubt wird. Die Linienposition der GaN-Pufferschicht zeigt bereits eine deutliche Blauverschiebung gegenüber dem Wert für Volumenproben von $569 \mathrm{~cm}^{-1}$. Dies ist für Schichten auf 
Saphir-Substrat auch so zu erwarten. Die GaGdN-Linie ist dagegen noch einmal deutlich stärker blauverschoben. Die Linienposition kann nicht durch lokale Moden des Gd erklärt werden, da ein schwereres Atom die reduzierte Masse des Metall-Stickstoff-Dimers erhöht und damit nach $\omega \propto \sqrt{c / \mu}$ die Phononenfrequenz erniedrigt. Es kommt für die Linienposition also nur eine extrem blauverschobene $\mathrm{E}_{2}$-Mode des verdünnten GaGdN in Frage. Benutzt man die entsprechenden Konstanten von GaN nach Davydov et al. [85], so kann die Verspannung $\sigma_{x x}$ nach Gleichung 4.17 quantifiziert werden:

$$
\sigma_{x x}=\frac{\Delta \omega_{E_{2}^{H}}}{K_{E_{2}^{H}}}=\frac{4.9 \mathrm{~cm}^{-1}}{2.7 \mathrm{~cm}^{-1} / \mathrm{GPa}} \approx 1.8 \mathrm{GPa}
$$

Auf Grund der bekannten Abhängigkeit der DBX-Linienposition von der Verspannung kann daraus dann eine erwartete Linienposition berechnet werden:

$$
E(\mathrm{DBX})=E_{0}(\mathrm{DBX})+K_{P L} \sigma_{x x}=3.469 \mathrm{eV}+20 \mathrm{meV} / \mathrm{GPa} \cdot 1.8 \mathrm{GPa}=3.505 \mathrm{eV}
$$

Dabei wurde für $E_{0}(\mathrm{DBX})$ der Wert der am niedrigsten dotierten Probe gewählt. Dieses Ergebnis ist in Abbildung 7.8b) als roter Datenpunkt markiert. Der Punkt passt tatsächlich zu der Trendgerade aus den niedrig dotierten Proben. Dies bestätigt Verspannungen als Ursache der Blauverschiebung der DBX-Linie bei kleinen Gd-Konzentrationen. Um allerdings die gemessene DBX-Linie der hoch dotierten Schicht zu erklären, muss durch einen unabhängigen Effekt die Bandlücke um etwa 21 meV kleiner geworden sein. Diese Annahme ist nicht einmal abwegig, da immerhin 2.9\% der Ga-Atome durch Gd ersetzt wurden und somit eigentlich ein ternärer Halbleiter vorliegt. Zum Vergleich, die Bandlücke von $\mathrm{Ga}_{0} .971 \mathrm{In} 0.029 \mathrm{~N}$ liegt etwa $115 \mathrm{meV}$ unter der von GaN [179]. Leider existieren keine zuverlässigen Daten in der Literatur über diesen Effekt von GaGdN. Einzig Teraguchi et al. [180] beobachten eine Rotverschiebung der Bandkantenlumineszenz bei Raumtemperatur, allerdings ohne Analyse der Verspannung, die wie gerade gezeigt wurde von entscheidender Bedeutung ist. Reines GdN als Referenzsubstanz kristallisiert nicht in der Wurtzit-Struktur sondern in der Kochsalz-Struktur. Es besitzt dann eine Bandlücke von etwa $0.8 \mathrm{eV}$ [181] und wird im ferromagnetischen Zustand unterhalb $\mathrm{T}_{c}$ halbmetallisch [182]. Theoretische Berechnungen für GdN in der Zinkblende-Struktur (die nahezu identisch mit der Wurtzit-Struktur ist) ergeben eine Bandlücke von etwa $1 \mathrm{eV}$ [15]. Auch wenn diese theoretischen Berechnungen die Bandlücke normalerweise unterschätzen, liegt die von $\mathrm{GdN}$ deutlich unter der von GaN, die in vergleichbaren Rechnungen um $2 \mathrm{eV}$ liegt. Dieser Trend erklärt also sehr gut die experimentellen Daten in Abbildung 7.8b). Da er in erster Näherung linear mit der Gd-Konzentration skaliert, spielt er für die niedriger dotierten Proben keine Rolle.

Die starken Verspannungen durch die Gd-Atome könnten indirekt auch eine Abnahme der zur YL beitragenden Ga-Vakanzen verursachen. Schon während des Wachstums werden sich die bei diesen Temperaturen relativ gut beweglichen Vakanzen in der Nähe der Gd-Atome anlagern, da durch die Vakanzen eine teilweise Relaxation der kompressiven Verspannung möglich ist. Durch diese Relaxation wird die elektronische Struktur der Vakanz so weit verändert, dass sie nicht mehr als Rekombinationspfad für die gelbe Lumineszenz dienen kann. Bei Dotierungen im Prozentbereich müssen sich die Vakanzen 
im Durchschnitt nur eine Gitterstelle bewegen, um als direkter Nachbar auf dem GaUntergitter zu einem Gd-Atom zu liegen. Dadurch könnte das komplette Fehlen der YL verursacht werden.

\section{Zusammenfassung}

In diesem Kapitel wurde gezeigt, dass in GaGdN keine Gd-spezifischen Lumineszenzen beobachtbar waren. Dies kann auf Grund der isoelektronischen Eigenschaften von $\mathrm{Gd}_{\mathrm{Ga}}$ und der hohen Energie der intra-4f-Lumineszenzen verstanden werden. Allerdings wurde mit steigendem Gd-Einbau eine zunehmende kompressive Verspannung der GaN-Matrix detektiert. In einer hochdotierten Probe schließlich konnte eine Verkleinerung der Bandlücke von GaGdN nachgewiesen werden. 


\section{Zusammenfassung und Ausblick}

Im Rahmen dieser Arbeit wurden verschiedene verdünnte magnetische Halbleiter (DMS) auf GaN-Basis strukturell und optisch charakterisiert. Schwerpunktmäßig wurde dabei GaMnN untersucht, speziell die Auswirkungen der Wachstumsbedingungen auf die optischen Eigenschaften. Zentrales Werkzeug zur Analyse der Schichten war die Photolumineszenz, für die ein auf die Erfordernisse von GaN angepasster Messplatz aufgebaut wurde.

Am Anfang dieser Untersuchungen stand die strukturelle Charakterisierung, ohne deren Ergebnisse weiterführende Aussagen unmöglich wären. In XRD-Spektren wurde in Proben mit hohem Mn-Gehalt dabei eine zweite Phase detektiert, die eine kubische Symmetrie mit einer Gitterkonstante von $3.90 \AA$ aufweist. Dies passt zur ternären Verbindung $\left(\mathrm{Mn}_{1-x} \mathrm{Ga}_{x}\right) \mathrm{Mn}_{3} \mathrm{~N}_{y}$ mit $x \approx 1 \approx y$, was durch lokale EDX-Messungen bestätigt werden konnte. Für niedrige Wachstumstemperaturen wurde ein Wachstumsfenster im Parameterraum gefunden, in dem GaMnN ohne diese $\mathrm{GaMn}_{3} \mathrm{~N}$-Ausscheidungen hergestellt werden kann. Dieses Fenster liegt bei stickstoffreichen Bedingungen und relativen Mn-Flüssen bis 15\%. Die Mn-Konzentration kann dabei kontrolliert bis 5\% eingestellt werden.

Der Nachteil dieser Wachstumsbedingungen ist eine signifikant verschlechterte Kristallqualität, verglichen mit Proben bei höheren Wachstumstemperaturen. Außerdem wurde in der Anfangsphase $\mathrm{Si}(111)$ als Substrat eingesetzt, das bekannterweise kein optimales Substrat für das GaN-Wachstum darstellt. Die strukturelle Qualität kann zum Einen durch die Breiten der Röntgenreflexe verfolgt werden und zum Anderen durch RamanMessungen. So konnten in allen hoch dotierten GaMnN-Proben Unordnungs-aktivierte Raman-Streuung (DARS) an Stelle der $A_{1}(\mathrm{LO})$-Mode nachgewiesen werden, bei niedrigen Wachstumstemperaturen trat DARS zusätzlich an der $E_{2}^{H}$-Mode auf. Dies zeigt einen Zusammenbruch der kristallinen Fernordnung an, der durch den Mn-Einbau ausgelöst wird. Ein wesentliches Ergebnis ist allerdings die Konstanz der Kristallqualität der GaMnN-Proben innerhalb des Wachstumsfensters. Nach diesen grundlegenden Untersuchungen zum Wachstum wurden dann zur Verbesserung der Materialqualität Proben auf Saphir-Substrat mit MOCVD-gewachsener GaN-Pufferschicht hergestellt. Dadurch wurde eine Reduktion der Breite der rocking curve des (0002)-Reflexes um den Faktor 6 erreicht. Dies ist gleichzeitig wesentlich besser als alle GaN-Referenzproben auf Si-Substrat, was den starken Einfluss der Substratwahl unterstreicht.

Auf der Basis dieser Ergebnisse können die Lumineszenz-Spektren der GaMnN-Proben analysiert werden. So korrelieren die PL-Messungen klar mit den XRD-Messungen in Bezug auf die Kristallqualität. An Hand des Vorhandenseins und der Linienbreite der exzitonischen Übergänge kann dies für den gesamten Parameterraum nachvollzogen werden. Eine Einschränkung hierbei ist der Effekt von Mn-Konzentrationen ab etwa 1\%, die Lumineszenz nahezu vollständig zu unterdrücken. Als ideal für optische Untersuchungen stellen 
sich damit Proben heraus, die bei hohen Temperaturen mit niedrigem bis mittlerem MnGehalt hergestellt wurden. In diesem Fall kann auf Saphir-Substrat die hohe Qualität des MOCVD-gewachsenen GaN-Puffers auch in der GaMnN-Schicht gehalten werden. Diese hohe Qualität ermöglicht beispielsweise den Nachweis der Eigenschaft als tiefer Akzeptor von $\mathrm{Mn}$ in GaN. Außerdem zeigen magneto-optische Untersuchungen der freien Exzitonen in GaMnN, dass eine schwache Kopplung der lokalen Mn-Spins mit den Ladungsträgern der GaN-Matrix existiert. Dies ist die Grundvoraussetzung für eine technische Anwendung der verdünnten magnetischen Halbleiter beispielsweise als Spininjektoren.

Ein weiteres hochinteressantes Gebiet eröffnete sich durch den Fund und die Identifikation des intra-3d-Übergangs ${ }^{5} E \rightarrow{ }^{5} T_{2}$ von $\mathrm{Mn}^{3+}$ bei $1.41-1.42 \mathrm{eV}$ in den PL-Spektren von GaMnN. Dieser wurde umfassend charakterisiert und mit entsprechenden Daten für die Absorption, die aus der Literatur bekannt sind, verglichen. Es zeigte sich auch an dieser Stelle die überlegene optische Qualität der Proben auf Saphir-Substrat. Die NullPhononen-Linie (ZPL) konnte in drei einzelne Linien aufgelöst werden, wobei ZPL 1 und 2 aus einer Aufspaltung durch die Spin-Bahn-Wechselwirkung resultieren, während ZPL 3 durch die trigonale Symmetrie des Wurtzit bzw. der Verspannung verursacht wird. Letzteres konnte durch Vergleich verschieden verspannter Proben auch auf unterschiedlichen Substraten direkt experimentell beobachtet werden. Die Temperaturabhängigkeit schließlich zeigte, dass die Aufspaltung im Grundzustand des Übergangs auftritt und nicht im angeregten Zustand. Auf Grund dieser Analysen und der Magnetfeld-abhängigen Messungen des Übergangs kann klar das Modell des dynamischen Jahn-Teller-Effekts von Marcet et al. [37] bestätigt werden, im Gegensatz zum Modell von Wolos et al. [35], die einen statischen Jahn-Teller-Effekt vorschlagen. Die Absorptionsmessungen weisen gleichzeitig den Ladungszustand $\mathrm{Mn}^{3+}$ in den vorliegenden Proben direkt nach. Ein solcher Zustand mit teilweise besetzten $d$-Zuständen ist die Voraussetzung für die magnetische Kopplung der Mn-Atome nach dem Doppelaustausch, eine der diskutierten Theorien für GaN-basierte DMS. Auf diese Art von Kopplung deutet auch das Verhalten des intra-3d-Übergangs bei hohen Mn-Konzentrationen hin, der sich erst verbreitert und dann nicht mehr nachweisbar ist.

Zusätzlich zu den GaMnN-Schichten wurden weitere GaN-basierte DMS optisch charakterisiert. GaCrN zeigt dabei unter den Wachstumsbedingungen, die für die MOCVD gewählt wurden, eine starke Lumineszenz im Blauen (BL). Diese BL zeigt ein signifikant anderes Verhalten als die in der Literatur bekannte BL in GaCrN, GaMnN oder undotiertem GaN. Es kann einem intrinsischen Defekt zugeordnet werden, der durch die niedrige Wachstumstemperatur verursacht wird. Vergleichbar dem Mn zeigt auch $\mathrm{Cr}$ in GaN einen intra-3d-Übergang. Dieser dem $\mathrm{Cr}^{4+}$ zugeordnete Übergang kann bei $1.19 \mathrm{eV}$ detektiert werden und der mehr als 10 Jahre alte Streit um die Ursache dieser Linie geklärt werden. GaGdN zeigt keine Gd-spezifischen Lumineszenzen, allerdings eine Verschiebung der bandkantennahen Lumineszenz mit der Gd-Konzentration. Dies kann unter Zuhilfenahme von Raman-Messungen nachgewiesen werden, wodurch kompressive Verspannungen als Ursache identifiziert werden. Außerdem ist dadurch bei Dotierung im Prozentbereich eine Verkleinerung der Bandlücke von GaGdN nachweisbar. 
Ausblick Sieben Jahre nach der Veröffentlichung von Dietl et al. [9], die das Materialsystem GaMnN in den Fokus stellte, lässt das Interesse langsam nach. Dies äußert sich beispielsweise in der Zahl der Publikationen zum Thema, die nach mehreren Jahren auf hohem Niveau langsam zurückgeht. Das liegt zum Einen daran, dass dieses Material nicht leicht in zufriedenstellender Qualität herzustellen ist und zum Anderen daran, dass die magnetischen Eigenschaften nach wie vor unbefriedigend sind. Auch einige theoretische Überlegungen deuten darauf hin, dass Ferromagnetismus bei Raumtemperatur nicht möglich ist, oder erst bei Konzentrationen um 20\% [48], was weit außerhalb der momentanen experimentellen Möglichkeiten liegt. Allerdings zeigen auch die im Rahmen dieser Arbeit untersuchten Proben Anzeichen einer ferromagnetischen Kopplung und zum Teil sogar Raumtemperatur-Ferromagnetismus. Ein wichtiger Ansatz hierbei ist möglicherweise die Theorie von Fukushima et al. [49], die basierend auf der vorhergesagten Entmischung von GaMnN mit Hilfe eines geeigneten Wachstumsprozesses eine deutliche Erhöhung der Curie-Temperatur vorhersagt. Bereits in den optischen Spektren fanden sich Hinweise auf Unordnungs-induzierte Effekte und es konnte sogar mittels Raster-EDX-Aufnahmen im TEM direkt eine Entmischung des Mn in einigen Proben nachgewiesen werden. Durch diese lokal hohen Konzentrationen könnte dann eine wesentliche Verstärkung der magnetischen Kopplung erreicht werden. Wenn es gelingt, an solchen Proben magneto-optische Messungen an der Bandkante durchzuführen, ist hier möglicherweise ein deutlich stärkerer Effekt als in den bisherigen Proben nachweisbar.

Sollte es sich herausstellen, dass GaMnN letztendlich nicht für Spininjektion geeignet ist, steht mit GaGdN bereits ein potentieller Kandidat in den Startlöchern, der bereits bei niedrigen Konzentrationen starken Ferromagnetismus bis oberhalb Raumtemperatur zeigt. Und auch bei hohen Konzentrationen sind die optischen Eigenschaften von GaGdN noch deutlich besser als etwa bei GaMnN oder GaCrN. Eine logische Konsequenz wären daher magneto-optische Messungen an an GaGdN. Damit könnten die magnetischen Eigenschaften besser verstanden werden, denn nach wie vor ist nicht geklärt, wovon die starken magnetischen Momente von über $1000 \mu_{B} / G d$-Atom bei niedrigen GdKonzentrationen ausgelöst werden. Eine Polarisation der umgebenden Matrix sollte sich in einer starken Kopplung mit den Ladungsträgern über einen $s$ - $f$-Austausch ausdrücken. Vielleicht gelingt daher mit diesem Material sogar die Konstruktion einer Spin-LED, also einer Leuchtdiode mit Gadolinium in der aktiven Schicht, die dann spin-polarisiertes Licht emittiert. Dies wäre dann der direkte Beweis einer erfolgreichen Spininjektion. Damit wäre der erste wichtige Schritt für die Anwendung getan und zwar die Realisierung einer spinpolarisierten DMS-Schicht bei Raumtemperatur. Der nächste Schritt, die Realisierung von Spintransport, scheint durch die erwiesenermaßen hohe Spin-Kohärenzlänge in GaN unter Kontrolle zu sein. Die Spinmanipulation (beispielsweise über den Rashba-Effekt) und Detektion sind noch Gegenstand intensiver aktueller Forschung. 


\section{Anhang A: Korrektur der temperaturabhängigen Messungen}

Bei den temperaturabhängigen PL-Messungen im Infraroten traten die in Abbildung A.1 dargestellten Probleme auf.

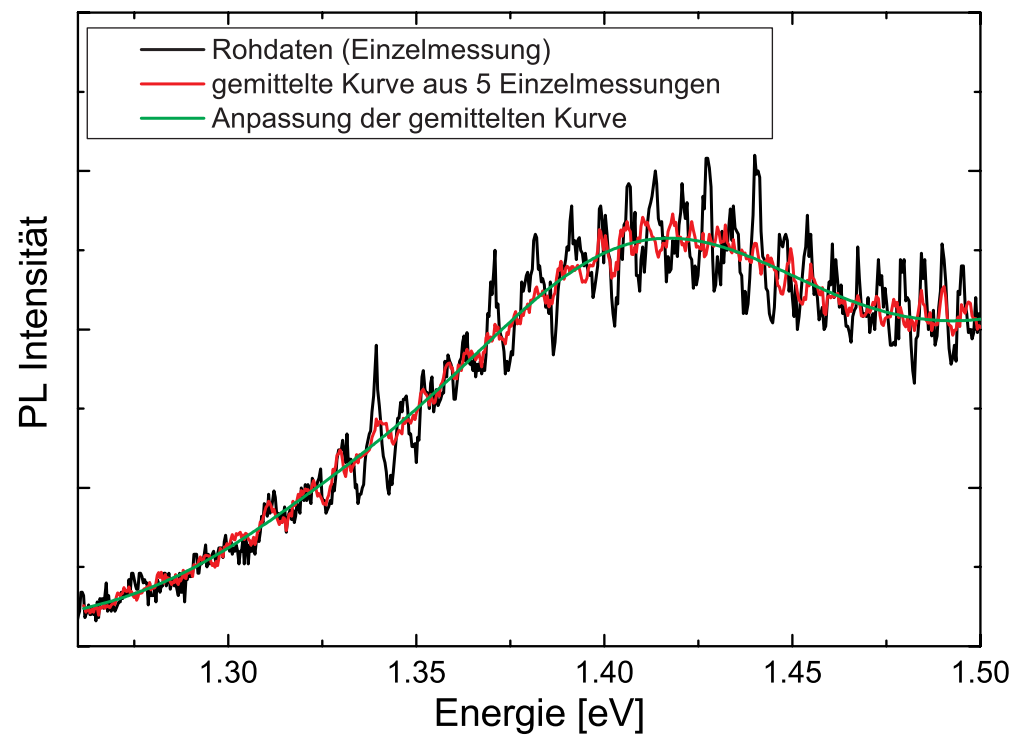

Abbildung A.1: Verfahren zur Optimierung der Datenqualität bei temperaturabhängigen Messungen:

Die Rohdaten werden gemittelt und dann die Hochtemperaturmessung mittels Gauss-Funktionen angepasst.

Bei der Messung kam es zu Interferenzeffekten, die eine reproduzierbare, aber unregelmäßige Oszillation der Messkurve hervorriefen. Wurde das Gitter verdreht, veränderte sich auch dieses Messartefakt. Diese Änderung wurde ausgenutzt, um im ersten Schritt durch 5 Einzelmessungen mit jeweils leicht verschobenem Gitter durch Mittelung eine höhere Datenqualtität zu erzielen. Es zeigt sich allerdings, dass diese immer noch nicht ausreichte, um Analysen an der Lumineszenz bei $1.4 \mathrm{eV}$ durchführen zu können. Nun wurde ausgenutzt, dass das Artefakt unabhängig von der Temperatur in jeder Messung auftrat. Bei hohen Temperaturen ist die Lumineszenz in diesem Spektralbereich eine sehr glatte Funktion, es treten keine scharfen Linien auf. Daher wurde dort die Messkurve bei $295 \mathrm{~K}$ durch mehrere gaussförmige Signale angepasst, bis sich eine sehr gute Übereinstimmung ergab, wie in Abbildung A.1 zu sehen ist. Schließlich wurde der Quotient aus Anpassung und Messung gebildet und alle Spektren bei anderen Temperaturen wurden punktweise mit diesem Quotienten multipliziert:

$$
y_{\text {korrigiert }}(T)=\frac{y_{\text {angepasst }}(295 K)}{y_{\text {gemessen }}(295 K)} \times y_{\text {gemessen }}(T)
$$

y steht dabei für die PL-Intensität. Die Anpassung wurde mit der Messung bei $250 \mathrm{~K}$ wiederholt, um auch die Messung bei $295 \mathrm{~K}$ mit einer vergleichbaren Prozedur korrigieren zu können. 
Das Ergebnis dieses Verfahrens für die oben gezeigte Messung ist in Abbildung $6.9 \mathrm{zu}$ sehen. Zwar wird durch dieses Verfahren das Rauschen um den Faktor $\sqrt{2}$ erhöht durch Division zweier Messgrößen (Gleichung A.1) und ein zusätzlicher Fehler durch die Anpassung erzeugt, andererseits zeigt der Vergleich mit Tieftemperaturmessungen im StandardPL-Aufbau, dass keine zusätzlichen Artefakte auftreten. Daher wurde dieses Verfahren für alle Messungen an diesem Aufbau im Infraroten eingesetzt. 


\section{Anhang B: Überblick über die GaMnN-Proben}

In Tabelle B.1 sind die wichtigsten Wachstumsparameter der in dieser Dissertation namentlich angesprochenen GaMnN-Proben zusammengefasst, zusammen mit dem MnGehalt, wie er mittels EDX bzw. SIMS ermittelt wurde. Die Parameter der Stickstoffquelle für die Jxxxx-Proben sind $\Phi_{N_{2}}=1 \mathrm{sccm}$ und $P=450 \mathrm{~W}$, für die Gxxxx-Proben gilt $\Phi_{N_{2}}=0.5 \mathrm{sccm}$ und $P=300 \mathrm{~W}$.

\begin{tabular}{|c||c|c|c|c|c|}
\hline Probenname & Substrat & $\begin{array}{c}\text { Wachstums- } \\
\text { temperatur }\end{array}$ & Mn-Gehalt & $\begin{array}{c}\text { Ga-Fluss } \\
{\left[10^{-8} \text { mbar }\right]}\end{array}$ & $\begin{array}{c}\text { Mn-Fluss } \\
{\left[10^{-8} \text { mbar }\right]}\end{array}$ \\
\hline \hline J0027 & $\mathrm{Si}(111)$ & $760^{\circ} \mathrm{C}$ & $0.5 \%$ & 10 & 0.5 \\
$\mathrm{~J} 0028$ & $\mathrm{Si}(111)$ & $760^{\circ} \mathrm{C}$ & $0.5 \%$ & 10 & 1 \\
$\mathrm{~J} 0080$ & $\mathrm{Si}(111)$ & $730^{\circ} \mathrm{C}$ & $4.5 \%$ & 2.45 & 1.05 \\
$\mathrm{~J} 0084$ & $\mathrm{Si}(111)$ & $730^{\circ} \mathrm{C}$ & $0.3 \%$ & 2.625 & 0.875 \\
$\mathrm{~J} 0087$ & $\mathrm{Si}(111)$ & $650^{\circ} \mathrm{C}$ & $32.6 \%$ & 0.6 & 0.4 \\
$\mathrm{~J} 0088$ & $\mathrm{Si}(111)$ & $650^{\circ} \mathrm{C}$ & $11.3 \%$ & 0.75 & 0.25 \\
$\mathrm{~J} 0091$ & $\mathrm{Si}(111)$ & $650^{\circ} \mathrm{C}$ & $2.6 \%$ & 0.85 & 0.15 \\
$\mathrm{~J} 0092$ & $\mathrm{Si}(111)$ & $650^{\circ} \mathrm{C}$ & $4.1 \%$ & 0.85 & 0.15 \\
$\mathrm{~J} 0093$ & $\mathrm{Si}(111)$ & $650^{\circ} \mathrm{C}$ & $2.7 \%$ & 0.9 & 0.1 \\
$\mathrm{~J} 0094$ & $\mathrm{Si}(111)$ & $650^{\circ} \mathrm{C}$ & $9.2 \%$ & 0.8 & 0.2 \\
$\mathrm{~J} 0095$ & $\mathrm{Si}(111)$ & $650^{\circ} \mathrm{C}$ & $1.4 \%$ & 0.95 & 0.05 \\
$\mathrm{~J} 0117$ & $\mathrm{Si}(111)$ & $650^{\circ} \mathrm{C}$ & $5.0 \%$ & 1.7 & 0.3 \\
$\mathrm{~J} 0121$ & $\mathrm{Si}(111)$ & $650^{\circ} \mathrm{C}$ & $14.6 \%$ & 4.25 & 0.75 \\
$\mathrm{~J} 0125$ & $\mathrm{Si}(111)$ & $650^{\circ} \mathrm{C}$ & $12.3 \%$ & 2.55 & 0.45 \\
$\mathrm{~J} 0130$ & $\mathrm{Si}(111)$ & $650^{\circ} \mathrm{C}$ & $1.7 \%$ & 0.85 & 0.15 \\
$\mathrm{~J} 0135$ & $\mathrm{Si}(111)$ & $650^{\circ} \mathrm{C}$ & & 5.19 & 0.106 \\
$\mathrm{~J} 0136$ & $\mathrm{Si}(111)$ & $650^{\circ} \mathrm{C}$ & & 3.92 & 0.08 \\
$\mathrm{~J} 0137$ & $\mathrm{Si}(111)$ & $650^{\circ} \mathrm{C}$ & $1.0 \%$ & 2.88 & 0.12 \\
$\mathrm{~J} 0138$ & $\mathrm{Si}(111)$ & $650^{\circ} \mathrm{C}$ & $2.6 \%$ & 1.92 & 0.08 \\
$\mathrm{~J} 0146$ & $\mathrm{Si}(111)$ & $650^{\circ} \mathrm{C}$ & & 2 & $\approx 0.03$ \\
$\mathrm{~J} 0148$ & $\mathrm{Si}(111)$ & $650^{\circ} \mathrm{C}$ & & 2 & $\approx 0.012$ \\
G0103 & $\mathrm{Saphir}$ & $775^{\circ} \mathrm{C}$ & $0.5 \%$ & 16.4255 & 0.8645 \\
G0105 & $\mathrm{Saphir}$ & $775^{\circ} \mathrm{C}$ & & 13.8985 & 0.7315 \\
G0117 & $\mathrm{Saphir}$ & $775^{\circ} \mathrm{C}$ & $0.07 \%$ & 17.1171 & 0.1729 \\
$\mathrm{G} 0119$ & $\mathrm{Saphir}$ & $775^{\circ} \mathrm{C}$ & $0.015 \%$ & 17.2381 & 0.0536 \\
$\mathrm{G} 0121$ & $\mathrm{Saphir}$ & $775^{\circ} \mathrm{C}$ & $2.0 \%$ & 15.561 & 1.729 \\
$\mathrm{G} 0131$ & $\mathrm{Saphir}$ & $775^{\circ} \mathrm{C}$ & $0.003 \%$ & 17.2848 & 0.0052 \\
$\mathrm{G} 0142$ & $\mathrm{Saphir}$ & $775^{\circ} \mathrm{C}$ & $3.0 \%$ & 14.364 & 1.596 \\
\hline
\end{tabular}

Tabelle B.1: Wichtige Parameter aller analysierten GaMnN-Proben. 


\section{Abbildungsverzeichnis}

2.1 Zinkblende und Wurtzit: Kristallstruktur. . . . . . . . . . . . . . 4

2.2 Ausgleich der Gitterfehlanpassung durch Verformung. . . . . . . . . . . . 6

2.3 Erste Brillouin-Zone eines Wurtzit-Kristalls . . . . . . . . . . . . 7

2.4 Elektronische Bandstruktur von GaN . . . . . . . . . . . . . . 7

2.5 Aufspaltung der Valenzbänder in GaN durch Spin-Bahn-Wechselwirkung und das Kristallfeld . . . . . . . . . . . . . . . . . . . . . 8

2.6 Ionen bei oktaedrischer und tetraedrischer Koordination . . . . . . . . 11

2.7 Aufspaltung der $d$-Orbitale im oktaedrischen Kristallfeld . . . . . . . . . 13

$2.8 d$-Orbitale . . . . . . . . . . . . . . . . . . . . . . . . . . . 14

2.9 Umladungsniveaus von $\mathrm{Mn}$ in verschiedenen III-V-Halbleitern . . . . . . 15

2.10 Aufspaltung des Grundzustandes eines $d^{4}$-Elektronensystems im Tetraederfeld . . . . . . . . . . . . . . . . . . . . . . . . 16

2.11 Tanabe-Sugano Diagramm eines $d^{4}$-Systems im tetraedrischen Kristallfeld . 17

2.12 Tetragonale und trigonale Verzerrung eines Tetraeders . . . . . . . . . . . 18

2.13 Potentielle Energie eines T-Zustandes gekoppelt mit einer E-Mode aus [35] 18

2.14 Konfigurations-Koordinaten-Diagramm für zwei elektronische Zustände in harmonischer Näherung . . . . . . . . . . . . . . . . 21

2.15 Energieniveaus von $\mathrm{Mn}^{3+}$ in GaN nach [37] . . . . . . . . . . . . . 22

2.16 Struktur eines verdünnten magnetischen Halbleiters im Vergleich zu intrinsischem Halbleiter und klassischem Ferromagneten wie etwa Eisen. . . . . . 23

2.17 Superaustausch . . . . . . . . . . . . . . . . . . . . . 24

2.18 Doppelaustausch . . . . . . . . . . . . . . . . . 25

2.19 p-d-Austausch . . . . . . . . . . . . . . . . . . . . 26

2.20 Von Dietl et al. [9] berechnete Curie-Temperaturen für verschiedene Halbleiter 27

2.21 Schematisches Modell der elektronischen Zustände von Mn in GaAs und $\mathrm{GaN} \ldots \ldots \ldots \ldots \ldots \ldots \ldots \ldots$

2.22 Stabilität der ferromagnetischen gegenüber der antiferromagnetischen (Spin-Glas-)Phase bei GaN . . . . . . . . . . . . . . . . . . . . . . 29

3.1 Aufbau einer MBE-UHV-Kammer . . . . . . . . . . . . . . . . . . . 31

3.2 Oberflächenprozesse beim MBE-Wachstum . . . . . . . . . . . . . 32

3.3 Darstellung des Parameter-Raums als ,spider web“. . . . . . . . . . . . 34

3.4 Aufbau der GaMnN- und GaGdN-Proben . . . . . . . . . . . . . 36 
4.1 Verschiedene Rekombinationskanäle für angeregte Elektronen und Löcher in Halbleitern. . . . . . . . . . . . . . . . . . . . . . . . . . . 37

4.2 Absorption eines Photons und Relaxation der entstehenden Elektronen und Löcher durch Phononen. . . . . . . . . . . . . . . . . . . 38

4.3 Abhängigkeit der Exzitonenenergien von der Deformation in c-Richtung . . 42

4.4 Konfigurations-Koordinaten Diagramm für die YL in GaN . . . . . . . . 44

4.5 Schematischer Aufbau des PL-Systems. . . . . . . . . . . . . . . 45

4.6 Phononenmoden in Wurtzit-Kristallen . . . . . . . . . . . . . . . . . . 49

4.7 Phononen-Dispersionsrelation von $\mathrm{GaN}$. . . . . . . . . . . . . . 50

4.8 Schematischer Aufbau des verwendeten TOFSIMS-Systems. . . . . . . . 56

4.9 Schematischer Aufbau des XRD-Gerätes. . . . . . . . . . . . . . . 58

5.1 XRD-Messung an einer Probe mit hohem Mn-Angebot . . . . . . . . . 60

5.2 Struktur von $\left(\mathrm{Mn}_{1-x} \mathrm{Ga}_{x}\right) \mathrm{Mn}_{3} \mathrm{~N} \ldots \ldots \ldots \ldots$

5.3 Hochauflösende XRD-Messung an Probe J0121 mit 14.6 \% Mn-Gehalt . . . 63

5.4 EDX-Analyse einer Ausscheidung in Probe J0121 . . . . . . . . . . . . 64

5.5 XRD-Spektren von Proben mit verändertem Mn-Angebot und verändertem Gesamtmetall-Angebot . . . . . . . . . . . . . . . . . . . 65

5.6 Wachstumsfenster im Parameter-Raum zum Herstellen von reinem GaMnN 66

5.7 FWHM der Röntgenmessungen am (0002)-Reflex von GaMnN bei unterschiedlicher Wachstumstemperatur . . . . . . . . . . . . . 67

5.8 Raman-Spektren von GaMnN in Abhängigkeit von der Wachstumstemperatur . . . . . . . . . . . . . . . . . . . . . 68

5.9 FWHM der Röntgenmessungen am (0002)-Reflex von GaMnN ohne Ausscheidungen mit unterschiedlichem Mn-Gehalt . . . . . . . . . . 70

5.10 Raman-Spektren von GaMnN in Abhängigkeit von den Wachstumsbedingungen . . . . . . . . . . . . . . . . . . 71

5.11 Vergleich der rocking curves am (0002)-Reflex von Proben auf unterschiedlichen Substraten . . . . . . . . . . . . . . . . . . 72

5.12 RBS-Spektrum von Probe J0117 mit Simulation . . . . . . . . . . . . . . 74

5.13 RBS-Spektrum von Probe J0117 in channeling-Geometrie im Vergleich zur random-Messung . . . . . . . . . . . . . . . 75

6.1 PL-Spektren von zwei GaN-Referenzproben . . . . . . . . . . . . 78

6.2 PL-Spektren von GaMnN bei verschiedenen Wachstumstemperaturen . . . 79

6.3 PL-Spektren einer Probe mit „Raman-Linien“ nahe der Bandlücke bei verschiedenen Temperaturen . . . . . . . . . . . . . . . . . . . 80

6.4 PL-Spektren bei Veränderung des Mn-Angebotes . . . . . . . . . . . 82

6.5 Vergleich von GaN-Referenzproben und GaMnN auf Si(111) und Saphir . . 83

6.6 GaMnN mit unterschiedlichem Mn-Gehalt . . . . . . . . . . . . . 84

6.7 Leistungsabhängigkeit der exzitonischen Übergänge in GaMnN . . . . . . . 86

6.8 PL im Infraroten an Probe J0027 und einer GaN-Referenzprobe . . . . . . 87

6.9 Temperaturabhängigkeit der Lumineszenz bei $1.4 \mathrm{eV}$ von Probe J0080 . . . 88 
6.10 Direkter Vergleich einer Absorptionsmessung von Wolos et al. [35] mit der PL-Messung an Probe J0027 . . . . . . . . . . . . . . . . . . . . . 90

6.11 Verhalten der Lumineszenz bei $1.42 \mathrm{eV}$ bei Erhöhung des Mn-Angebots . . 92

6.12 Verhalten der Lumineszenz bei $1.42 \mathrm{eV}$ bei Erhöhung des Ga-Angebots . . 92

6.13 Vergleich von zwei Proben bei hoher Wachstumstemperatur auf Si-Substrat (J0027) und Saphir-Substrat (G0103) . . . . . . . . . . . . . . . 94

6.14 Verschiebung der exzitonischen Linien und intra-3d-Linien in Abhängigkeit von der DBX-Linienposition . . . . . . . . . . . . . . . . 96

6.15 PL-Spektren im IR von hochqualitativem GaMnN mit verschiedenem MnGehalt . . . . . . . . . . . . . . . . . 97

6.16 Temperaturabhängigkeit der Lumineszenz bei $1.4 \mathrm{eV}$ von Probe G0103 . . 98

6.17 Auger-artiger Prozess zur Anregung von intra-atomarer Lumineszenz . . . 100

6.18 Faraday- und Voigt-Geometrie in der Magneto-Optik. . . . . . . . . . . . . 101

6.19 PL-Spektren nahe der Bandkante für zwei Proben mit unterschiedlichen Mn-Gehalt in Abhängigkeit vom Magnetfeld . . . . . . . . . . . . . . . . . 102

6.20 Position der Signale von FXA und $\operatorname{FXA}(n=2)$ in Abhängigkeit vom Magnetfeld . . . . . . . . . . . . . . . . . 103

6.21 Absorptionsspektren von GaMnN mit unterschiedlicher Mn-Konzentration. 105

6.22 Absorptionsspektrum bei $1.4 \mathrm{eV}$ von Probe G0103 in Abhängigkeit vom Magnetfeld ....................... . . 107

6.23 PL-Spektrum bei $1.4 \mathrm{eV}$ von Probe G0131 in Abhängigkeit vom Magnetfeld109

6.24 PL-Spektren bei $1.4 \mathrm{eV}$ von Probe G0131 in Abhängigkeit von der Temperatur bei einem B-Feld von 11 Tesla. . . . . . . . . . . . . . . . . 110

6.25 PL-Übergänge nach dem Modell von Marcet et al. (vgl. Abbildung 2.15). . 111

6.26 PL-Spektren bei $1.4 \mathrm{eV}$ von Probe G0117 in Abhängigkeit vom Magnetfeld in Voigt-Konfiguration, gemessen bei 1.8 K. . . . . . . . . . . . . . . . . 111

7.1 Aufbau der GaCrN-Proben . . . . . . . . . . . . . . . . . . . . . 114

7.2 PL-Spektren von GaCrN-Proben mit unterschiedlichen Wachstumstemperaturen . . . . . . . . . . . . . . . . . . . . . . . . 114

7.3 PL-Spektren einiger bei $950^{\circ} \mathrm{C}$ gewachsenen GaCrN-Proben mit unterschiedlichen Cr-Gehalt . . . . . . . . . . . . . . . . . . 116

7.4 Detail-Spektrum der Bandkante und der hochenergetischen Flanke der BL einer repräsentativen Probe . . . . . . . . . . . . . . . . . . 117

7.5 Temperaturabhängige PL-Spektren der BL . . . . . . . . . . . . . . . 118

7.6 PL-Spektren im Infraroten einer bei $950^{\circ} \mathrm{C}$ gewachsenen $\mathrm{GaCrN}$-Probe und einer GaN-Referenz . . . . . . . . . . . . . . . . . . . . . . . . . . 119

7.7 PL-Spektren im Infraroten der bei $950^{\circ} \mathrm{C}$ gewachsenen $\mathrm{GaCrN}$-Proben mit unterschiedlichem Cr-Gehalt . . . . . . . . . . . . . . . 121

7.8 Auf das DBX-Signal normierte PL-Spektren der GaGdN-Proben mit unterschiedlicher Gd-Konzentration . . . . . . . . . . . . . . . . . . 123

7.9 Raman-Spektrum einer GaGdN-Probe mit 2.9\% Gd-Gehalt nahe der $\mathrm{E}_{2^{-}}$ Mode . . . . . . . . . . . . . . . . . . . . . . . . 124 
A.1 Verfahren zur Optimierung der Datenqualität bei temperaturabhängigen Messungen . . . . . . . . . . . . . . . . . . . . . . . . . 130 


\section{Tabellenverzeichnis}

2.1 Wichtige physikalische Kenngrößen von GaN . . . . . . . . . . . . . 3

3.1 Wichtige Eigenschaften von GaN im Vergleich zu verschiedenen Substraten 35

4.1 Wichtige Lumineszenzlinien und -bänder in GaN . . . . . . . . . . . . . . 41

4.2 Phononenmoden am $\Gamma$-Punkt von Wurtzit-GaN . . . . . . . . . . . . . 49

4.3 Implantationsparameter zur Erzeugung der Referenzproben für SIMSMessungen und daraus mittels TRIM berechnete Eindringtiefe. . . . . . . 55

5.1 Röntgenreflexe in Probe J0087 mit 32.6\% Mn-Gehalt . . . . . . . . . . 60

5.2 Röntgenreflexe von $\mathrm{Mn}_{4} \mathrm{~N}$ und $\mathrm{GaMn}_{3} \mathrm{~N}_{0.5} \ldots \ldots \ldots \ldots$

5.3 Halbwertsbreiten der rocking curves aus Abbildung 5.11. . . . . . . . . 73

5.4 Ergebnisse der RBS-Messungen an drei verdünnten GaMnN-Proben im Vergleich mit anderen Charakterisierungen durch EDX und Dektak. . . . . 75

6.1 Aufgelöste Linien in Abbildung 6.13 . . . . . . . . . . . . . . . . . 95

6.2 Vergleich der Mn-Konzentration aus SIMS-Messungen mit der $\mathrm{Mn}^{3+}$ Konzentration aus Absorptionsmessungen. . . . . . . . . . . . 106

B.1 Wichtige Parameter aller analysierten GaMnN-Proben . . . . . . . . 132 


\section{Literaturverzeichnis}

[1] S. Nakamura, T. Mukai, and M. Senoh. Candela-class high-brightness InGaN/AlGaN double-heterostructure blue-light-emitting diodes. Applied Physics Letters, 64(13):1687-1689, 1994.

[2] S. Nakamura, M. Senoh, N. Iwasa, and S. Nagahama. High-power InGaN singlequantum-well-structure blue and violet light-emitting diodes. Applied Physics Letters, 67(13):1868-1870, 1995.

[3] S. Nakamura, M. Senoh, N. Iwasa, S. Nagahama, T. Yamada, and T. Mukai. Superbright green InGaN single-quantum-well-structure light-emitting diodes. Japanese Journal of Applied Physics Part 2, 34:L1332, 1995.

[4] S. Nakamura, M. Senoh, N. Iwasa, and S. Nagahama. High-brightness InGaN blue, green and yellow light-emitting diodes with quantum-well structures. Japanese Journal of Applied Physics Part 2, 34:L797, 1995.

[5] S. Nakamura, M. Senoh, S. Nagahama, N. Iwasa, T. Yamada, T. Matsushita, H. Kiyoku, and Y. Sugimoto. InGaN-based multi-quantum-well-structure laser diodes. Japanese Journal of Applied Physics Part 2, 35:L74, 1996.

[6] S. Datta and B. Das. Electronic analog of the electro-optic modulator. Applied Physics Letters, 56(7):665-667, 1990.

[7] C. Gould, G. Schmidt, G. Richter, R. Fiederling, P. Grabs, and L. W. Molenkamp. Spin injection into semiconductors using dilute magnetic semiconductors. Applied Surface Science, 190:395-402, 2002.

[8] T. Jungwirth, Jairo Sinova, J. Masek, J. Kucera, and A. H. MacDonald. Theory of ferromagnetic (III,Mn)V semiconductors. Reviews of Modern Physics, 78:809, 2006.

[9] T. Dietl, H. Ohno, F. Matsukura J. Cibert, and D. Ferrand. Zener Model Description of Ferromagnetism in Zinc-Blende Magnetic Semiconductors. Science, 287:1019, 2000 .

[10] S. Dhar, O. Brandt, A. Trampert, L. Däweritz, K. J. Friedland, K. H. Ploog, J. Keller, B. Beschoten, and G. Güntherodt. Origin of high-temperature ferromagnetism in $(\mathrm{Ga}, \mathrm{Mn}) \mathrm{N}$ layers grown on $4 \mathrm{H}-\mathrm{SiC}(0001)$ by reactive molecular-beam epitaxy. Applied Physics Letters, 82:2077, 2003. 
[11] S. Kuwabara, T. Kondo, T. Chikyow, P Ahmet, and H. Munekata. Melecular Beam Epitaxy of Wurtzite GaN-Based magnetic Alloy Semiconductors. Japanese Journal of Applied Physics, 40:L724, 2001.

[12] S. Sonoda, S. Shimizu, T. Sasaki, Y. Yamamoto, and H. Hori. Molecular beam epitaxy of wurtzite (Ga,Mn)N films on sapphire (0001) showing the ferromagnetic behaviour at room temperature. Journal of Crystal Growth, 237:1358, 2002.

[13] K. Sato and H. Katayama-Yoshida. First principles materials design for semiconductor spintronics. Semiconductor Science and Technology, 17:367, 2002.

[14] K. Sato, W. Schweika, P. H. Dederichs, and H. Katayama-Yoshida. Low-temperature ferromagnetism in (Ga, Mn)N: Ab initio calculations. Physical Review B, 70:201202, 2004.

[15] G. M. Dalpian and S. H. Wie. Electron-induced stabilization of ferromagnetism in $\mathrm{Ga}_{1-x} \mathrm{Gd}_{x}$ N. Physical Review B, 72:115201, 2005.

[16] T. Graf, M. Gjukic, M. S. Brandt, M. Stutzmann, and O. Ambacher. The $\mathrm{Mn}^{3+/ 2+}$ acceptor level in group III nitrides. Applied Physics Letters, 81:5159, 2002.

[17] W. Pacuski, D. Ferrand, P. Kossackia, S. Marcet, J. Cibert, J.A. Gaj, and A. Golnik. Excitonic Giant Zeeman Effect in Wide Gap Diluted Magnetic Semiconductors Based on ZnO and GaN. Acta Physica Polonica A, 110:303, 2006.

[18] M.-H. Ham, S. Yoon, Y. Park, L. Bian, M. Ramsteiner, and J.-M. Myoung. Electrical spin injection from room-temperature ferromagnetic $(\mathrm{Ga}, \mathrm{Mn}) \mathrm{N}$ in nitride-based spin-polarized light-emitting diodes. Journal of Physics: Condensed Matter, 18:7703, 2006.

[19] M. H. Kane, M. Strassburg, A. Asghar, N. Li, W. Fenwick, and I. T. Ferguson. Review of Recent Efforts on the Growth and Characterization of Nitride-based Diluted Magnetic Semiconductors. Proceedings of SPIE, 6121:61210L-1, 2006.

[20] M.E. Levinshtein, S.L. Rumyantsev, and M.S. Shur, editors. Properties of Advanced Semiconductor Materials, GaN, AlN, InN, BN, SiC, SiGe. John Wiley \& Sons, Inc., 2001.

[21] Ioffe Institute. Nsm archive. http://www.ioffe.rssi.ru/SVA/NSM/Semicond/ GaN/index.html, 2007.

[22] W. Martiensson and H. Warlimont, editors. Springer Handbook of Condensed Matter and Materials Data. Springer, 2005.

[23] S. Nakamura. Present performance of InGaN-based blue/green/yellow LEDs. volume 3002, pages 26-35. SPIE, 1997.

[24] F. Bernardini, V. Fiorentini, and D. Vanderbilt. Spontaneous polarization and piezoelectric constants of III-V nitrides. Physical Review B, 56:R1024, 1997. 
[25] S. Sugano, Y. Tanabe, and H. Kamimura. Multiplets of Transition-Metal Ions in Crystals, volume 33 of Pure and Applied Physics. Academic Press, 1970.

[26] B. Henderson and G.F. Imbusch. Optical Spectroscopy of Inorganic Solids. Oxford University Press, 1989.

[27] T. Graf, M. Gjukic, M. Hermann, M. S. Brandt, and M. Stutzmann. Spin resonance investigations of $\mathrm{Mn}^{2+}$ in wurtzite GaN and AlN films. Physical Review B, $67: 165215,2003$.

[28] B. Han, R. Y. Korotkov, B. W. Wessels, and M. P. Ulmer. Optical properties of $\mathrm{Mn}^{4+}$ ions in GaN:Mn codoped with Mg acceptors. Applied Physics Letters, 84:5320, 2004 .

[29] T. Graf, M. Gjukic, M. Hermann, M. S. Brandt, M. Stutzmann, L. Görgens, J. B. Philipp, and O. Ambacher. Growth and characterization of GaN:Mn epitaxial films. Journal of Applied Physics, 93:9697, 2003.

[30] Y. Tanabe and S. Sugano. On the Absorption Spectra of Complex Ions I. Journal of the Physical Society of Japan, 9:753, 1954.

[31] Y. Tanabe and S. Sugano. On the Absorption Spectra of Complex Ions II. Journal of the Physical Society of Japan, 9:766, 1954.

[32] Y. Tanabe and S. Sugano. On the Absorption Spectra of Complex Ions III The Calculation of the Crystalline Field Strength. Journal of the Physical Society of Japan, 11:864, 1956.

[33] H.A. Jahn and E. Teller. Stability of Polyatomic Molecules in Degenerate Electronic States. I. Orbital Degeneracy. Proceedings of the Royal Society of London, 161(905):220, 1937.

[34] H.A. Jahn. Stability of Polyatomic Molecules in Degenerate Electronic States. II. Spin Degeneracy. Proceedings of the Royal Society of London, 164(916):117, 1938.

[35] A. Wolos, A. Wysmolek, M. Kaminska, A. Twardowski, M. Bockowski, I. Grzegory, S. Porowski, and M. Potemski. Neutral Mn acceptor in bulk GaN in high magnetic fields. Physical Review B, 70:245202, 2004.

[36] F.S. Ham. Dynamical Jahn-Teller Effect in Paramagnetic Resonance Spectra: Orbital Reduction Factors and Partial Quenching of Spin-Orbit Interaction. Physical Review, 138:A1727, 1965.

[37] S. Marcet, D. Ferrand, D. Halley, S. Kuroda, H. Mariette, E. Gheeraert, F.J. Teran, M.L Sadowski, R. M. Galera, and J. Cibert. Magneto-optical spectroscopy of (Ga,Mn)N epilayers. Physical Review B, 74:125201, 2006.

[38] R. Y. Korotkov, J. M. Gregie, and B. W. Wesselsa. Optical properties of the deep Mn acceptor in GaN:Mn. Applied Physics Letters, 80:1731, 2002. 
[39] R.R. Galazka. Semimagnetic semiconductors based on mercury manganese telluride (HgMnTe) and cadmium manganese telluride (CdMnTe). Institute of Physics Conference Series, 43:133, 1979.

[40] P. Kacman. Spin interactions in diluted magnetic semiconductors and magnetic semiconductor structures. Semiconductor Science and Technology, 16:R25, 2001.

[41] B. E. Larson, K. C. Hass, H. Ehrenreich, and A. E. Carlsson. Theory of exchange interactions and chemical trends in diluted magnetic semiconductors. Phys. Rev. B, 37(8):4137-4154, Mar 1988.

[42] B. E. Larson and H. Ehrenreich. Anisotropic superexchange and spin-resonance linewidth in diluted magnetic semiconductors. Phys. Rev. B, 39(3):1747-1759, Jan 1989.

[43] P.H. Dederichs. Magnetism goes Nano, page E4. Forschungszentrum Jülich GmbH, 2005.

[44] J.K. Furdyna. Diluted magnetic semiconductors. Journal of Applied Physics, 64:R29, 1988.

[45] C. Zener. Interaction between the d shells in the transition metals. Physical Review, 81:440, 1951.

[46] H. Ohno, A. Shen, F. Matsukura, A. Oiwa, A. Endo, S. Katsumoto, and Y. Iye. (Ga,Mn)As: A new diluted magnetic semiconductor based on GaAs. Applied Physics Letters, 69(3):363-365, 1996.

[47] P. Mahadevan and A. Zunger. First-principles investigation of the assumptons underlying model-Hamiltonian approaches to ferromagnetism of $3 \mathrm{~d}$ impurities in III-V semiconductors. Physical Review B, 69:115211, 2004.

[48] K. Sato, H. Katayama-Yoshida, and P. H. Dederichs. High Curie Temperature and Nano-Scale Spinodal Decomposition Phase in Dilute Magnetic Semiconductors. Japanese Journal of Applied Physics, 44:L948, 2005.

[49] T. Fukushima, K. Sato, H. Katayama-Yoshida, and P. H. Dederichs. Spinodal Decomposition under Layer by Layer Growth Condition and High Curie Temperature Quasi-One-Dimensional Nano-Structure in Dilute Magnetic Semiconductors. Japanese Journal of Applied Physics, 45:L416, 2006.

[50] A. Svane, N. E. Christensen, L. Petit, Z. Szotek, and W. M. Temmerman. Electronic structure of rare-earth impurities in GaAs and GaN. Physical Review B, 74:165204, 2006.

[51] S. Dhar, O. Brandt, M. Ramsteiner, V. F. Sapega, and K. H. Ploog. Colossal Magnetic Moment of Gd in GaN. Physical Review Letters, 94:037205, 2005. 
[52] S. Dhar, L. Perez, O. Brandt, A. Trampert, K. H. Ploog, J. Keller, and B. Beschoten. Gd-doped GaN: A very dilute ferromagnetic semiconductor with a Curie temperature above 300 K. Physical Review B, 72:245203, 2005.

[53] T.D. Moustakas. Gallium Nitride II, volume 57 of Semiconductors and Semimetals, pages 33-128. Academic Press, 1999.

[54] S.P. DenBaars and S. Keller. Gallium Nitride I, volume 50 of Semiconductors and Semimetals, pages 11-38. Academic Press, 1998.

[55] R.J. Molnar. Gallium Nitride II, volume 57 of Semiconductors and Semimetals, pages 1-32. Academic Press, 1999.

[56] M. Röver. GaMnN - MBE auf Si(111). Diplomarbeit, Universität Göttingen, 2005.

[57] D. Mai. Magnetische und strukturelle Charakterisierung von (Ga,Mn)N. Diplomarbeit, Universität Göttingen, 2005.

[58] C. Adelmann, J. Brault, D. Jalabert, P. Gentile, H. Mariette, G. Mula, and B. Daudin. Dynamically stable gallium surface coverages during plasma-assisted molecularbeam epitaxy of (0001) GaN. Journal of Applied Physics, 91:9638, 2002.

[59] T. Zywietz, J. Neugebauer, and M. Scheffler. Adatom diffusion at GaN (0001) and

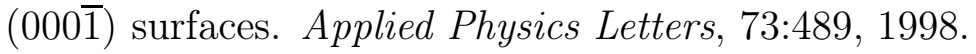

[60] J. E. Northrup, J. Neugebauer, R. M. Feenstra, and A. R. Smith. Structure of GaN(0001): The laterally contracted Ga bilayer model. Physical Review B, 61:9932, 2000 .

[61] G. Mula, C. Adelmann, S. Moehl, J. Oullier, and B. Daudin. Surfactant effect of gallium during molecular-beam epitaxy of GaN on AlN (0001). Physical Review B, 64:195406, 2001.

[62] C. Adelmann, J. Brault, E. Martinez-Guerrero, G. Mula, H. Mariette, L. S. Dang, and B. Daudin. Molecular-Beam Epitaxy of GaN: A Phase Diagram. physica status solidi (a), 188:575, 2001.

[63] C. Adelmann, J. Brault, G. Mula, and B. Daudin. Gallium adsorption on (0001) GaN surfaces. Physical Review B, 67:165419, 2003.

[64] B. Heying, R. Averbeck, L. F. Chen, E. Haus, H. Riechert, and J. S. Speck. Control of GaN surface morphologies using plasma-assisted molecular beam epitaxy. Journal of Applied Physics, 88:1855, 2000.

[65] J. Neugebauer. GaN surfaces and growth: The microscopic origin of surfactants and anti-surfactants. Vortrag, 2001. 
[66] M. Kocan, J. Malindretos, M. Roever, J. Zenneck, T. Niermann, D. Mai, M. Bertelli, M. Seibt, and A. Rizzi. Mn incorporation in GaN thin layers grown by molecularbeam epitaxy. Semiconductor Science and Technology, 21:1348, 2006.

[67] A.K. Viswanath. Processing and Properties of Compound Semiconductors, volume 73 of Semiconductors and Semimetals, pages 63-150. Academic Press, 2001.

[68] L. Liu and J. H. Edgar. Substrates for gallium nitride epitaxy. Material Science and Engineering R, 37:61, 2002.

[69] D.L. Dexter. A Theory of Sensitized Luminescence in Solids. Journal of Chemical Physics, 21:836, 1953.

[70] X.Z. Wang and B.W. Wessels. Investigation of Er-related centers in doped GaP. Materials Science Forum, 196:663, 1995.

[71] D. Volm, K. Oettinger, T. Streibl, D. Kovalev, M. Ben-Chorin, J. Diener, B. K. Meyer, J. Majewski, L. Eckey, A. Hoffmann, H. Amano, I. Akasaki, K. Hiramatsu, and T. Detchprohm. Exciton fine structure in undoped GaN epitaxial films. Phys. Rev. B, 53(24):16543-16550, Jun 1996.

[72] J.R. Haynes. Experimental Proof of the Existence of a New Electronic Complex in Silicon. Physical Review Letters, 4:361, 1960.

[73] M. A. Reshchikov and H. Morkoc. Luminescence properties of defects in GaN. Journal of Applied Physics, 97:061301, 2005.

[74] A. Shikanai, T. Azuhata, T. Sota, S. Chichibu, A. Kuramata, K. Horino, and S. Nakamura. Biaxial strain dependence of exciton resonance energies in wurtzite GaN. Journal of Applied Physics, 81:417, 1997.

[75] M. A. Reshchikov, D. Huang, F. Yun, L. He, H. Morkoc, D. C. Reynolds, S. S. Park, and K. Y. Lee. Photoluminescence of GaN grown by molecular-beam epitaxy on a freestanding GaN template. Applied Physics Letters, 79:3779, 2001.

[76] J.I. Pankove and J.A. Hutchby. Photoluminescence of ion-implanted GaN. Journal of Applied Physics, 47:5387, 1976.

[77] T. Ogino and M. Aoki. Mechanism of Yellow Luminescence in GaN. Japanese Journal of Applied Physics, 19:2395, 1980.

[78] D.M. Hofmann, D. Kovalev, G. Steude, B.K. Meyer, A. Hoffmann, L. Eckey, R. Heitz, T. Detchprom, H. Amano, and I. Akasaki. Properties of the yellow luminescence in undoped GaN epitaxial layers. Physical Review B, 52:16702, 1995.

[79] C.V. Raman and K.S. Krishnan. A New Type of Secondary Radiation. Nature, 121:501, 1928.

[80] P.Y. Yu and M. Cardona. Fundamentals of Semiconductors. Springer, 2001. 
[81] C. A. Arguello, D. L. Rousseau, and S. P. S. Porto. First-Order Raman Effect in Wurtzite-Type Crystals. Physical Review, 181:1351, 1969.

[82] H. Siegle, G. Kaczmarczyk, L. Filippidis, A. P. Litvinchuk, A. Hoffmann, and C. Thomsen. Zone-boundary phonons in hexagonal and cubic GaN. Physical Review $B, 55: 7000,1997$.

[83] T. Ruf, J. Serrano, M. Cardona, P. Pavone, M. Pabst, M. Krisch, M. D'Astuto, T. Suski, I. Grzegory, and M. Leszczynski. Phonon Dispersion Curves in WurtziteStructure GaN Determined by Inelastic X-Ray Scattering. Physical Review Letters, 86:906, 2001.

[84] K. Parlinski and Y. Kawazoe. Ab initio study of phonons in hexagonal GaN. Physical Review B, 60:15511, 1999.

[85] V. Yu. Davydov, N. S. Averkiev, I. N. Goncharuk, D. K. Nelson, I. P. Nikitina, A. S. Polkovnikov, A. N. Smirnov, M. A. Jacobson, and O. K. Semchinova. Raman and photoluminescence studies of biaxial strain in GaN epitaxial layers grown on 6H-SiC. Journal of Applied Physics, 82:5097, 1997.

[86] F. Demangeot, J. Frandon, M.A. Renucci, 0. Briot, B. Gil, and R.L. Aulombard. Raman Determination of phonon deformation potentials in $\alpha$-GaN. Solid State Communications, 100:207, 1996.

[87] C. Kisielowski, J. Krüger, S. Ruvimov, T. Suski, J.W. Ager, E. Jones, Z. LilientalWeber, M. Rubin, E.R. Weber, M.D. Bremser, and R.F. Davis. Strain-related phenomena in GaN thin films. Physical Review B, 54:17745, 1996.

[88] T. Kozawa, T. Kachi, H. Kano, H. Nagase, N. Koide, and K. Manabe. Thermal stress in GaN epitaxial layers grown on sapphire substrates. Journal of Applied Physics, 77:4389, 1995.

[89] J.-M. Wagner and F. Bechstedt. Phonon deformation potentials of alpha-GaN and -AlN: An ab initio calculation. Applied Physics Letters, 77:346, 2000.

[90] C. Kisielowski. Gallium Nitride II, volume 57 of Semiconductors and Semimetals, pages 275-318. Academic Press, 1999.

[91] T. Kozawa, T. Kachi, H. Kano, Y. Taga, M. Hashimoto, N. Koide, and K. Manabe. Raman scattering from LO phonon-plasmon coupled modes in gallium nitride. Journal of Applied Physics, 75:1098, 1994.

[92] P. Perlin, J. Camassel, W. Knap, T. Taliercio, J. C. Chervin, T. Suski, I. Grzegory, and S. Porowski. Investigation of longitudinal-optical phonon-plasmon coupled modes in highly conducting bulk GaN. Applied Physics Letters, 67:2524, 1995.

[93] P. Thurian, G. Kaczmarczyk, H. Siegle, R. Heitz, A. Hoffmann, I. Broser, B.K. Meyer, R. Hoffbauer, and U. Scherz. Local vibrational modes of 3d elements in wurtzite type ZnO and GaN crystals. Materials Science Forum, 196:1571, 1995. 
[94] Hiroshi Harima. Properties of GaN and related compounds studied by means of Raman scattering. Journal of Physics: Condensed Matter, 14:R967, 2002.

[95] M. Thompson. Rump. http://www.genplot.com/index.htm, 2002.

[96] J.F. Ziegler, J.P. Biersack, and U. Littmark. The stopping and range of ions in solids. Pergamon Press, New York, 1999.

[97] JCPDS-ICDD. Pcpdfwin. Software, 1995.

[98] P. l'Heritier, D. Fruchart, R. Madar, and R. Fruchart. Instabilités électroniques dans les composés du manganese de type perovskite métallique. Relation avec les changements de valence dans les composés de terres rares. Material Research Bulletin, 14:1089, 1979 .

[99] J.P. Bouchaud. Propriétés magnétiques et structurales des carbonitrures de manganèse et des perowskites $\mathrm{Mn}_{3} \mathrm{GaC}$ et $\mathrm{Mn}_{3} \mathrm{GaN}$. Annales de Chimie, 3:81, 1968 .

[100] K. H. Kim, K. J. Lee, D. J. Kim, H. J. Kim, Y. E. Ihm, D. Djayaprawira, M. Takahashi, C. S. Kim, C. G. Kim, and S. H. Yoo. Magnetotransport of p-type GaMnN assisted by highly conductive precipitates. Applied Physics Letters, 82:1775, 2003.

[101] K. H. Kim, K. J. Lee, D. J. Kim, H. J. Kim, Y. E. Ihm, C. G. Kim, S. H. Yoo, and C. S. Kim. Enhanced carrier-mediated ferromagnetism in GaMnN by codoping of Mg. Applied Physics Letters, 82:4755, 2003.

[102] S. Kuroda, E. Bellet-Amalric, R. Giraud, S. Marcet, J. Cibert, and H. Mariette. Strong influence of $\mathrm{Ga} / \mathrm{N}$ flux ratio on $\mathrm{Mn}$ incorporation into $\mathrm{Ga}_{1-x} \mathrm{Mn}_{x} \mathrm{~N}$ epilayers grown by plasma-assisted molecular beam epitaxy. Applied Physics Letters, 83:4580, 2003.

[103] S. Kuroda, E. Bellet-Amalric, X. Biquard, J. Cibert, R. Giraud, S. Marcet, and H. Mariette. Optimization of the growth of $\mathrm{Ga}_{1-x} \mathrm{Mn}_{x} \mathrm{~N}$ epilayers using plasmaassisted MBE. physica status solidi (b), 240:443, 2003.

[104] K. J. Lee, F. C. Yu, J. A. Kim, D. J. Kim, B. G. Kim, Y. H. Kang, H. J. Kim, and Y. E. Ihm. Behavior of precipitates in GaMnN. physica status solidi (b), 241:1525, 2004.

[105] K. H. Kim, K. J. Lee, H. S. Kang, F. C. Yu, J. A. Kim, D. J. Kim, K. H. Baik, S. H. Yoo, C. G. Kim, Y. S. Kim, C. S. Kim, H. J. Kim, and Y. E. Ihm. Molecular beam epitaxial growth of GaN and GaMnN using a single precursor. physica status solidi (b), 241:1458, 2004.

[106] R. Giraud, S. Kuroda, S. Marcet, X. Biquard E. Bellet-Amalric, B. Barbara, D. Fruchart, D. Ferrand, J. Cibert, and H. Mariette. Ferromagnetic $\mathrm{Ga}_{1-x} \mathrm{Mn}_{x} \mathrm{~N}$ epilayers vs. Antiferromagnetic $\mathrm{GaMn}_{3} \mathrm{~N}$ clusters. Europhysics Letters, 65:553, 2004. 
[107] B. Ha, H. C. Kim, S.-G. Kang, Y. H. Kim, J. Y. Lee, C. Y. Park, and C. J. Lee. Synthesis and Characterization of Heterostructured $\mathrm{Mn}_{3} \mathrm{GaN}_{0.5} / \mathrm{GaN}$ Nanowires. Chemical Materials, 17:5398, 2005.

[108] H. Nakayama, H. Mashita, E. Kulatov, R. Funahashi, and H. Ohta. MBE growth and properties of room-temperature $(\mathrm{Ga}, \mathrm{Mn}) \mathrm{N}-\mathrm{Mn}_{4} \mathrm{~N}$ granular magnetic semiconductors. Journal of Magnetism and Magnetic Materials, 258:323, 2003.

[109] M. Zajac, R. Doradzinski, J. Gosk, J. Szczytko, M. Lefeld-Sosnowska, M. Kaminska, A. Zwardowski, and E. Grzanka. Magnetic and optical properties of GaMnN magnetic semiconductor. Applied Physics Letters, 78:1276, 2001.

[110] Y. Cui and L. Li. Suppression of phase segregation during melecular-beam epitaxial growth of GaMnN using nitrogen-hydrogen plasma. Applied Physics Letters, 80:4139, 2002.

[111] D. Fruchart and E.F. Bertaut. Magnetic Studies of the Metallic Perovskite-Type Compounds of Manganese. Journal of the Physical Society of Japan, 44:781, 1978.

[112] M. S. Miao, A. Herwadkar, and W. R. L. Lambrecht. Electronic structure and magnetic properties of $\mathrm{Mn}_{3} \mathrm{GaN}$ precipitates in $\mathrm{Ga}_{1-x} \mathrm{Mn}_{x} \mathrm{~N}$. Physical Review B, 72:033204, 2005.

[113] T. Niermann. Mikrostrukturelle Untersuchungen an Mangan-dotiertem Galliumnitrid mittels fortgeschrittener Methoden der hochauflösenden und analytischen Transmissionselektronenmikroskopie. Dissertation, Universität Göttingen, 2006.

[114] H. Harima. Raman studies on spintronics materials based on wide bandgap semiconductors. Journal of Physics: Condensed Matter, 16:S5653, 2004.

[115] W. Limmer, W. Ritter, R. Sauer, B. Mensching, C. Liu, and B. Rauschenbach. Raman scattering in ion-implanted GaN. Applied Physics Letters, 27:2589, 1998.

[116] M.H. Kane, M. Strassburg, A. Asghar, W.E. Fenwick, J. Senawiratne, Q. Song, C.J. Summers, Z.J. Zhang, N. Dietz, and I.T. Ferguson. Alloying, co-doping, and annealing effects on the magnetic and optical properties of MOCVD-grown $\mathrm{Ga}_{1-x} \mathrm{Mn}_{x} \mathrm{~N}$. Material Science and Engineering B, 126:230, 2006.

[117] W. Gebicki, J. Strzeszewski, G. Kamler, T. Szyszko, and S. Podsiadlo. Raman scattering study of $\mathrm{Ga}_{1-x} \mathrm{Mn}_{x} \mathrm{~N}$ crystals. Applied Physics Letters, 76:3870, 2000.

[118] M. Asghar, I. Hussain, F. Saleemi, E. Bustarret, J. Cibert, S. Kuroda, S. Marcet, H. Mariette, and A.S. Bhatti. Properties of $\mathrm{Ga}_{1-x} \mathrm{Mn}_{x} \mathrm{~N}$ epilayers grown by plasmaassisted molecular beam epitaxy using Raman spectroscopy. Material Science and Engineering B, 133:102, 2006.

[119] J. Neugebauer and C.G. van de Walle. Atomic geometry and electronic structure of native defects in GaN. Physical Review B, 50:8067, 1994. 
[120] C. G.van de Walle and J. Neugebauer. First-principles calculations for defects and impurities: Applications to III-nitrides. Journal of Applied Physics, 95:3851, 2004.

[121] J. Zenneck, T. Niermann, D. Mai, M. Roever, M. Kocan, J. Malindretos, M. Seibt, A. Rizzi, N. Kaluza, and H. Hardtdegen. Intra-atomic photoluminescence at $1.41 \mathrm{eV}$ of substitutional Mn in GaMnN of high optical quality. Journal of Applied Physics, 101:063504, 2007.

[122] Y. L. Soo, G. Kioseoglou, S. Kim, S. Huang, Y. H. Kao, S. Kuwabara, S. Owa, T. Kondo, and H. Munekata. Local structure and chemical valency of Mn impurities in wide-band-gap III-V magnetic alloy semiconductors $\mathrm{Ga}_{1-x} \mathrm{Mn}_{x} \mathrm{~N}$. Applied Physics Letters, 79:3926, 2001.

[123] G. Thaler, R. Frazier, B. Gila, J. Stapleton M. Davidson, C.R. Abernathy, and C. Segre. Effect of Mn concentration on the structural, optical and magnetic properties of GaMnN. Applied Physics Letters, 84:1314, 2004.

[124] J. M. Baik, S. U. Kim, Y. M. Koo, T. W. Kang, and J.-L. Lee. Evidence of Mn Occupation of Ga Site in Ferromagnetic (Ga,Mn)N Semiconductor Observed by EXAFS. Electrochemical and Solid-State Letters, 7:G313, 2004.

[125] L.A. Carreno and A. Bensaoula. Combination of time of flight direct recoiled spectroscopy and ion scattering trajectory simulations of (Ga,Mn)N growth by chemical beam epitaxy. Applied Physics Letters, 86:021901, 2005.

[126] B. Monemar, J.P. Bergman, and I.A. Buyanova. GaN and Related Materials, page 85. Gordon and Breach, 1997.

[127] K. Kornitzer, T. Ebner, M. Grehl, K. Thonke, R. Sauer, C. Kirchner, V. Schwegler, M. Kamp, M. Leszczynski, I. Grzegory, and S. Porowski. High-Resolution Photoluminescence and Reflectance Spectra of Homoepitaxial GaN Layers. physica status solidi (b), 216:5, 1999 .

[128] K. Kornitzer, T. Ebner, M. Grehl, K. Thonke, R. Sauer, C. Kirchner, V. Schwegler, M. Kamp, M. Leszczynski, I. Grzegory, and S. Porowski. Photoluminescence and reflectance spectroscopy of excitonic transitions in high-quality homoepitaxial GaN films. Physical Review B, 60:1471, 1999.

[129] A. Wysmolek, M. Potemski, R. Stepniewski, J. Lusakowski, K. Pakula, J. M. Baranowski, G. Martinez, P. Wyder, I. Grzegory, and S. Porowski. Polarised Magnetoluminescence of Excitons in Homoepitaxial GaN Layers. physica status solidi (b), 216:11, 1999 .

[130] F. Calle, F. J. Sanchez, J. M. G. Tijero, M. A. Sanchez-Garcia, E. Calleja, and R. Beresford. Exciton and donor-acceptor recombination in undoped GaN on Si(111). Semiconductor Science and Technology, 12:1396, 1997. 
[131] M. Godlewski, H. Przybylinska, R. Bozek, E. M. Goldys, J. P. Bergman, B. Monemar, I. Grzegory, and S. Porowski. Compensation mechanisms in magnesium doped GaN. physica status solidi (a), 201:216, 2004.

[132] J. Baur, K. Maier, M. Kunzer, U. Kaufmann, J. Schneider, H. Amano, I. Akasaki, T. Detchprohm, and K. Hiramatsu. Infrared luminescence of residual iron deep level acceptors in gallium nitride (GaN) epitaxial layers. Applied Physics Letters, 64:857, 1994.

[133] J. Zenneck. Kathodolumineszenz-Untersuchungen an implantiertem AluminiumNitrid. Diplomarbeit, Universität Göttingen, 2003.

[134] U. Vetter. Lanthanide Doped Wide Band Gap Semiconductors: Intra-4f Luminescence and Lattice Location Studies. Dissertation, Universität Göttingen, 2003.

[135] I. Vurgaftman, J. R. Meyer, and L. R. Ram-Mohan. Band parameters for III-V compound semiconductors and their alloys. Journal of Applied Physics, 89:5815, 2001.

[136] I. Vurgaftman and J. R. Meyer. Band parameters for nitrogen-containing semiconductors. Journal of Applied Physics, 94:3675, 2003.

[137] R.Y. Korotkov, J.M. Gregie, and B.W. Wessels. Mn-related absorption and PL bands in GaN grown by metal organic vapor phase epitaxy. Physica B, 308:30, 2001.

[138] A. Wolos, M. Palczewska, M. Zajac, J. Gosk, M. Kaminska, A. Twardowski, M. Bockowski, I. Grzegory, and S. Porowski. Optical and magnetic properties of Mn in bulk GaN. Physical Review B, 69:115210, 2004.

[139] S. Marcet, D. Ferrand, S. Kuroda, E. Gheeraert, R. M. Galera, J. Cibert, and H. Mariette. Magneto-optical spectroscopy of the wide band gap diluted magnetic semiconductor GaMnN. Material Science and Engineering B, 126:240, 2006.

[140] M. Strassburg, M.H. Kane, A. Asghar, Q. Song, Z.J. Zhang, J. Senawiratne, M. Alevli, N. Dietz, C.J. Summers, and I.T. Ferguson. The Fermi level dependence of the optical and magnetic properties of $\mathrm{Ga}_{1-x} \mathrm{Mn}_{x} \mathrm{~N}$ grown by metal-organic chemical vapour deposition. Journal of Physics: Condensed Matter, 18:2615, 2006.

[141] T. Graf, M. Gjukic, L. Görgens, O. Ambacher, M. S. Brandt, and M. Stutzmann. Charge Transfer at the Mn Acceptor Level in GaN. Journal of Superconductivity, 16:83, 2003.

[142] A. Y. Polyakov, N. B. Smirnov, A. V. Govorkov, N. Y. Pashkova, J. Kim, F. Ren, M. E. Overberg, G. T. Thaler, C. R. Abernathy, S. J. Pearton, and R. G. Wilson. Electrical and optical properties of GaN films implanted with Mn and Co. Journal of Applied Physics, 92:3130, 2002. 
[143] E. Kulatov, H. Nakayama, H. Mariette, H. Ohta, and Y. A. Uspenskii. Electronic structure, magnetic ordering, and optical properties of GaN and GaAs doped with Mn. Physical Review B, 66:045203, 2002.

[144] Z. S. Popovic, S. Satpathy, and W.C. Mitchel. Electronic structure of the substitutional versus interstitial manganese in GaN. Physical Review B, 70:161308, 2004.

[145] B. Gil. Gallium Nitride II, volume 57 of Semiconductors and Semimetals, pages 209-274. Academic Press, 1999.

[146] S. Marcet. Étude magnéto-optique de $(G a, M n) N$ et $(Z n, C r) T e$. Dissertation, Université Joseph Fourier, 2005.

[147] R. Stepniewski, M. Potemski, A. Wysmolek, K. Pakula, J.M. Baranowski, J. Lusakowski, I. Grzegory, S. Porowski, G. Martinez, and P. Wyder. Symmetry of excitons in GaN. Physical Review B, 60:4438, 1999.

[148] P. A. Shields, R. J. Nicholas, B. Beaumont, , and P. Gibart. Magneto-Reflectivity of Gallium Nitride Epilayers. physica status solidi (b), 216:17, 1999.

[149] A. V. Rodina, M. Dietrich, A. Göldner, L. Eckey, Al. L. Efros, M. Rosen, A. Hoffmann, and B. K. Meyer. Exciton Energy Structure in Wurtzite GaN. physica status solidi (b), 216:21, 1999.

[150] W. Pacuski, D. Ferrand, J. Cibert, J. A. Gaj, A. Golnik, P. Kossacki, S. Marcet, E. Sarigiannidou, and H. Mariette. Excitonic giant Zeeman effect in GaN:Mn ${ }^{3+}$. arXiv / cond-mat, 0:0703041, 2007.

[151] M. Kaminska. persönliche Mitteilung.

[152] D. Ferrand. persönliche Mitteilung.

[153] M. Hashimoto, S. Emura, H. Tanaka, T. Honma, N. Umesaki, S. Hasegawa, and H. Asahi. Local crystal structure and local electronic structure around Cr in lowtemperature-grown GaCrN layers. Journal of Applied Physics, 100:103907, 2006.

[154] U. Gerstmann, A. T. Blumenau, and H. Overhof. Transition metal defects in groupIII nitrides: An ab initio calculation of hyperfine interactions and optical transitions. Physical Review B, 63:075204, 2001.

[155] Y.S. Cho, N. Kaluza, V. Guzenko, Th. Schäpers, H. Hardtdegen, H.-P. Bochem, U. Breuer, M. R. Ghadimi, M. Fecioru-Morariu, B. Beschoten, and H. Lüth. The growth of Cr-doped GaN by MOVPE towards spintronic applications. physica status solidi (a), 204:72, 2007.

[156] I.T. Yoon, T.W. Kang, M.C. Jeong, M.H. Ham, and J.M. Myoung. Observation of a maganese acceptor level in ferromagnetic GaMnN layers. Applied Physics Letters, 85:4878, 2004. 
[157] M. H. Kane, A. Asghar, C. R. Vestal, M. Strassburg, J. Senawiratne, Z. J. Zhang, N. Dietz, C. J. Summers, and I. T. Ferguson. Magnetic and optical properties of $\mathrm{Ga}_{1-x} \mathrm{Mn}_{x} \mathrm{~N}$ grown by metalorganic chemical vapour deposition. Semiconductor Science and Technology, 20:L5, 2005.

[158] M.H. Kane, S. Gupta, W.E. Fenwick, N. Li, E.-H. Park, M. Strassburg, and I.T. Ferguson. Comparative study of $\mathrm{Mn}$ and Fe incorporation into GaN by metalorganic chemical vapor deposition. physica status solidi (a), 204:61, 2007.

[159] A. Y. Polyakov, N. B. Smirnov, A. V. Govorkov, N. V. Pashkova, A. A. Shlensky, S. J. Pearton, M. E. Overberg, C. R. Abernathy, J. M. Zavada, and R. G. Wilson. Electrical and optical properties of $\mathrm{Cr}$ and Fe implanted n-GaN. Journal of Applied Physics, 93:5388, 2003.

[160] Y.-K. Zhou, M. Hashimoto, M. Kanamura, and H. Asahi. Room temperature ferromagnetism in III-V-based diluted magnetic semicoductor $\mathrm{GaCrN}$ grown by ECR molecular-beam epitaxy. Journal of Superconductivity, 16:37, 2003.

[161] Y.K. Zhou, M.S. Kim, X.J. Li, S. Kimura, A. Kaneta, Y. Kawakami, Sg. Fujita, S. Emura, S. Hasegawa, and H. Asahi. Optical properties of GaN-based magnetic semiconductors. Journal of Physics: Condensed Matter, 16:S5743, 2004.

[162] H. Asahi, Y.K. Zhou, M. Hashimoto, M.S. Kim, X.J. Li, S. Emura, and S. Hasegawa. GaN-based magnetic semiconductors for nanospintronics. Journal of Physics: Condensed Matter, 16:S5555, 2004.

[163] A.Y. Polyakov, N.B. Smirnov, A.V. Govorkov, G.T. Thaler, R.M. Frazier, C.R. Abernathy, and S.J. Pearton. Electrical and optical properties of GaCrN films grown by molecular beam epitaxy. Journal of Vacuum Science and Technology B, 23:1, 2005.

[164] S. Shanthi, M. Hashimoto, Y. K. Zhou, S. Kimura, M. S. Kim, S. Emura, N. Hasuike, H. Harima, S. Hasegawa, M. Ishimaru, Y. Hirotsu, and H. Asahi. Polymorphism in the ferromagnetic GaCrN-diluted magnetic semiconductor: Luminescence and structural investigations. Journal of Applied Physics, 98:013526, 2005.

[165] S. Subashchandran, S. Kimura, M. S. Kim, S. Kobayashi, Y. K. Zhou, S. Hasegawa, and H. Asahi. Nature of Deep-Level Defects in GaCrN Diluted Magnetic Semiconductor. Japanese Journal of Applied Physics, 45:3522, 2006.

[166] M. A. Reshchikov, F. Shahedipour, R. Y. Korotkov, B. W. Wessels, and M. P. Ulmer. Photoluminescence band near $2.9 \mathrm{eV}$ in undoped GaN epitaxial layers. Journal of Applied Physics, 87:3351, 2000.

[167] C. Zuo and P.W. Jagodzinski. R-line Luminescence from Trace Amounts of $\mathrm{Cr}^{3+}$ in Aluminum Oxide and Its Dependence on Sample Hydration. Appl. Spect., 56(8):1055 - 1058, 2002. 
[168] T. Maiman. Stimulated optical radiation in ruby. Nature, 187(4736):493 - 694, 1960.

[169] V. E. Kudryashov, S. S. Mamakin, and A. E. Yunovich. Influence of a sapphire substrate on the emission spectra of gallium nitride lightemitting diodes. Technical Physics Letters, 25:536, 1999.

[170] J. Baur, U. Kaufmann, M. Kunzer, J. Schneider, H. Amano, I. Akasaki, T. Detchprohm, and K. Hiramatsu. Photoluminescence of residual transition metal impurities in GaN. Applied Physics Letters, 67:1140, 1995.

[171] R. Heitz, P. Thurian, I. Loa, L. Eckey, A. Hoffmann, I. Broser, K. Pressel, B.K. Meyer, and E.N. Mokhov. Identification of the $1.19 \mathrm{eV}$ luminescence in hexagonal GaN. Physical Review B, 52:16508, 1995.

[172] R. Heitz, P. Maxim, L. Eckey, P. Thurian, A. Hoffmann, I. Broser, K. Pressel, and B.K. Meyer. Excited states of $\mathrm{Fe}^{3+}$ in GaN. Physical Review B, 55:4382, 1997.

[173] C. Elschenbroich. Organometallchemie. Teubner, 2005.

[174] E. Dörksen. persönliche Mitteilung.

[175] M. Hashimoto, S. Emura, R. Asano, H. Tanaka, N. Teraguchi, A. Suzuki, Y. Nanishi, T. Honma, N. Umesaki, and H. Asahi. Local structure of rare-earth-doped diluted magnetic semiconductor GaGdN. physica status solidi (c), 0:2650, 2003.

[176] U. Vetter, J. Zenneck, and H. Hofsäss. Intense ultraviolet cathodoluminescence at 318 nm from $\mathrm{Gd}^{3+}$-doped AlN. Applied Physics Letters, 83:2145, 2003.

[177] Z. Lipinska, M. Pawlowski, H. Zolnierowicz, A. Wysmolek, M. Palczewska, M. Kaminska, A. Twardowski, M. Bockowski, and I. Grzegory. Photoluminescence and electron paramagnetic resonance studies of bulk GaN doped with gadolinium. Acta Physica Polonica A, 110:243, 2006.

[178] S. W. Choi, S. Emura, S. Kimura, M. S. Kim, Y. K. Zhou, N. Teraguchi, A. Suzuki, A. Yanase, and H. Asahi. Emission spectra from AlN and GaN doped with rare earth elements. Journal of Alloys and Compounds, 408:717, 2006.

[179] Y.-K. Kuo, W.-W. Lin, and J. Lin. Band-Gap Bowing Parameter of the $\operatorname{In}_{x} \operatorname{Ga}_{1-x} \mathrm{~N}$ Derived From Theoretical Simulation. Japanese Journal of Applied Physics, 40:3157, 2001.

[180] N. Teraguchi, A. Suzuki, Y. Nanishi, Y.-K. Zhou, M. Hashimoto, and H. Asahi. Room-temperature observation of ferromagnetism in diluted magnetic semiconductor GaGdN grown by RF-molecular beam epitaxy. Solid State Communications, 122:651, 2002.

[181] F. Leuenberger. Elemental resolved magnetism of Gadoliniumnitride layers and GdN/Fe multilayers. Dissertation, Universität Göttingen, 2004. 
[182] C. M. Aerts, P. Strange, M. Horne, W. M. Temmerman, Z. Szotek, and A. Svane. Half-metallic to insulating behavior of rare-earth nitrides. Physical Review B, 69:045115, 2004. 


\section{Danke}

- an Prof. Dr. Angela Rizzi für die Möglichkeit zur Promotion auf dem interessanten und nach wie vor nur teilweise erforschten Terrain der verdünnten magnetischen Halbleiter und für ausgezeichnete Betreuung und stete Motivation.

- an Prof. Dr. Hans Hofsäss für die freundliche Übernahme des Koreferats sowie Ihm und der gesamten II. Physik dafür, dass ich mich dort auch in den Laboren hin und wieder rumtreiben durfte.

- an Dr. Jörg Malindretos für die vielen kleinen und großen Probleme, mit denen man Ihn immer belästigen durfte und die er praktisch immer lösen konnte. Der einzige Korrekturleser, der sich durch den kompletten Theorieteil gekämpft hat!

- an Prof. Dr. Rainer G. Ulbrich und Dr. Matthias Erdmann für die Unterstützung im Labor und bei PL allgemein, sowie bei der Betreuung des Halbleiterpraktikums.

- an Du Mai, Martin Röver und Dr. Martin Kočan für die GaMnN- und GaGdNProben. Ohne Euch hätte ich nichts zu messen gehabt! Und nur halb so viele Parties. Außerdem haben die ersten beiden brav große Teile der Arbeit Korrektur gelesen.

- an Prof. Dr. Hilde Hardtdegen, Dr. Nicoleta Kaluza und Yong Suk Cho (a.k.a. Denny) für die GaCrN-Proben, sowie die vielen hilfreichen Diskussionen über $\mathrm{GaCrN}$ und MOCVD.

- an Marco Bertelli, Mitstreiter und Zimmerkollege seit Stunde 1, der wie ich die Metamorphose vom Lichtenberg-Jünger zum Wiesel durchlaufen hat, für all die gute Zeit und vor allem die gute Pasta.

- an Dr. Tore Niermann und die TEM-Gruppe für die schönen Bilder, sowie die Ergebnisse, die sich perfekt mit meinen ergänzt haben.

- an Daniel Broxtermann, Christian Denker, Amilcar Bedoya-Pinto, Sönke Hüls und den ganzen Schwarm an neuen Diplomanden und oben bereits genannten für die gute Atmosphäre in der Arbeitsgruppe, die sich auch in vielen abendlichen Aktionen geäußert hat...

- an die Tunnelgruppe, wo ich immer um Helium gebettelt habe und nie zurückgeschickt wurde. 
- an die Werkstätten und hier besonders Klaus Langohr, der auch für meine alten 386er noch Ersatzteile auf Lager hatte.

- an die immer gut gelaunten und freundlichen Sekretärinnen: Martina Ackermann, Natascha Schneider (damals Feuker) und Antje Spliethoff-Laiser. Nur dank Ihnen habe ich keine Termine für die Reisekostenabrechnung verpasst!

- à Wojciech Pacuski et Dr. David Ferrand de l'université de Grenoble pour la collaboration et les manipes magnéto-optiques.

- an Dr. Peter Wilbrandt für die SIMS-Profile, selbst wenn es manchmal schlechte Nachrichten für mich waren.

- an Dr. Yuansu Luo und das I. Physikalische Institut, Dieter Plischke und das Institut für Materialphysik sowie Prof. Dr. Peter Schaaf und das II. Physikalische Institut für die Einweisungen und die Möglichkeiten für XRD-Messungen.

- an Dr. Helge Bay für die RBS-Messungen in Jülich und an Dr. Michael Uhrmacher und Dr. Carsten Ronning für die Implantationen am II. Physikalischen Institut.

- a Dr. Eva Monroy por las interesantes discusiones sobre nitruros y luminiscencia, las cuales me fueron de gran ayuda, especialmente en la primera etapa de mi proyecto.

- an das Land Niedersachsen für die Gewährung des Georg-Christoph-LichtenbergStipendiums mit der entsprechenden finanziellen Unterstützung.

- an das Virtuelle Institut für SpinELektronik (VISEL) für die finanzielle Unterstützung und die fruchtbaren Diskussionen, Ideen und Kollaborationen, die sich aus dieser Zusammenarbeit zwischen Aachen, Jülich und Göttingen ergeben haben.

- an Freunde und Familie. Ja, es gibt ein Leben außerhalb der Physik. Dafür danke ich Euch allen und ganz besonders Elina, ohne die dieses Leben nur halb so schön gewesen wäre. 


\section{Lebenslauf}

$\begin{array}{ll}\text { Name } & \text { Jan Zenneck } \\ \text { Geburtstag } & 13.07 .1978 \\ \text { Geburtsort } & \text { Marburg } \\ \text { Staatsangehörigkeit } & \text { Deutsch }\end{array}$

\section{Ausbildung}

$1984-1988$

$1988-1991$

1991-1997

$1997-1998$

$1998-2000$

$2000-2001$

2001-2003

seit 2003
Theodor-Heuss Grund- und Hauptschule Sinsheim

Wilhemi-Gymnasium Sinsheim

Ehrenbürg-Gymnasium Forchheim, Abitur 1997

Zivildienst bei der Bahnhofsmission Erlangen

Friedrich-Alexander-Universität Erlangen-Nürnberg, Physik Vordiplom 2000

Université des Sciences et Technologies de Lille Georg-August-Universität Göttingen, Physik Diplom 2003

Doktorand am IV. Physikalischen Institut, Georg-AugustUniversität Göttingen 